\title{
A COMPARISON OF WAVELET AND SIMPLICITY-BASED HEART SOUND AND MURMUR SEGMENTATION METHODS
}

\author{
A Thesis \\ presented to \\ the Faculty of California Polytechnic State University, \\ San Luis Obispo
}

\author{
In Partial Fulfillment \\ of the Requirements for the Degree \\ Master of Science in Electrical Engineering
}

by

Joshua David Korven

September 2016 
(C) 2016

Joshua David Korven

ALL RIGHTS RESERVED 
COMMITTEE MEMBERSHIP

TITLE: $\quad$ A Comparison of Wavelet and Simplicity-Based Heart Sound and Murmur Segmentation Methods

AUTHOR: Joshua David Korven

DATE SUBMITTED: $\quad$ September 2016

COMMITTEE CHAIR: Wayne Pilkington, Ph.D.

Associate Professor of Electrical Engineering

COMMITTEE MEMBER: Jane Zhang, Ph.D.

Professor of Electrical Engineering

COMMITTEE MEMBER: John A. Saghri, Ph.D.

Professor of Electrical Engineering 


\begin{abstract}
A Comparison of Wavelet and Simplicity-Based Heart Sound and Murmur Segmentation Methods

Joshua David Korven
\end{abstract}

Stethoscopes are the most commonly used medical devices for diagnosing heart conditions because they are inexpensive, noninvasive, and light enough to be carried around by a clinician. Auscultation with a stethoscope requires considerable skill and experience, but the introduction of digital stethoscopes allows for the automation of this task. Auscultation waveform segmentation, which is the process of determining the boundaries of heart sound and murmur segments, is the primary challenge in automating the diagnosis of various heart conditions. The purpose of this thesis is to improve the accuracy and efficiency of established techniques for detecting, segmenting, and classifying heart sounds and murmurs in digitized phonocardiogram audio files. Two separate segmentation techniques based on the discrete wavelet transform (DWT) and the simplicity transform are integrated into a MATLAB software system that is capable of automatically detecting and classifying sound segments.

The performance of the two segmentation methods for recognizing normal heart sounds and several different heart murmurs is compared by quantifying the results with clinical and technical metrics. The two clinical metrics are the false negative detection rate (FNDR) and the false positive detection rate (FPDR), which count heart cycles rather than sound segments. The wavelet and simplicity methods have a $4 \%$ and $9 \%$ respective FNDR, so it is unlikely that either method would not detect a heart condition. However, the $22 \%$ and $0 \%$ respective FPDR signifies that the wavelet method is likely to detect false heart conditions, while the simplicity method is not. The two technical metrics are the true 
murmur detection rate (TMDR) and the false murmur detection rate (FMDR), which count sound segments rather than heart cycles. Both methods are equally likely to detect true murmurs given their $83 \%$ TMDR. However, the $13 \%$ and $0 \%$ respective FMDR implies that the wavelet method is susceptible to detecting false murmurs, while the simplicity method is not. Simplicity-based segmentation, therefore, demonstrates superior performance to wavelet-based segmentation, as both are equally likely to detect true murmurs, but only the simplicity method has no chance of detecting false murmurs.

Keywords: Phonocardiogram, PCG, Stethoscope, Segmentation, Discrete Wavelet Transform, DWT, Simplicity Transform, Beamforming 


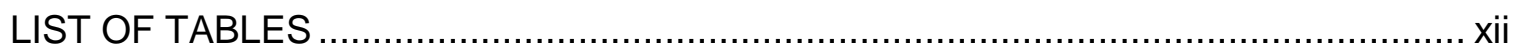

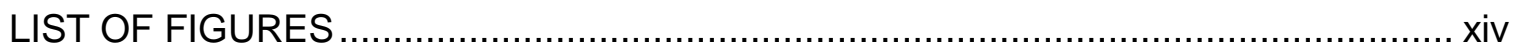

\section{CHAPTER}

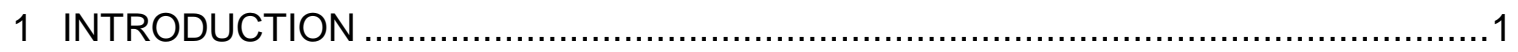

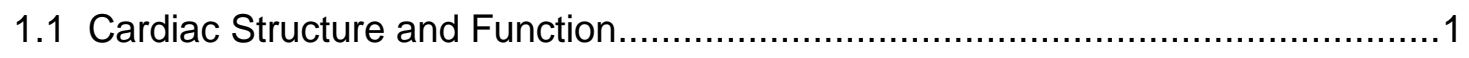

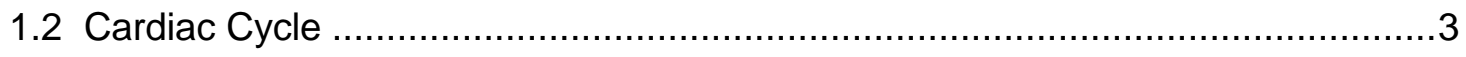

1.3 Auscultation, Heart Sounds, \& Murmurs …………..................................

1.4 Heart Sound and Murmur Segmentation Goals ...........................................

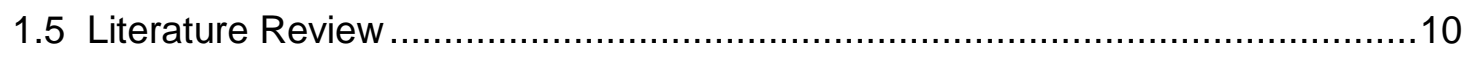

1.6 Proposed Modifications to the Established Methods …...................................12

2 VIABILITY OF USING A STETHOSCOPE ARRAY FOR IMPROVED HEART

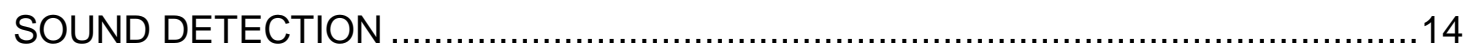

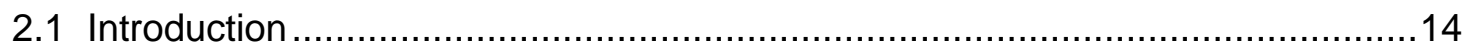

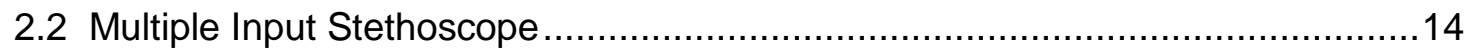

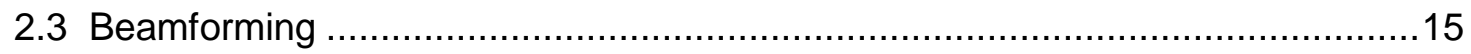

2.4 Acoustic Aperture

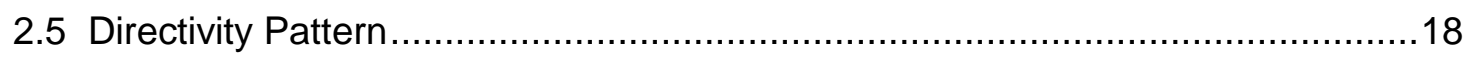

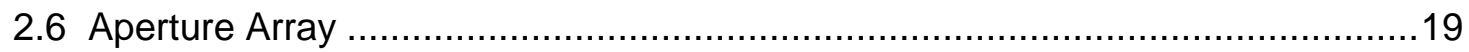

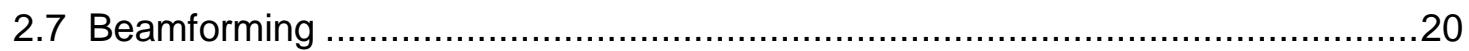

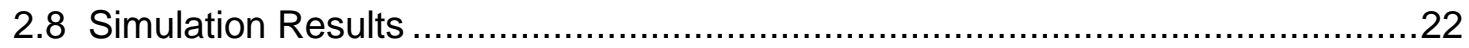

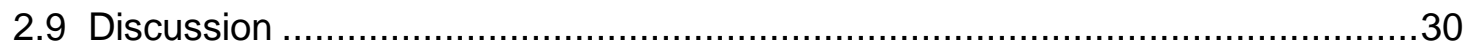

3 SEGMENTATION ALGORITHMS AND CONCEPTS .............................................

3.1 Frequency Domain Filtering …………………....................................... 


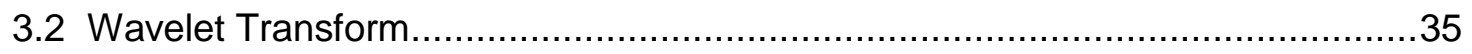

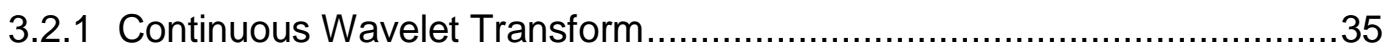

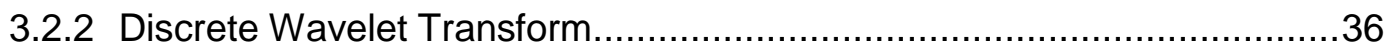

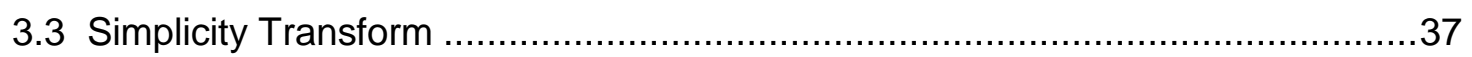

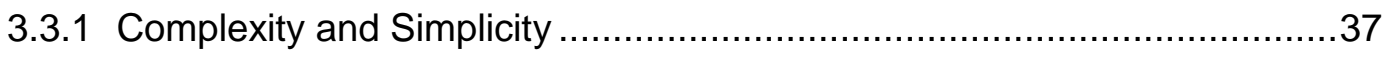

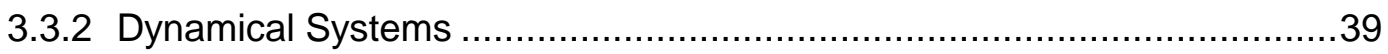

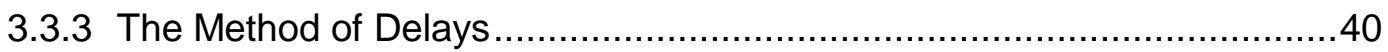

3.3.4 Eigenvalue Decomposition and the Singular Spectrum ..........................43

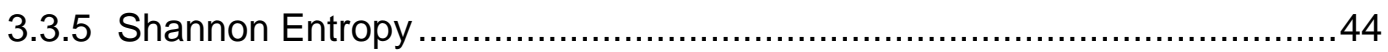

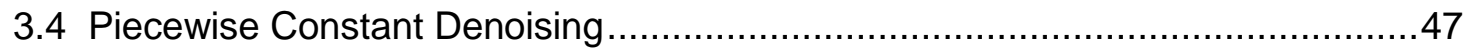

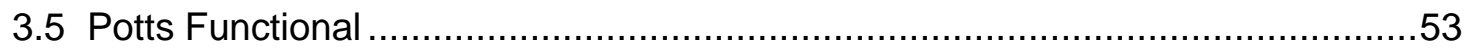

4 SEGMENTATION SYSTEM IMPLEMENTATION …….......................................56

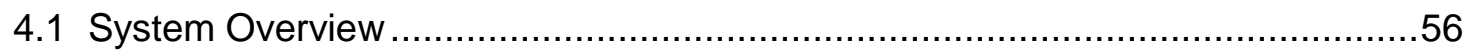

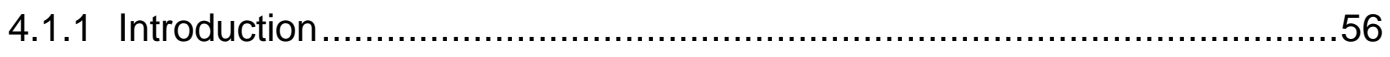

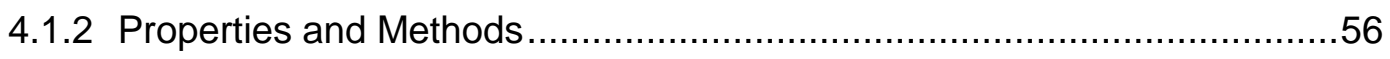

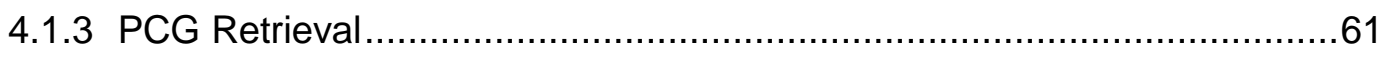

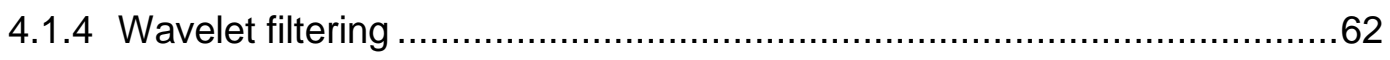

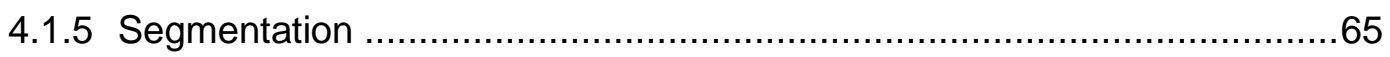

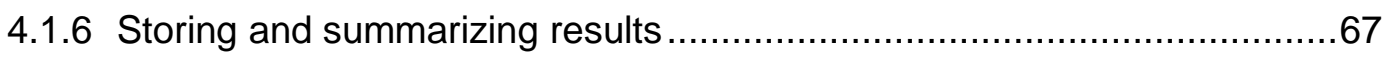

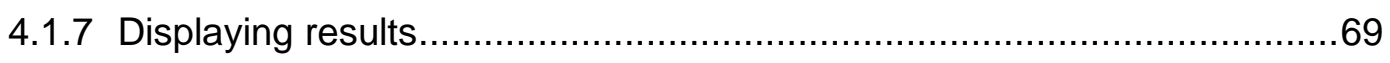

4.2 Wavelet-based Segmentation ................................................................

4.2.1 Heart Sound Segmentation ............................................................

4.2.2 Removing Murmurs from Heart Sound Segments ...................................76

4.2.3 Separating Split Heart Sounds ........................................................

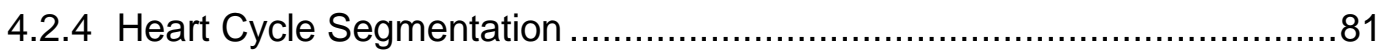

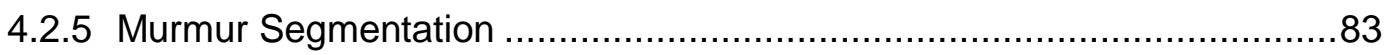


4.2.6 Heart Sound and Murmur Classification

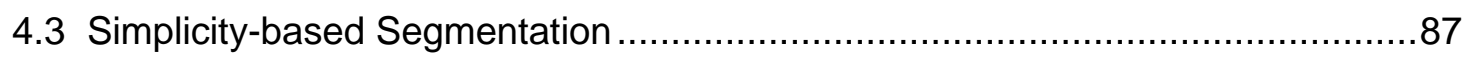

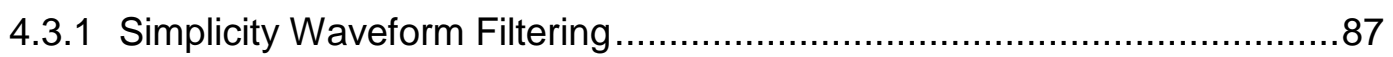

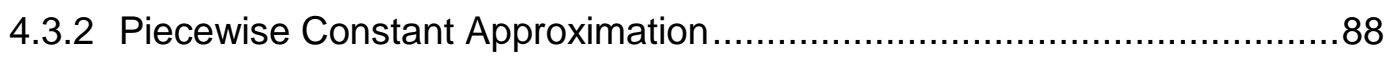

4.3.3 Heart Sound and Murmur Segmentation ..................................... 91

4.3.4 Split Sound Detection, Heart Cycle Segmentation, and Sound

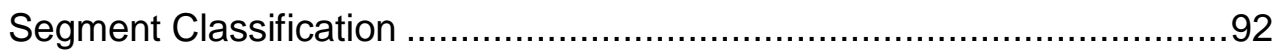

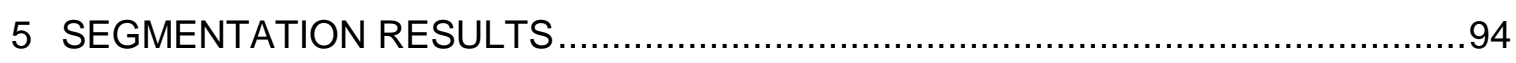

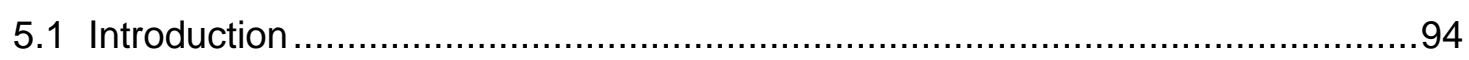

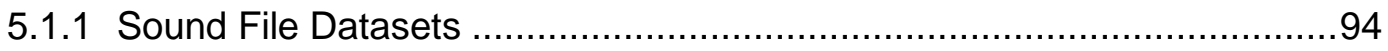

5.1.2 Segmentation Errors and Detection Rates ......................................94

5.1.3 False Negative and False Positive Detection Rates .............................96

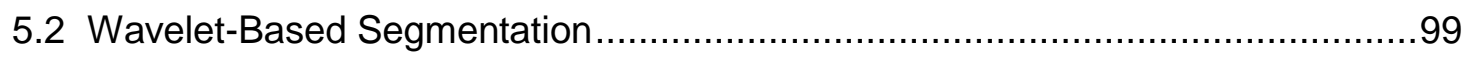

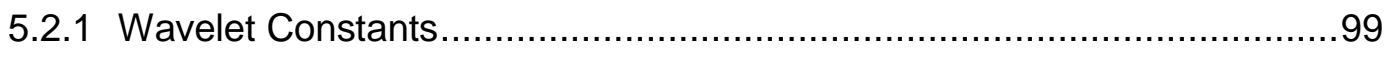

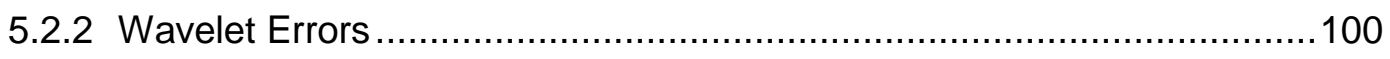

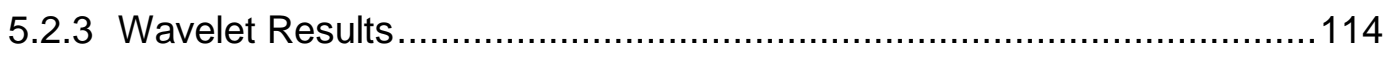

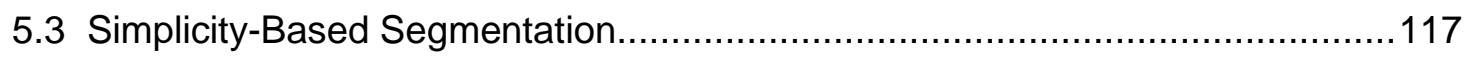

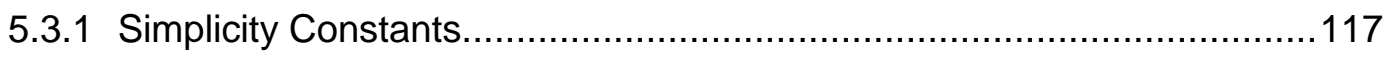

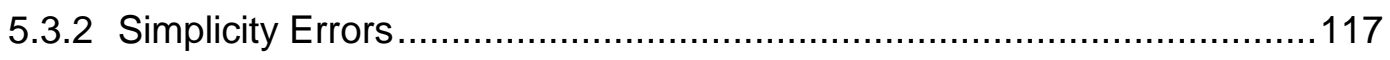

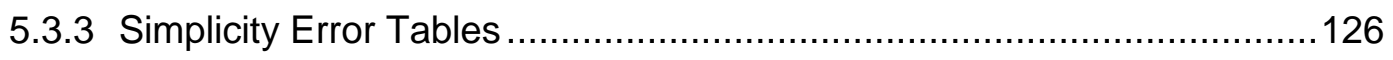

5.4 Comparison of Segmentation Error Performance for the Two Methods............129

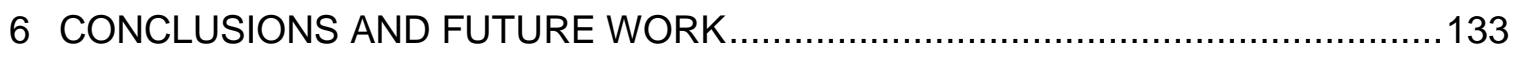

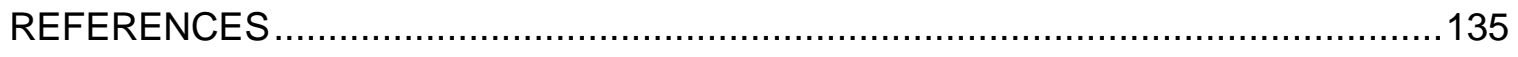

\section{APPENDICES}

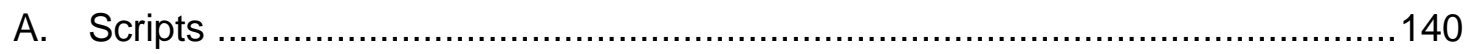

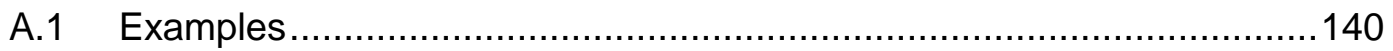




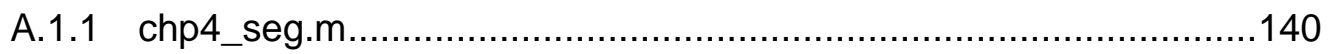

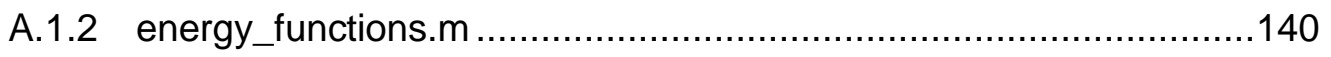

A.1.3 heart_sounds.m................................................................

A.1.4 PCG_FFT.m........................................................................ 142

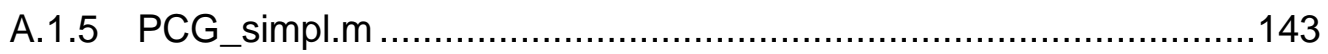

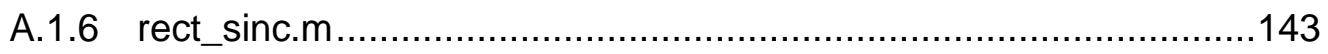

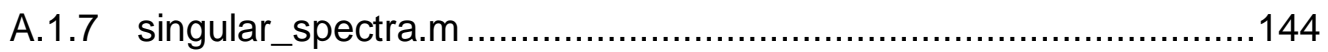

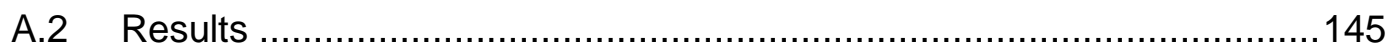

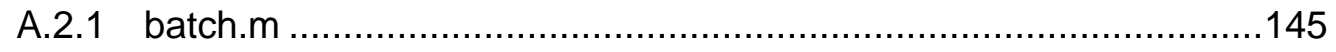

A.2.2 beamforming.m .................................................................

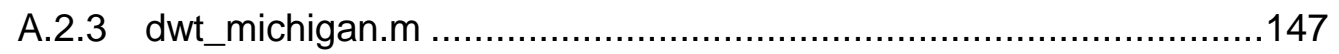

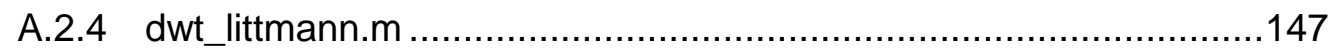

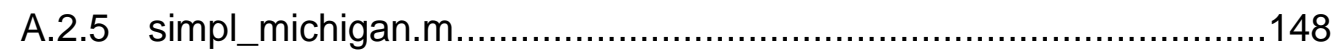

A.2.6 simpl_littmann.m ........................................................ 149

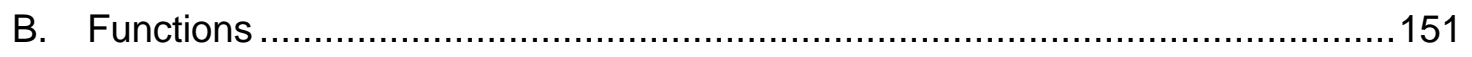

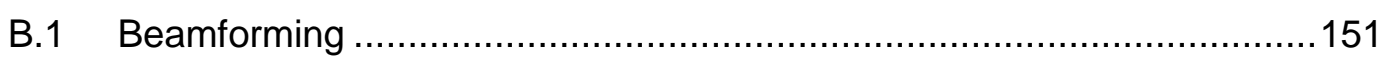

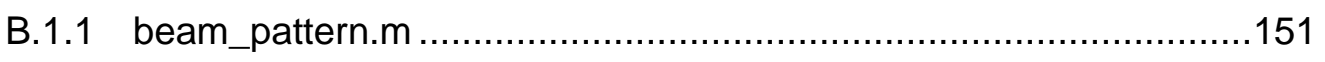

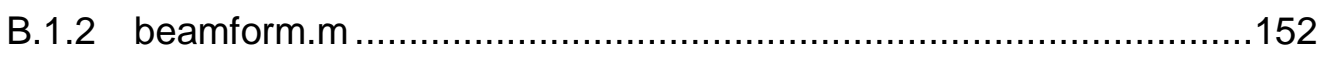

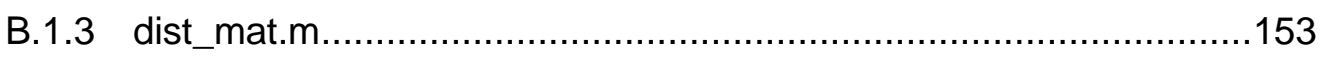

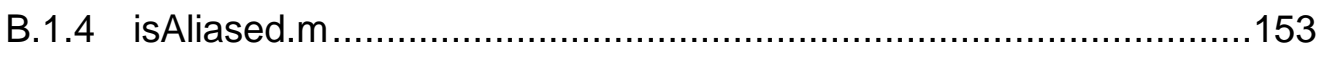

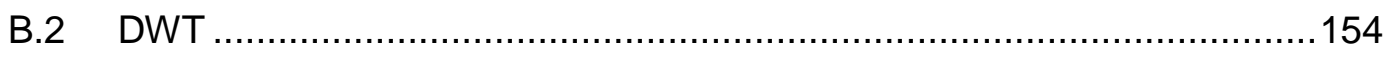

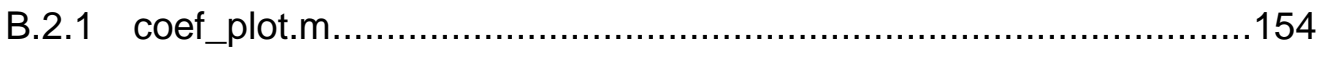

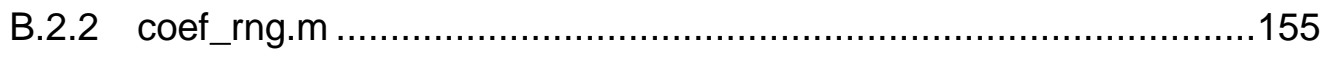

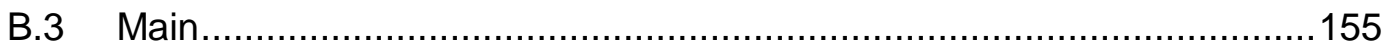

B.3.1 batch_segment.m....................................................... 155

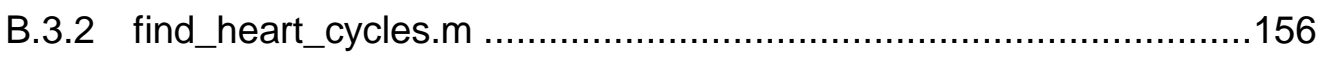




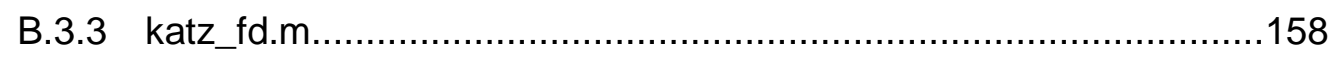

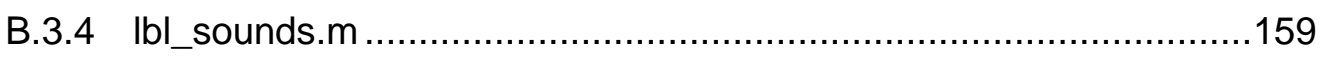

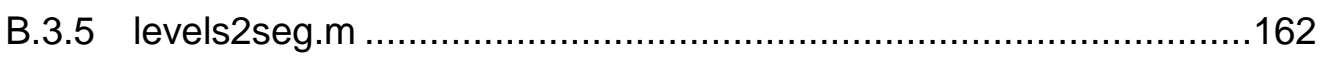

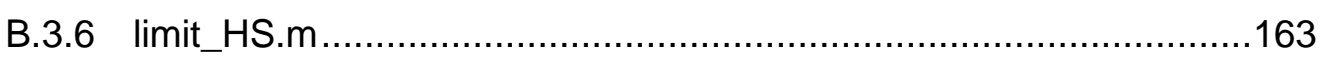

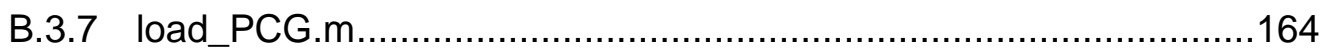

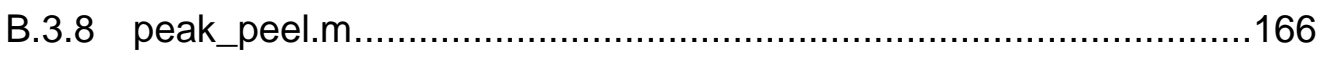

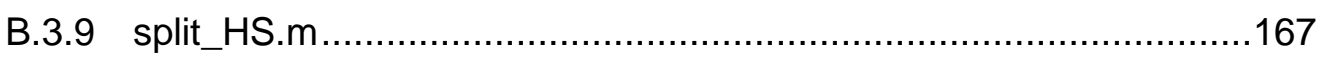

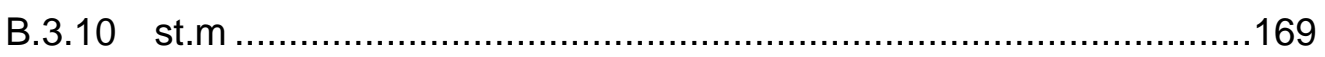

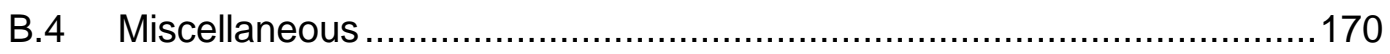

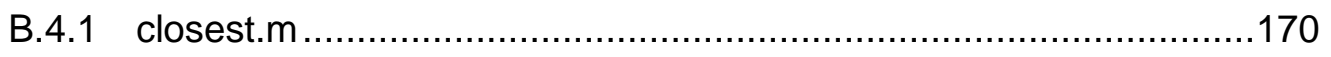

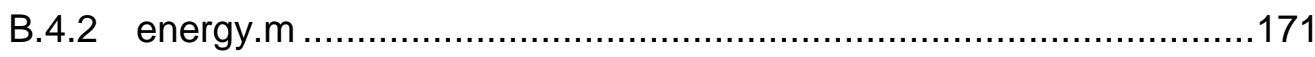

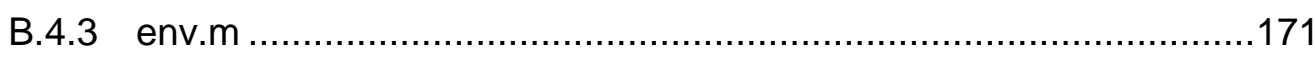

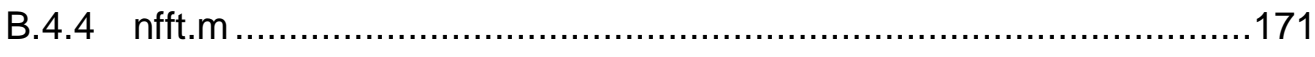

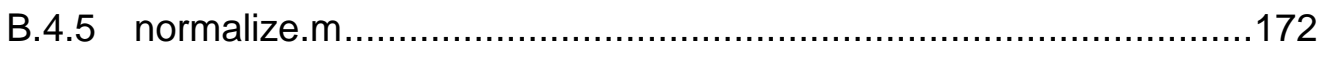

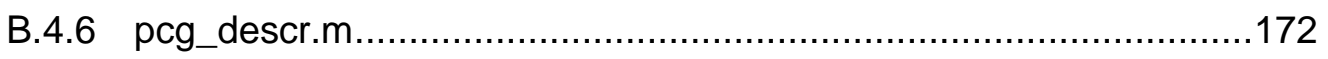

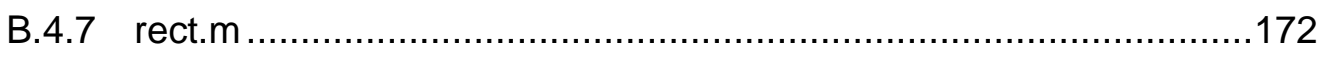

B.4.8 shannon_energy.m................................................................... 172

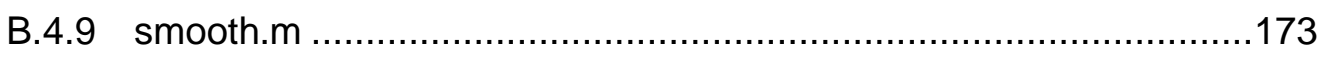

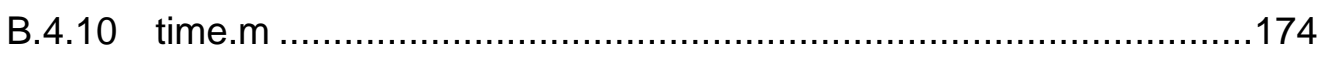

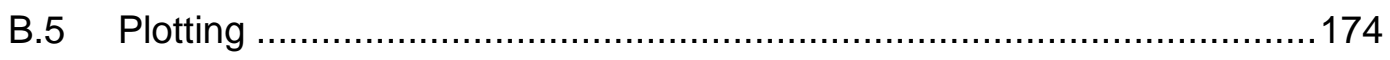

B.5.1 fmt_line_arg.m.......................................................................174

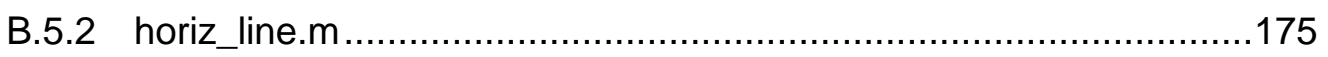

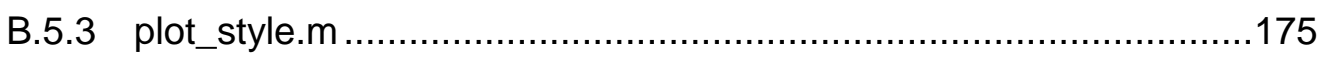

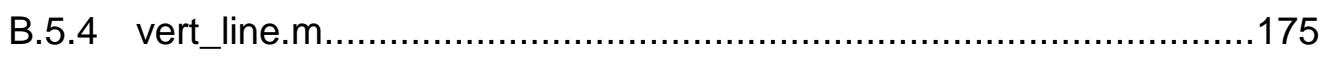

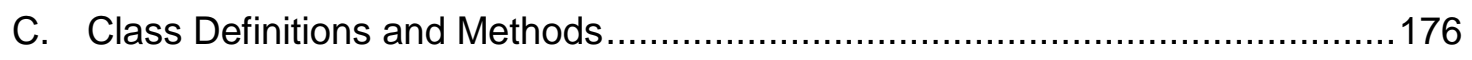

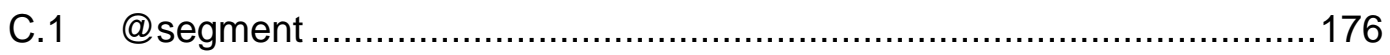




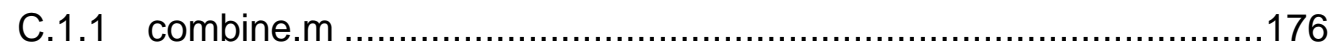

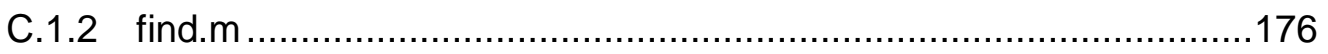

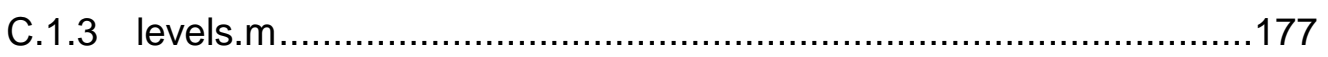

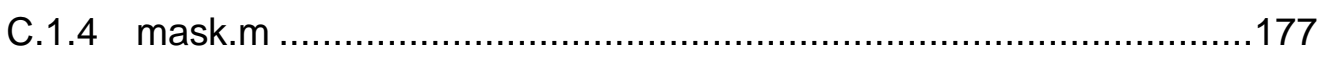

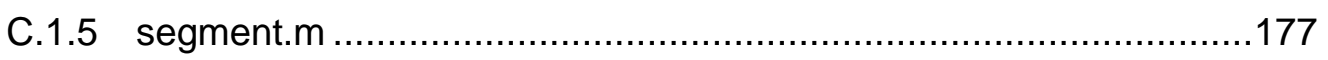

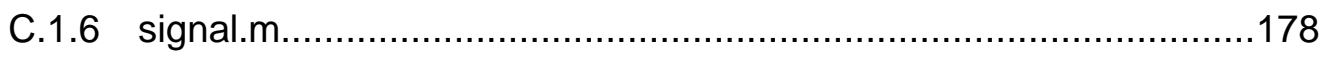

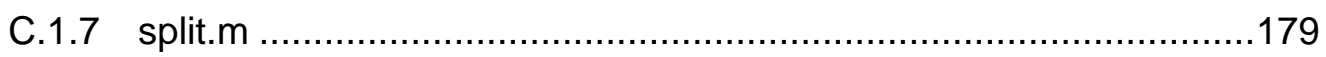

C.2@stethoscope...................................................................... 179

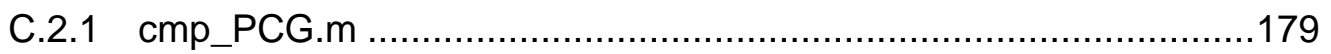

C.2.2 dwt_filt.m................................................................ 180

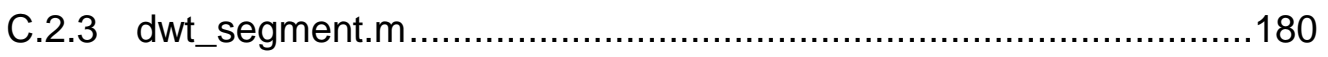

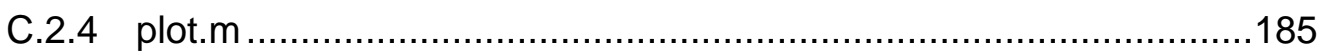

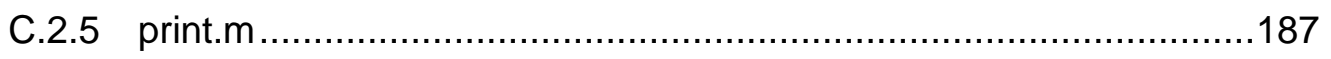

C.2.6 simpl_segment.m ........................................................ 188

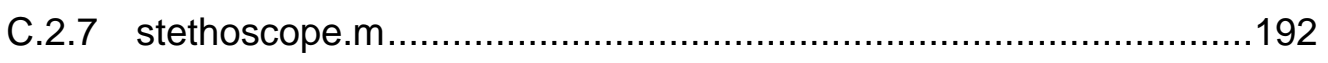

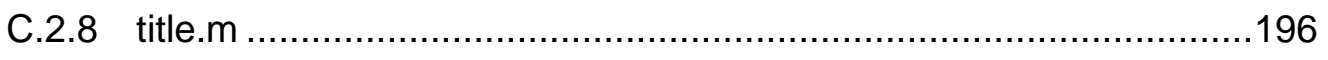




\section{LIST OF TABLES}

Table

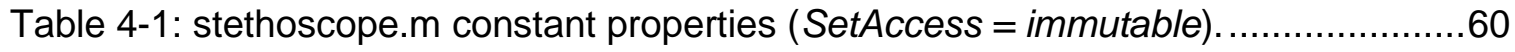

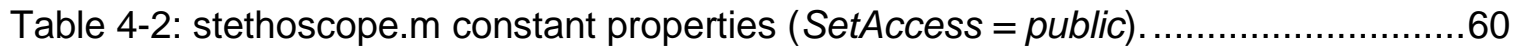

Table 4-3: stethoscope. $\mathrm{m}$ data properties $($ SetAccess $=$ private $) \ldots \ldots \ldots \ldots \ldots \ldots \ldots \ldots \ldots \ldots 1$

Table 4-4: Set of all possible keys for conditions. ............................................68

Table 4-5: Example keys and values for sscope.conditions..................................68

Table 4-6: Heart sound and murmur segment color codes for plot(sscope)..................70

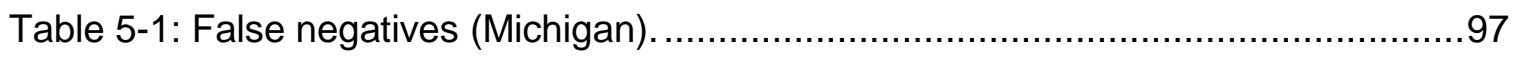

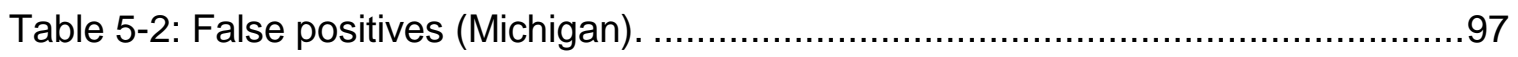

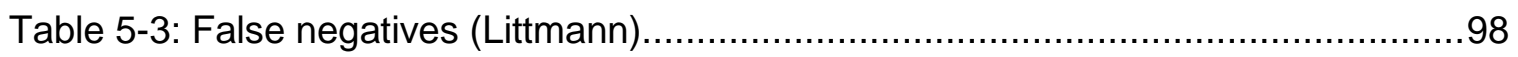

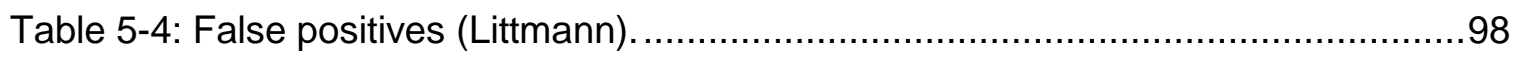

Table 5-5: False negative detection rates (FNDR) .........................................99

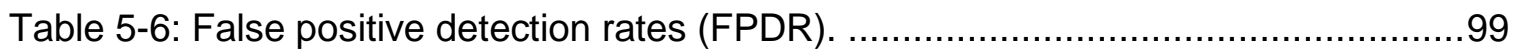

Table 5-7: FNDR and FPDR comparison for wavelet and simplicity-based

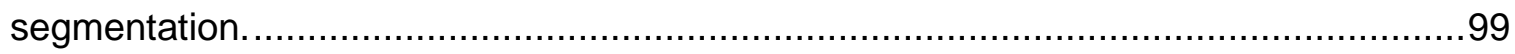

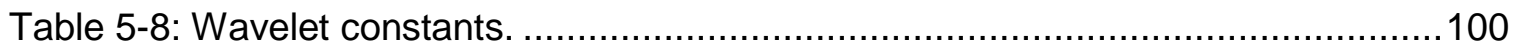

Table 5-9: Wavelet-based segmentation error labels and descriptions. ....................114

Table 5-10: Wavelet-based segmentation results (Michigan) ...............................115

Table 5-11: Wavelet-based segmentation results (Littmann). ..............................116

Table 5-12: Wavelet-based segmentation true murmur detection rate (TMDR). .........116

Table 5-13: Wavelet-based segmentation false murmur detection rate (FMDR).........116

Table 5-14: Simplicity-based segmentation constants. ........................................117

Table 5-15: Simplicity-based segmentation error labels and descriptions. .................126

Table 5-16: Simplicity-based segmentation results (Michigan). ..............................127 
Table 5-17: Simplicity-based segmentation results (Littmann).

Table 5-18: Simplicity true murmur detection rate (TMDR).

Table 5-19: Simplicity false murmur detection rate (FMDR).

Table 5-20: Wavelet and simplicity-based segmentation performance

comparison.

Table 5-21: Wavelet and simplicity-based segmentation error comparisons

(Michigan).

Table 5-22: Wavelet and simplicity-based segmentation error comparisons

(Littmann) 


\section{LIST OF FIGURES}

Figure

Figure 1-1: Blood flow through the heart (oxygen-poor blood is blue, oxygen-rich blood is red). Adapted from [2].... 1

Figure 1-2: The heart's chambers, veins, arteries, and valves [2] ...........................

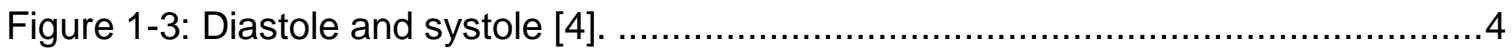

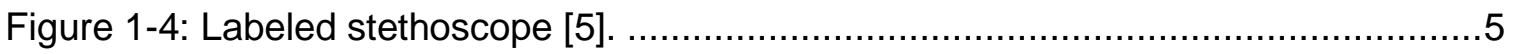

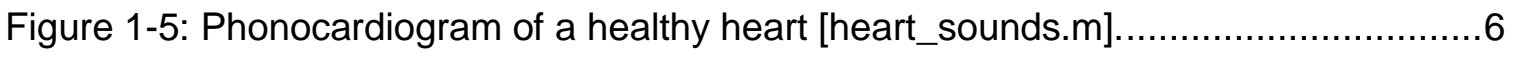

Figure 1-6: PCG with a split S2 [heart_sounds.m] . ..........................................

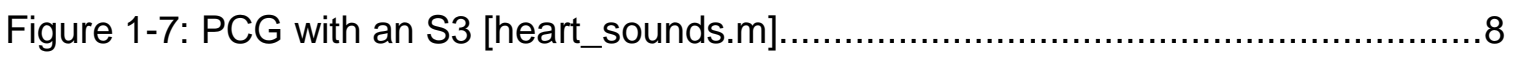

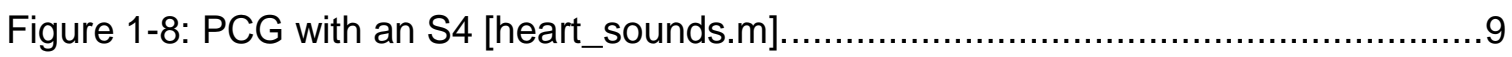

Figure 2-1: Precordial landmarks: Aortic (A), Pulmonic (P), Erb's point $(E)$,

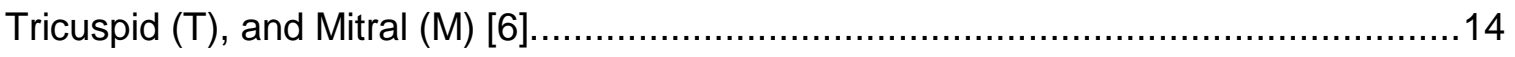

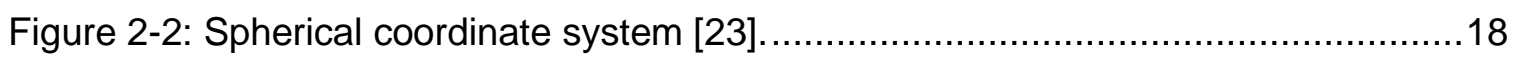

Figure 2-3: Stethoscope positions in the apparatus [beamforming.m] .......................22

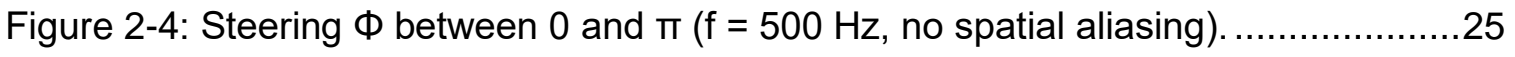

Figure 2-5: Steering $\Phi$ between 0 and $\pi(f=7 \mathrm{kHz}$, no spatial aliasing). ....................27

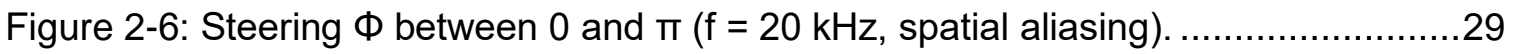

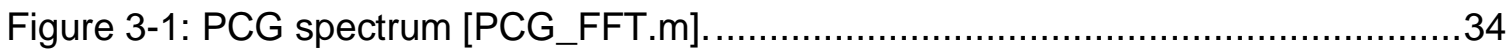

Figure 3-2: Fourier transform of the sinc function [rect_sinc.m] ...............................38

Figure 3-3: Singular spectra comparison [singular_spectra.m]. ..............................44

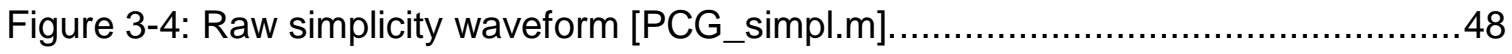

Figure 3-5: Low pass filtering a rectangle causes ripple. The signal at the right was filtered with a higher order LPF than the signal at the left [31]. 
Figure 4-1: A range of approximation coefficients (left subplots) and detail coefficients (right subplots) are used to determine which approximation coefficient is optimal for PCG reconstruction [chp4_seg.m]. 64

Figure 4-2: The original PCG (top subplot) has very little noise, so the filtered PCG (bottom subplot) appears similar to the original PCG [chp4_seg.m].

Figure 4-3: Graphical segmentation results for a PCG with systolic murmurs and split S2 [chp4_seg.m]. 70

Figure 4-4: Shannon energy vs squared energy [energy_functions.m]. 72

Figure 4-5: Two peak peeling iterations. Subplot-1 separates the input signal into the peak signal (blue) and the rejected signal (red). Subplot-2 displays the current output signal, which is a sum of the peaks from the current and previous iterations [chp4_seg.m].

Figure 4-6: PCG (subplot-1), wavelet reconstructed PCG (subplot-2), and peak peeled Shannon energy with overlaid constant threshold (subplot-3) [chp4_seg.m].

Figure 4-7: Final heart cycle and heart sound segment boundaries overlaid on the original PCG (subplot-1), troughs and thresholds for removing murmur samples from heart sound segments (subplot-2), murmur samples removed from heart sound segments (subplot-3), and segmented murmurs (subplot-4) [chp4_seg.m].

Figure 4-8: A heart sound segment containing split heart sounds is separated into its component segments (subplot-3) [chp4_seg.m]. .80

Figure 4-9: The heart cycle boundary locations are approximated from spikes in the autocorrelation of the PCG's envelope (subplot-1). Afterwards, the cycle boundaries are shifted right and aligned with the nearest heart sound segment start indices (subplot-2) [chp4_seg.m]. 
Figure 4-10: The peak peeled fractal dimension extracts the sound peaks from the background noise (subplot-2), which are used to zero the non-sound segments in the raw simplicity waveform (subplot-4) [chp4_seg.m].

Figure 4-11: Threshold the normal heart sounds, extra heart sounds, and murmur segments by their simplicity levels (subplot-2) [chp4_seg.m].

Figure 5-1: The first $\mathrm{S} 1$ is mistaken for a murmur because its maximum energy is less than the energy threshold (subplot-3) [dwt_michigan.m]. 102

Figure 5-2: The first S1 segment is misidentified as a murmur, but the second S1 segment is properly identified (subplot-4). As a result, the first heart cycle's start boundary is moved from S1 to the nearest $\mathrm{S} 2$ (subplot-1) [dwt_michigan.m]. 103

Figure 5-3: The first $S 4$ is misidentified as a murmur because its maximum energy is less than the energy threshold (subplot-3) [dwt_littmann.m]. 105

Figure 5-4: The first S4 is misidentified as a murmur, but the second S4 is acceptably misidentified as a split sound component (subplot-4). The cycle boundary locations are correct because S1 and S2 are properly identified (subplot-1) [dwt_littmann.m]. 106

Figure 5-5: The diastolic murmur is misidentified as a heart sound because its maximum energy is greater than the threshold (subplot-3) [dwt_littmann.m].....

Figure 5-6: The S4 peaks are below the segment thresholds (subplot-2) and are therefore misidentified as murmurs (subplot-4) [dwt_michigan.m].

Figure 5-7: The opening snap murmur is misidentified as a split S2 component because the murmur's peak is above the threshold (subplot-2). Also, the right boundary of $\mathrm{S} 1$ is repositioned despite the lack of a systolic murmur, and the remaining piece is misidentified as a murmur [dwt_michigan.m]. 112

Figure 5-8: The cycle durations are too short because a peak near zero lag is misidentified as a heart cycle boundary [“AP.mp3”, dwt_littmann.m]. 
Figure 5-9: The diastolic murmur is misidentified as a heart sound because its simplicity levels are greater than the HS threshold (subplot-2) [simpl_littmann.m]......119 Figure 5-10: No heart sounds are detected because all segment levels are less than the HS threshold (subplot-5) [simpl_littmann.m] 121

Figure 5-11: The low amplitude diastolic murmurs (not visible) are undetected because they were zeroed while peak peeling the fractal dimension (subplot-2). The corresponding simplicity values are zeroed (subplot-4), so the murmurs are not segmented (subplot-5) [simpl_michigan.m]. .123

Figure 5-12: The summation gallops are misidentified as split sound components because their simplicity levels are less than the extra HS threshold (subplot-2). This causes systole and diastole, and therefore S1 and S2, to be switched (subplot-1) [simpl_littmann.m]. 125 


\section{Introduction}

\subsection{Cardiac Structure and Function}

The purpose of the heart is to circulate blood throughout the body and supply the vital organs with oxygen, route the blood flow through the lungs to enrich the blood with oxygen, and dispose of the $\mathrm{CO}_{2}$ waste collected from the body. The most basic functional breakdown of the heart is to separate it into a right and a left side. Right and left are relative to the observer's own frame of reference, so the orientation is reversed when the heart is presented on a diagram. The right side receives $\mathrm{CO}_{2}$-laden, oxygen-poor blood from the body and sends it to the lungs for $\mathrm{CO}_{2}$ removal and oxygen enrichment. Conversely, the left side receives oxygen-rich blood from the lungs and sends it to the rest of the body for oxygen distribution and $\mathrm{CO}_{2}$ waste collection. This process is synchronous because the left and right sides send and receive blood in unison [1]. The flow of blood through both sides of the heart is illustrated in Figure 1-1.

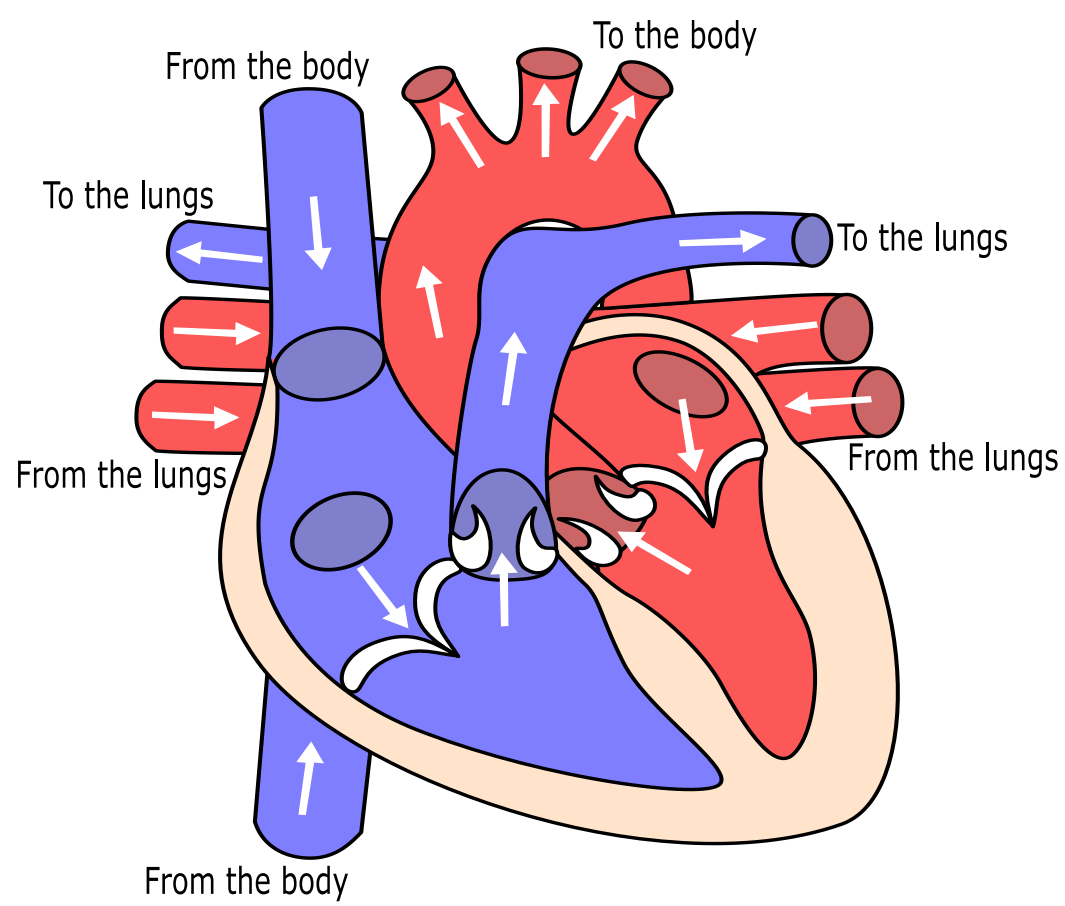

Figure 1-1: Blood flow through the heart (oxygen-poor blood is blue, oxygen-rich blood is red). Adapted from [2]. 
The two sides of the heart are separated by a muscular wall called the septum. Each side of the heart is further subdivided into two chambers, of which there are two types: atria and ventricles. The atria are the upper chambers that collect blood, and the ventricles are the lower chambers that pump blood. The heart has four chambers in total because each side has an atrium and a ventricle [1].

Blood enters the heart through veins and exits through arteries. The right atrium collects $\mathrm{CO}_{2}$-laden waste blood from the body through the venae cavae, where blood from the upper body flows through the superior vena cava, and blood from the lower body flows through the inferior vena cava. The right ventricle then sends the waste blood to the lungs through the pulmonary artery. At the same time, the left atrium collects oxygen-rich blood from the lungs through the pulmonary vein and sends the oxygen-rich blood to the body through the aorta. The heart's chambers, veins, and arteries are labeled in Figure 1-2.

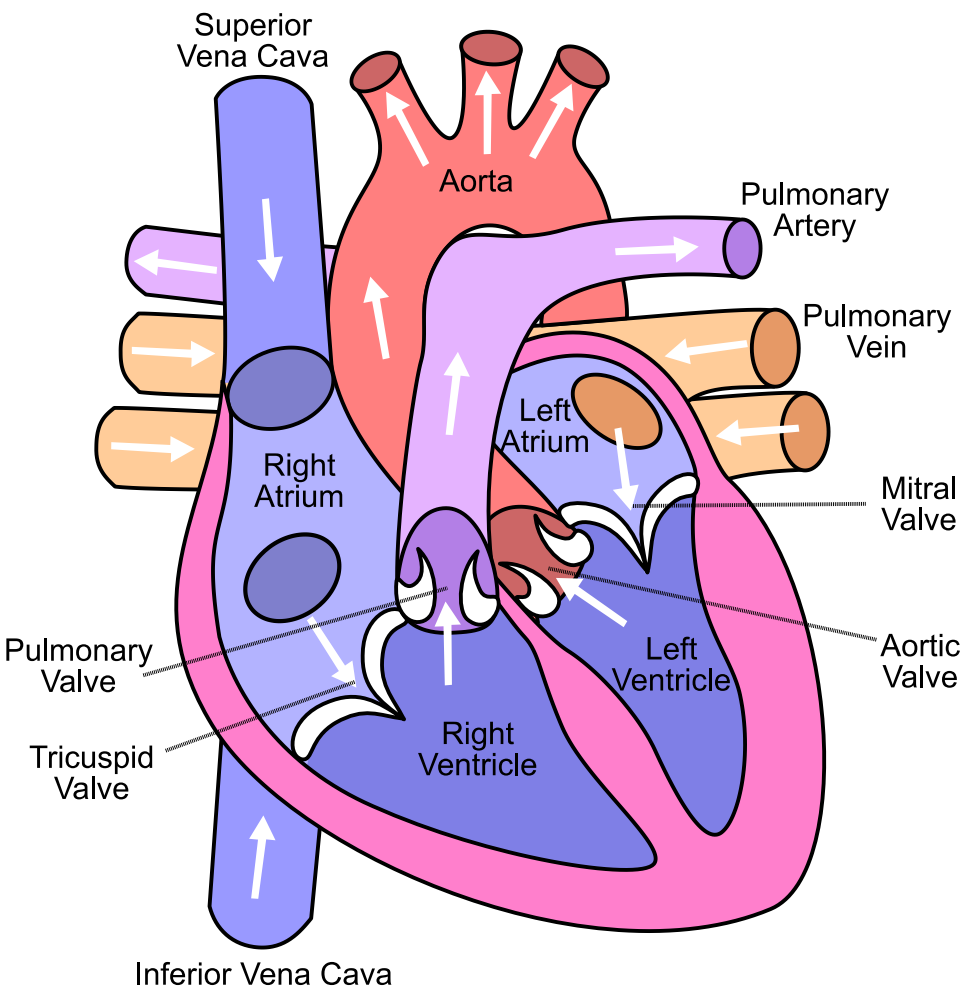

Figure 1-2: The heart's chambers, veins, arteries, and valves [2]. 
The heart is able to pump blood because valves separate the chambers and enable pressure gradients to form. Valves are passive structures made of connective tissue rather than muscle and are shaped like leaflets. The leaflet structure allows pressure differences alone to open or close valves, and it ensures that blood flows in a single direction without backflow into a previous chamber. A valve with leaflets pointing into a chamber will snap shut when the chamber's pressure exceeds the surrounding environment. Likewise, a valve with leaflets protruding from a chamber will open when the chamber's pressure exceeds the surrounding environment.

The atria and ventricles are separated by the atrioventricular valves: the tricuspid valve separates the right atrium and right ventricle, while the mitral valve (bicuspid valve) separates the left atrium and left ventricle. Likewise, the ventricles and arteries are separated by the semilunar valves: the pulmonary valve separates the right ventricle and pulmonary artery, while the aortic valve separates the left ventricle and aorta. The four valves are labeled in Figure 1-2.

\subsection{Cardiac Cycle}

The cardiac cycle is divided into two distinct phases: diastole and systole. Diastole occurs when the ventricles relax and blood fills the atria (filling phase), while systole occurs when the ventricles contract and pump blood into the arteries (ejection phase) [3].

Diastole begins after the ventricles expel blood into the arteries, and the semilunar valves snap shut due to the arterial pressure exceeding ventricular pressure. At the same time, atrial pressure exceeds ventricular pressure, so blood returning from the body flows through the atrioventricular valves and fills the ventricles. The majority of the blood reaches the ventricles passively, but the atria eventually contract and force any remaining blood into the ventricles. 
Systole begins after the ventricles completely fill with blood, and the atrioventricular valves snap shut due to the ventricular pressure exceeding the atrial pressure. The semilunar valves are already shut from diastole, so the ventricles begin contracting to rapidly increase their pressure. When ventricular pressure exceeds arterial pressure, the semilunar valves snap open, and blood is ejected from the ventricles into the arteries. In a healthy heart, systole is shorter than diastole because the ejection phase is much quicker than the filling phase. A blood flow for diastole and systole is illustrated in Figure $1-3$.

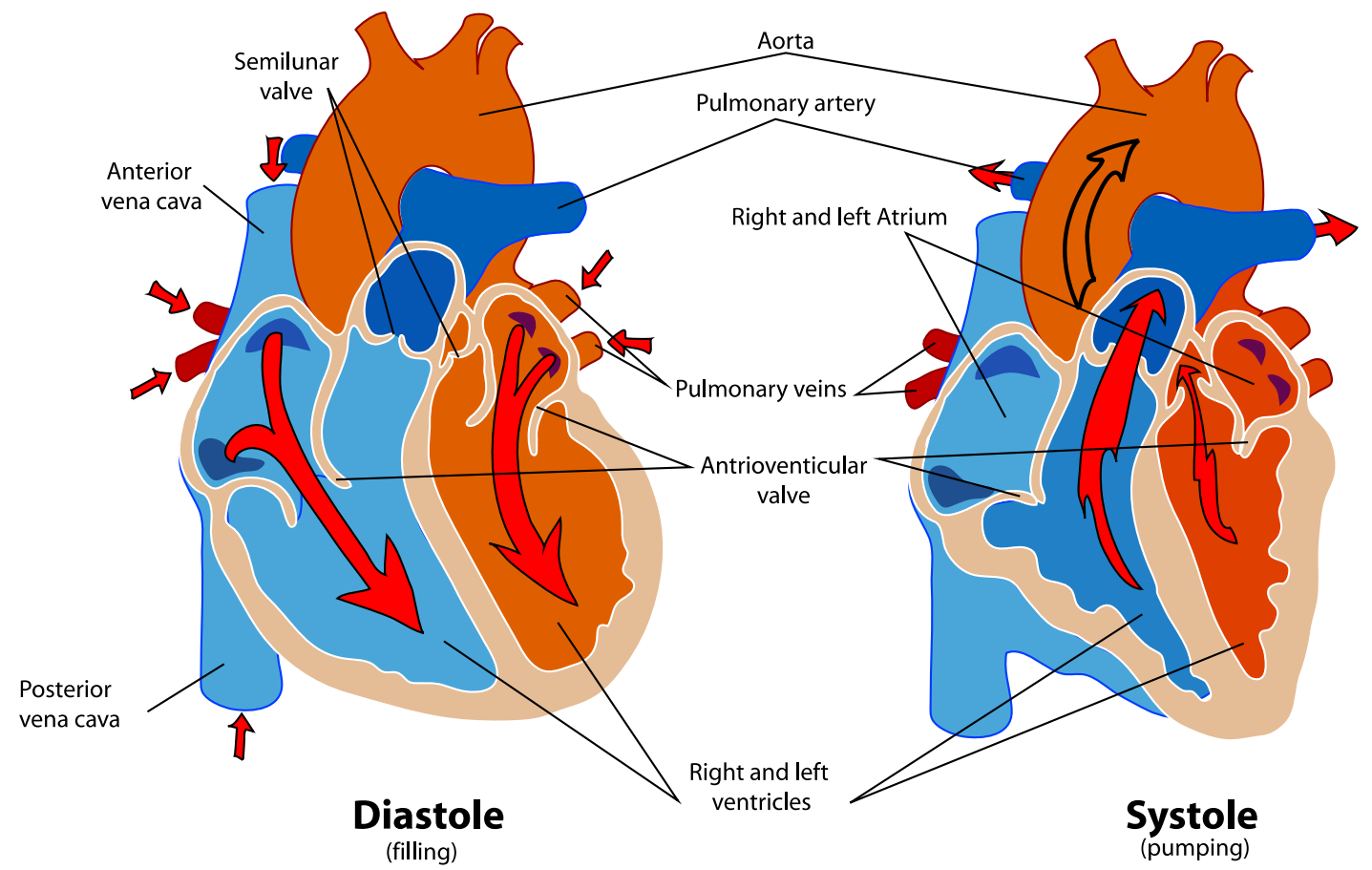

Figure 1-3: Diastole and systole [4].

\subsection{Auscultation, Heart Sounds, \& Murmurs}

Auscultation is the act of listening to internal body sounds [5] and is performed with a stethoscope when listening for heart sounds. The stethoscope's two-sided chestpiece has the bell and diaphragm acoustic pickups (Figure 1-4). The diaphragm has the larger 
circumference and is used for listening to higher pitched sounds, while the bell has the smaller circumference and is used for listening to lower pitched sounds [5]. However, modern stethoscopes often have a tunable diaphragm, instead of a bell, which can be adjusted for both low and high pitched sounds.

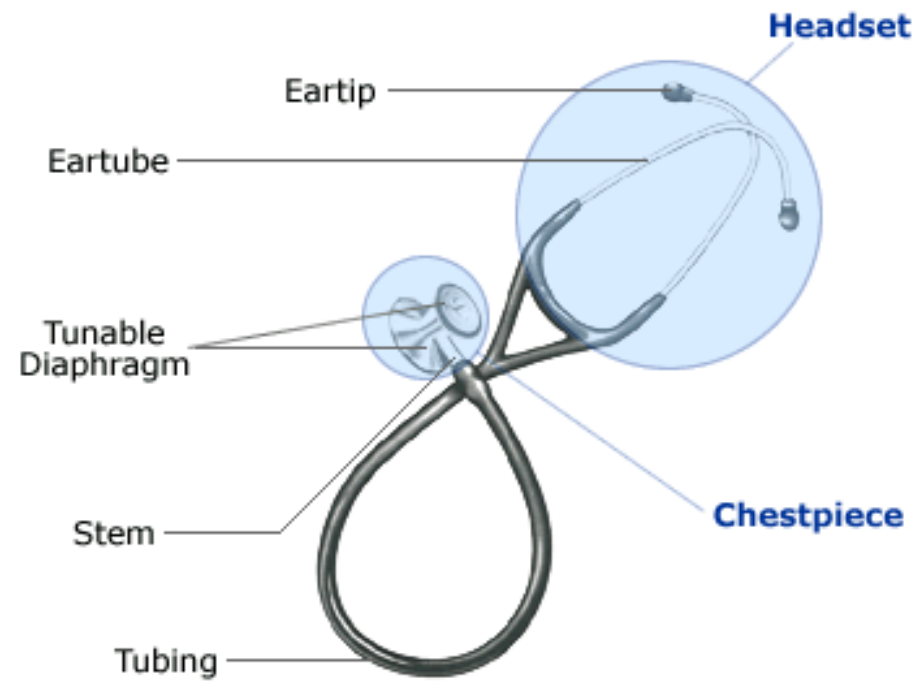

Figure 1-4: Labeled stethoscope [5].

Normal heart sounds associated with systole and diastole are audible during auscultation when closing heart valves vibrate against the chambers of the heart and radiate sound throughout the chest (opening valves are inaudible) [1]. The first normal heart sound, $S 1$, occurs at the beginning of systole when the atrioventricular valves snap shut; and the second normal heart sound, S2, occurs at the beginning of diastole when the semilunar valves snap shut. In addition to the normal heart sounds, extra heart sounds may occur during diastole. If blood strikes a non-compliant left ventricle during passive filling, then an extra S3 sound occurs shortly after the normal S2; and if the blood ejected by the left atrium at the end of diastole also strikes a non-compliant left ventricle, then an extra S4 sound occurs shortly before the S1 that starts the next systole phase.

Unlike heart sounds, murmurs are sounds that are caused by the disruption of laminar blood flow rather than the closing of heart valves, and are induced through four primary 
means: narrowing of valves (stenosis), backflow through bad valves (valve insufficiency or "regurgitation"), irregular flow between chambers (septal defect), and high volume flow [6]. Murmurs are typically named after the heart valve or chamber where the defect occurs, for example: aortic stenosis (AS), mitral regurgitation (MR), atrial septal defect (ASD), etc. For this thesis, describing the murmurs as either systolic or diastolic is sufficient.

A phonocardiogram (PCG) is a recording of a heart sound's intensity over time [7], which is illustrated in Figure 1-5 for a healthy heart. A PCG makes it possible to algorithmically detect and classify the heart sounds and murmurs.

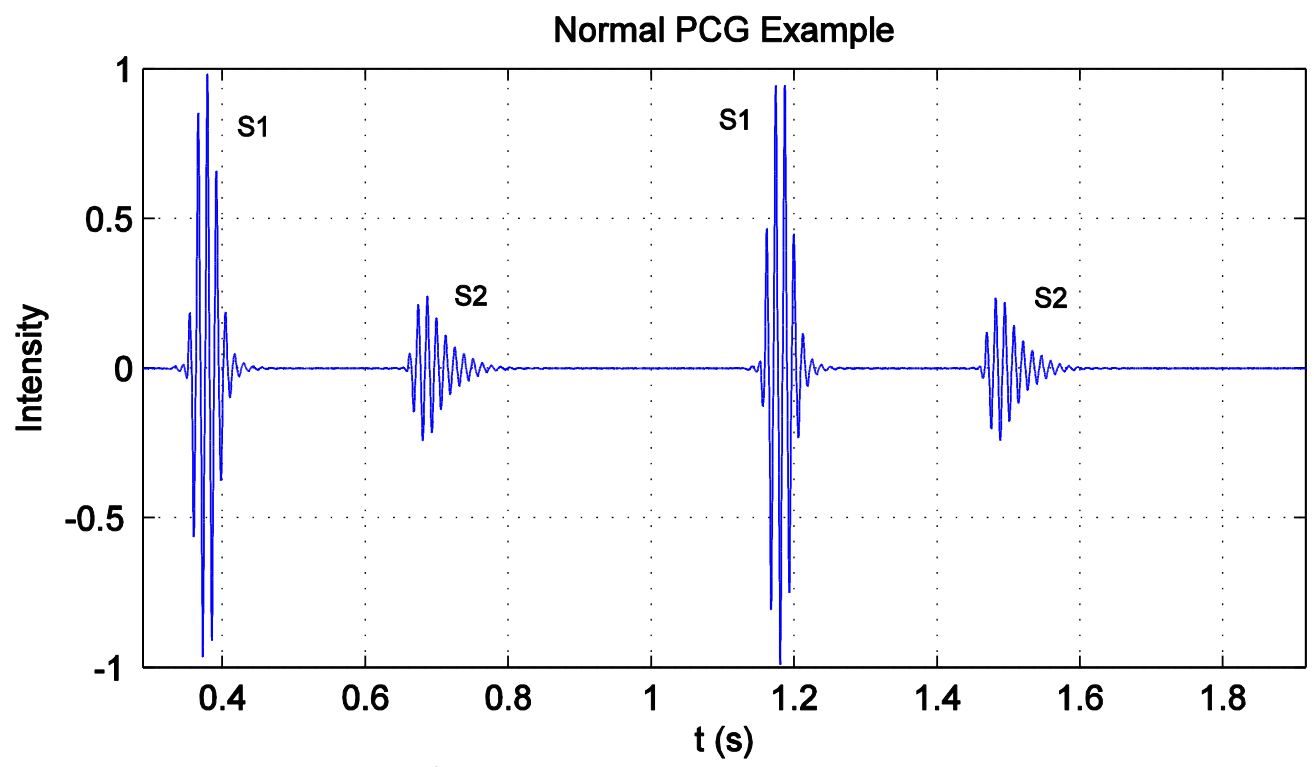

Figure 1-5: Phonocardiogram of a healthy heart [heart_sounds.m].

In a phonocardiogram, S1 is typically louder (higher amplitude) than S2 due to the higher pressure on the left side of the heart. However, the relative intensities are sometimes switched, particularly in the elderly, so this feature cannot be used to reliably distinguish S1 from S2. Instead, systole and diastole are determined by comparing the distances between unidentified heart sounds, where systole is shorter than diastole because blood ejects from the ventricles more rapidly than it fills the ventricles. Since S1 
is the beginning of systole, and S2 is the beginning of diastole, S1 and S2 can therefore be identified by their temporal locations and spacing rather than by their intensities.

Normal heart sounds are in fact the superposition of two sound components generated by a valve closing on each side of the heart. Therefore, a split heart sound occurs when it is possible to audibly or visually distinguish the two independent sound components resulting from each individual heart valve closure, which can be seen in Figure 1-6 [8]. For a split $\mathrm{S1}$, the mitral valve (M1) closes before, and is louder than, the tricuspid valve (T1); and for a split S2, the aortic valve (A2) closes before, and is louder than, the pulmonic valve (P2). A physiological split occurs when the two sound components constituting S1 or S2 are audible during inspiration, but are inaudible otherwise, which is a common occurrence in healthy individuals and does not necessarily indicate heart dysfunction on its own. However, split sounds might indicate dysfunction when the split is persistent, regardless of inspiration, or when the first split component has a lower intensity than the second split component.

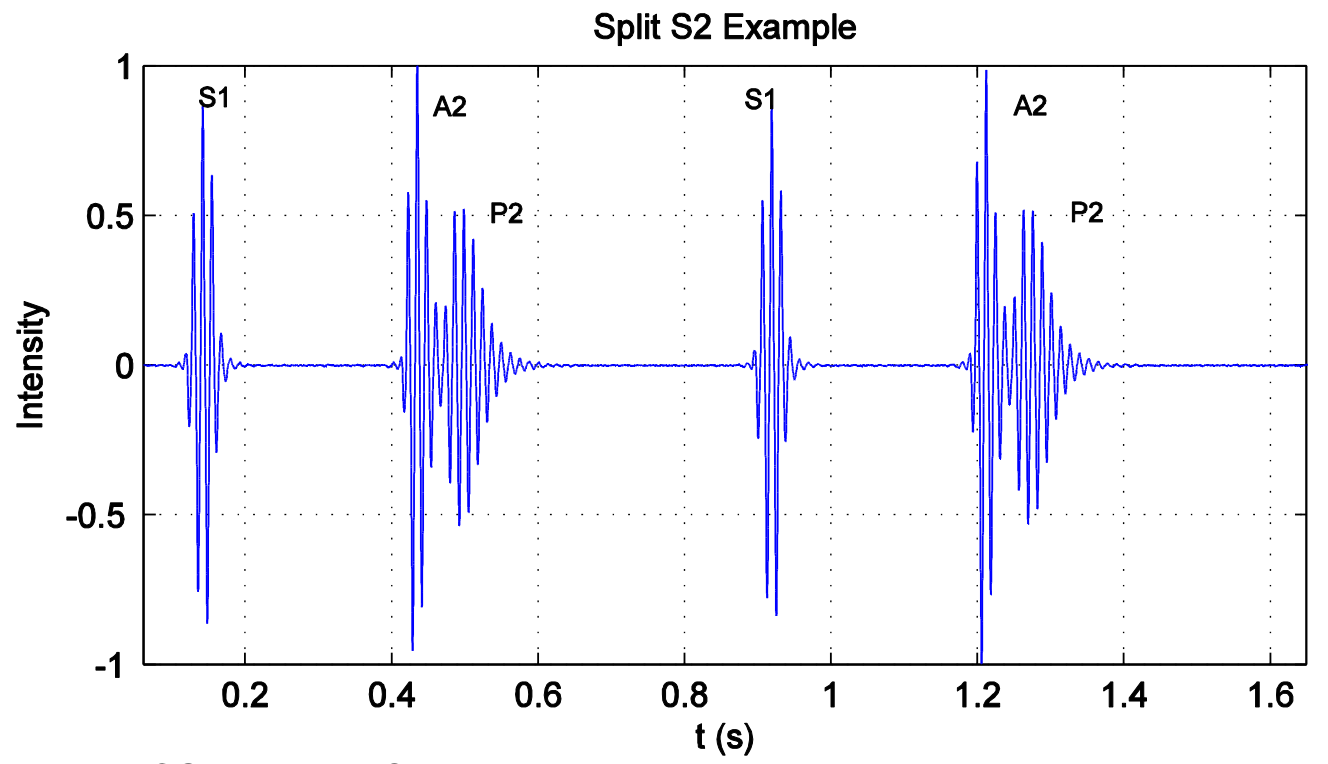

Figure 1-6: PCG with a split S2 [heart_sounds.m]. 
Since S3 occurs shortly after S2, and S4 occurs shortly before S1, it is often difficult to distinguish S3 and S4 from split sound components. However, both of these sounds typically have lower frequencies and lower intensities than S1 and S2, so it is possible to identify them through careful auscultation or visual analysis of the PCG. S3 can be seen in Figure 1-7, and S4 can be seen in Figure 1-8. It is also important to note that $\mathrm{S} 3$ and $\mathrm{S} 4$ are ignored when determining $\mathrm{S} 1$ and $\mathrm{S} 2$ by comparing the distances between unidentified normal heart sounds.

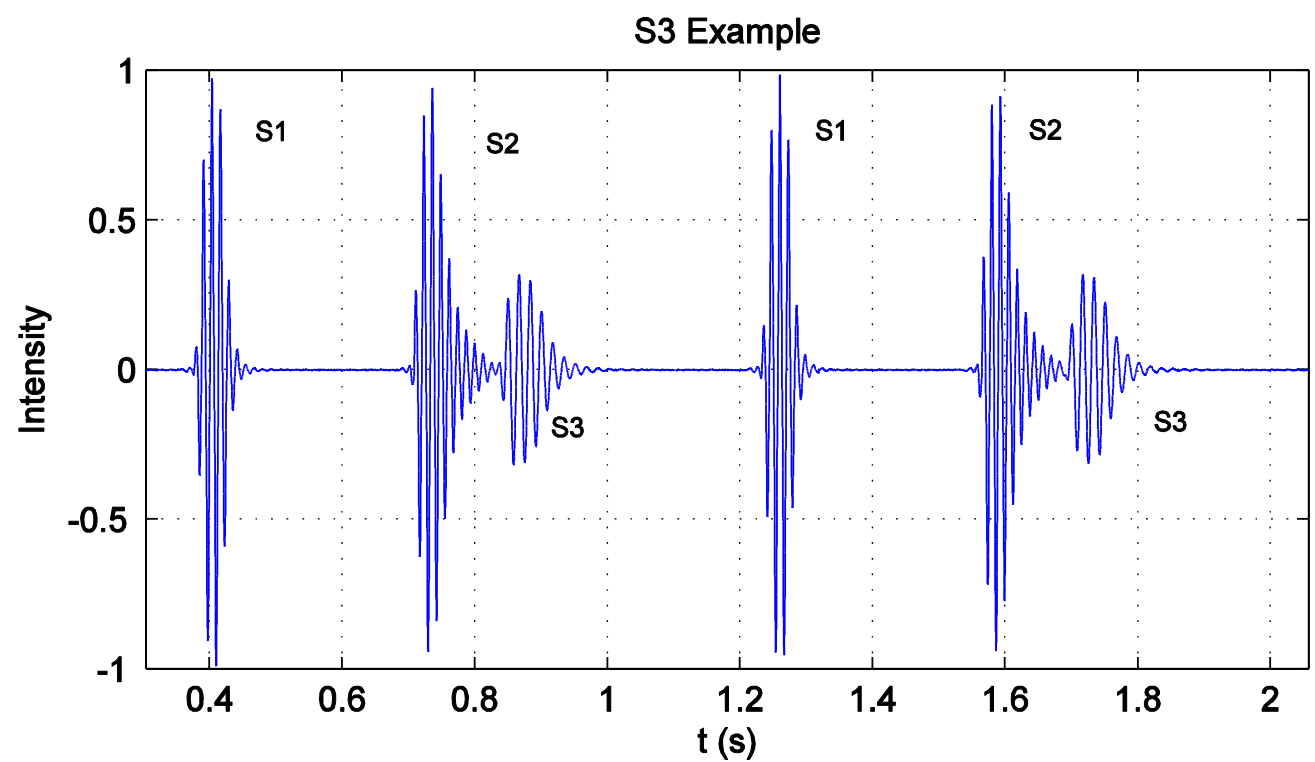

Figure 1-7: PCG with an S3 [heart_sounds.m]. 


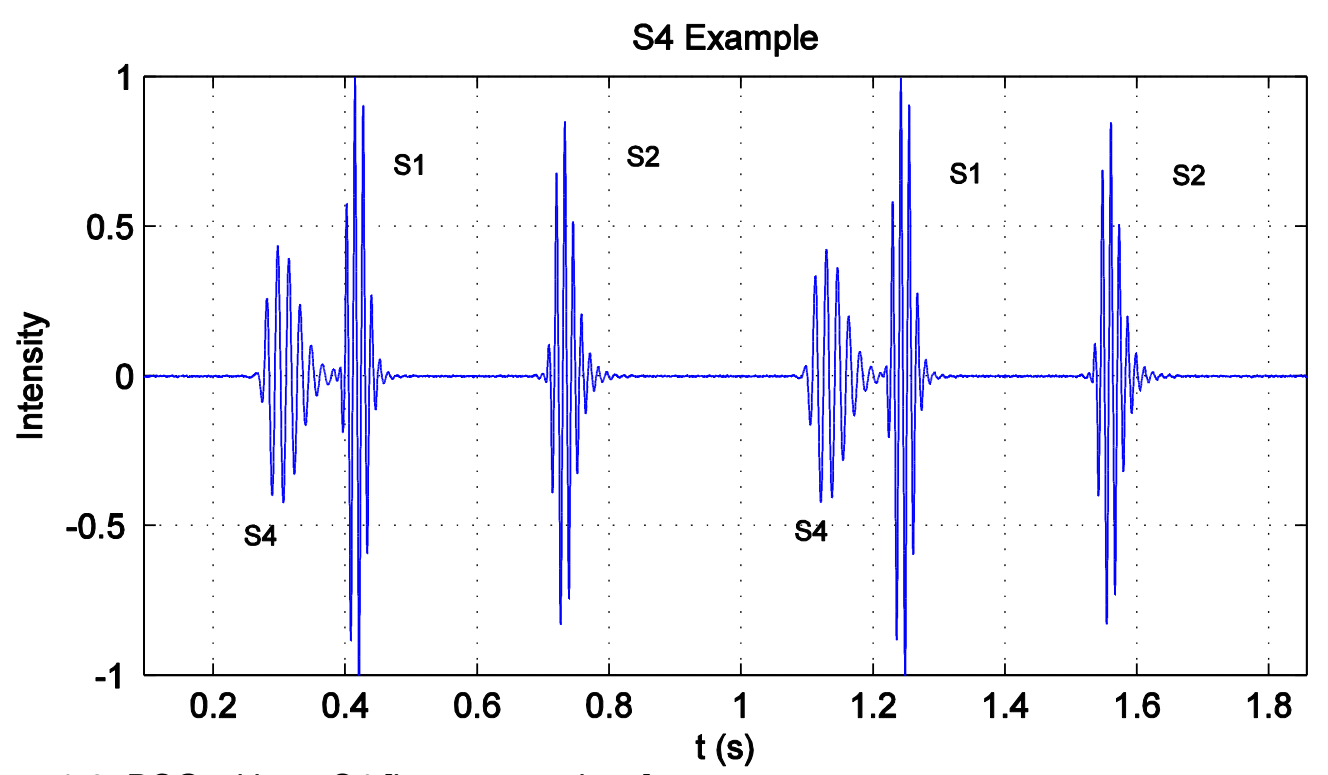

Figure 1-8: PCG with an S4 [heart_sounds.m].

Murmurs are distinguishable from heart sounds because they have higher pitches and are irregularly shaped compared to heart sounds. If murmurs are present, they exist in the time periods between S1 and S2, and are therefore classified as either systolic or diastolic. They are further categorized by their relative durations and locations within systole or diastole as either early, mid, late, or holo-systolic/diastolic murmurs ("holo" murmurs occupy the entire systole or diastole).

\subsection{Heart Sound and Murmur Segmentation Goals}

The primary purpose of this thesis is to improve the accuracy and efficiency of established techniques for detecting and segmenting heart sounds and murmurs. Since the sounds are nonstationary events, the first challenge is distinguishing sound segments from background noise, which is accomplished through a process known as peak peeling. In general, the peaks extracted through peak peeling do not necessarily represent a single sound segment since heart sounds and murmurs are often merged into a single peak. For example, ejection murmurs are initiated shortly after $\mathbf{S 1}$, and holosystolic murmurs span the entirety of systole, so both of these murmurs blur the boundaries between heart 
sounds and murmurs. Also, split heart sounds with multiple peaks are sometimes difficult to detect through peak peeling alone. As a result, additional techniques must be developed for accurately segmenting heart sounds and murmurs, irrespective of their proximities to other sounds.

In addition to locating the segment boundaries, this thesis attempts to classify the specific type of each sound segment. Central to this objective is using the normal heart sounds S1 and S2 as markers for locating the heart cycle boundaries and distinguishing systole from diastole. In particular, the heart cycle boundaries are located by cross correlating the signal with itself (autocorrelation) [9] and then aligning the boundaries with the nearest S1 or S2 segment for greater accuracy. Isolating the heart cycles allows for systole and diastole, and hence $\mathrm{S} 1$ and $\mathrm{S} 2$, to be identified on a cycle-by-cycle basis. Finally, the murmurs can be located within systole or diastole and classified accordingly.

\subsection{Literature Review}

Various methods have been established for detecting heart sounds and murmurs, but this thesis in particular extends established wavelet and simplicity-based segmentation techniques.

The peak peeling algorithm introduced by Hadjileontiadis and Rekanos [10] [11] was developed for the purpose of extracting explosive lung and bowel sound segments from the background noise. It is most effective at detecting these transient sound peaks when applied to the fractal dimension of the PCG, which is a positive-valued signal that is a measure of time domain complexity. In particular, the fractal dimension attenuates noise significantly (including noise that is typically unfilterable through standard linear

processing techniques) but transforms the actual sounds into prominent peaks. Peak peeling is sufficient for accurately segmenting explosive lung and bowel sound peaks due to their characteristic crescendo-decrescendo shape, which tends to produce distinct start 
and stop boundaries. When multiple sounds are merged into a single peak, a second peak peeling iteration is typically sufficient for separating the sounds, as the crescendodecrescendo shape tends to produce a deep, distinct trough, even between merged peaks. Peak peeling is likewise effective at segmenting normal heart sounds given their similar morphology to explosive lung and bowel sounds. However, it is ineffective at detecting merged heart sounds and murmurs because murmurs that begin immediately after S1 or S2 do not produce a deep enough trough for a second iteration to reliably separate the peaks.

A rudimentary wavelet-based segmentation technique is proposed by Atanasov and Ning [12]. The purpose of the discrete wavelet transform (DWT) here is to attenuate the higher frequency murmurs but not the lower frequency heart sounds. The peaks are then analyzed in the filtered PCG's energy waveform, where the non-attenuated peaks are identified as heart sound segments. In practice, the attenuated murmurs will have a small, but non-zero, energy value, so a threshold is used to distinguish peaks from non-peaks. After segmenting the heart sounds, the unfiltered PCG's energy waveform is used to segment the murmurs. This straightforward approach is acceptable as long as the murmurs are attenuated sufficiently, which is the case here because the demonstrated murmurs exhibit ideal attenuation.

The simplicity transform detailed by Nigam and Priemer [13] forms the basis of an amplitude and energy-invariant segmentation technique that is implemented by Kumar et al [14]. The simplicity is an inverse measure of signal complexity that is obtained by embedding the time domain signal into a higher dimensional state space representation, so that the state space dimension can be used to estimate signal complexity. Unlike the fractal dimension, each sound segment has an approximately constant simplicity value, or level, which allows for accurate identification of segment boundaries and sound type. 
In particular, simplicity-based segmentation can distinguish between heart sounds, murmurs, and noise because heart sounds have higher simplicity levels than murmurs, while noise has the lowest possible simplicity level.

\subsection{Proposed Modifications to the Established Methods}

The wavelet-based segmentation method proposed by Atanasov and Ning is problematic because it assumes that all murmurs are sufficiently attenuated after filtering, so that each peak encompasses a single heart sound segment. However, some of the murmurs examined in this thesis are only partially attenuated after filtering, so certain peaks may only contain murmurs, while others may contain both heart sounds and murmurs. Therefore, three improvements are proposed for wavelet-based segmentation. The first applies a threshold to the filtered energy waveform to remove any partially attenuated, low energy murmur peaks. The second searches the remaining peaks for troughs that might indicate merged heart sounds and murmurs, and if found, removes the murmurs. The third uses peak peeling to detect the heart sound and murmur segment boundaries with greater accuracy. In particular, the heart sounds are segmented by peeling the filtered energy waveform, while the murmurs are segmented by peeling the fractal dimension of the original PCG.

The simplicity-based segmentation method proposed by Kumar et al, despite offering a marked performance improvement over wavelet-based segmentation, is also problematic in certain regards. This is primarily a result of the simplicity waveform's imperfect resemblance to a piecewise constant function, where the simplicity values in each segment do not form a constant level, and the transitions between levels are not instantaneous. This is acceptable when the sound segments are disconnected, as each segment's level can be approximated by its average simplicity value, but is ineffective when heart sounds and murmurs are merged into a single peak. Instead, the simplicity 
waveform's piecewise constant approximation can be determined through a process known as piecewise constant denoising, the theory of which is detailed by Little and Jones [15]. The particular denoising algorithm used in this thesis is implemented in the MATLAB toolbox Pottslab by Storath et al [16] [17] [18]. Another drawback of simplicity-based segmentation is the computational cost incurred by embedding the time domain signal into state space, which involves multiplying matrices that are proportional in size to number of samples in the analyzing window. Since the sound segments are only intermittent events, calculating the simplicity for all samples in the PCG is inefficient. In this thesis, the sound peaks are first extracted from the background noise by peak peeling, so that the simplicity only has to be calculated within sound segment boundaries. 


\section{Viability of Using a Stethoscope Array for Improved Heart Sound Detection}

\subsection{Introduction}

A stethoscope is a single element passive acoustic sensor that receives sound waves from the body. Heart sounds and murmurs are best heard when the chestpiece of the stethoscope is placed on one of five precordial landmarks on the chest (Figure 2-1). The aortic $(A)$, pulmonic $(P)$, tricuspid $(T)$, and mitral $(M)$ landmarks are the optimal listening locations for their associated valves. Thus, S1 is best heard at the tricuspid and mitral areas, while S2 is best heard at the aortic and pulmonic areas. The fifth landmark, Erb's point (E), evenly splits the sound between S1 and S2 [19].

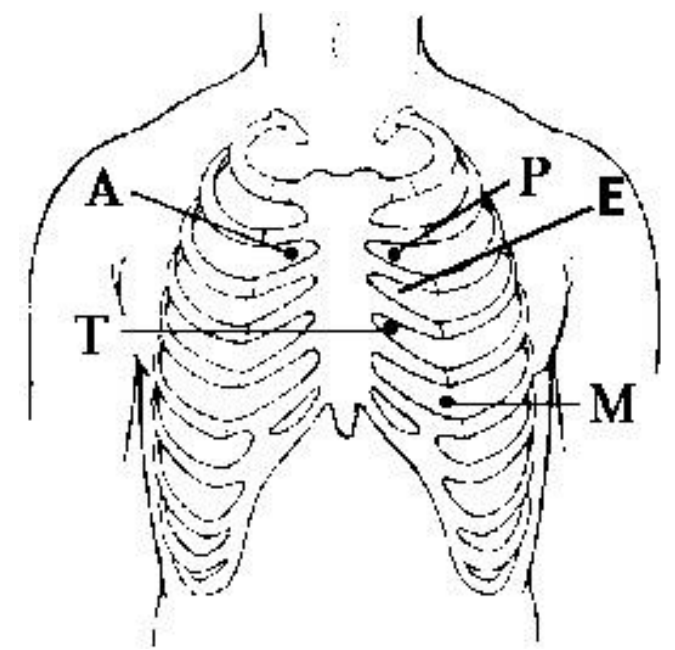

Figure 2-1: Precordial landmarks: Aortic (A), Pulmonic (P), Erb's point $(E)$, Tricuspid $(T)$, and Mitral (M) [6].

The five precordial landmarks are optimized for auscultating certain murmurs but are nonetheless corrupted by sounds from other locations in the heart and the rest of the body.

\subsection{Multiple Input Stethoscope}

A multiple input stethoscope is proposed by Wong in [20] to improve the signal to noise ratio (SNR) of a PCG. Of interest here is whether or not such a multi-sensor configuration could provide an improved PCG waveform for segmentation and heart sound 
detection by applying beamforming techniques to focus the stethoscope's acoustic sensitivity on a smaller region within the heart from which murmur sounds originate. Beamforming, focusing, and steering of a detector's sensitivity requires multiple sensors, and its feasibility depends on the number, size, and physical arrangement of the sensors; as well as the frequency and location of the sound source, and the physical properties of the medium between the source and the sensors.

In the proposed multiple input stethoscope array, each stethoscope diaphragm is secured to a supportive apparatus so that the sensors contact all five landmarks when the fixture is secured to the patient's chest. Straps are used to secure the apparatus to the patient since manually holding the device corrupts the signal with noise.

After collecting the data, a cross correlation is performed over all possible pairs of input signals (32 total) to align heart sound features in each of the five signals. Cross correlation is a procedure whereby two signals, offset in time relative to each other, are multiplied together sample-by-sample and then summed. The process continues until both signals are correlated at every possible offset, where one signal is fixed in time while the other advances by a single sample per correlation. The five signals are then aligned where the correlation is the greatest, and summing the aligned signals averages out the noise and improves the PCG's SNR.

\subsection{Beamforming}

The acoustic pickup of a stethoscope receives sound waves incident from many directions. The elements of the stethoscope array are situated closest to the five listening locations, but nonetheless receive unwanted sounds from other areas of the body. The coherent averaging technique is an effective solution to reduce noise and enhance the quality of heart sounds in a PCG; however, data collection and processing occur independently — the sensors are only synchronized to start and stop together but otherwise 
operate independently until the data is collected. An improved method, namely, beam steering or beamforming, uses the relative locations of the five sensors and the wave speed to electronically "steer" the beam pattern towards the sound source.

A useful tool to understand wave reception is the beam pattern or directivity pattern. The directivity pattern is a spherical plot that displays a field's intensity as a function of incident angle. When sound propagates from multiple locations, the wave fronts produce a field pattern of peaks and troughs caused by constructive and destructive interference. Knowing this, a sensor array can alter its directivity pattern by applying a phase offset to each element to either constructively or destructively align the beam pattern in a particular direction. This allows heart signals originating from different directions to be collected and analyzed separately, rather than averaged together.

\subsection{Acoustic Aperture}

An aperture is a region in space that transmits or receives propagating waves [21, $\mathrm{p}$. 3]. A digital stethoscope is a passive aperture that only receives acoustic waves as opposed to an ultrasound machine, which is an array of active apertures that both sends and receives acoustic waves to image the internal organs [22]. Thus, only passive apertures are considered for this thesis. A propagating acoustic wave is described by its intensity, or sound pressure, $x(t, \mathbf{r})$ as a function of time and position; alternatively, the wave can be described by its Fourier transformed intensity $X(f, \mathbf{r})$ as a function of frequency and position. The aperture function, or sensitivity function, $A(f, \mathbf{r})$ relates the wave intensity incident on the aperture, $X(f, \mathbf{r})$, to the wave intensity received by the aperture, $X_{R}(f, \mathbf{r})[21$, p. 3]:

$$
X_{R}(f, \mathbf{r})=A(f, \mathbf{r}) X(f, \mathbf{r})
$$

where the position vector $\mathbf{r}=\left[\begin{array}{lll}x & y & z\end{array}\right]^{\mathrm{T}}$ is relative to the sound source location. 
The wave equation is a general formula that describes the propagation of acoustic and electromagnetic waves, among other types of waves [21, p. 2]:

$$
\nabla^{2} x(t, \mathbf{r})-\frac{1}{c^{2}} \frac{\delta^{2}}{\delta t^{2}} x(t, \mathbf{r})=0
$$

An acoustic wave resembles a spherical wave front in the near field, which is close to the origin of transmission $[21$, p. 6]. As the wave propagates into the far field, the profile flattens and approximates a planar wave front. The distinction between the near field and far field is dependent upon wavelength, aperture shape, and distance from the source to the aperture. The wave equation has both near field (planar) and far field (spherical) solutions [21, p. 2]:

$$
\begin{gathered}
x(t, \mathbf{r})=A e^{j(\omega t-\mathbf{k} \cdot \mathbf{r})}(\text { planar }) \\
x(t, \mathbf{r})=-\frac{A}{4 \pi r} e^{j(\omega t-\mathbf{k} \cdot \mathbf{r})}(\text { spherical })
\end{gathered}
$$

where the wavenumber:

$$
\mathbf{k}=\frac{2 \pi}{\lambda}\left[\begin{array}{lll}
\sin \theta \cos \phi & \sin \theta \sin \phi & \cos \theta
\end{array}\right]
$$

is a vector relative to the sound source location that is oriented along the direction of wave propagation and that measures the spatial wave density [21, p. 2]. The direction is specified as an angle, so each element in the vector is shown as a conversion from spherical to rectangular coordinates. Spherical coordinates are comprised of the $(r, \theta, \phi)$ dimensions, where $r$ is the magnitude, $\theta$ is the polar angle, and $\phi$ is the azimuthal angle, as shown in Figure 2-2. The phase shift given by the dot product $\mathbf{k} \cdot \mathbf{r}$ is maximized when the position vector is aligned with the direction of wave propagation. 


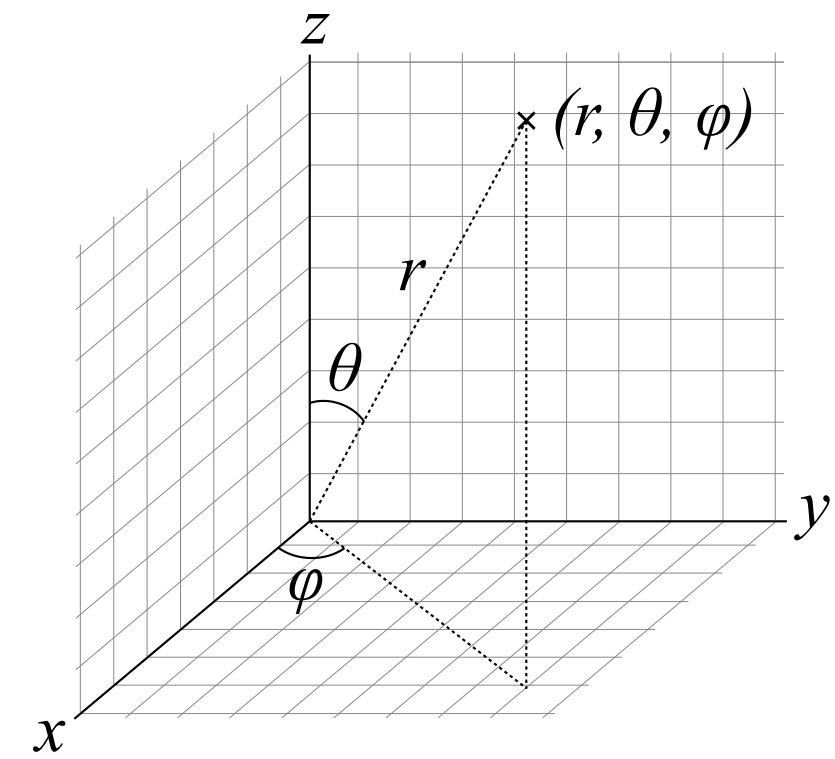

Figure 2-2: Spherical coordinate system [23].

\subsection{Directivity Pattern}

The aperture response $A(f, \mathbf{r})$ is a function of frequency and position. Position, however, is not a convenient reference because there is no way to directly compare apertures of different sizes and shapes, so an aperture response that depends on frequency and direction of arrival $(D O A)$ is preferred. The Fourier transform is able to convert the aperture response from the spatial domain to the angular domain and replace the position vector with the wavenumber. In signal analysis, the Fourier transform is typically used to transform signals between the time and frequency domain but, in general, can transform signals between any two domains. The directivity pattern, or beam pattern, $D(f, \boldsymbol{\alpha})$ is the Fourier-transformed aperture response that is dependent on DOA instead of position [21, p. 4]:

$$
\begin{gathered}
\boldsymbol{\alpha}=\frac{\mathbf{k}}{2 \pi}=\frac{1}{\lambda}\left[\begin{array}{ccc}
\sin \theta \cos \phi & \sin \theta \sin \phi \quad \cos \theta
\end{array}\right] \\
D(f, \boldsymbol{\alpha}) \Leftrightarrow A(f, \mathbf{r}) \\
D(f, \boldsymbol{\alpha})=\int_{-\infty}^{\infty} A(f, \mathbf{r}) e^{-j 2 \pi \boldsymbol{\alpha} \cdot \mathbf{r}} d \mathbf{r}
\end{gathered}
$$


In practice, the directivity pattern is presented as a two dimensional slice of a three dimensional pattern, where the frequency and one angle are held constant to demonstrate how the sensitivity varies over the other angle.

\subsection{Aperture Array}

The directivity pattern is not limited to a single continuous aperture, as the analysis can be extended to microphone arrays as well. Since the linearity property states that the Fourier transform of a sum of scaled responses is the sum of the scaled Fourier transforms of the individual responses, the total response of an array is the superposition of the individual responses [24, p. 97]:

$$
\mathbb{F}\left\{\sum_{n=1}^{N} K_{n} A_{n}\left(f, \mathbf{r}_{n}\right)\right\}=\sum_{n=1}^{N} K_{n} \mathbb{F}\left\{A_{n}\left(f, \mathbf{r}_{n}\right)\right\}
$$

where $K_{n}$ is the constant scaling factor for each aperture and $N$ is the total number of apertures. The array can be modeled as a sampled continuous aperture, where each microphone is an ideal point aperture. The sampling process consists of multiplying each aperture response with a Dirac delta impulse function. This approach is similar to the Discrete Time Fourier Transform (DTFT) [24, p. 101], with the exception that the sampling period (distance) is not necessarily constant. The delta function samples the sensitivity function by decomposing the continuous aperture into a finite collection of point apertures through the product property of the impulse [25, p. 24]:

$$
A(\mathbf{r}) \delta\left(\mathbf{r}-\mathbf{r}_{0}\right)=A\left(\mathbf{r}_{0}\right) \delta\left(\mathbf{r}-\mathbf{r}_{0}\right)
$$

and the array's aperture function is:

$$
A_{\text {array }}\left(f, \mathbf{r}_{n}\right)=\sum_{n=1}^{N} A_{n}\left(f, \mathbf{r}_{n}\right) \delta\left(\mathbf{r}-\mathbf{r}_{n}\right)
$$


The Fourier transform of a scaled, spatially offset impulse function applies a phase shift to each aperture but does not alter the magnitude response [24, p. 96]:

$$
A\left(\mathbf{r}_{0}\right) \delta\left(\mathbf{r}-\mathbf{r}_{0}\right) \Leftrightarrow A\left(\mathbf{r}_{0}\right) e^{-j 2 \pi \alpha \cdot \mathbf{r}_{0}}
$$

Thus, each sensitivity function is treated as a constant in the Fourier transformed response, so the array's directivity pattern is $[21$, p. 8]:

$$
D_{\text {array }}(f, \boldsymbol{\alpha})=\sum_{n=1}^{N} A_{n}(f) e^{-j 2 \pi \boldsymbol{\alpha} \cdot \mathbf{r}_{n}}
$$

\subsection{Beamforming}

The primary advantage of a microphone array, as opposed to a single aperture, is the ability to electronically steer the directivity pattern. This is accomplished by applying a complex exponential weight to each microphone [21, p. 19]:

$$
w_{n}(f)=a_{n}(f) e^{j \varphi_{n}(f)}
$$

where the amplitude $a_{n}(f)$ alters the shape of the directivity pattern, and the phase $\varphi_{n}(f)$ shifts or steers the pattern [21, p. 19]. This is because the inverse Fourier transform of a complex exponential produces a time delay, which phase aligns the wave fronts arriving at different elements in the array. Beamforming is the process of determining the weights in order to steer and focus the beam pattern towards the sound source for maximum reception [21, p. 19]. The simplest beamforming method is filter-sum beamforming, which applies a frequency dependent magnitude and phase weight to each element in the array [21, p. 23]:

$$
D_{\text {array }}(f, \boldsymbol{\alpha})=\sum_{n=1}^{N} w_{n}(f) A_{n}(f) e^{-j 2 \pi \boldsymbol{\alpha} \cdot \mathbf{r}_{n}}
$$




$$
=\sum_{n=1}^{N} a_{n}(f) A_{n}(f) e^{-j 2 \pi \boldsymbol{\alpha} \cdot \mathbf{r}_{n}} e^{j \varphi_{n}(f)}
$$

Delay-sum beamforming is a variation of filter-sum beamforming that applies a frequency dependent phase weight to steer the main lobe and a frequency independent, constant amplitude weight to normalize the maximum intensity [21, p. 22]:

$$
\begin{gathered}
a_{n}(f)=\frac{1}{N}, \varphi_{n}(f)=2 \pi \boldsymbol{\alpha}^{\prime} \cdot \mathbf{r}_{n} \\
D_{\text {array }}^{\prime}(f, \boldsymbol{\alpha})=\sum_{n=1}^{N} \frac{1}{N} A_{n}(f) e^{-j 2 \pi \boldsymbol{\alpha} \cdot \mathbf{r}_{n}} e^{j 2 \pi \boldsymbol{\alpha}^{\prime} \cdot \mathbf{r}_{n}} \\
=\frac{1}{N} \sum_{n=1}^{N} A_{n}(f) e^{-j 2 \pi\left(\boldsymbol{\alpha}-\boldsymbol{\alpha}^{\prime}\right) \cdot \mathbf{r}_{n}}
\end{gathered}
$$

The normalized wavenumber $\boldsymbol{\alpha}$ depends on both the DOA and wavelength. In practice, the speed of sound through human tissue and the frequency of interest are known while the wavelength is not, so it is convenient to replace wavelength with both wave speed and frequency using the relationship:

$$
\lambda f=v \rightarrow \lambda=\frac{v}{f}
$$

Furthermore, the normalized wavenumber can be expressed as the direction vector $\boldsymbol{\beta}$ :

$$
\boldsymbol{\alpha}=\frac{\boldsymbol{\beta}}{\lambda} \rightarrow \boldsymbol{\beta}=\left[\begin{array}{lll}
\sin \theta \cos \phi & \sin \theta \sin \phi \quad \cos \theta
\end{array}\right]
$$

Thus, the final beam steering formula separates environmental assumptions (frequency and velocity) from the desired beam direction $(\boldsymbol{\beta} \propto \theta, \phi)[21$, p. 19]:

$$
D_{\text {array }}^{\prime}(f, \boldsymbol{\beta})=\frac{1}{N} \sum_{n=1}^{N} A_{n}(f) e^{-j \frac{2 \pi}{\lambda}\left(\boldsymbol{\beta}-\boldsymbol{\beta}^{\prime}\right) \cdot \mathbf{r}_{n}}=\frac{1}{N} \sum_{n=1}^{N} A_{n}(f) e^{-j \frac{2 \pi f}{v}\left(\boldsymbol{\beta}-\boldsymbol{\beta}^{\prime}\right) \cdot \mathbf{r}_{n}}
$$


The directivity pattern, being a Fourier-transformed function, is susceptible to aliasing. Spatial aliasing is avoided when the spacing between any two sensors is less than half the wavelength. When the sensor spacing exceeds this limit, directionality is lost because the directivity pattern's main lobe is replicated in the side lobes [21, pp. 13-14].

\subsection{Simulation Results}

The five element stethoscope array constructed in [20] is simulated in MATLAB with the stethoscope positions in Figure 2-3.

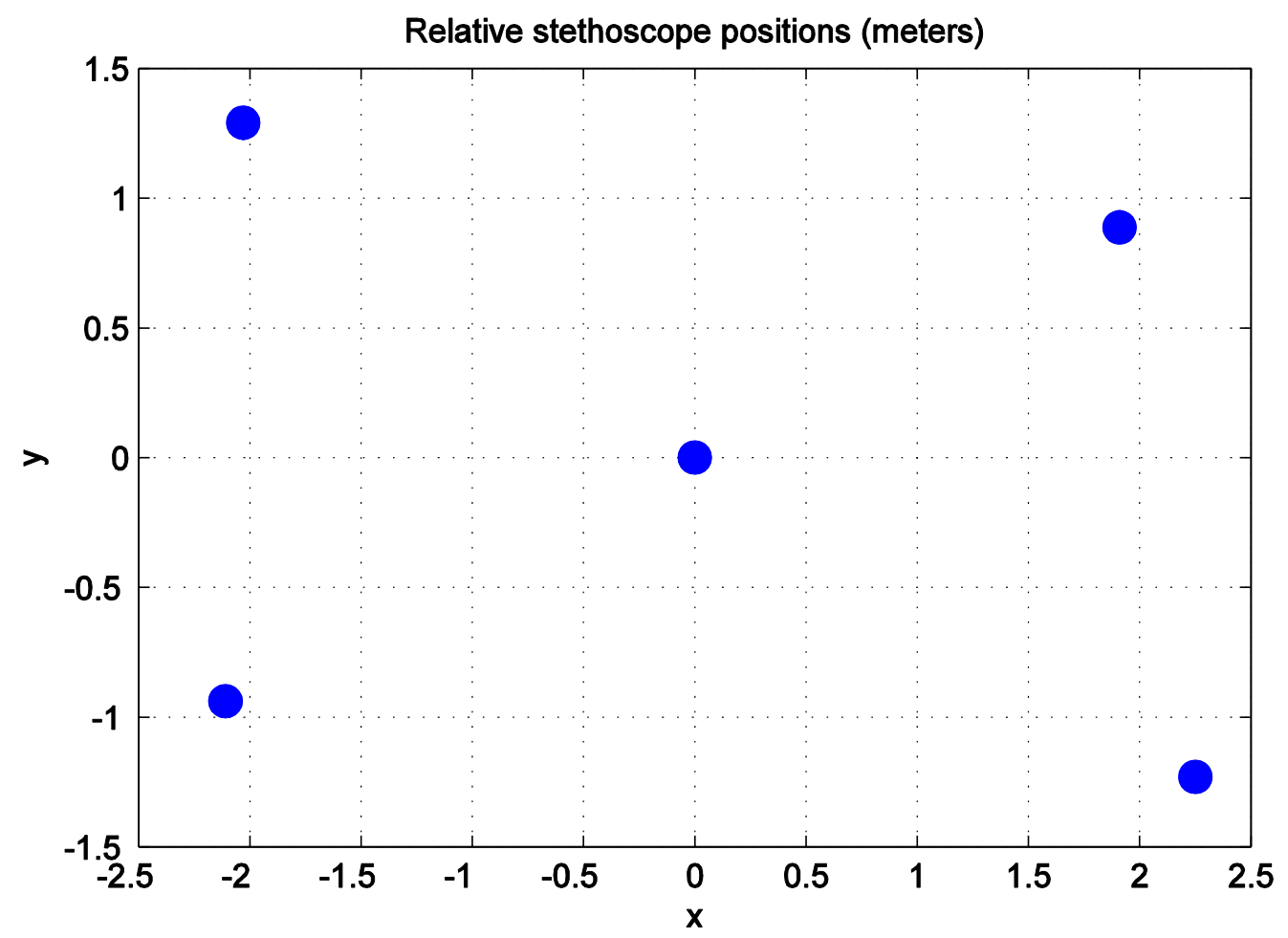

Figure 2-3: Stethoscope positions in the apparatus [beamforming.m].

The stethoscopes are situated so that they lay over the precordial landmarks when the apparatus is strapped onto the patient's chest. The stethoscopes comfortably conform to the chest since each one is placed in a PVC pipe with foam backing. This arrangement creates a slight z-plane offset which is difficult to measure and varies between patients, so it is ignored in the simulation. 
The simulation is run with the script beamforming.m, where the wave velocity is set to 1,540 meters/sec, which is the average speed of sound through human tissue [26]. The target azimuthal angle $\phi$ iterates by $\frac{\pi}{6}$ radians through the range 0 to $\pi$, and the directivity pattern is computed for three different frequencies. 
The first simulation is performed at a frequency of $500 \mathrm{~Hz}$, which is within the typical murmur range of 125 to $800 \mathrm{~Hz}$ but less than the threshold for spatial aliasing. The results are displayed in Figure 2-4.
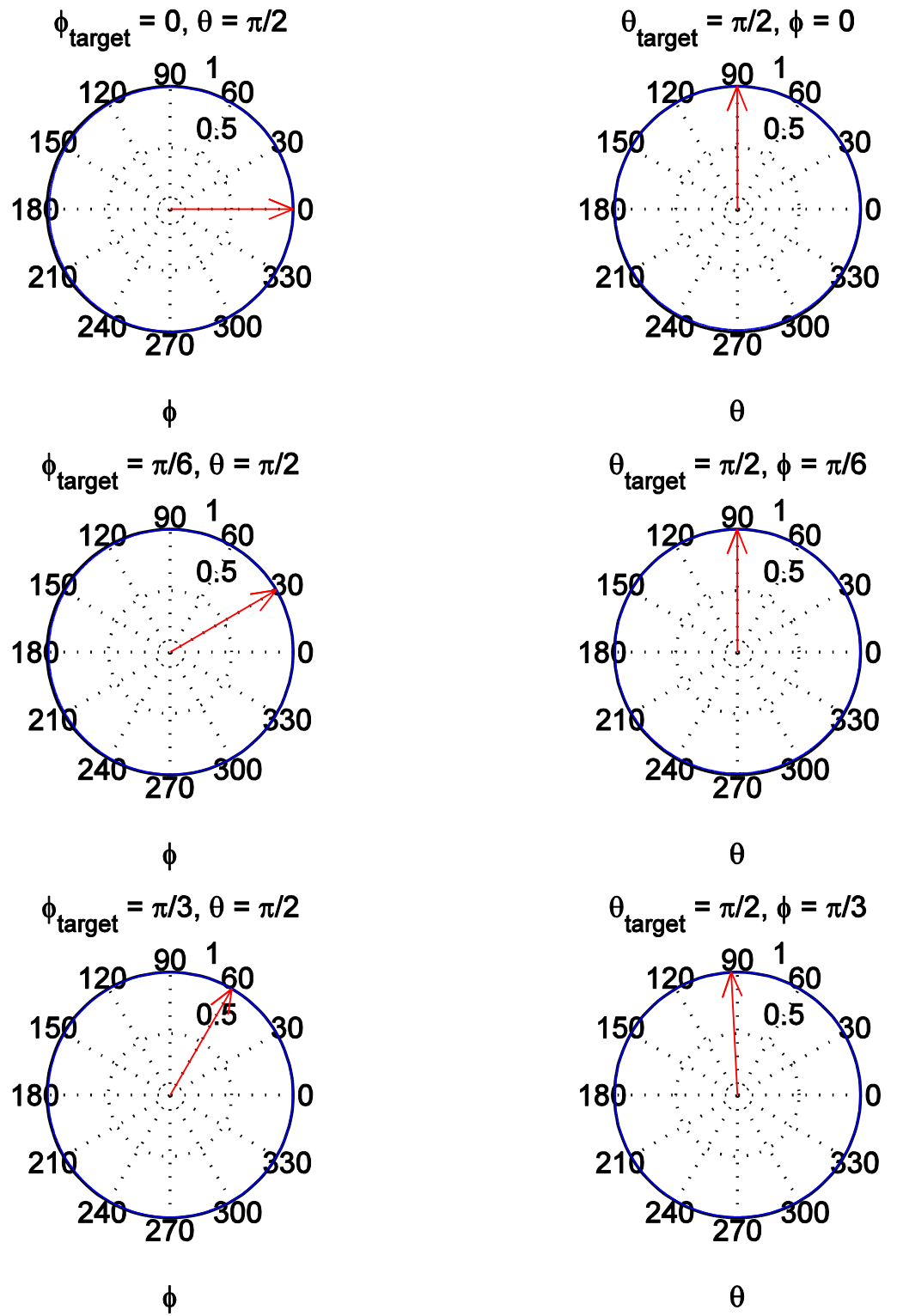

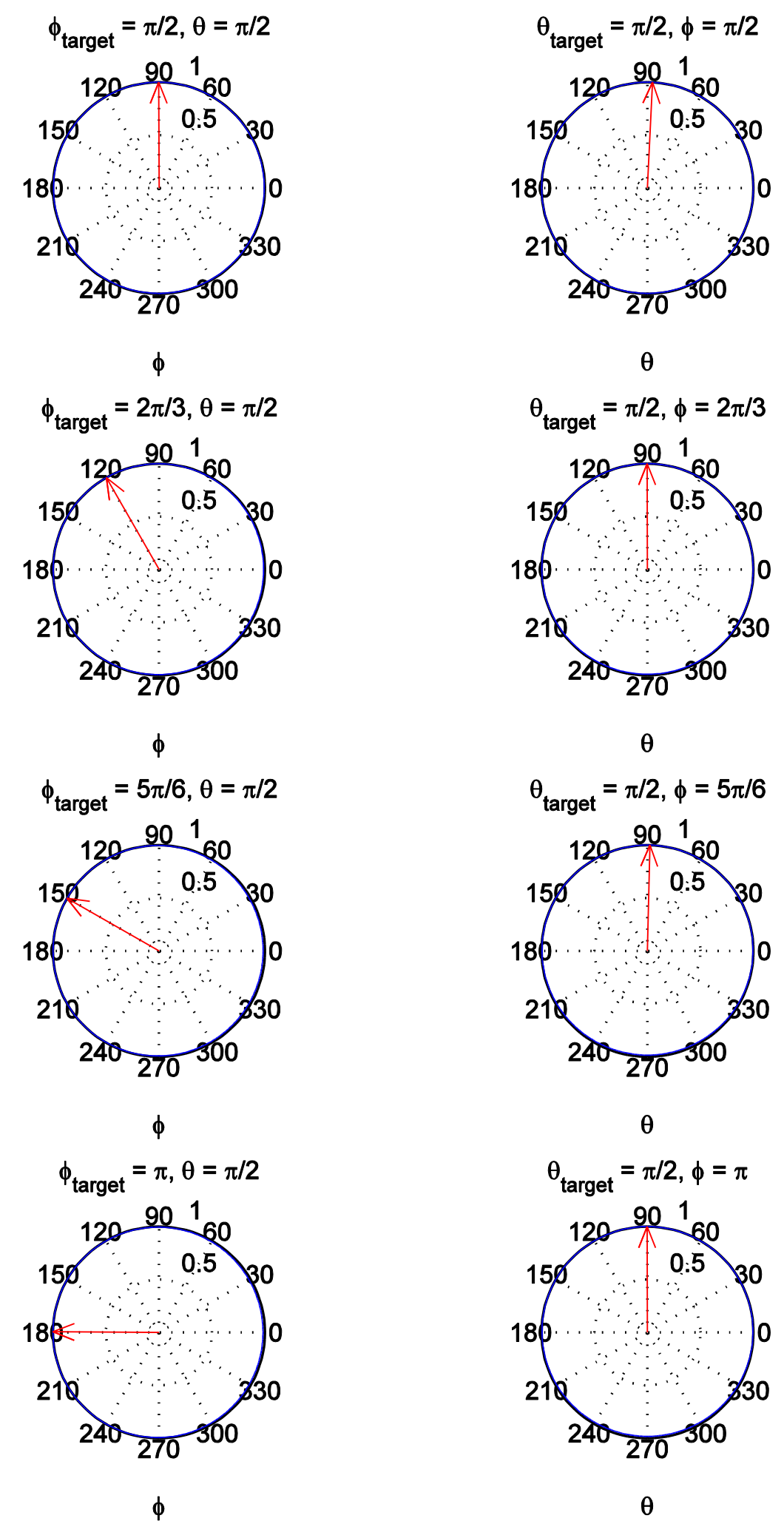

Figure 2-4: Steering $\Phi$ between 0 and $\pi(f=500 \mathrm{~Hz}$, no spatial aliasing). 
The second simulation is performed at a frequency of $7 \mathrm{kHz}$, which is greater than the typical murmur range but still less than the threshold for spatial aliasing. The results are displayed in Figure 2-5.
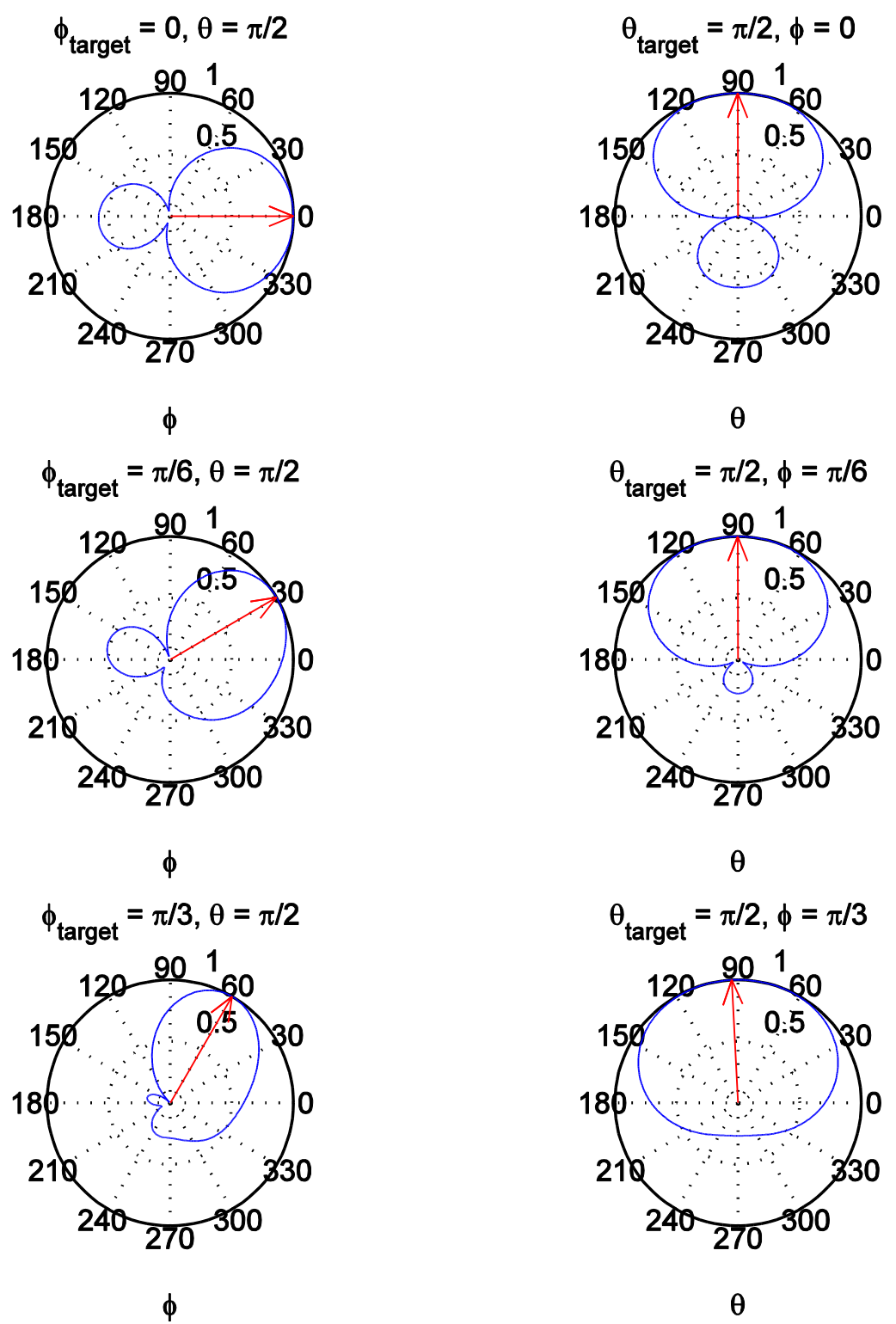

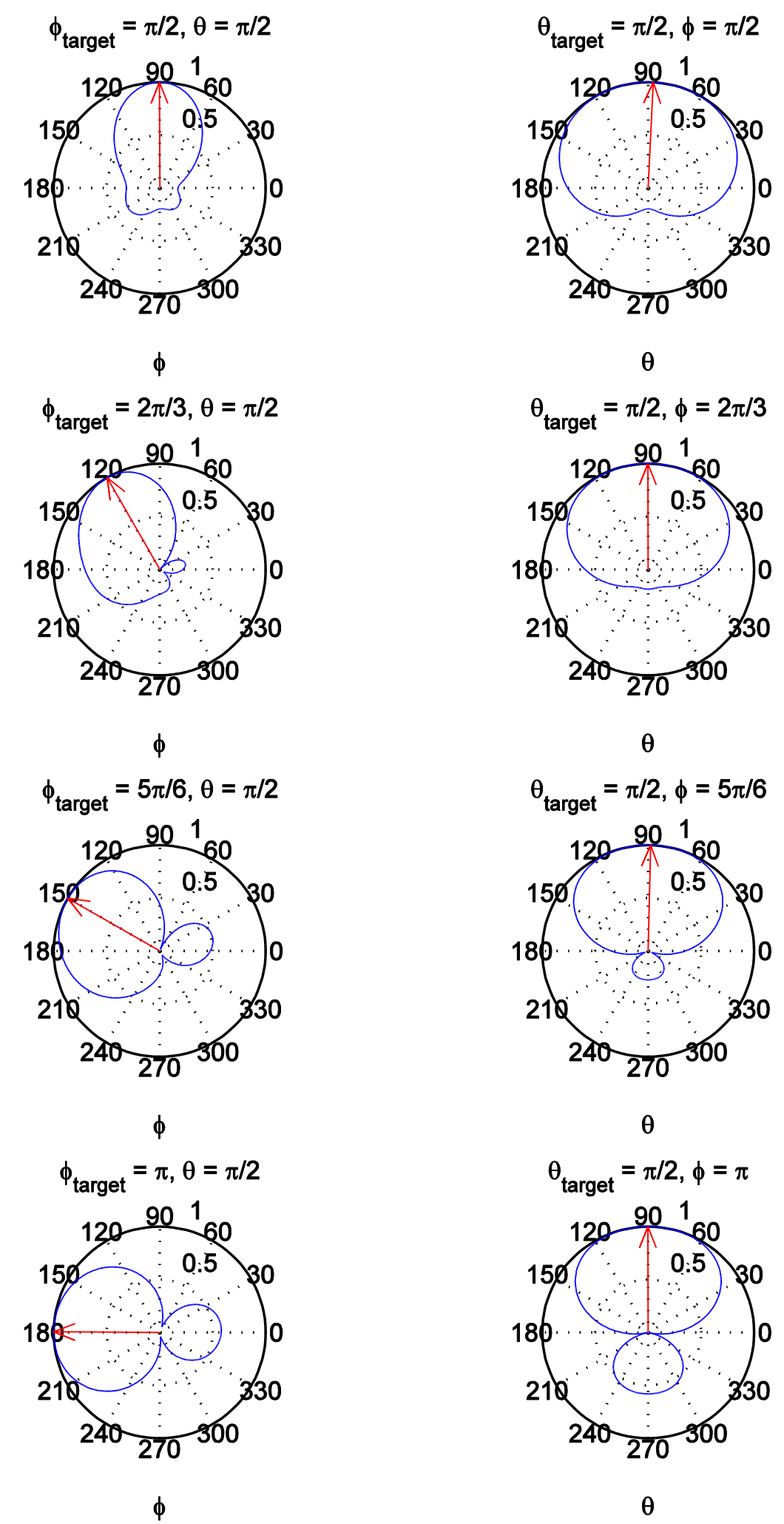

Figure 2-5: Steering $\Phi$ between 0 and $\pi$ ( $f=7 \mathrm{kHz}$, no spatial aliasing). 
The third simulation is performed at a frequency of $20 \mathrm{kHz}$, which is inaudible, greater than the murmur range, and greater than the threshold for spatial aliasing. The results are displayed in Figure 2-6.
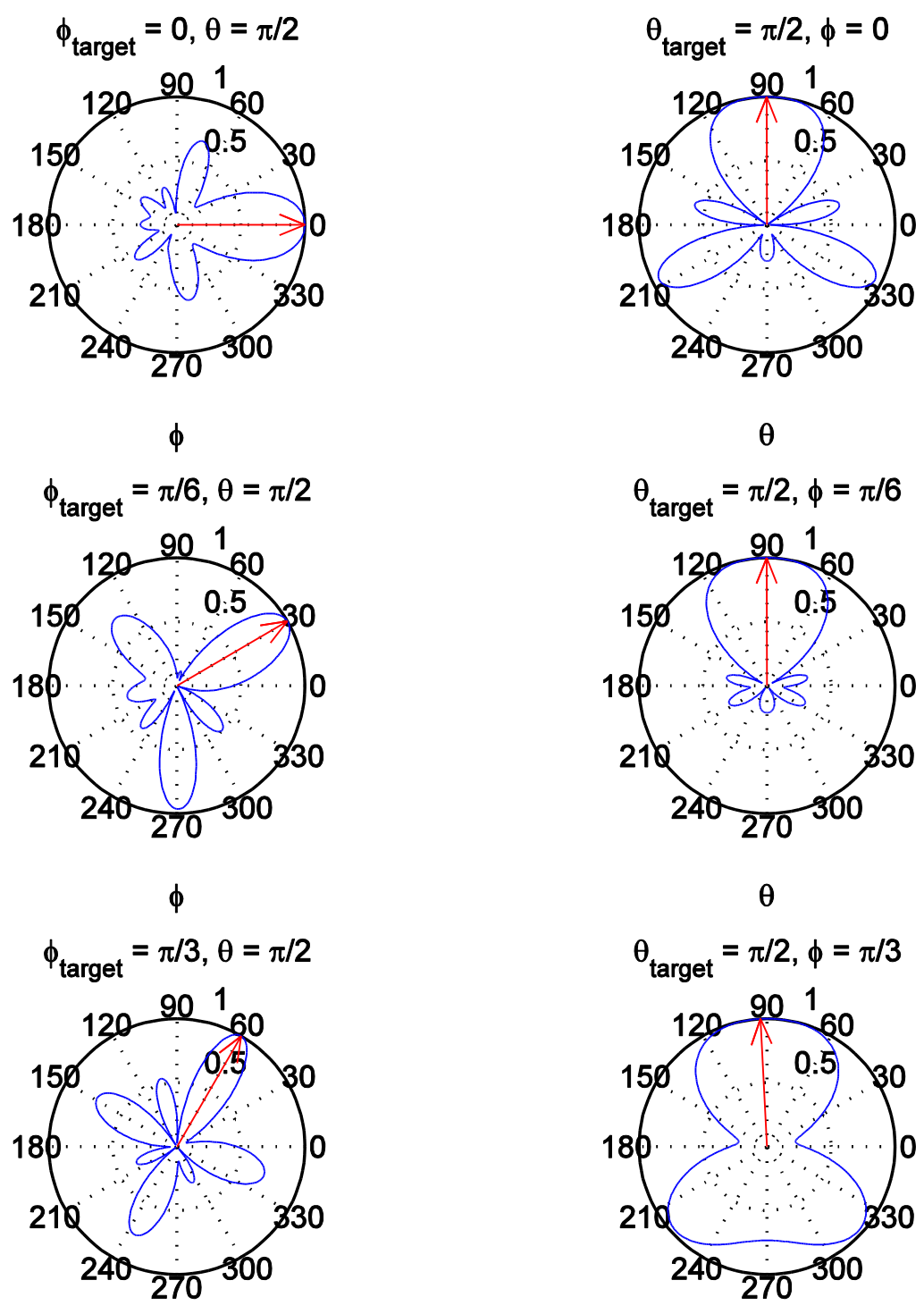

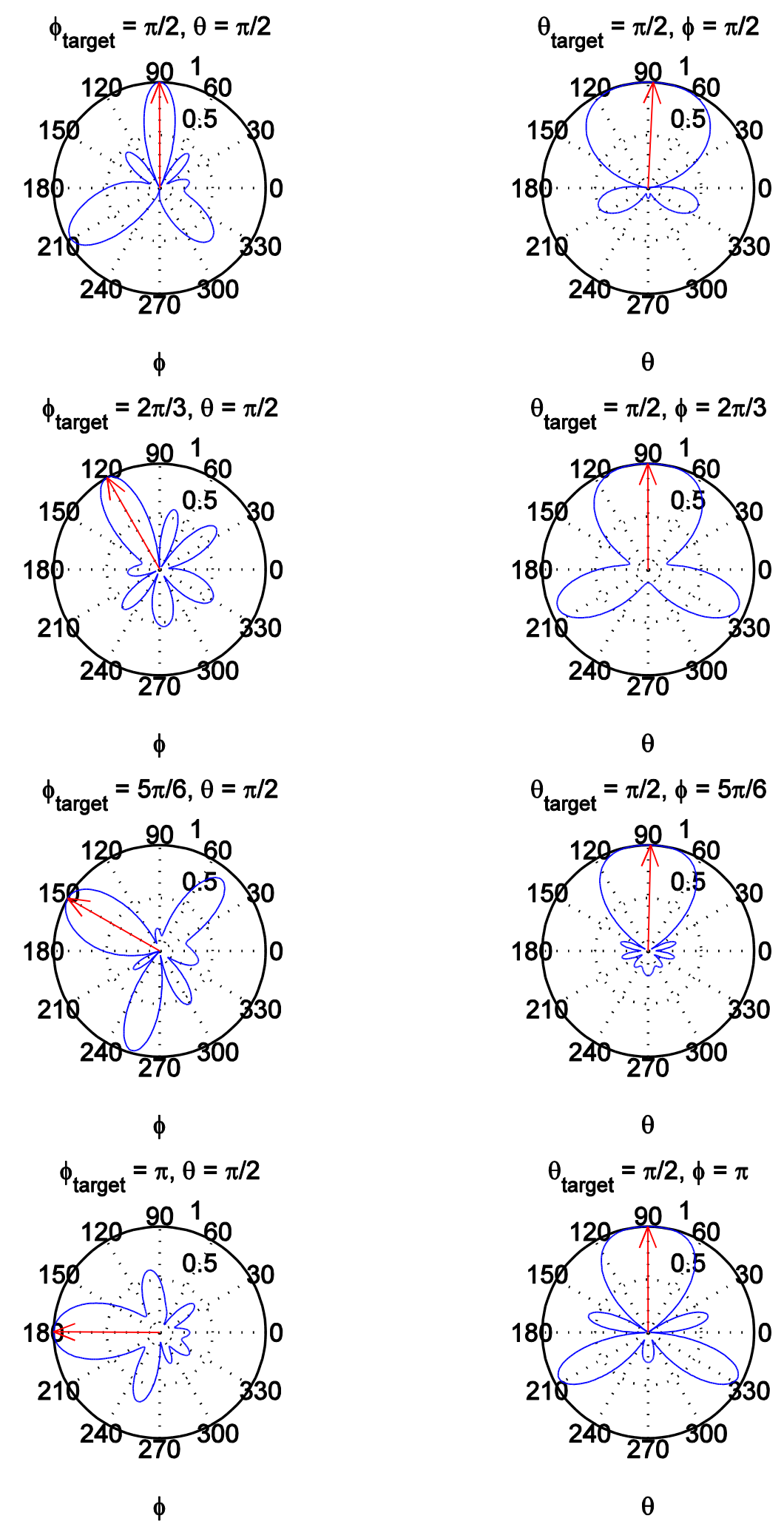

Figure 2-6: Steering $\Phi$ between 0 and $\pi(f=20 \mathrm{kHz}$, spatial aliasing). 


\subsection{Discussion}

The simulation at $7 \mathrm{kHz}$ is the most effective of the three frequencies for beamforming applications. The main lobe (red arrow) is sufficiently narrow and effectively tracks the target angle, and the wavelength is large enough compared to the sensor spacing to prevent spatial aliasing. Unfortunately, the frequency is too large for the murmur spectral range.

The simulation at $20 \mathrm{kHz}$ has the narrowest main lobe at the expense of spatial aliasing, which duplicates the main lobe at the side lobes. The main lobe nonetheless accurately tracks the target angle, but like the $7 \mathrm{kHz}$ simulation, the frequency range exceeds that of the murmur, and is therefore unsuitable for the stethoscope array.

The simulation at $500 \mathrm{~Hz}$ has the least directionality of the three simulations. The directivity pattern is roughly a sphere without a distinguishable main lobe. The maximum intensity (red arrow) is only slightly greater than the rest of the pattern's intensity, but it does manage to track the target angle nonetheless. Additionally, the wavelength is large enough compared to the sensor spacing that spatial aliasing does not exist. Unfortunately, the uniformity of the directivity pattern makes this frequency unsuitable for effective beam steering.

These three simulations show that the main lobe's width is inversely proportional to the frequency. However, it is also known that sensor density is inversely proportional to the main lobe width. Adding more stethoscopes to the array could possibly make beamforming feasible in the murmur's frequency range, but the size and constrained locations of the stethoscopes makes it impractical to add more. Therefore, beamforming is not a suitable technique for the five element stethoscope array since the frequencies in the heart murmur range do not produce a narrow enough main lobe to steer the beam pattern. 


\section{Segmentation Algorithms and Concepts}

\subsection{Frequency Domain Filtering}

The Fourier series maps a periodic, continuous signal to the discrete Fourier coefficients $c_{k}$ located at integer multiples of the fundamental frequency $\omega_{0}[24$, p. 84]:

$$
c_{k}=\frac{1}{T_{0}} \int_{T_{0}} x(t) e^{-j k \omega_{0} t} d t
$$

The Fourier transform $\mathbb{F}$ maps an aperiodic, continuous signal to the continuous frequency spectrum $X(\omega)$ by evaluating the limit of the Fourier series coefficients as the period approaches infinity [24, p. 91]:

$$
\begin{gathered}
\lim _{T_{0} \rightarrow \infty} \frac{2 \pi}{T_{0}}=d \omega \stackrel{\omega_{0}=\frac{2 \pi}{T_{0}}}{\longrightarrow} \lim _{T_{0} \rightarrow \infty} k \omega_{0}=k d \omega=\omega \\
c_{k \infty}=\lim _{T_{0} \rightarrow \infty} \frac{1}{2 \pi} \frac{2 \pi}{T_{0}} \int_{T_{0}} x(t) e^{-j k \omega_{0} t} d t=\frac{1}{2 \pi}\left[\int_{-\infty}^{\infty} x(t) e^{-j \omega t} d t\right] d \omega=\frac{1}{2 \pi} X(\omega) d \omega \\
\mathbb{F}\{x(t)\} \equiv X(\omega)=\int_{-\infty}^{\infty} x(t) e^{-j \omega t} d t
\end{gathered}
$$

The shared connection between the Fourier series and the Fourier transform is that both use a periodic basis function to quantify the frequency distribution. In practice, it is simpler to use the Fourier transform for both periodic and aperiodic signals. Instead of integrating for the Fourier series, periodic signals can be truncated to a single period and transformed using tables of common transform pairs and properties. Thus, the concepts presented here apply equally to the Fourier transform and the Fourier series. 
The utility of the complex exponential basis function is obscured by its notation. Euler's identity reveals that the complex exponential is fundamentally sinusoidal [24, $\mathrm{p}$. 84]:

$$
e^{-j \omega t}=\cos (\omega t)-j \sin (\omega t)
$$

The symbol $j$ denotes that the real and imaginary components are separate stores of information. In fact, sine and cosine are orthogonal functions [24, p. 83]:

$$
\int_{0}^{2 \pi} \cos (\omega t) \sin (\omega t) d t=0
$$

because they are completely uncorrelated, despite only differing by a phase offset. Since sine and cosine both exist in $\mathbb{R}^{2}$, and addition or subtraction would only superimpose the two sinusoids, they are instead represented as orthogonal vectors in the complex plane or $\mathbb{C}^{2}$. Thus, their vector sum is the complex exponential, so that the cosine component lies on the real axis, the sine component lies on the imaginary axis, and the locus of all points is the unit circle.

Applying Euler's identity to the complex exponential demonstrates that the Fourier transform is a correlation between the input signal and two orthogonal sinusoids [24, $\mathrm{p}$. 603]:

$$
X(\omega)=\int_{-\infty}^{\infty} x(t) \cos (\omega t) d t-j \int_{-\infty}^{\infty} x(t) \sin (\omega t) d t
$$

In general, both integrals are necessary for determining the spectrum of any continuous, physically realizable signal. For example, the sine and cosine Fourier transform pairs are given as [24, p. 96]:

$$
\cos \left(\omega_{0} t\right) \leftrightarrow \pi\left[\delta\left(\omega-\omega_{0}\right)+\delta\left(\omega+\omega_{0}\right)\right]
$$




$$
\sin \left(\omega_{0} t\right) \leftrightarrow-j \pi\left[\delta\left(\omega-\omega_{0}\right)-\delta\left(\omega+\omega_{0}\right)\right]
$$

where the Dirac delta function, $\delta\left(\omega-\omega_{0}\right)$, is an infinite-magnitude, infinitesimal duration pulse located at the sinusoid's frequency $\omega_{0}$. The cosine spectrum is purely real whereas the sine spectrum is purely imaginary, but a time delayed cosine has both real and imaginary spectral components [24, pp. 96-97]:

$$
\begin{gathered}
\cos \left(\omega_{0} t-t_{0}\right) \leftrightarrow \pi\left[\delta\left(\omega-\omega_{0}\right)+\delta\left(\omega+\omega_{0}\right)\right] e^{-j \omega t_{0}} \\
=\cos (\omega t)\left[\delta\left(\omega-\omega_{0}\right)+\delta\left(\omega+\omega_{0}\right)\right]-j \sin (\omega t)\left[\delta\left(\omega-\omega_{0}\right)+\delta\left(\omega+\omega_{0}\right)\right]
\end{gathered}
$$

Thus, the delayed cosine correlates with both sine and cosine in the time domain. Ultimately, there are some signals that only require one of the integrals in the Fourier transform, but in general, continuous signals require both the sine and cosine integrals.

Just as a spectrum can be obtained through Fourier analysis, a signal can be constructed from a spectrum. Any periodic signal can be represented with its Fourier series coefficients as [24, p. 84]:

$$
x(t)=\sum_{k=-\infty}^{\infty} c_{k} e^{j k \omega_{0} t}=c_{0}+\sum_{k=1}^{\infty} 2\left|c_{k}\right| \cos \left(k \omega_{0} t+\theta_{k}\right)
$$

The last expression is the combined trigonometric form or polar form of the Fourier series, and it demonstrates how a periodic signal can be theoretically generated from a superposition of amplitude weighted and phase shifted sinusoids. This principle can be extended to aperiodic signals through the inverse Fourier transform $\mathbb{F}^{-1}[24$, p. 91]:

$$
\begin{gathered}
c_{k \infty}=\frac{1}{2 \pi} X(\omega) d \omega \\
x(t)=\sum_{k=-\infty}^{\infty} c_{k \infty} e^{j k \omega_{0} t}=\sum_{k=-\infty}^{\infty}\left[\frac{1}{2 \pi} X(\omega) d \omega\right] e^{j k \omega_{0} t}=\frac{1}{2 \pi} \sum_{k=-\infty}^{\infty} X(\omega) e^{j k \omega_{0} t} d \omega
\end{gathered}
$$




$$
\mathbb{F}^{-1}\{X(\omega)\} \equiv x(t)=\frac{1}{2 \pi} \int_{-\infty}^{\infty} X(\omega) e^{j \omega t} d \omega
$$

Knowing this, it is possible to attenuate undesirable bands of frequencies, such as murmurs, and then reconstruct a new signal. However, this is only possible when the heart sound and murmur frequencies do not overlap in the spectrum, but this is not always the case as can be seen in Figure 3-1. Since the Fourier transform uses a periodic basis function, it is assumed that the frequencies exist for all time, but PCG's are non-stationary signals because the frequency content varies over time.

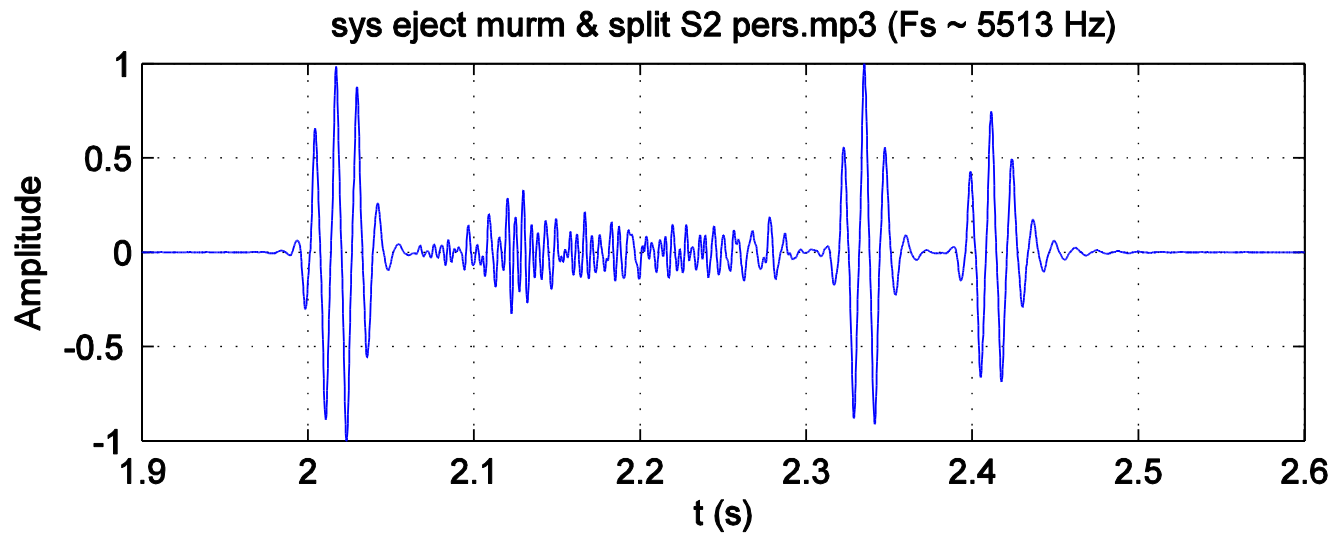

FFT - Magnitude Response

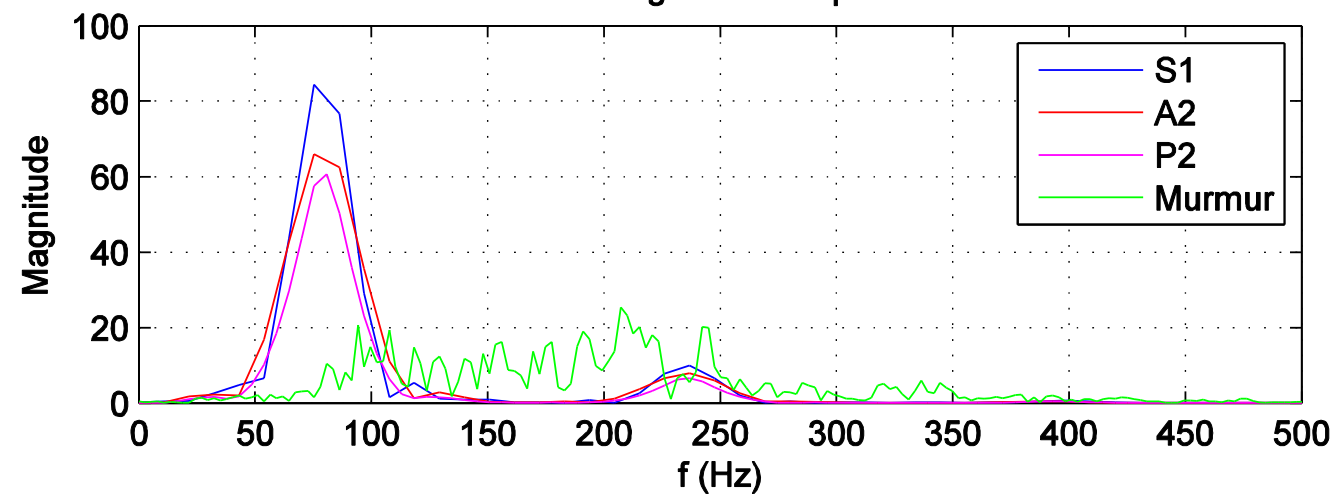

Figure 3-1: PCG spectrum [PCG_FFT.m].

The Short-Term Fourier Transform (STFT) is a proposed solution because it adds time resolution to the spectrum by dividing the signal into segments and performing a separate Fourier transform on each segment [24, p. 606]: 


$$
\operatorname{STFT}(\tau, \omega)=X_{S}(\omega)=\int_{t}[x(t) w(t-\tau)] e^{-j \omega t} d t
$$

As a result, the STFT replaces the spectrum with a spectrogram, which has both time and frequency axes and either a third axis or color scheme representing the magnitude. However, the shortcoming of the STFT is that even though a greater number of windows increases resolution in the time domain, resolution in the frequency domain diminishes. Therefore, another technique is required for locating frequencies in time.

\subsection{Wavelet Transform}

\subsubsection{Continuous Wavelet Transform}

The Fourier transform is incapable of locating frequencies in the time domain because the complex exponential is a periodic basis function of infinite extent, whereas the signal itself is of finite extent. Instead of dividing the signal into segments and performing separate Fourier transforms on each segment (STFT), the wavelet transform replaces the periodic complex exponential basis function with an aperiodic, finite-duration wavelet. Since the wavelet function is localized in time, it is both scaled and shifted in the wavelet transform correlation to determine where the signal most closely matches the wavelet. Thus, the wavelet transform is a function of time and scale instead of frequency, so it excels at locating events in the time domain instead of determining the frequency content.

The continuous wavelet transform (CWT) is given by the integral [24, p. 611]:

$$
W(a, b)=\frac{1}{\sqrt{a}} \int_{-\infty}^{\infty} x(t) \psi^{*}\left(\frac{t-b}{a}\right) d t
$$

where $\psi$ is the mother wavelet, $a$ is the scaling parameter, and $b$ is the translation parameter. The mother wavelet is the aperiodic basis function that is cross correlated with the signal at different scale values. Alternatively, the scaling function $\varphi$ is a complementary 
function that can be substituted for the mother wavelet in the integral [24, p. 611]. In fact, the mother wavelet and the scaling function are equivalent to high-pass and low-pass filters.

The wavelet transform is typically applied to discrete time signals, which limits the translation parameter to integer values but does not restrict the scaling parameter. Therefore, the continuous wavelet transform is so named because the scaling parameter is continuous, even for discrete time inputs [24, p. 606].

\subsubsection{Discrete Wavelet Transform}

In contrast to the CWT, the discrete wavelet transform (DWT) does away with the scaling, translation, and correlation operations and replaces them with the equivalent, but more efficient, dyadic down sampling and convolution. The mother wavelet and scaling functions are the same functions from before, except that they are now treated as impulse responses that are convolved with the signal rather than correlated. As a result, the mother wavelet is the high pass decomposition filter, and the scaling function is the low pass decomposition filter.

The high pass decomposition filter is used to transform the input into the detail coefficients, while the low pass decomposition is used to transform the input into the approximation coefficients. The approximation and detail coefficients demonstrate how the time domain features change at different frequency bands or decomposition levels. The coefficients for the first level are acquired by separately convolving the PCG with the low pass and high pass decomposition filters and then down sampling each result by a factor of two to generate the approximation coefficient $\mathrm{CA}_{1}$ (low pass) and the detail coefficient $C D_{1}$ (high pass). Down sampling restricts the first level's frequency range to half the sampling rate of the PCG. In particular, $\mathrm{CA}_{1}$ represents the lower half of this frequency range and $C D_{1}$ represents the upper half of this frequency range. Each 
additional level is decomposed by repeating this procedure on the current level's approximation coefficient so that the final set of coefficients for $N$ levels, ordered from the lowest to highest frequency range, is $C A_{N}$ followed by $C D_{N}$ through $C D_{1}$. The approximation and detail coefficients can then be modified and reconstructed with the high pass and low pass reconstruction filters, and the two reconstructed waveforms are combined to generate the filtered output signal.

For heart sound segmentation, the DWT is used to attenuate murmurs so that the heart sounds can be segmented. This is achieved by determining the appropriate level (frequency band) where the heart sounds are concentrated, and then applying the low pass decomposition filter at this level to generate its approximation coefficient. Reconstructing just the approximation coefficient will generate an output waveform with attenuated murmurs. After the heart sounds are segmented, the original PCG is used to segment and classify the murmurs.

\subsection{Simplicity Transform}

Given that the discrete wavelet transform's purpose is to attenuate the murmurs without otherwise altering the PCG, the heart sounds and murmurs require separate waveforms for segmentation. Therefore, an alternative segmentation technique is proposed, one which transforms the PCG into a waveform that can be used to segment both the heart sounds and murmurs. The underlying algorithm is known as the simplicity transform because the PCG is transformed into a waveform where the values quantify the simplicity of short segments in the PCG.

\subsubsection{Complexity and Simplicity}

The Fourier transform produces its sparsest spectrum when the signal is either a constant or a sinusoid. The transform pair for the constant is: 


$$
A \leftrightarrow 2 \pi A \delta(\omega)
$$

Likewise, the transform of a sinusoid is a pair of delta functions (from before). Thus, these are the "simplest" signals because the spectrum only contains a single frequency. Alternatively, the Fourier transform of the rectangle function is a sine cardinal or sinc function (Figure 3-2):

$$
\operatorname{rect}(\mathrm{t}) \Leftrightarrow \operatorname{sinc}(f)=\frac{\sin (f)}{f}
$$

This is the most "complex" signal because its spectrum spans all frequencies. Consequently, sinusoids and rectangles are not physically realizable because real signals can neither persist for all time nor span all frequencies. However, both of these limiting cases demonstrate that "simple" signals have compact spectral ranges while "complex" signals have broad spectral ranges.
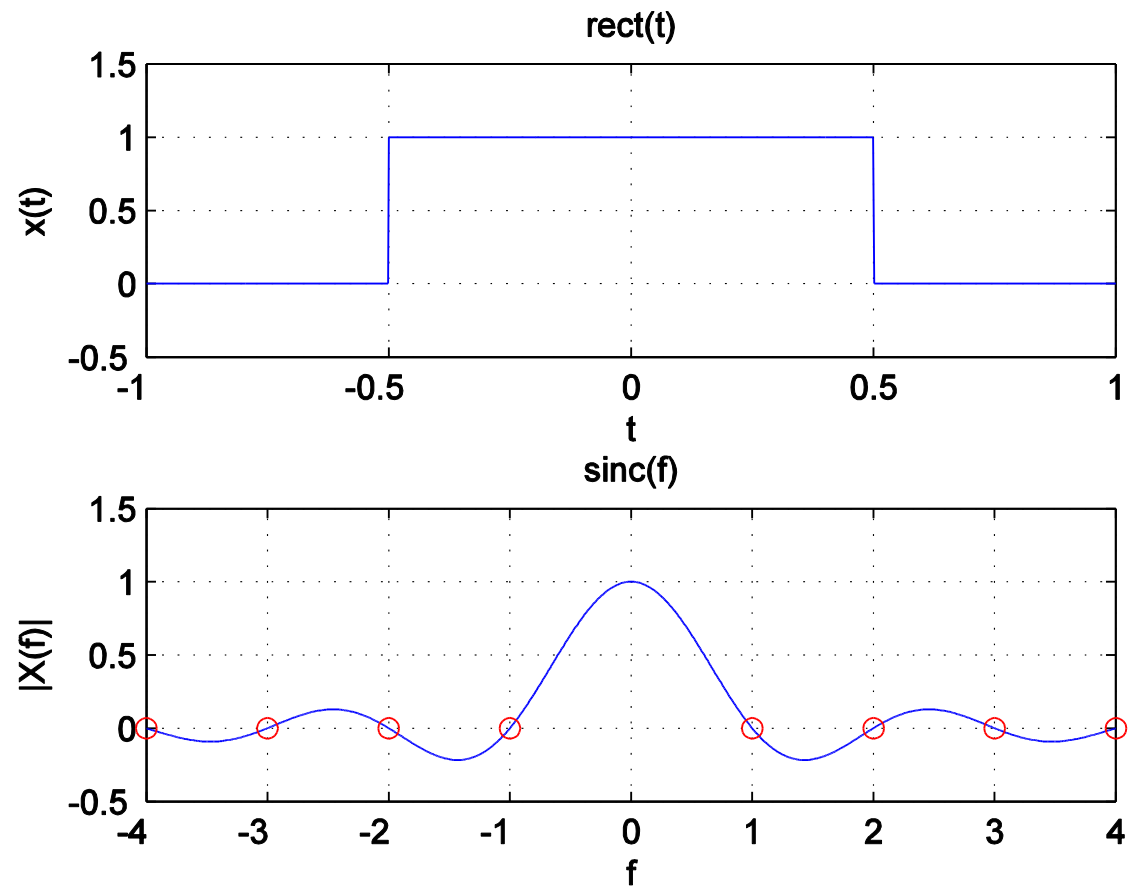

Figure 3-2: Fourier transform of the sinc function [rect_sinc.m]. 
Compared to the irregular shape of murmurs and background noise, heart sound segments (S1/S2 or S3/S4) resemble simple wavelet packets. Furthermore, auscultation reveals that murmurs resemble rumbles, clicks, or snaps while heart sounds are simple beats. Intuitively, heart sounds are "simple" while murmurs and noise are "complex". Thus, one way to separate heart sound segments from murmur segments is to calculate the signal's simplicity. Since heart sounds are the "simplest" segments in a PCG, the segments with simplicities greater than a threshold are classified as heart sounds, while the segments with simplicities less than the threshold are classified as either murmurs or noise. The inverse of simplicity is the complexity, so depending on the context, either term may be used to describe a signal. In general, Fourier analysis is unsuitable for quantifying simplicity, so the preferred method for calculating the simplicity is singular spectrum analysis.

\subsubsection{Dynamical Systems}

Dynamical systems theory is the study of how a system's state changes over time. The state is represented with state variables, which are elements of the $N$-dimensional state vector.

$$
\mathbf{y}(t)=\left[y_{1}, y_{2}, \ldots, y_{n-1}, y_{n}\right]^{T}
$$

The orbit or trajectory is the time evolution of the state vector in $\mathrm{N}$-dimensional state space or phase space. The dynamics of the system are the rules that specify a future state from an initial state, which are specified by the state transition function $\phi$ :

$$
\mathbf{y}(t)=\phi(t, \mathbf{y}(0))
$$

The initial state is time independent, so it can be placed at any position on the trajectory. Differentiating the state transition function produces a vector field in state space that assigns a "velocity" to every point on the trajectory [27]: 


$$
\frac{d \mathbf{y}(t)}{d t}=\mathbf{F}(\mathbf{y}(t))
$$

An illustrative state space example is the orbit of a planet around the sun. The state variables are the planet's position and velocity relative to the Earth, and the state transition function is Newton's Law of Gravitation. Prior to discovering solar system dynamics, namely, the heliocentric model, Kepler's elliptical orbit theory, and Newton's Laws, the planetary positions were geometrically tracked with epicycles [28]. Epicycles accurately predicted the locations of planets in the sky, but the state variables were hidden because it was assumed that the sun and the planets orbited Earth; and the state transition function was unknown because the Law of Gravitation was not yet discovered. Likewise, auscultation is used to accurately diagnose heart conditions without requiring complete knowledge of the heart's state or its underlying dynamics; but unlike epicycles, auscultation is still the most commonly used form of diagnosis because it is convenient, inexpensive, and nonintrusive.

\subsubsection{The Method of Delays}

In general, a measurement can be modeled as a function of a hidden state vector [13]:

$$
x(t)=h(\mathbf{y}(t))+w(t)
$$

Here the functional $h(\mathbf{y}(t))$ maps the state vector to a scalar, and $w(t)$ represents white noise. Although it is impossible to recover the state vector from a single measurement, Takens' embedding theorem states that it is possible to reconstruct the state space trajectory from a sufficient number of noiseless measurements. The $M$-dimensional delay vector [13, p. 1008]:

$$
\mathbf{x}_{i}(t)=[x(t), x(t-\tau), \cdots, x(t-(m-1) \tau)]^{T}(m>2 n+1)
$$


is an embedding, or one-to-one mapping, from $\mathrm{N}$-dimensional to $\mathrm{M}$-dimensional state space [29]. Takens' theorem proves that the delay vector and the state vector follow similar dynamics in different state spaces [13, p. 1008]:

$$
\mathbf{x}_{i}(t) \rightarrow \mathbf{x}_{i}(t+T) \Leftrightarrow \mathbf{y}(t) \rightarrow \mathbf{y}(t+T)
$$

Since a PCG (phonocardiogram) is a discrete time series, the delay $\tau$ is chosen to be the sampling period so that the delay vector simply contains consecutive samples.

In practice, Takens' theorem is impractical for reconstructing the signal's exact trajectory because measurements are always corrupted with noise. However, signal complexity is proportional to the dimension, rather than the trajectory, in state space, so estimating the dimension is sufficient. The "method of delays" is an extension of Takens' theorem for real signals, but instead of using a single delay vector to reconstruct the trajectory, it uses the complete set of delay vectors to estimate the dimension. The delay vector $\mathbf{x}_{i}(t)$ acts as a sliding window that is iteratively stored in a new row of the trajectory matrix $\mathbf{X}$ until the window reaches the end of the signal [13, pp. 1008-1009]:

$$
\begin{gathered}
\mathbf{X}=\frac{1}{\sqrt{P}}\left[\begin{array}{c}
\mathbf{x}_{1}^{T} \\
\mathbf{x}_{2}^{T} \\
\vdots \\
\mathbf{x}_{P}^{T}
\end{array}\right]=\frac{1}{\sqrt{P}}\left[\mathbf{x}_{I} \mathbf{x}_{I I} \cdots \mathbf{x}_{M}\right] \\
=\frac{1}{\sqrt{P}}\left[\begin{array}{cccc}
x(t) & x(t-\tau) & \cdots & x(t-(m-1) \tau) \\
x(t-\tau) & x(t-2 \tau) & \cdots & x(t-m \tau) \\
\vdots & \vdots & \ddots & \vdots \\
x(t-(p-1) \tau) & x(t-p \tau) & \cdots & x(t-(p-1) \tau-(m-1) \tau)
\end{array}\right]
\end{gathered}
$$

The trajectory matrix can be interpreted as either containing $P$ rows of $M$-dimensional delay vectors or $M$ columns of $P$-dimensional delay vectors. The subscripts represent the number of samples minus one that the delay vector is offset; in particular, the $M$ - 
dimensional delay vectors use Arabic numeral subscripts while the $P$-dimensional delay vectors use Roman numeral subscripts.

The trajectory matrix is the set of all delay vectors in the signal, so it is used to construct the correlation matrix [13, p. 1009], which quantifies the relationship between every pair of delay vectors:

$$
\begin{gathered}
\mathbf{C}=\mathbf{X}^{T} \mathbf{X}=\frac{1}{P}\left[\mathbf{x}_{1} \mathbf{x}_{2} \cdots \mathbf{x}_{P}\right]\left[\begin{array}{c}
\mathbf{x}_{1}^{T} \\
\mathbf{x}_{2}^{T} \\
\vdots \\
\mathbf{x}_{P}^{T}
\end{array}\right]=\frac{1}{P}\left[\begin{array}{c}
\mathbf{x}_{I}^{T} \\
\mathbf{x}_{I I}^{T} \\
\vdots \\
\mathbf{x}_{M}^{T}
\end{array}\right]\left[\mathbf{x}_{I} \mathbf{x}_{I I} \cdots \mathbf{x}_{M}\right] \\
=\left[\begin{array}{ccccc}
x(t) & x(t-\tau) & \cdots & x(t-(P-1) \tau) \\
x(t-\tau) & x(t-2 \tau) & \cdots & x(t-P \tau) \\
\vdots & \vdots & \ddots & \vdots & \\
x(t-(m-1) \tau) & x(t-m \tau) & \cdots & x(t-(P-1) \tau-(m-1) \tau)
\end{array}\right] \\
*\left[\begin{array}{ccccc}
x(t) & & x(t-\tau) & \cdots & x(t-(m-1) \tau) \\
x(t-\tau) & x(t-2 \tau) & \cdots & x(t-m \tau) \\
\vdots & & \vdots & \ddots & \vdots \\
x(t-(P-1) \tau) & x(t-P \tau) & \cdots & x(t-(P-1) \tau-(m-1) \tau)
\end{array}\right] \\
=\left[\begin{array}{cccc}
\mathbf{x}_{I}^{T} \mathbf{x}_{I} \quad \mathbf{x}_{I}^{T} \mathbf{x}_{I I} & \cdots & \mathbf{x}_{I}^{T} \mathbf{x}_{M} \\
\mathbf{x}_{I I}^{T} \mathbf{x}_{I} & \mathbf{x}_{I I}^{T} \mathbf{x}_{I I} & \cdots & \mathbf{x}_{I I}^{T} \mathbf{x}_{M} \\
\vdots & \vdots & \ddots & \vdots \\
\mathbf{x}_{M}^{T} \mathbf{x}_{I} & \mathbf{x}_{M}^{T} \mathbf{x}_{I I} & \cdots & \mathbf{x}_{M}^{T} \mathbf{x}_{M}
\end{array}\right] \Rightarrow C_{i j}=\mathbf{x}_{i}^{T} \mathbf{x}_{j}
\end{gathered}
$$

Each element in this matrix is a dot product of $P$-dimensional delay vectors instead of the original $M$-dimensional delay vectors because the inner dimension $M$ cancels during matrix multiplication. Thus, the correlation matrix completely characterizes the relationship between all pairs of $P$-dimensional delay vectors. A judicious selection of $M$ will provide a correlation matrix that is both computationally efficient and suitably descriptive enough to determine the complexity of the signal. 


\subsubsection{Eigenvalue Decomposition and the Singular Spectrum}

In order to determine system complexity, the correlation matrix must be decomposed into its eigenvalues. The eigenvalues and eigenvectors are given in the equation [13, $\mathrm{p}$. 1009]:

$$
\mathbf{C v}=\lambda \mathbf{v}
$$

where $\mathbf{v}$ is an eigenvector and $\lambda$ is an eigenvalue. The correlation matrix $\mathbf{C}$ represents a linear transformation, which can be visualized in three dimensional Euclidean space as a rotation, reflection, scaling, or shearing. Eigenvectors, by definition, are mutually orthogonal to each other and maintain the same orientation in space after the linear transformation. Thus, applying the transformation to an eigenvector is equivalent to scaling the eigenvector by its eigenvalue. The eigenvalues are found by rearranging the original equation into the characteristic equation and solving for the roots:

$$
\operatorname{det}(\mathbf{C}-\lambda \mathbf{v})=0
$$

where the maximum number of eigenvalues is the dimension $M$ of the correlation matrix. The singular spectrum is the set of eigenvalues stored in a diagonal matrix, ranked by magnitude in descending order:

$$
\lambda_{1}>\lambda_{2}>\cdots \lambda_{M} \Rightarrow \mathbf{D}=\left[\begin{array}{cccc}
\lambda_{1} & 0 & 0 & 0 \\
0 & \lambda_{2} & 0 & 0 \\
0 & 0 & \ddots & 0 \\
0 & 0 & 0 & \lambda_{M}
\end{array}\right]
$$

A system's complexity is quantified by the relative magnitude, and total number, of eigenvalues. A "simple" system contains only a few large-magnitude eigenvalues while a "complex" system contains many small-magnitude eigenvalues. For example, Figure 3-3 compares the singular spectrum of the sinc function to that of white Gaussian noise (WGN). WGN is maximally complex, so its spectrum contains the maximum number of 
eigenvalues with small, comparable magnitudes. However, the sinc function is simple, so its first eigenvalue's magnitude is nearly maximized while the few other eigenvalues' magnitudes are nearly zero.
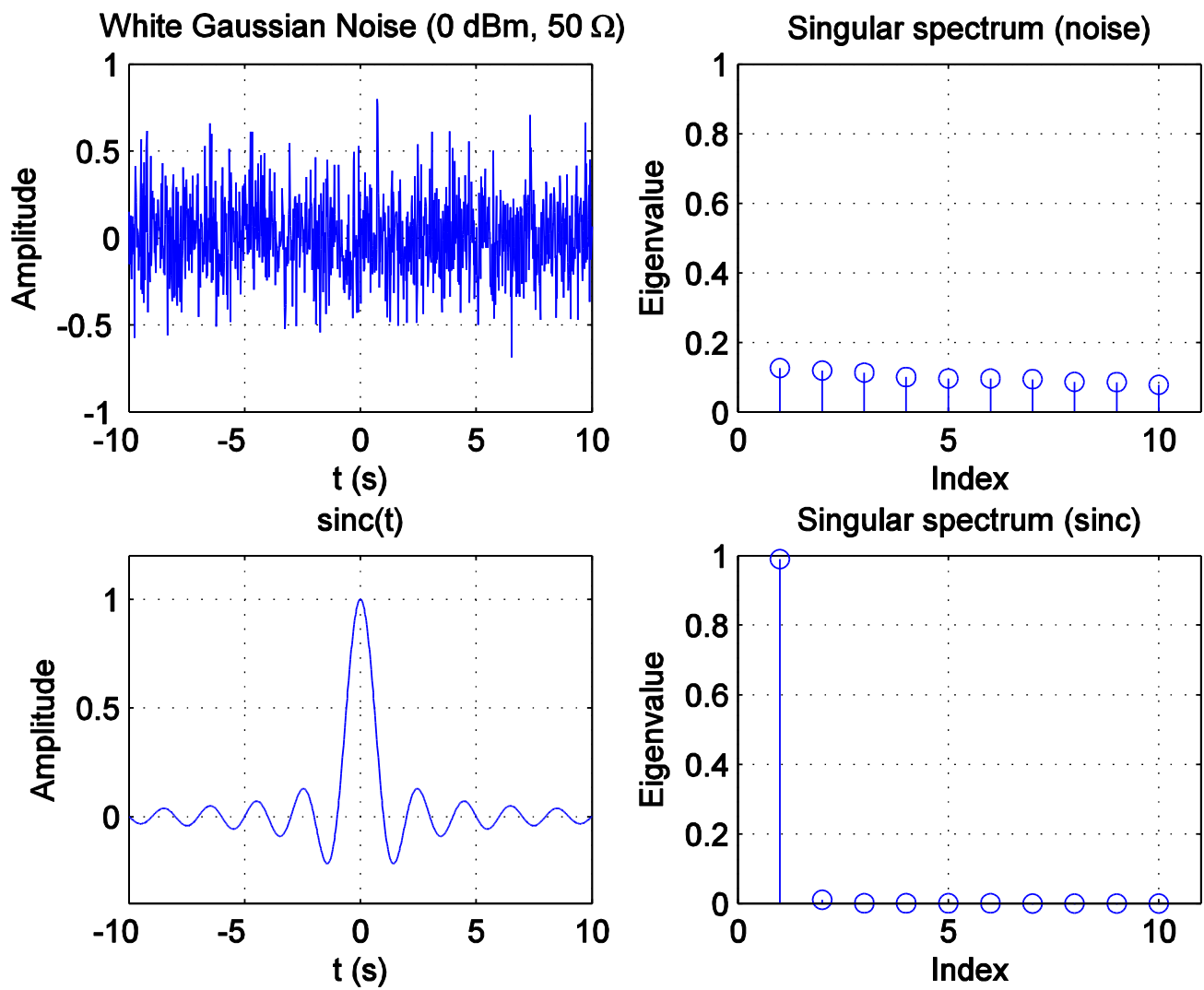

Figure 3-3: Singular spectra comparison [singular_spectra.m].

\subsubsection{Shannon Entropy}

The relationship between the singular spectrum and signal complexity mirrors the relationship between the probability mass function (pmf) of a random experiment and the uncertainty of the experiment's outcome. The information content $I$ is the number of symbols required to represent an outcome given a symbol set of size $b$ and a sample space with $K$ outcomes. For example, one bit is sufficient to represent the outcome of a coin flip but not the outcome of a six-sided die roll. The first bit distinguishes two equiprobable events, such as sides 1-3 and 4-6. A second bit distinguishes side 1 from 
sides 2-3 and side 4 from sides 5-6. If the roll's outcome is neither side 1 nor 4 , then a third bit distinguishes side 2 from 3 and side 5 from 6 . Thus, the information content is either two or three bits, depending upon the outcome of the roll.

For an experiment with a uniform probability distribution, the average information content is determined from the probability of a single outcome:

$$
\begin{gathered}
b^{I}=K=\frac{1}{P} \\
I=\log _{b} \frac{1}{P}=-\log _{b} P
\end{gathered}
$$

In general, the minimum entropy $H_{X}$ is the expected value of the information content of a random variable $X$ [30, pp. 202-203]:

$$
H_{X}=E\left[(I(X)]=\sum_{k=1}^{K} P[X=k] I(X=k)=-\sum_{k=1}^{K} P[X=k] \log _{b} P[X=k]\right.
$$

In other words, it represents the minimum average number of symbols required to represent an outcome of the random variable [30, p. 209]. For example, imagine picking a letter from a random word. The probability of a letter occurring is not the same for every letter, so assume the probability is $10 \%$ for each vowel, $2.5 \%$ for each consonant other than $X$ or $Z$, and $1.25 \%$ for $X$ and $1.25 \%$ for $Z$. Instead of using the first bit to divide the alphabet into two events of thirteen letters each, it is more informationally efficient to separate the letters into two equiprobable events. Thus, the first bit distinguishes vowels from consonants because the probability of picking a vowel is $50 \%$ and the probability of picking a consonant is $50 \%$. Likewise, the second bit divides the vowels and consonants. The probability of choosing the first ten consonants is $25 \%$, and the probability of choosing the last eleven consonants is also $25 \%$ (owing to the lower probability of $\mathrm{X}$ and $\mathrm{Z}$ ). However, the five vowels cannot be grouped equiprobably, so the average entropy is 
greater than the minimum average entropy. This process of subdividing the events continues until the decision tree ends in the outcomes (letters). The minimum entropy of the experiment with non-uniform letter probabilities is:

$$
\begin{gathered}
H=P_{V} I_{V}+P_{C} I_{C}+P_{X Z} I_{X Z} \\
=-5(0.1) \log _{2}(0.1)-19(0.025) \log _{2}(0.025)-2(0.0125) \log _{2}(0.0125)=4.35 \text { bits }
\end{gathered}
$$

In comparison, the minimum entropy of the same experiment with uniform letter probabilities is:

$$
H=-\log _{2}(26)=4.70 \text { bits }
$$

Thus, for a given random variable, a uniform pmf indicates maximum entropy while a nonuniform pmf indicates lower entropy. Likewise, for a given trajectory matrix, a uniform singular spectrum indicates maximum complexity while a non-uniform singular spectrum indicates lower complexity. In order to quantify complexity, the eigenvalues are normalized so that they sum to one and are then substituted for probabilities:

$$
P_{k}[M=i]=\hat{\lambda}_{i}(k)=\frac{\lambda_{i}(k)}{\sum_{i} \lambda_{i}(k)}
$$

where $k$ is the sample location of the window and $M$ is the eigenvalue index (the "random variable"). The eigenvalues are used to calculate the entropy of each window:

$$
\begin{aligned}
H_{M}(k) & =-\sum_{i} P_{k}[M=i] \log _{2} P_{k}[M=i] \\
& =-\sum_{i} \hat{\lambda}_{i}(k) \log _{2} \hat{\lambda}_{i}(k)
\end{aligned}
$$

and the entropy is used to determine the complexity $\Omega(k)$, which is the average number of hidden states in the dynamical system: 


$$
H_{M}(k)=\log _{2} \Omega(k) \rightarrow \Omega(k)=2^{H_{M}(k)}
$$

Finally, the simplicity is the inverse of complexity:

$$
\operatorname{simpl}(k)=\frac{1}{\Omega(k)}
$$

\subsection{Piecewise Constant Denoising}

The nonlinear simplicity transform is a fundamental algorithm for the comprehensive PCG segmentation system implemented and tested in this study. Even though the simplicity transform is more computationally demanding than conventional linear filters, such as those that use the Fast Fourier Transform (FFT), it can more effectively separate different sound signatures.

Ideally, each different sound segment in the simplicity-transformed PCG (S1, S2, etc.) would be represented by a unique, constant simplicity value or level. Instantaneous transitions between simplicity levels would then occur at the boundaries between different sound segments at easily identified jump locations. The transformed PCG would then resemble a piecewise constant (PWC) or jump sparse signal, composed of only a few unique constant levels $[15$, p. 7]. The stratification of the PCG simplicity into distinct levels in this way enables sound segmentation and classification of the more complex murmurs and sounds by simple thresholding. However, since the raw simplicity values do not provide an ideal piecewise constant representation (as seen in Figure 3-4), it is necessary to first filter the noise in the raw simplicity values before attempting sound segmentation on the waveform. 

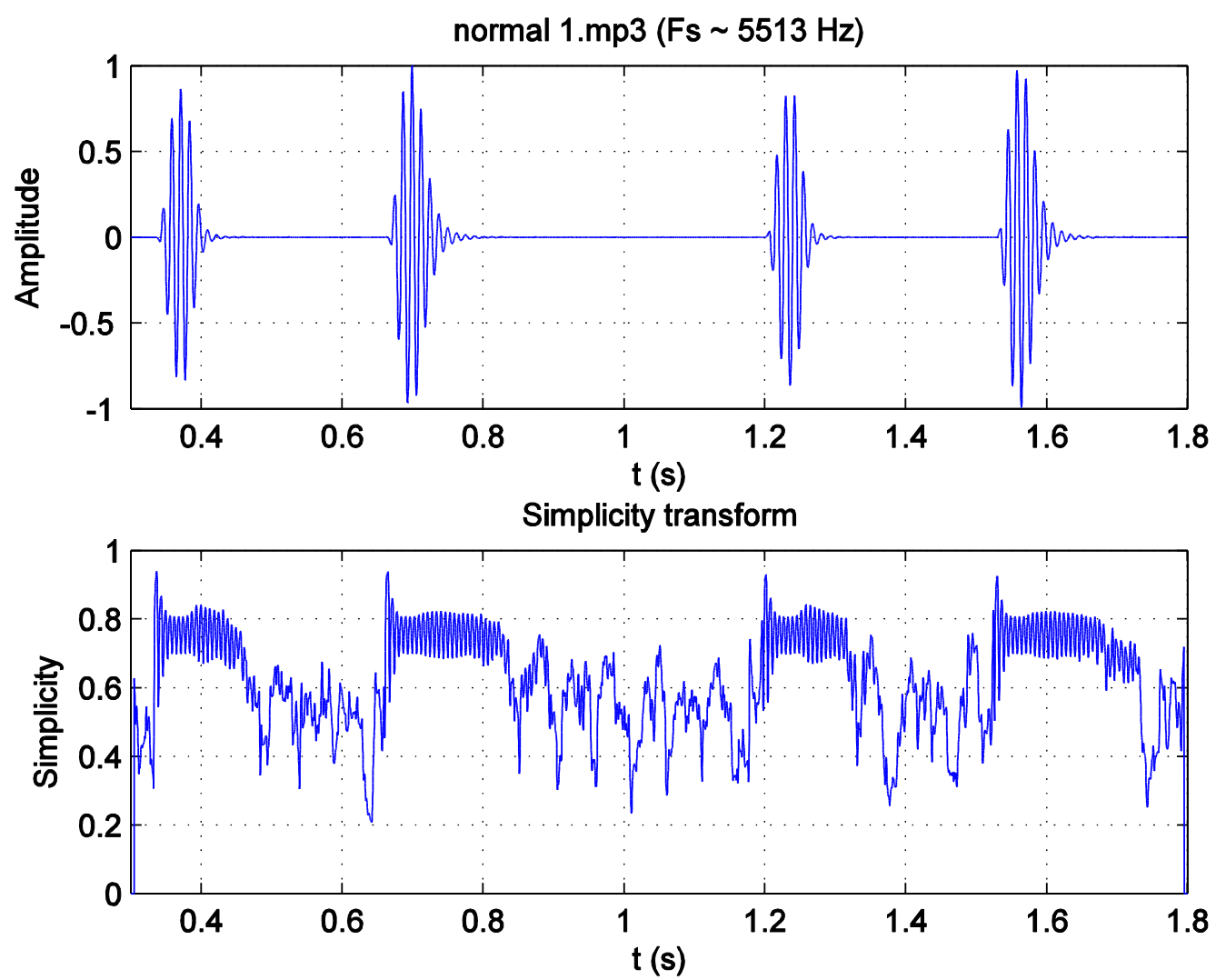

Figure 3-4: Raw simplicity waveform [PCG_simpl.m].

Smoothing filters, such as the moving average filter, reduce noise by locally averaging the time domain features in a waveform. Low pass filters (LPF), however, are superior to simple moving averages for most signals because they can more selectively attenuate the high frequency noise while preserving the low frequency features. Unfortunately, traditional low pass filtering is not suitable for a noisy piecewise constant signal with many jump discontinuities, as it will distort the sharp edges at the jump locations which contain high frequencies, and add ripple variations to the piecewise constant amplitude levels.

Since a PWC signal is a superposition of scaled and shifted rectangle functions, the effects of filtering can be illustrated on a single rectangular pulse. The Discrete Fourier Transform (DFT) of a rectangular pulse is a periodic train of discrete sinc functions that repeats at multiples of the sampling frequency. An ideal LPF truncates both the high 
frequency noise and the high frequency side lobes of the sinc function. After applying the inverse DFT to the filtered frequency domain representation, the output resembles a PWC signal, but with ripple throughout, ringing on the edges, and smoothed jumps. This is similar to the Gibbs phenomenon from the Fourier series, as shown in Figure 3-5. Thus, it is impossible to recover a noiseless PWC signal using linear frequency domain filtering, so other noise reduction techniques are required.

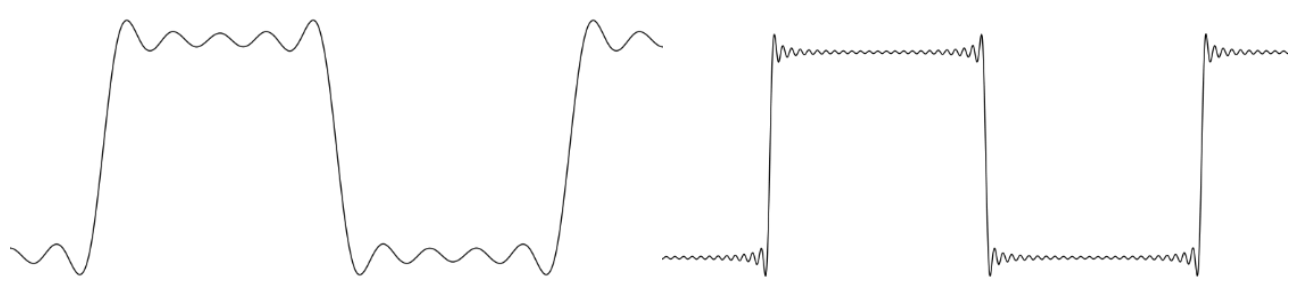

Figure 3-5: Low pass filtering a rectangle causes ripple. The signal at the right was filtered with a higher order LPF than the signal at the left [31].

Piecewise constant denoising or jump sparse reconstruction is the process of recovering a noisy signal's optimal PWC representation. It is performed using either levelset recovery or PWC smoothing algorithms, depending upon the expected number of unique levels in the recovered signal [15, p. 5]. Level-set recovery uses clustering algorithms such as K-means or mean shift to separate the sample values into a limited number of levels, so that the jump locations are found only after the levels are determined $[15$, p. 6]. This technique excels at detecting rapid fluctuations between levels, but it is not suitable for PCG segmentation because heart sound and murmur segments are associated with a multitude of simplicity levels. PWC smoothing, however, iteratively estimates a set of jump locations and levels until the output is an optimal PWC approximation of the original signal $[15$, p. 6$]$. Being constrained by both jump locations and sample values incurs an additional, but justified, computational cost for PWC smoothing because it enables detection of a greater number of unique levels with greater accuracy than level-set recovery. For example, the simplicity of an extra heart sound 
(S3/S4) is only slightly greater than the simplicity of a normal heart sound (S1/S2), and the simplicity of a murmur is significantly less than that of any heart sound. Since neither extra heart sounds nor murmurs are guaranteed to exist, the algorithm must determine the number of levels automatically. However, when the simplicity variance is small, as in the case of normal and extra heart sounds, samples that should be placed in separate levels might be combined into one level. Thus, level-set recovery is inadequate for the segmentation and classification of heart sounds and murmurs.

Solvers are algorithms that minimize functionals [15, p. 7]. A functional transforms a vector input, which is an element of a vector space, into a scalar output. For jump sparse reconstruction, the solver minimizes the functional by choosing the optimal set of jump locations and level values. A multitude of solvers exist for both PWC smoothing and levelset recovery, but all are special cases of a generalized functional. An illustrative example of functional minimization is determining the shortest path between two points in Euclidean space. The path can be represented by an infinite number of curves, but the shortest path is uniquely represented by a line. The vector field is the set of all possible paths, the vector input is a single path, and the scalar output is length of the shortest path between the two points. In general, a functional minimizer is not unique, but the most common PWC solvers produce a convex set of functional outputs, such that each iteration of the solver approaches the absolute minimum of the functional.

The simplicity transform of a PCG can be represented as a PWC signal corrupted with additive noise:

$$
\mathbf{x}=\mathbf{m}+\mathbf{e}
$$

where $x$ is the raw simplicity, $m$ is the PWC simplicity, and $e$ is the noise. The discrete functional equation for jump sparse reconstruction is given as: 


$$
H[\mathbf{m}]=\sum_{i=1}^{N} \sum_{j=1}^{N} \Lambda\left(x_{i}-m_{j}, m_{i}-m_{j}, x_{i}-x_{j}, i-j\right)
$$

where $x$ is the input signal and $m$ is the output signal for the current iteration of the solver, so that the first iteration's input $x^{0}$ is the original signal, the last iteration's output $m^{k}$ is the final PWC approximation, and all other iterations' inputs and outputs are intermediate PWC approximations. The solver iterates until $H[m]$ reaches an absolute minimum, which is the only minimum if the functional is convex. The inputs to $\Lambda$ are the differences:

$$
d=\left\{\begin{array}{c}
x_{i}-m_{j} \\
m_{i}-m_{j} \\
x_{i}-x_{j} \\
i-j
\end{array}\right.
$$

The first three are value differences because they only apply to sample values while the last is a sequence difference because it only applies to indices. The two types of functions in $\Lambda$ are losses and kernels, both of which are non-negative functions of absolute difference or distance. Loss functions are typically of the form:

$$
L_{p}(d)=|d|^{p}
$$

where $p \in \mathbb{R}$. For the specific case $p=0$, the loss reduces to:

$$
L_{0}(d)=|d|^{0}=\left\{\begin{array}{l}
1(d \neq 0) \\
0(d=0)
\end{array}\right.
$$

Kernels are non-negative functions of loss and are classified as either value kernels or sequence kernels. Value kernels operate on value distances while sequence kernels operate on sequence distances. Hard kernels restrict the loss to a maximum distance while soft kernels modify the loss over distance. The hard kernel:

$$
K_{W_{1}, W_{2}, p}(d)=I\left(W_{1} \leq L_{p}(d) \leq W_{2}\right)=\left\{\begin{array}{l}
1,(\mathrm{~T}) \\
0,(\mathrm{~F})
\end{array}\right.
$$


uses the indicator function $I(\cdot)$ to set a hard limit on the acceptable range of losses. Conversely, the soft kernel uses a non-zero, continuous function to modify the loss:

$$
K_{p}(d)=f\left(L_{p}(d)\right) \geq 0
$$

Typically, the soft kernel diminishes in value as the loss increases. The decaying exponential is a commonly used soft kernel because the loss is relatively unchanged over a short distance but rapidly approaches zero thereafter:

$$
K_{p}(d)=\exp \left(-\beta L_{p}(d)\right)
$$

Kernels are further classified as either local (at least one sample value is non-unity) or global (all sample values are unity).

All terms in $\Lambda$ are the product of a kernel and a loss. The loss directly contributes to the functional sum while the kernel modifies or restricts the loss:

$$
\Lambda=L_{p}(d) K_{p}(d)
$$

A functional that only contains the regularization term:

$$
\Lambda=\frac{\left|m_{i}-m_{j}\right|^{p}}{p}
$$

is minimized when all samples in the output $\mathbf{m}$ are identical because the global kernel does not modify or restrict the distance between sample values. Conversely, a functional that only contains the likelihood term:

$$
\Lambda=\frac{\left|x_{i}-m_{j}\right|^{p}}{p} I(i-j=0)=\frac{\left|x_{i}-m_{i}\right|^{p}}{p}
$$

is minimized when the final output $\mathbf{m}^{1}$ is identical to the original input $\mathbf{x}^{0}$ because the sequence kernel restricts the input and output samples to identical indices. Neither the 
regularization nor the likelihood term alone is sufficient for producing a PWC signal, but the combination of the two terms produces a PWC output:

$$
\Lambda=\frac{\left|x_{i}-m_{i}\right|^{p}}{p}+\gamma \frac{\left|m_{i}-m_{j}\right|^{p}}{p}
$$

The regularization parameter $\gamma$ balances the tradeoff between data-fidelity (likelihood) and sparsity (regularization). Sparsity is maximized when the sample values are the same and minimized when the sample values are unique. Conversely, data-fidelity is maximized when the output matches the input. The likelihood and regularization terms oppositely influence the shape of the output, so the solver has to balance both requirements to minimize the functional and produce an optimal PWC representation.

When $\gamma$ is zero, the solver does not attempt to minimize the distance between sample values. As $\gamma$ increases, the regularization term increases its contribution to the functional sum, which forces the solver to minimize the distance between output value samples. As $\gamma$ approaches infinity, all output sample values approach the same value. The constant value that minimizes the functional, then, is the average value of the original signal:

$$
\lim _{\gamma \rightarrow \infty} \Lambda=\frac{\left|x_{i}-c\right|^{p}}{p}
$$

A judicious choice of $\gamma$ will balance the influence of both the likelihood and regularization terms so that the optimal PWC signal has the appropriate balance of sparsity and datafidelity.

\subsection{Potts Functional}

The Potts functional is suitable for jump sparse reconstruction of the simplicity waveform because it models the input as a blurred PWC signal corrupted with additive noise [17, p. 3654]: 


$$
\mathbf{x}=\mathbf{a} * \mathbf{m}+\mathbf{e}
$$

Like before, $\mathbf{x}$ is the raw simplicity, $\mathbf{m}$ is the PWC signal, and $\mathbf{e}$ is the additive noise. The blurring is modeled as the convolution of the blur kernel a with the PWC signal. The blur kernel is not typically known a priori but is commonly assumed to be Gaussian:

$$
G(x)=\frac{1}{\sqrt{2 \pi \sigma^{2}}} e^{-\frac{x^{2}}{2 \sigma^{2}}}
$$

where $\sigma$ is the standard deviation. Alternatively, convolution can be reformulated with matrix multiplication:

$$
\mathbf{x}=\mathbf{A m}+\mathbf{e}
$$

Here the blur kernel a is converted to the blur matrix $\mathbf{A}$, which takes the form of a Toeplitz matrix:

$$
\mathbf{A}=\left[\begin{array}{cccccc}
a_{0} & a_{-1} & a_{-2} & \cdots & \cdots & a_{-(n-1)} \\
a_{1} & a_{0} & a_{-1} & \ddots & & \vdots \\
a_{2} & a_{1} & \ddots & \ddots & \ddots & \vdots \\
\vdots & \ddots & \ddots & \ddots & a_{-1} & a_{-2} \\
\vdots & & \ddots & a_{1} & a_{0} & a_{-1} \\
a_{n-1} & \cdots & \cdots & a_{2} & a_{1} & a_{0}
\end{array}\right]
$$

The sums in the original discrete functional equation can removed when the losses and kernels are expressed as vector operations. The loss function takes the form of a p-norm:

$$
\begin{gathered}
L_{p \neq 0}(\mathbf{d})=\|\mathbf{d}\|_{p}=\left(\sum_{i=1}^{n}\left|d_{i}\right|^{p}\right)^{\frac{1}{p}} \\
L_{0}(\mathbf{d})=\|\mathbf{d}\|_{0}=\sum_{i=1}^{n}\left|d_{i}\right|^{0}=\left|\left\{i: d_{i} \neq 0\right\}\right|
\end{gathered}
$$

The 0-norm counts the number of non-zero distances, which is represented by the cardinality operator (absolute value bars) applied to the set $\left\{i: d_{i} \neq 0\right\}$. 
Inverse Potts Minimization (iPotts) is the process of solving the Potts functional to recover an optimal PWC representation. The Potts functional is given as [17, p. 3654]:

$$
H[\mathbf{m}]=\gamma\|\nabla \mathbf{x}\|_{0}+\|\mathbf{A m}-\mathbf{x}\|_{p}^{p}
$$

The regularization term's 0-norm counts the total number of jumps:

$$
\begin{gathered}
\nabla x=x_{i+1}-x_{i} \\
\|\nabla \mathbf{x}\|_{0}=\sum_{i=1}^{N-1}\left|x_{i+1}-x_{i}\right|^{0}=\left|\left\{i: x_{i} \neq x_{i+1}\right\}\right|
\end{gathered}
$$

Instead of penalizing the height of each jump, the 0-norm penalizes all jumps equally so that the final PWC output is less likely to merge two levels separated by only a small jump height. The likelihood term reduces to:

$$
\|\mathbf{A m}-\mathbf{x}\|_{p}^{p}=\|\mathbf{b}-\mathbf{x}\|_{p}^{p}=\sum_{i=1}^{N}\left|b_{i}-x_{i}\right|^{p}
$$

Each iteration attempts to construct an improved PWC approximation $\mathbf{m}$ of the input $\mathbf{x}$ by minimizing the noise, which is the distance between aligned samples of the blurred PWC approximation and the input.

The MATLAB toolbox Pottslab [32] is used for jump sparse reconstruction in the segmentation system because it implements an efficient iPotts solver. Furthermore, Pottslab can solve univariate and multivariate signals, it allows the user to choose the likelihood term's p-norm, and it is robust enough to handle signals that have missing samples. This toolbox's primary benefit for PCG simplicity denoising is the choice of the p-norm, since the p-norm determines the noise model. 


\section{Segmentation System Implementation}

\subsection{System Overview}

\subsubsection{Introduction}

The algorithms and concepts introduced in Chapter 3 are combined in various ways to create a comprehensive heart sound and murmur segmentation system that is able to detect and classify normal heart sounds (S1/S2), split heart sounds (M1/T1, A2/P2), extra heart sounds (S3/S4, summation gallops), and murmurs (systolic/diastolic) from a raw PCG. The system is implemented in MATLAB (R2013b) for its seamless vector operations and data visualization, extensive signal processing toolboxes, and object oriented programming $(O O P)$ support.

The first operation is to load the PCG from the file system and remove any noise with a wavelet filter. Next, the heart sounds and murmurs are segmented using techniques specific to the particular segmentation function. The heart sound segments are then searched for split sound components and are consequently split into two segments are found. As a prerequisite to heart sound and murmur classification, the heart cycles are segmented by using the PCG's autocorrelation waveform to locate the cycle boundaries. The sound sounds in each heart cycle are then classified and stored in specific arrays such as S1, S2, systolic murmur, diastolic murmur, etc. Custom data types are provided for quickly determining the detected conditions and the heart cycles in which they are located. In addition, the segment arrays can be saved to the filesystem or plotted as color coded segments according to their sound type on the PCG.

\subsubsection{Properties and Methods}

Object oriented designs encapsulate, or combine, properties (data) and the methods (functions) that operate on those properties into a class. In MATLAB, each class has a 
single class definition file that is stored in the directory @classname. At a minimum, this file declares all properties and methods but may also set default property values and define methods. Any methods not fully defined in the class definition file must be implemented in separate files within the class directory. An object is an instance of a class and contains property values that define its state. The class constructor method initializes objects by assigning properties their default values or, for undefined properties, an empty double array. If additional functionality and arguments are required during object initialization, a custom constructor can be defined as:

$$
\text { obj = classname(args) }
$$

The software system for this project supports two distinct segmentation techniques that, while unique in implementation, retrieve the PCG waveform file in the same manner, depend on common parameters, store the results in the same format, and summarize and display the results identically. In addition, both techniques must be able to save the results to the file system and define default behaviors after reloading the results into the workspace. Thus, it is beneficial to encapsulate the system's functions and data in the class definition file stethoscope.m, where objects of this class are initialized as:

$$
\text { sscope }=\text { stethoscope(folder, file, varargin) }
$$

In a class definition file, properties are declared in blocks delimited by the properties end keywords, and functions are declared in blocks delimited by the methods end keywords. Attributes, contained in parentheses after the properties or methods keywords, modify how properties are accessed, altered, and stored, and how functions are accessed:

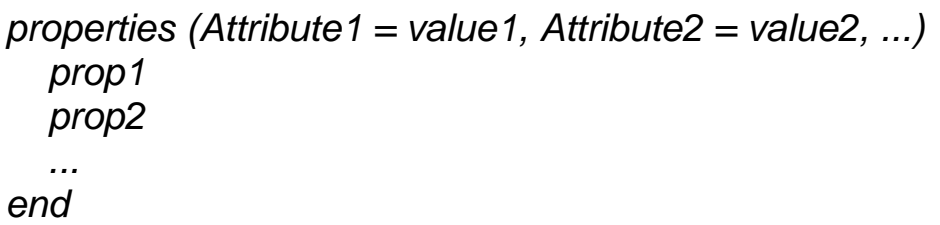




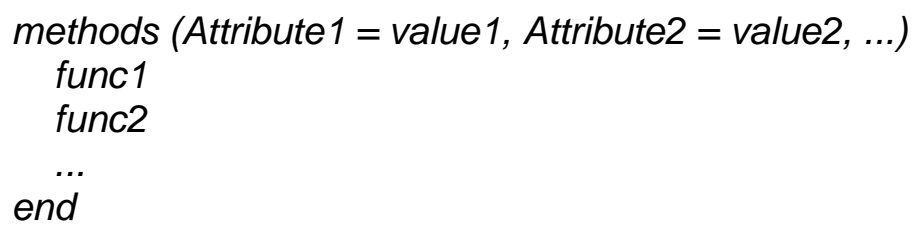

The attribute Access is common to both properties and methods. For methods, the default value public allows any function to call those methods, while the value private only allows class methods (methods within the class) to call those methods. For properties, Access is just a shortcut that sets the GetAccess and SetAccess attributes to the same value, where GetAccess controls which functions can query the property values, and SetAccess controls which functions can modify the property values i.e. change the object's state. The default value for both is public so that any function can read and write property values. This is not always desirable, as it is often necessary to restrict the ability to change state, so SetAccess=private only allows class methods to modify property values. Furthermore, GetAccess=private only allows class methods to query property values, but this attribute-value pair is not applied to any properties in this system because there is no requirement for hidden data. Finally, SetAccess=immutable only allows the class constructor to modify property values so that the values are permanent after object initialization.

By default, properties are stored in memory when an object is in the workspace and stored in a file when the object is saved. The attributes Transient and Dependent modify how properties are stored in both these cases. Transient properties are stored in memory but are not stored in the filesystem when the object is saved, and Dependent properties are never stored but instead calculated when accessed because they depend on the values of other properties. These attributes' values are logical, so they can either accept a Boolean value of true or false or are implicitly true when listed without a value. 
Methods are distinguished from functions by the manner in which they are called and their type of arguments. Since methods operate on objects, the first and only required argument to a method is an object of the method's class. However, static methods, or methods with the Static attribute, do not require this argument, as they are auxiliary methods that do not operate on an object of their class and are called as:

class_name.static_method(args)

The static method loadobj() loads object data from a file into an object in the workspace. Like the class constructor, loadobj() can be optionally defined for custom behavior, but unlike the constructor, loadobj() cannot be called directly.

The properties in stethoscope.m are classified as either constants or data. Constants are not literal constant data types, as their values can be changed, but are so named because the PCG retrieval, wavelet pre-filtering, and segmentation methods reference, rather than modify, their values. The constants file and folder are required arguments to the class constructor since they are empty by default, but even constants with non-empty default values can be changed through additional constructor arguments, namely, optional arguments and parameters. Optional arguments, if passed, are listed in a predefined order after the required arguments so that each value is assigned to the correct property. Parameters, however, may be listed in any order after the required and optional arguments since they are passed as name-value pairs in the format ('prop', val). The constants listed in Table 4-1 can only be set during initialization (SetAccess = immutable) whereas those listed in Table 4-2 can be set during and after initialization (SetAccess = public) using the dot notation sscope.prop=val. In both tables, the constants are grouped and labeled in accordance with the methods that reference them. 
In contrast to constant properties, data properties cannot be set directly because they contain the results generated from class methods (SetAccess $=$ private). These are listed in Table 4-3, where the properties that contain raw segmentation data are labeled as Segmentation Data, and the properties that contain data produced from other class methods or derived from the raw segmentation data are labeled in accordance with their attributes (either Dependent or Transient).

Table 4-1: stethoscope.m constant properties (SetAccess = immutable).

\begin{tabular}{|c|c|c|c|c|c|c|}
\hline & Properties & \multicolumn{2}{|c|}{ Type } & Argument & Default & Range \\
\hline \multirow{7}{*}{ 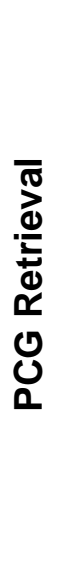 } & folder & char & vector & required & empty & NA \\
\hline & file & char & vector & required & empty & NA \\
\hline & path & char & vector & no & empty & NA \\
\hline & max_PCG_dur & double & scalar & parameter & 5 & positive reals \\
\hline & min_PCG_dur & double & scalar & parameter & 0 & {$\left[0, \max \_P C G \_d u r\right)$} \\
\hline & Fs_min & double & scalar & parameter & $4 \mathrm{kHz}$ & positive reals \\
\hline & ds_type & char & vector & parameter & 'dyadic' & $\begin{array}{l}\text { ‘dyadic' } \\
\text { ‘integer' } \\
\text { 'none' }\end{array}$ \\
\hline
\end{tabular}

Table 4-2: stethoscope. $\mathrm{m}$ constant properties (SetAccess $=$ public).

\begin{tabular}{|c|c|c|c|c|c|c|}
\hline & Properties & \multicolumn{2}{|c|}{ Type } & Argument & Default & Range \\
\hline \multirow{3}{*}{$\varliminf_{\frac{5}{0}}^{5}$} & show_filt & logical & scalar & parameter & false & true, false \\
\hline & wavef & char & vector & parameter & ‘db6’' & NA \\
\hline & $|v|$ & double & scalar & optional & 0 & nonnegative integers \\
\hline \multirow{5}{*}{ 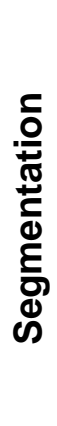 } & show_results & logical & scalar & parameter & false & true, false \\
\hline & max_HS_dur & double & scalar & parameter & $0.5 \mathrm{~s}$ & positive reals \\
\hline & min_HS_dur & double & scalar & parameter & $20 \mathrm{~ms}$ & positive reals \\
\hline & min_syst_dur & double & scalar & parameter & $100 \mathrm{~ms}$ & positive reals \\
\hline & min_murm_dur & double & scalar & parameter & $20 \mathrm{~ms}$ & positive reals \\
\hline
\end{tabular}


Table 4-3: stethoscope. $\mathrm{m}$ data properties $($ SetAccess $=$ private $)$.

\begin{tabular}{|c|c|c|c|c|}
\hline & Properties & \multicolumn{2}{|c|}{ Type } & Range \\
\hline \multirow{4}{*}{ 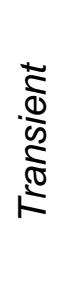 } & $P C G$ & double & vector & {$[-1,1]$} \\
\hline & filt_PCG & double & vector & {$[-1,1]$} \\
\hline & Fs & double & scalar & positive reals \\
\hline & $r$ & double & scalar & positive integers \\
\hline \multirow{4}{*}{$\begin{array}{l}\frac{\pi}{d} \\
\frac{0}{0} \\
\frac{1}{d} \\
\frac{0}{0} \\
0\end{array}$} & downsampled & logical & scalar & true, false \\
\hline & conditions & Map & vector & $\begin{array}{c}\text { 'Absent S1' } \\
\text { 'Absent S2' } \\
\text { 'Split S1' } \\
\text { 'Split S2' } \\
\text { 'S3' } \\
\text { 'S4' } \\
\text { 'Summation Gallop' } \\
\text { 'Systolic Murmur' } \\
\text { 'Diastolic Murmur' }\end{array}$ \\
\hline & short_list & char & vector & NA \\
\hline & num_cyc & double & vector & positive integers \\
\hline \multirow{8}{*}{ 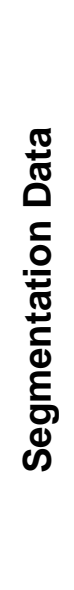 } & seg_method & char & vector & NA \\
\hline & cyc_bnds & double & vector & positive integers \\
\hline & $S 1, M 1, T 1$ & \multirow{6}{*}{ segment } & \multirow{6}{*}{ vector } & \multirow{6}{*}{ NA } \\
\hline & $S 2, A 2, P 2$ & & & \\
\hline & $S 3, S 4$ & & & \\
\hline & sum_gallop & & & \\
\hline & syst_murm & & & \\
\hline & diast_murm & & & \\
\hline
\end{tabular}

\subsubsection{PCG Retrieval}

The location of the PCG sound file is specified with path, which is automatically generated from the required constructor arguments folder and file. After setting any other constants passed as optional arguments, the constructor calls load_PCG(), which loads the PCG sound file but only stores the left, or first channel, in memory because the 
segmentation functions require a single channel. The min_PCG_dur and max_PCG_dur constants specify the lower and upper limits, respectively, of the PCG's duration in seconds, so the PCG is rejected if its duration is less than the minimum but is simply truncated if its duration exceeds the maximum. The PCG is also rejected if its sampling frequency is less than the minimum given by Fs_min. If $d s \_$type (down sampling type) is either 'dyadic' or 'integer', Ioad_PCG() calculates a down sampling factor $r$ such that the down sampled frequency is as close to, but not less than, Fs_min. The default value 'dyadic' restricts $r$ to positive powers of two for efficiency while 'integer' restricts $r$ to positive integers. Thus, if $r$ is greater than one, the sampling frequency is reduced by removing every $\mathrm{r}^{\text {th }}$ sample. If down sampling is unnecessary, it can be disabled by setting ds_type to 'none'.

The results of load_PCG() are stored in PCG, Fs, and $r$, all of which are Transient because it is inefficient to save the PCG samples when saving the object to the file system. Instead, the PCG can be reloaded exactly as before using load_PCG() since the constants that affect PCG retrieval are immutable and unchanged for the life of the object. Thus, load_PCG() is the first statement executed upon loading the object from the filesystem using the custom loadobj().

\subsubsection{Wavelet fittering}

The Discrete Wavelet Transform (DWT) is used to remove sharp edges and noise from the PCG before segmentation. The properties wavef and $I V I$ specify the wavelet function and decomposition level, respectively, to be used during wavelet filtering. The default wavelet function is ' $d b 6$ ' (Daubechies wavelet) because it closely resembles the morphology of S1 and S2, but the default level is zero so that the system does not attempt to filter the PCG unless the level is explicitly set. When the level is set to a positive integer,

the function $d w t$ filt() is called immediately after load_PCG() in the class constructor. The 
PCG is then "low pass" filtered by convolving the approximation coefficient at level $/ \mathrm{v} /$ with the low pass reconstruction filter and storing the result in filt_PCG. Thus, the high frequency features found in detail coefficients at current or lower levels are not included in the filtered PCG.

The process of choosing the approximation coefficient for reconstruction is demonstrated in Figure 4-1. $C D_{1}$ can be removed because it is almost pure noise and does not contain any discernible heart sound signatures. The heart sounds begin to appear in $C D_{2}$ because the lower end of the $C D_{2}$ frequency range is $689 \mathrm{~Hz}$. Nonetheless, $\mathrm{CD}_{2}$ can also be removed because its maximum amplitude is three order of magnitude less than that of $C A_{2}$, and the noise is still prevalent. The frequency band for $\mathrm{CD}_{3}$ is within the range of heart sounds and murmurs, the amplitude is only two orders of magnitude less than that of $\mathrm{CD}_{2}$, and the noise is small compared to the signal. Thus, the PCG is reconstructed from $C A_{2}$ alone, so $C D_{2}$ and $C D_{1}$ are removed, and frequencies greater than $689 \mathrm{~Hz}$ are excluded from the reconstructed the signal. The original and wavelet filtered PCG's are compared in Figure 4-2. This particular PCG contains little noise, but the filter at least smooths sharp edges, which is a necessary prerequisite for the segmentation functions. 

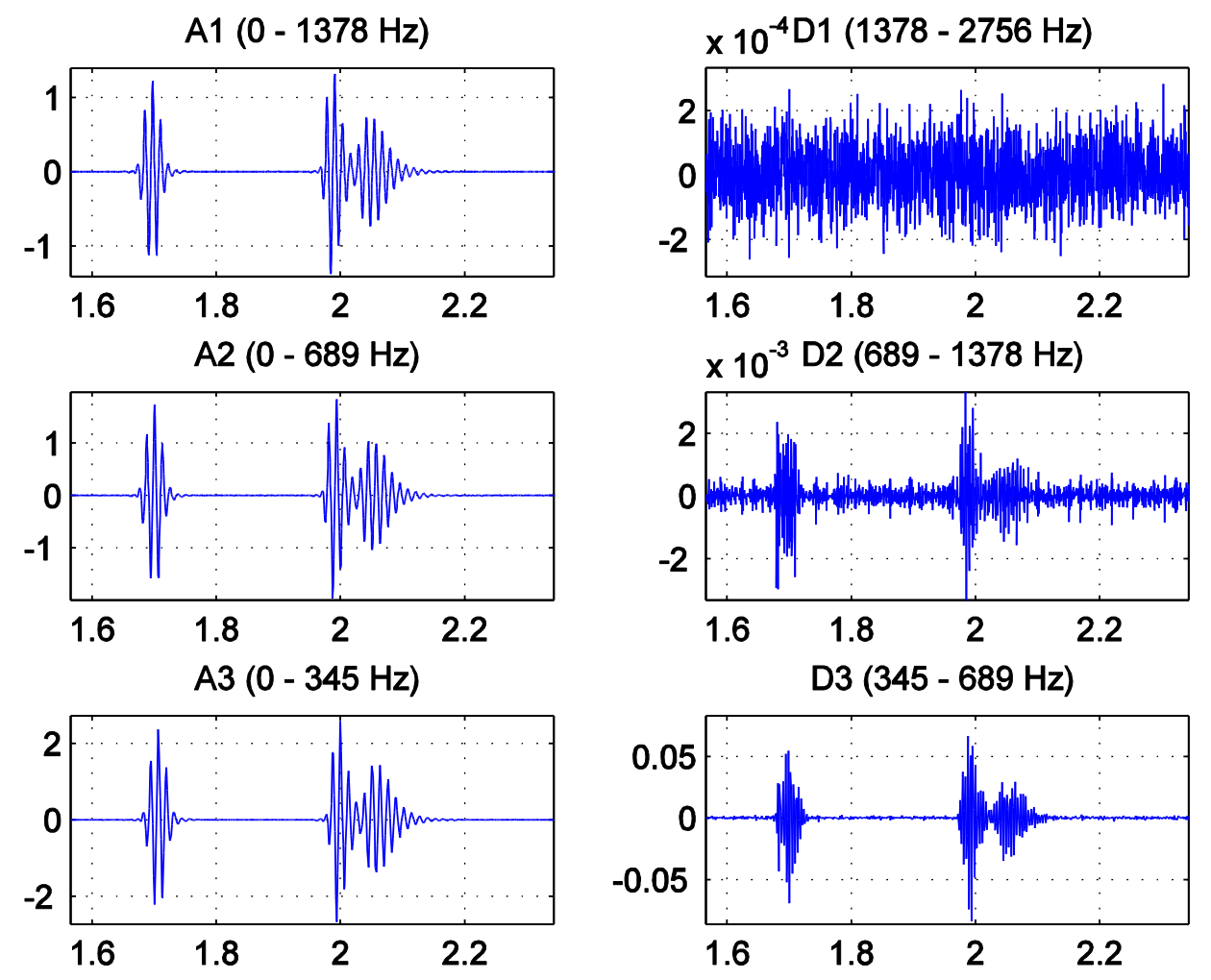

Figure 4-1: A range of approximation coefficients (left subplots) and detail coefficients (right subplots) are used to determine which approximation coefficient is optimal for PCG reconstruction [chp4_seg.m]. 

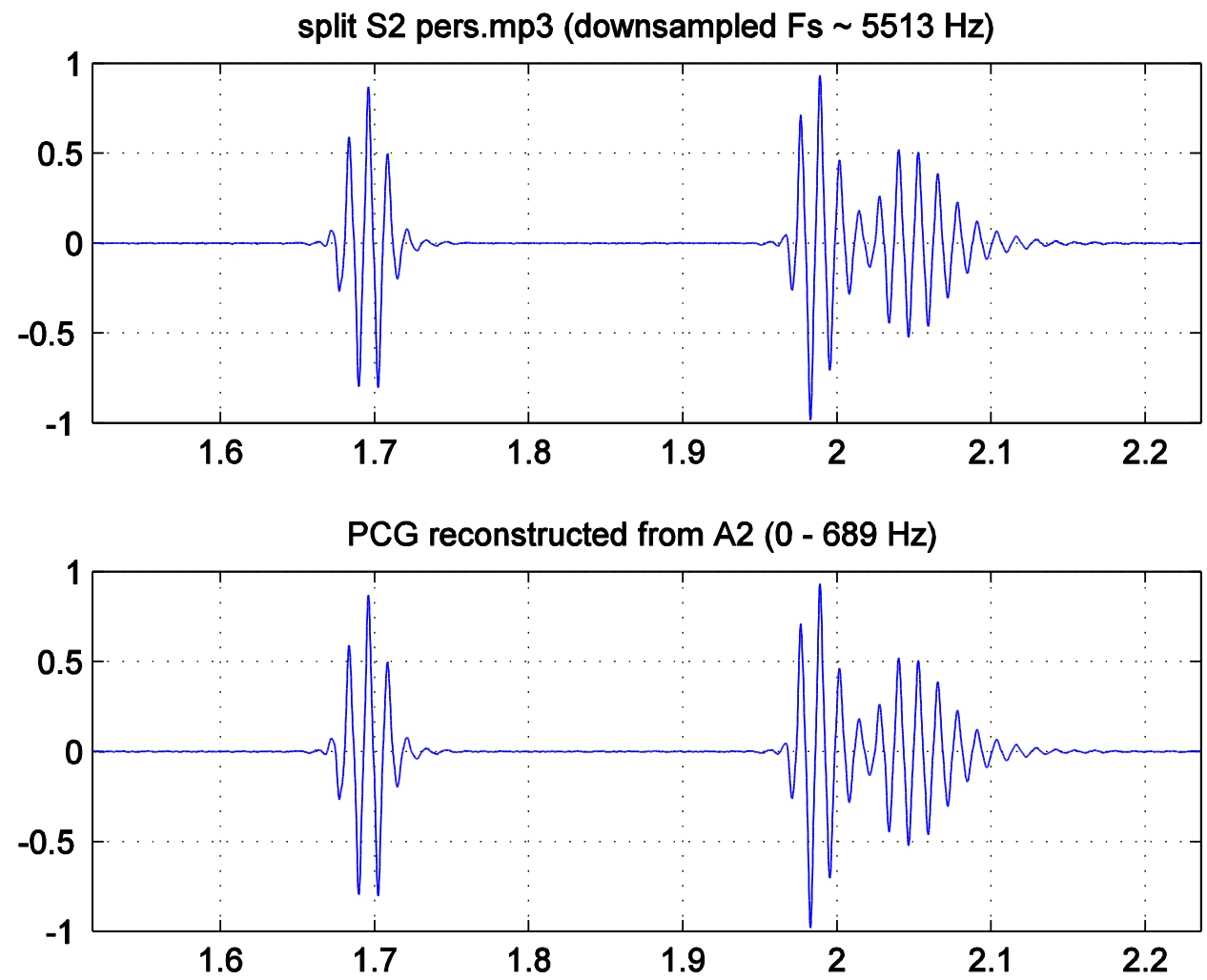

Figure 4-2: The original PCG (top subplot) has very little noise, so the filtered PCG (bottom subplot) appears similar to the original PCG [chp4_seg.m].

\subsubsection{Segmentation}

The segmentation functions require a special class, segment.m, for referencing and manipulating heart sound and murmur segments. In particular, this class stores the segment boundaries and provides methods for common segment operations. Objects of this class are initialized as:

$$
\text { seg = segment(strt, stop, mag) }
$$

where the required arguments strt and stop are the segment's integer start and stop indices, respectively; and the optional argument mag is a non-zero magnitude assigned to the segment, which has a default value of one. The constructor verifies that strt does not exceed stop and that both are positive integers since they reference array indices. In 
addition to storing segment boundaries, an object of this class can dynamically calculate the segment's duration in samples, seg.dur, and generate a vector of the segment's indices, seg.rng.

The first group of methods includes those that manipulate segments. The method:

$$
\text { seg = combine(seg, max_dur) }
$$

combines adjacent segments within max_dur samples of each other, where max_dur is an optional argument that has a default value of zero. Additionally, the method:

$$
\operatorname{seg}=\operatorname{split}(\operatorname{seg}, \mathrm{loc})
$$

splits segments apart at the sample locations given in the array loc such that the split segments are separated by one sample.

The second group of methods includes those that generate a signal from segments. The method:

$$
\operatorname{sig}=\operatorname{mask}(\operatorname{seg}, s z)
$$

creates a vector of dimension $s z$ that has a value of one for samples within the segment boundaries and a value of zero for samples outside of the segment boundaries. The method:

$$
\operatorname{sig}=\text { levels }(\operatorname{seg}, \mathrm{sz})
$$

does the same, except samples within the segment boundaries have a value of seg.mag. Finally, the method:

$$
\text { sig = signal(seg, ref, zero) }
$$

creates a copy of the reference signal ref, but sets samples outside of the segment boundaries to the value zero. This method is typically used to generate segment layers 
for a plot, where zero $=N a N$ so that samples between segments do not appear on the plot.

\subsubsection{Storing and summarizing results}

The segmentation results are stored in the sscope object in separate arrays named for the heart sound and murmur types. Six segment arrays are created for the normal heart sounds $(S 1, M 1, T 1, S 2, A 2, P 2)$ and three for the extra heart sounds $(S 3, S 4$, sum_gallop), where each array has the same number of elements as the number of detected heart cycles and contains at most one segment per heart cycle. Thus, the location of non-empty segments in an array indicate which heart cycles contain those segments, while the locations of empty segments in an array indicate which heart cycles do not contain those segments. The systolic and diastolic murmur segments are stored in two cell arrays (syst_murm, diast_murm) of the same length as the heart sound arrays, and each cell contains a variable length segment array since there can be more than one murmur segment in either systole or diastole.

Since it is difficult to determine which conditions are detected and in what heart cycle they are located from the raw heart sound and murmur arrays, stethoscope.m provides two Dependent properties, sscope.conditions and sscope.short_list, for consolidating and displaying these results, as well as the class method:

$$
\text { print(sscope) }
$$

The first dependent property, conditions, is a map object that extracts the most relevant information from the arrays. A map links each key in a keyset to a unique value. In MATLAB, the keys are strings, and the values are objects of a uniform type that are accessed using the keys as indices:

$$
\text { map_obj('key’) = value }
$$


For conditions, the keys represent the detected heart conditions (Table 4-4), and the values are numeric arrays that list the number of segments in each heart cycle.

Table 4-4: Set of all possible keys for conditions.

\begin{tabular}{|c|c|}
\hline Conditions & Keys \\
\hline Absent S1 & as1 \\
\hline Absent S2 & as2 \\
\hline Split S1 & ss1 \\
\hline Split S2 & ss2 \\
\hline S3 & s3 \\
\hline S4 & s4 \\
\hline Summation Gallop & $\mathrm{sg}$ \\
\hline Systolic Murmur & $\mathrm{sm}$ \\
\hline Diastolic Murmur & $\mathrm{dm}$ \\
\hline
\end{tabular}

The conditions keyset is empty when S1 and S2 are present in each heart cycle because this is the expected result for a healthy heart. As a result, 'as1' or 'as2' is only added to the keyset if an S1 or S2 segment is absent from at least one cycle. Also, 'ss1' denotes the presence of M1 and T1 segments, while 'ss2' denotes the presence of A2 and P2 segments. As an example, consider a PCG with four heart cycles and detected absent S2, split S1, and systolic murmur conditions. The keys and values are listed in Table 4-5.

Table 4-5: Example keys and values for sscope.conditions.

\begin{tabular}{|c|c|}
\hline Keys & Values \\
\hline 'as2' & {$\left[\begin{array}{llll}0 & 0 & 0 & 1\end{array}\right]$} \\
\hline 'ss1' & {$\left[\begin{array}{llll}1 & 1 & 1 & 1\end{array}\right]$} \\
\hline 'sm' & {$\left[\begin{array}{llll}1 & 2 & 2 & 2\end{array}\right]$} \\
\hline
\end{tabular}

Here, only the last cycle lacks an S2 segment, all cycles contain one M1 and one T1 segment, and the first cycle contains one systolic murmur while the other three cycles each contain two systolic murmur segments. Thus, conditions reduces nine segment arrays and two cell arrays, each of which typically contains many empty elements, into a single object that specifies the type, quantity, and heart cycle locations of all segments, which is adequate for diagnosing heart health. 
The function print(sscope) uses the data from conditions to list the number of: detected heart cycles (sscope.num_cyc), cycles with detected murmurs, heart cycles without detected murmurs, systolic segments with detected murmurs, and diastolic segments with detected murmurs. Using the example data from Table 4-5, the output of print(sscope) is:

\section{Cycles: 4}

With murmurs: 4

Without murmurs: 0

Syst murmurs: 4

Diast murmurs: 0

Syst+diast murmurs: 4

The other dependent property, short_list, is a string that summarize the segmentation results. It is a comma separated list of the keys, with the exception that when conditions is empty, the string is simply ' $h h$ ', which is short for "health heart". Using the same data from Table 4-5, the output of short_list is:

as2, ss1, sm

\subsubsection{Displaying results}

In addition to displaying textual results, the system can plot a PCG with segments color coded for the different heart conditions. The class method:

$$
\text { plot(sscope) }
$$

overloads the built in MATLAB function plot() to create a figure that contains two subplots: the first displays the PCG with red vertical lines marking the heart cycle boundaries obtained from cyc_bnds, and the second displays the segmented, color coded PCG without the cycle markers. The heart condition color codes are listed in Table 4-6. 
Table 4-6: Heart sound and murmur segment color codes for plot(sscope).

\begin{tabular}{|l|l|}
\hline Segments & Colors \\
\hline S1 & Blue \\
\hline S2 & Red \\
\hline S3 & Purple \\
\hline S4 & Green \\
\hline Summation Gallop & Black \\
\hline Systolic Murmur & Magenta \\
\hline Diastolic Murmur & Magenta \\
\hline
\end{tabular}

As an example, a figure with both systolic murmur and split $\mathrm{S} 2$ segments can be seen in Figure 4-3.
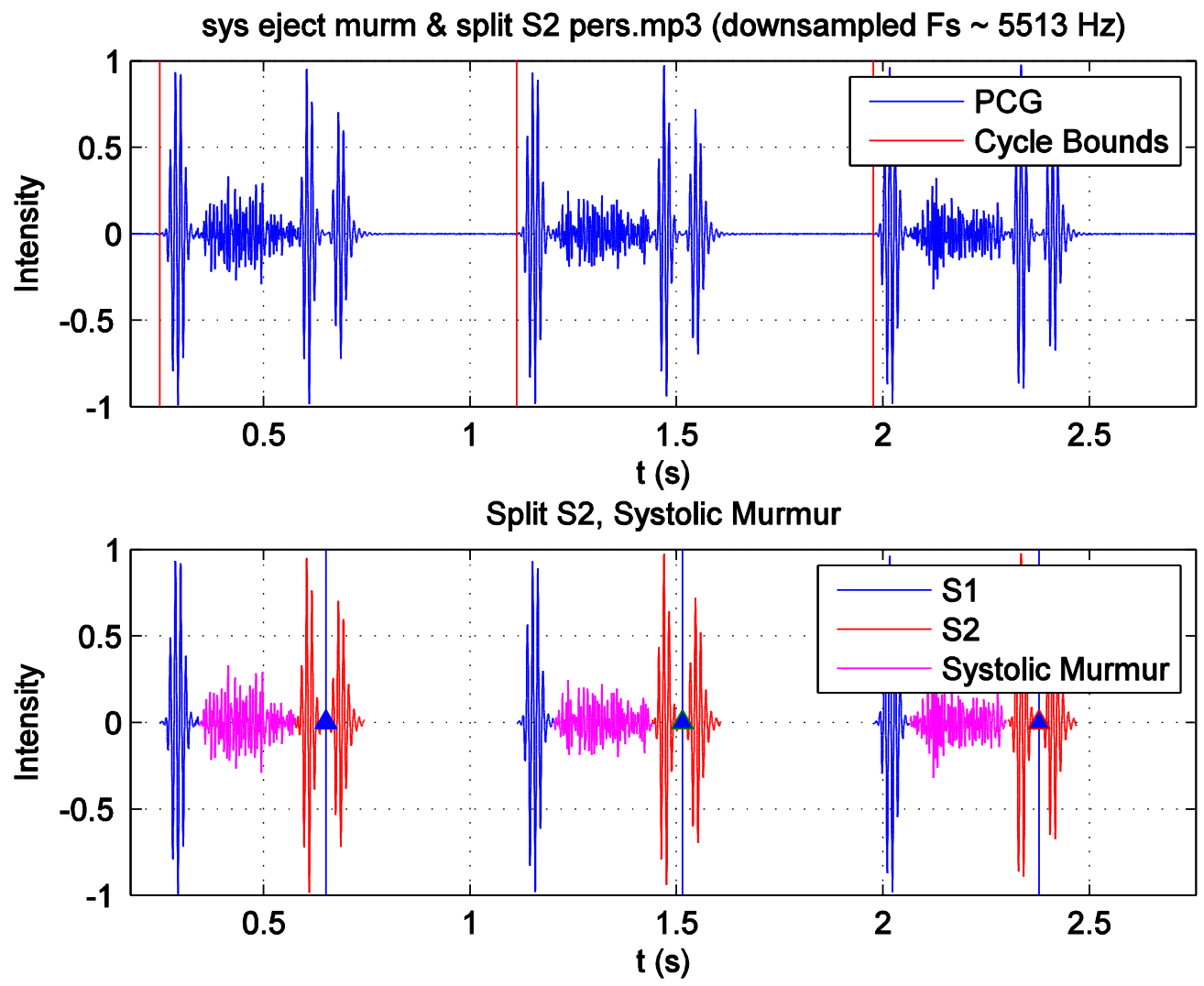

Figure 4-3: Graphical segmentation results for a PCG with systolic murmurs and split S2 [chp4_seg.m]. 


\subsection{Wavelet-based Segmentation}

\subsubsection{Heart Sound Segmentation}

The two segmentation functions developed for this project are differentiated by the specific algorithms used to distinguish heart sounds and murmurs, as this is the most fundamental operation for PCG segmentation. The function:

$$
\text { sscope }=d w t \_s e g m e n t(s s c o p e, I v l, \text { varargin) }
$$

attenuates the murmur samples using the discrete wavelet transform (DWT), so that the heart sounds can be segmented. As can be seen in Figure 3-1, the DWT is used in place of a frequency domain filter because the heart sounds and murmurs often overlap in frequency but have different time domain morphologies. This function's wavelet filter, like the stethoscope object's wavelet pre-filter, reconstructs a copy of sscope.PCG from its approximation coefficient at the decomposition level given by $I V I$. Since heart sounds and murmurs have lower frequencies than noise and sharp edges, and higher levels represent lower frequency bands, IVI must be greater than the pre-filter's level given by sscope.IVI.

The wavelet filter imperfectly attenuates the murmur samples, so any low amplitude samples must be zeroed through simple thresholding prior to heart sound segmentation. Since the PCG waveform oscillates between positive and negative values, the threshold is instead applied to the positive-valued energy waveform, which is typically calculated using the squared energy function. However, the squared energy function is unsuitable for thresholding here because it increases the energy separation between medium and high amplitude inputs significantly since its slope is directly proportional to amplitude. In

contrast, the Shannon energy $(S E)$ is minimized at small and large amplitudes but maximized at medium amplitudes:

$$
S E(x)=x^{2} \log x^{2}
$$


This is optimal for thresholding because the medium amplitude heart sounds are compressed into a smaller energy range, but not attenuated, while the low amplitude murmurs are assigned a low energy value. This creates an adequate separation between heart sounds and attenuated murmurs for simple thresholding. The Shannon energy and squared energy curves are compared in Figure 4-4 to demonstrate how the Shannon energy is maximized near the medium amplitudes while the squared energy continuously increases throughout the amplitude range.

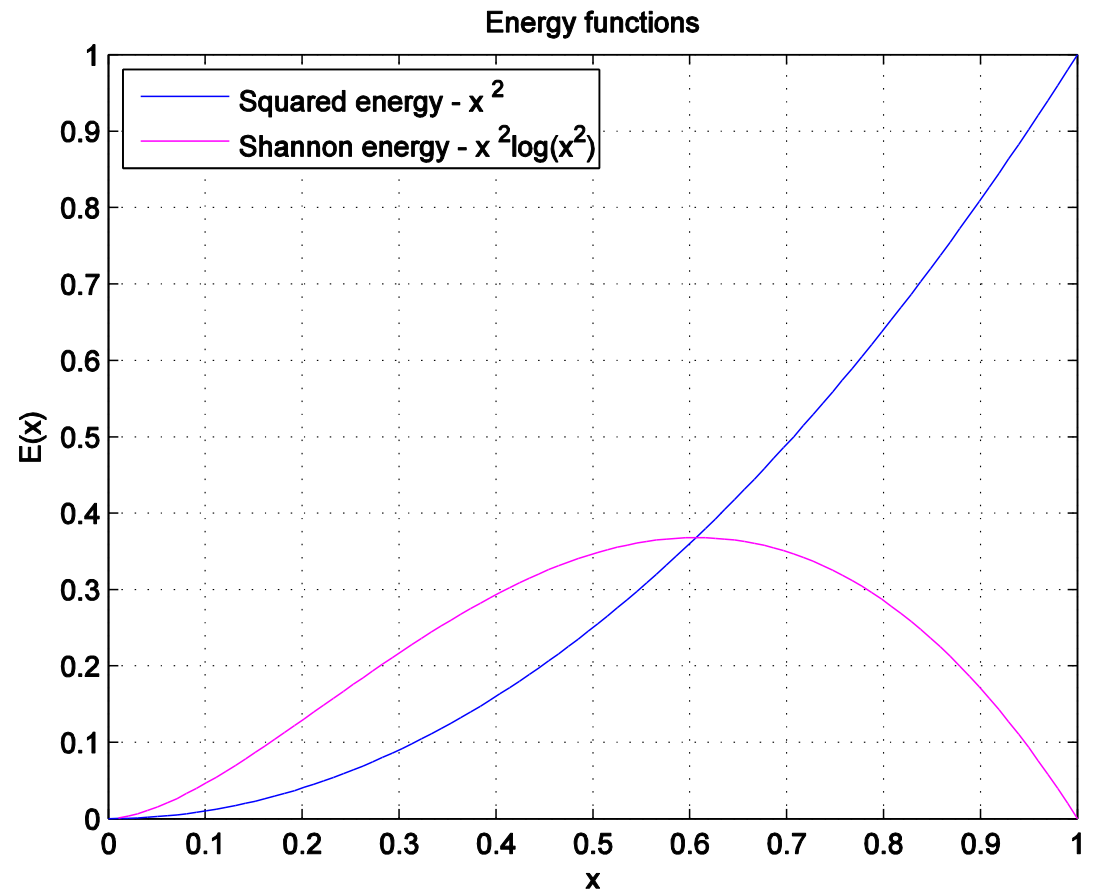

Figure 4-4: Shannon energy vs squared energy [energy_functions.m].

In order to prevent high amplitudes from being assigned low energy values less than the threshold, the Shannon energy waveform is obtained through a sliding window operation. The window averages the energy over a range of samples to reduce the effect of large-amplitude spikes in heart sound segments, and it "slides" or advances by one sample at a time to produce a large overlap that smooths the resulting energy waveform. 
Two separate thresholding operations are applied to the energy signal to zero the murmur samples. The first is the peak peeling algorithm adapted from [10], which zeroes the low energy samples between groups of contiguous higher energy samples, or peaks, through an iterative thresholding process demonstrated in Figure 4-5. The peak peeling function:

$$
\text { peaks = peak_peel }(x, S T C, \text { show, Fs })
$$

begins by applying a standard deviation threshold to the input signal $x$, and then stores the results in the peak signal and the rejected signal. The peak signal is a copy of the input except that sample values less than or equal to the threshold are zeroed (the blue portion of the waveform in subplot-1). After each iteration, the peak signal is added to a global output, in which the final output is a sum of all iterations' peaks (subplot-2). In contrast, the rejected signal is a copy of the input signal except that sample values greater than the threshold are zeroed (the red portion of the waveform in subplot-1). The rejected signal, as its name implies, is not added to the output like the peak signal but is instead the next iteration's input. The process only advances to the next iteration if the error, or absolute difference, between the mean square of the rejected signal and the mean square of the input signal is greater than the stopping condition given by STC (typically much less than one). Since the next iteration's input is always smaller than the current iteration's input, the error is reduced after each iteration and approaches the stopping condition. Thus, peak peeling adaptively determines an appropriate threshold for removing low energy samples. 

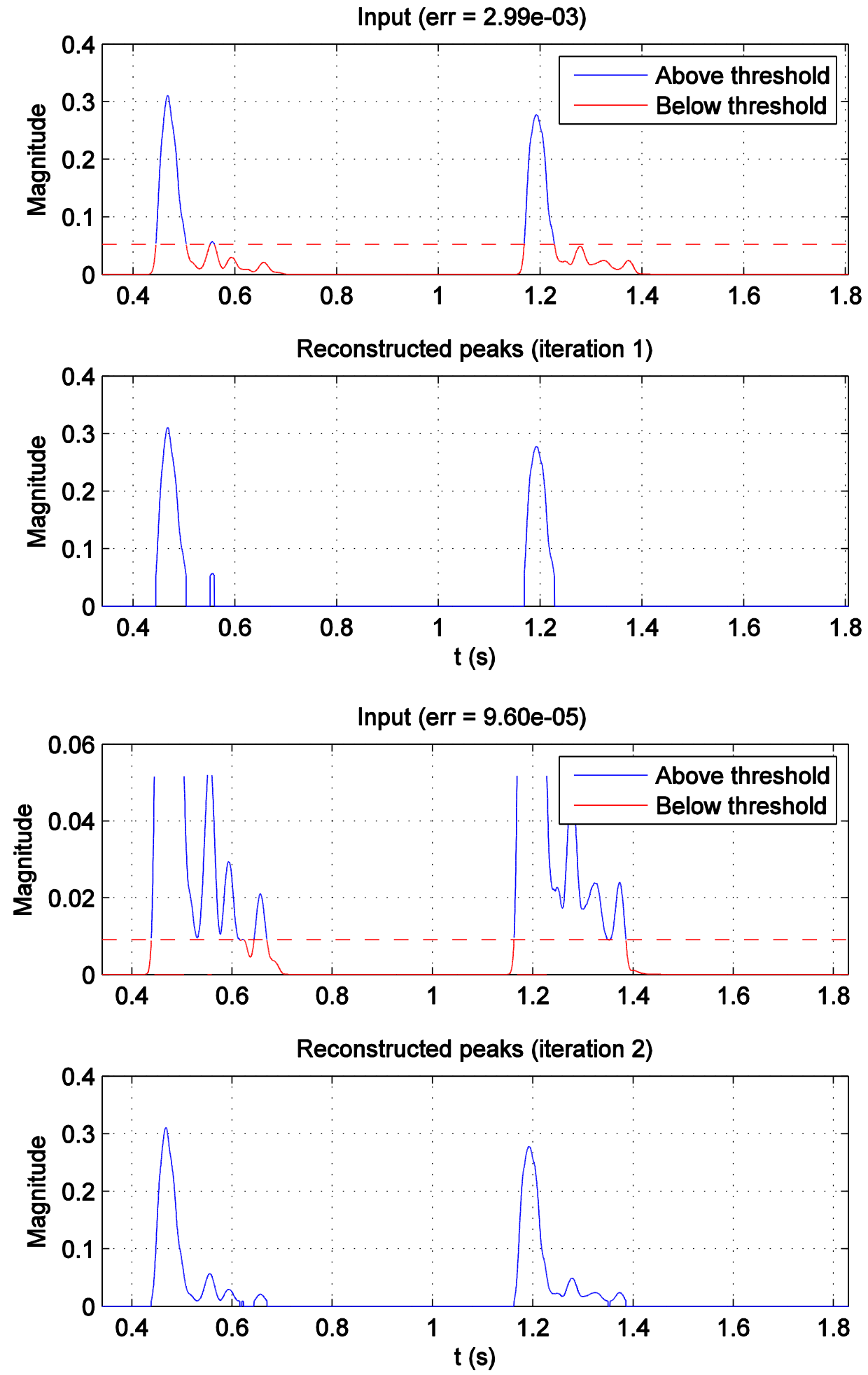

Figure 4-5: Two peak peeling iterations. Subplot-1 separates the input signal into the peak signal (blue) and the rejected signal (red). Subplot-2 displays the current output signal, which is a sum of the peaks from the current and previous iterations [chp4_seg.m]. 
The second threshold is a constant rather than an iterative algorithm and, unlike peak peeling, is applied to the maximum energy of each segment rather than the individual samples. Thus, the peaks acquired from peak peeling are segmented, and the segments with maximum energies less than the constant threshold are removed. The murmur attenuation operations are shown in Figure 4-6, which includes subplots for the PCG, wavelet reconstructed PCG, and peak peeled Shannon energy. In subplot-3, the segment that has a maximum energy less than the threshold is removed but nonetheless displayed on the plot to demonstrate the inadequacies of peak peeling and the necessity of the constant threshold. In addition to removing low energy segments, segments narrower than the minimum heart sound segment duration specified by sscope.min_HS_dur are also removed. The procedure for segmenting heart sounds is summarized in the list below:

1. Calculate the PCG's Shannon energy waveform

2. Peak peel the Shannon energy

3. Segment the Shannon energy peaks

4. Remove low energy segments

5. Remove short duration segments 

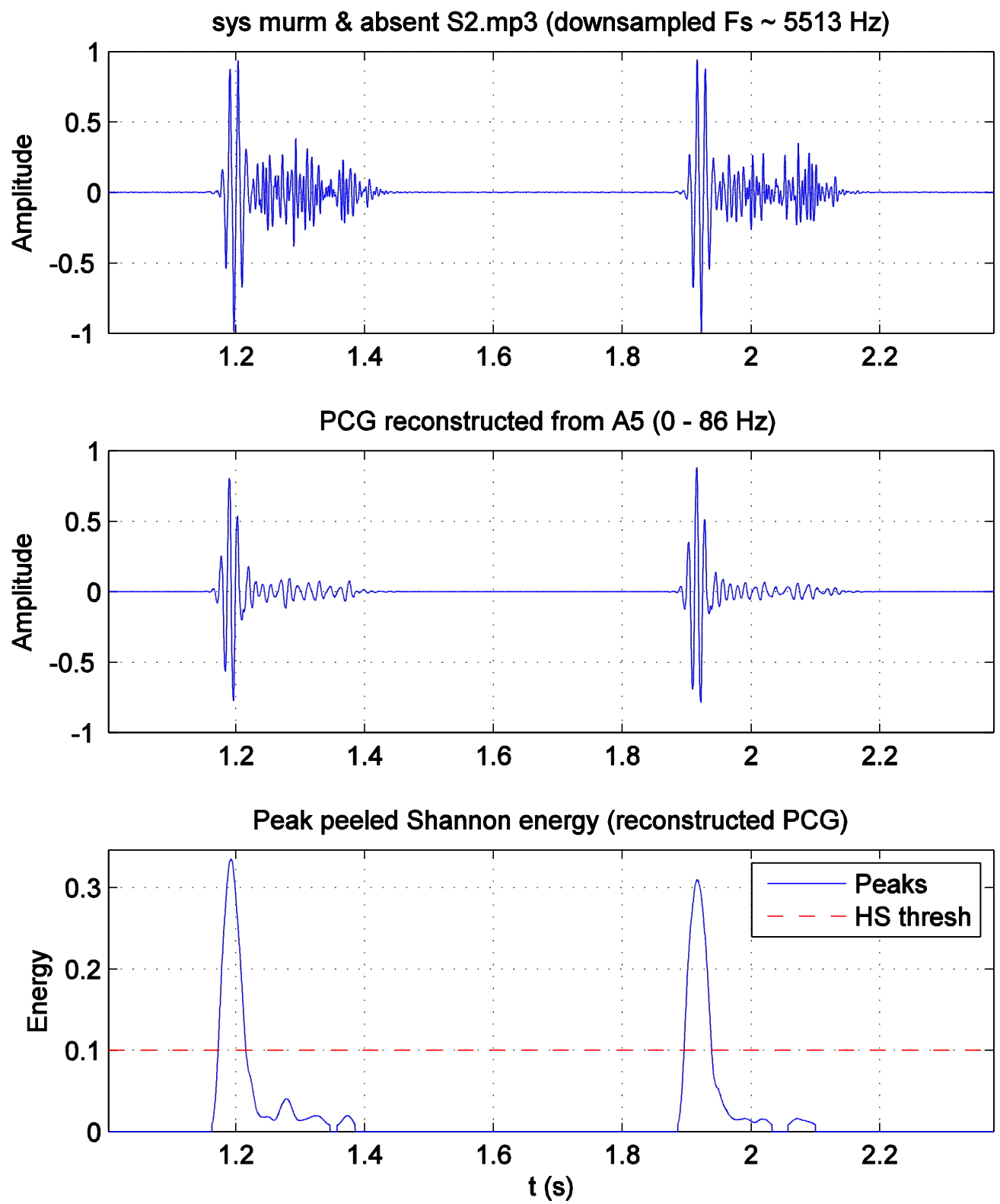

Figure 4-6: PCG (subplot-1), wavelet reconstructed PCG (subplot-2), and peak peeled Shannon energy with overlaid constant threshold (subplot-3) [chp4_seg.m].

\subsubsection{Removing Murmurs from Heart Sound Segments}

As can be seen in subplot-3 of Figure 4-6, incomplete murmur attenuation is problematic when murmur segments are connected to heart sound segments after peak peeling. However, there is typically a trough between the connected segments that can 
be used to reposition the segment boundaries and remove any murmur samples from the heart sound segments. The local function:

[new_HS,TR_LOC, thresh_lines] = trim_HS(HS, env, rel_thresh)

uses the PCG's envelope, env, to attempt to find the first trough to the left and to the right of the heart sound peak within each segment, and then it "trims" the HS segment boundaries at these trough locations. A heart sound peak is identified because its amplitude exceeds its containing segment's threshold given by rel_thresh, which is specified as a fraction of the segment's maximum amplitude. For segments containing multiple heart sound peaks, such as split heart sounds, only the envelope to the left of the leftmost peak and to the right of the rightmost peak is searched for troughs, so that troughs between heart sounds are not inadvertently marked. Within trim_HS(), the troughs are found using the function findpeaks() from the MATLAB signal processing toolbox. The modified segments are stored in new_HS, the trough locations are stored in TR_LOC, and information that can be used to plot the threshold lines is stored in thresh_lines.

The segment trimming process is illustrated in subplot-2 of Figure 4-7, where the heart sound segment boundaries obtained from peak peeling and constant thresholding are overlaid on the smoothed PCG envelope. The red vertical lines are placed on the segment boundaries, the horizontal magenta lines represent the peak threshold, and the yellow markers are placed on the detected trough locations. There are at most two troughs per segment, but each segment in Figure 4-7 only contains one because the attached murmurs are located to the right of the heart sounds. 

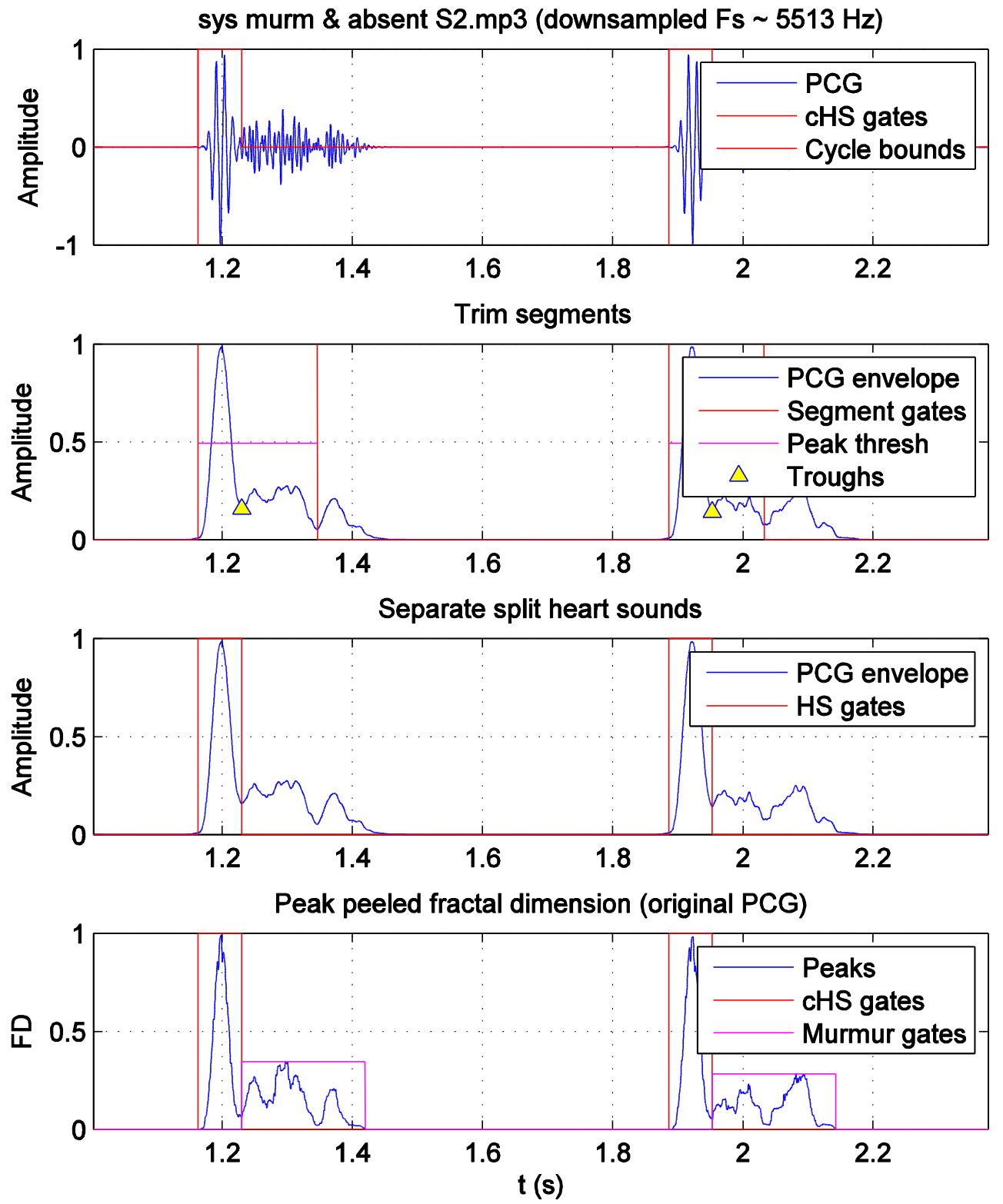

Figure 4-7: Final heart cycle and heart sound segment boundaries overlaid on the original PCG (subplot-1), troughs and thresholds for removing murmur samples from heart sound segments (subplot-2), murmur samples removed from heart sound segments (subplot-3), and segmented murmurs (subplot-4) [chp4_seg.m]. 


\subsubsection{Separating Split Heart Sounds}

After the heart sound segment boundaries are repositioned so as not to include any murmur samples, the heart sound segments containing split sounds must be divided into their split sound components. Split heart sounds are identified when the PCG envelope has two peaks of a sufficient height within a single heart sound segment. The function:

$\left[H S, T R \_L O C, P K \_L O C\right]=s p l i t \_H S(H S, e n v$, min_dist, min_height)

uses the PCG envelope, env, to find the two largest peaks in each heart sound segment that are separated by at least min_dist samples (typically set to sscope.min_HS_dur) and that exceed the magnitude min_height. After the peaks are located, the deepest trough between the two peaks is located, which is where the heart sound segment is split.

In subplot-2 of Figure 4-8, the split sound components are not marked by trim_HS() because they lie between two heart sound peaks. For split_HS() In subplot-3, the horizontal magenta line represents the trough threshold, red markers are placed on the peaks, yellow markers are placed on the troughs between the peaks, and red vertical lines denote the new segment boundaries. 

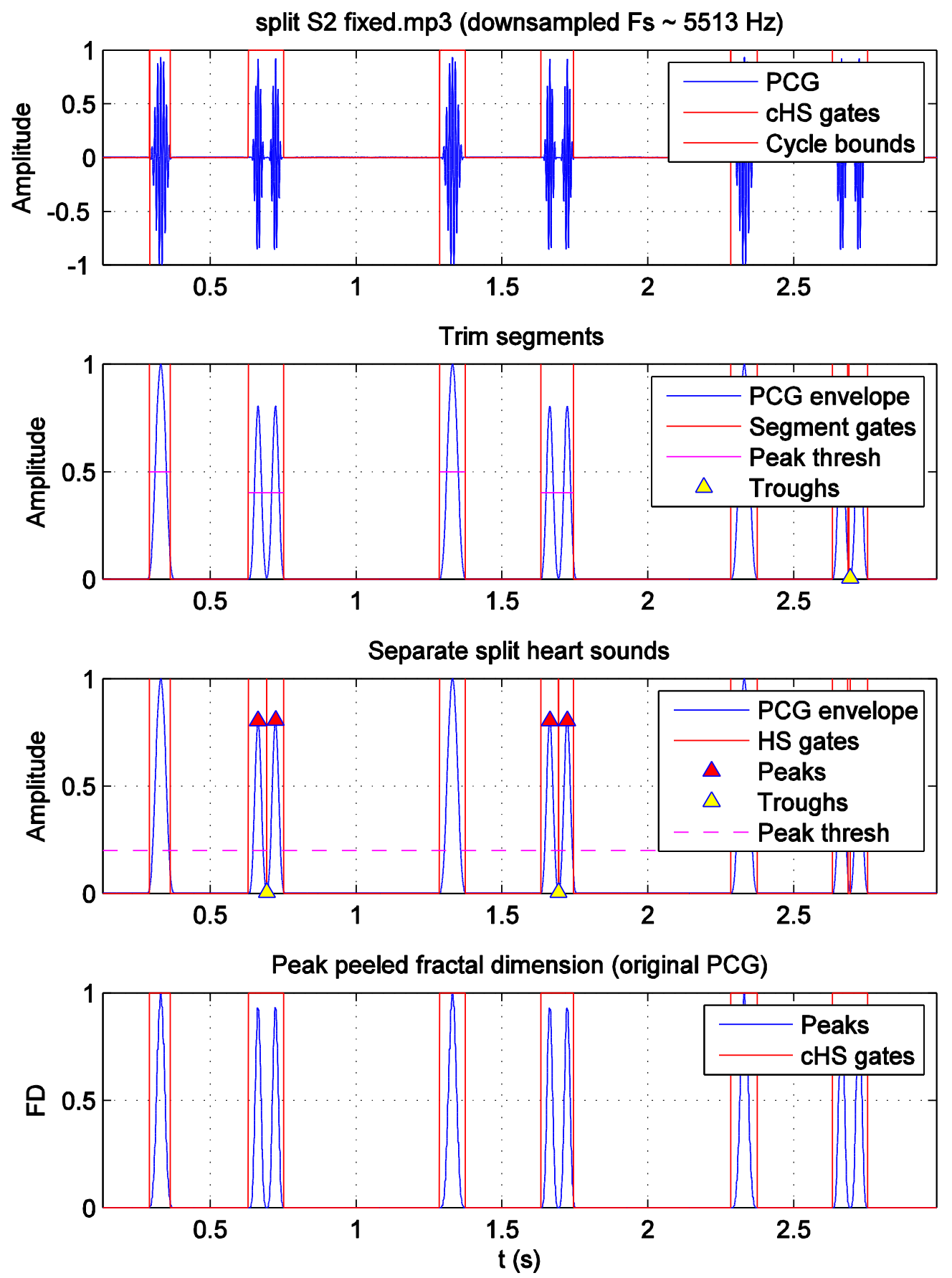

Figure 4-8: A heart sound segment containing split heart sounds is separated into its component segments (subplot-3) [chp4_seg.m]. 


\subsubsection{Heart Cycle Segmentation}

All heart sound segments obtained so far are stored in a single segment array but must eventually be classified and stored in separate arrays corresponding to a specific type (S1, S2, etc.). However, it is only possible to accurately classify and sort the heart sound segments after the heart cycles boundaries are known. The function:

$$
\text { cyc_bnds }=\text { find_heart_cycles(HS, PCG, min_dist, show, Fs) }
$$

uses periodic spikes in the autocorrelation of the PCG's envelope to locate the heart cycle boundaries. The autocorrelation compares two copies of the PCG at different time offsets, or lags, to discover periodicity in the signal; and it is applied to the PCG's envelope so as to prevent lulls in the output for lags where the PCG's oscillations are out of phase. As an error check, the spikes must be spaced apart by at least the minimum heart cycle duration min_dist, which is typically twice the minimum systole duration given by sscope.min_syst_dur.

In the autocorrelation waveform, the beginning of the first cycle is located at zero lag while the end of the first cycle is located at the first spike, which is the largest peak. Since the remaining spikes decrease in magnitude for increasing lags, and the two waveforms have little overlap for large lags, the last cycle boundary typically does not have a characteristic spike. Therefore, only the first cycle's boundary is placed on a spike, while the remaining boundaries are placed at integer multiples of the first spike's location. This process is illustrated in subplot-1 of Figure 4-9, where red markers lie on the detected peaks, and red vertical lines intersect the cycle boundaries.

Even though the spacing between heart cycle boundaries is consistent, the boundaries must be repositioned so that they align with the heart sound segments' start indices, as can be seen in subplot-2 of Figure 4-9. The cycle boundaries are shifted to the 
right by a duration equal to the offset of the first heart sound segment because the autocorrelation waveform only provides information about the relative, rather than the absolute, cycle boundaries. This shift places the first boundary on the first heart sound segment's start index, but the other boundaries do not necessarily lie on heart sound segment start indices. As a result, these boundaries are moved to the closest segment start index, and any boundaries shifted beyond the limits of the PCG are removed. To prevent cycle boundaries from being placed between split heart sound segments, segments spaced within min_dist samples of each other should be combined prior to calling find_heart_cycles(). 

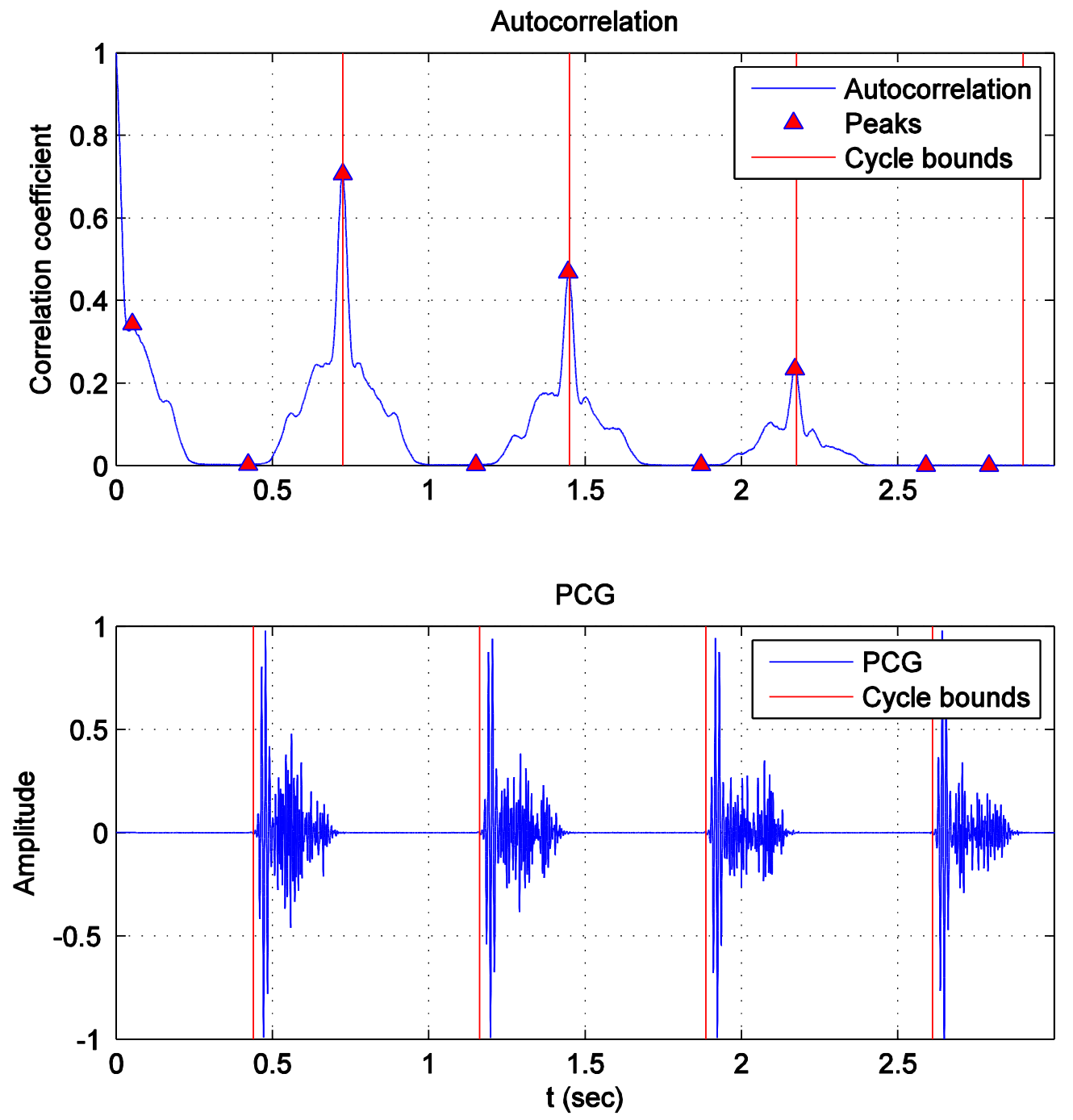

Figure 4-9: The heart cycle boundary locations are approximated from spikes in the autocorrelation of the PCG's envelope (subplot-1). Afterwards, the cycle boundaries are shifted right and aligned with the nearest heart sound segment start indices (subplot-2) [chp4_seg.m].

\subsubsection{Murmur Segmentation}

Since the heart sounds serve as the boundaries between which murmurs are detected, murmurs must be segmented after heart sounds. Murmur segmentation is similar to heart sound segmentation because a positive-valued waveform is peak peeled to detect the segment boundaries. However, the fractal dimension of the original PCG, rather than the energy waveform of the filtered PCG, is peak peeled because the fractal 
dimension attenuates noise to a greater extent. Thus, subplot-3 in Figure 4-6 is analogous to subplot-4 in Figure 4-7, as both contain a peak peeled waveform that is used to segment certain sounds. As with heart sounds, peak peeling is necessary to zero the low intensity samples in order to extract the sound segment boundaries, but the constant threshold is unnecessary here because murmurs do not have a minimum required amplitude or energy. After peeling, the murmur peaks are segmented, and any segments with durations less than sscope.min_murm_dur are removed.

\subsubsection{Heart Sound and Murmur Classification}

Only after segmenting the heart sounds, murmurs, and heart cycle boundaries is it possible to classify and store the segments in separate arrays corresponding to specific sound types. However, sound segment classification requires that no more than two normal heart sound segments representing S1 and S2 are present in each heart cycle. This total does not include split sound segments, as adjacent segments separated by less

than the minimum systole duration (sscope.min_syst_dur) are considered split sound components and grouped together for the purpose of identifying S1 and S2 segments. Thus, no more than two normal heart sound segments, or two groups of combined split sound segments, separated by at least the minimum systole duration may exist in a single cycle. The function:

$\left[H S, c H S, c y c \_b n d s\right]=$ limit_HS $\left(H S, c H S, c y c \_b n d s, E\right)$

discards any normal heart sound segments other than the two in each cycle that have the greatest maximum energy by referencing the energy waveform $\mathrm{E}$. The $H S$ segment array may contain split sounds since those are combined in the $\mathrm{cHS}$ array. Also, if the start index of a discarded segment lied on a heart cycle boundary, this function will shift the cycle boundary to the next segment's start index. Limiting each heart cycle to no more than two normal heart sound segments is essential for identifying systole and diastole, which are 
used to classify systolic and diastolic murmurs as well as the extra heart sounds S3, S4, and summation gallops.

After removing the excess heart sound segments, the function:

$[S 1, M 1, T 1, \ldots$

$S 2, A 2, P 2, \ldots$

S3, S4, sum_gallop, ...

syst_murm, diast_murm] $=$ Ibl_sounds $\left(H S, c H S, c y c \_b n d s\right.$, extra_HS, murm)

classifies the normal heart sounds, extra heart sounds, and murmurs; and transfers segments from the input arrays $H S$, extra_HS, and murm to the output arrays that correspond to specific sounds. Classification operates on a single heart cycle at a time because the process is dependent on the total number of normal heart sound segments in each heart cycle. When a cycle contains two normal heart sound segments, the duration between the stop index of the first segment and start index of the second segment is compared to the duration between the stop index of the second segment and the end of the heart cycle. Since these two durations represent either systole or diastole, the shorter duration is systole and the longer duration is diastole. If both durations are the same, the first is assumed to be systole and the second is assumed to be diastole. The normal heart sound segments can therefore be identified because $\mathrm{S} 1$ precedes systole and $\mathrm{S} 2$ precedes diastole. When a cycle only contains one normal heart sound segment, the segment is assumed to be S1, and the duration between the S1 stop index and the end of the heart cycle is assumed to be diastole.

After identifying the S1 and S2 segments, the local function:

$$
[C 1, C 2]=|b| \_s p l i t(H S, S)
$$

searches for split sound segments within the boundaries of a heart sound segment $(S)$. If two segments are found, then the first is stored in $C 1$ and the second is stored in $C 2$; if $S$ 
does not contain two segments, then $C 1$ and $C 2$ are uninitialized segments. This function searches $S 1$ for $M 1$ and $T 1$ and $S 2$ for $A 2$ and $P 2$ :

$[M 1, T 1]=l b l \_s p l i t(H S, S 1)$

$[A 2, P 2]=l b l s p l i t(H S, S 2)$

The systole and diastole segments are then used to classify the murmur segments. The local function:

$$
\text { murm_type }=\text { Ibl_murm(murm, sil) }
$$

searches for murmur segments within the boundaries of a silent segment (sil), and recovered murmur segments are stored in the variable length segment array murm_type. This function searches for both systolic and diastolic murmurs:

$$
\begin{aligned}
& \text { syst_murm }=|b| \text { murm (murm, syst }) \\
& \text { diast_murm }=|b| \_m u r m(\text { murm, diast })
\end{aligned}
$$

Finally, the extra heart sound segments are classified by their locations in diastole. The local function:

[S3, S4, sum_gallop] = Ibl_extra(extra, diast)

searches for extra heart sound segments within the boundaries of a diastole segment (diast). The murmur segments are classified by their locations relative to the center of diastole; in particular, S3 segments are located to the left of center, and S4 segments are located to the right of center. Summation gallops, being the superposition of S3 and S4, contain the center of diastole within their segment boundaries. In order to prevent erroneous detection, the number of segments in diastole limits sound classification behavior. When one segment is present in diastole, it may be classified as either an S3, an S4, or a summation gallop. When two segments are present, the first segment is S3, and the second segment is S4. If neither of these configurations are detected, an empty array is returned rather than an error. 
Unfortunately, dwt_segment() does not possess the ability to distinguish normal heart sounds from extra heart sounds, so the extra segment array passed to Ibl_sounds() will be empty. Nonetheless, Iblextra() is described here because it is a general technique that is also used in simplicity-based segmentation, as its inputs are segment arrays and cycle boundaries instead of implementation-specific waveforms

\subsection{Simplicity-based Segmentation}

The function:

$$
\text { sscope }=\text { simpl_segment(sscope, varargin) }
$$

utilizes the simplicity transform to segment heart sounds and murmurs as an alternative to wavelet-based segmentation. Many of the methods and concepts from wavelet-based segmentation are reused, but new techniques are developed as well. However, the primary factor that distinguishes simplicity from wavelet-based segmentation is that the heart sounds and murmurs are segmented in a single operation, which reduces overall complexity and improves the accuracy of the segment boundaries.

\subsubsection{Simplicity Waveform Filtering}

An ideal piecewise constant (PWC) function is composed of a series of constant levels separated by instantaneous transitions at the jump locations. For the simplicity waveform, the segment boundaries are located at the jump locations, and the value of each segment's constant simplicity level distinguishes heart sounds from murmurs. Unfortunately, the raw simplicity waveform acquired from sscope.filt_PCG is only an approximate PWC function that must be modified before it can be segmented in this way. This non-ideal behavior is primarily due to the intrinsic simplicity variation within sound segments but is also a result of the ripple generated from the sliding window that is used to obtain the waveform. Furthermore, the raw simplicity values in the silent segments or 
non-sound segments is often comparable to, or greater than, the simplicity values in the heart sound and murmur segments. This is a result of sscope.filt_PCG being reconstructed from a high level approximation coefficient (low frequency band), which has the effect of both attenuating and smoothing high frequency noise. Since the silent segments do not contain discernible sounds, the attenuated noise is the primary determinant of the simplicity in these segments. Even though the smoothing effect on the noise is hardly noticeable after filtering, the simplicity within the silent segments is nonetheless high because simplicity is amplitude-invariant (subplot-3 of Figure 4-10). Therefore, the simplicity within the non-sound segments is set to zero by peak peeling the PCG's fractal dimension and then zeroing the same samples in the simplicity waveform that are zero in the peak peeled fractal dimension (subplot-4 of Figure 4-10). This is similar to wavelet-based segmentation except that a second, constant threshold for removing low energy segments is unnecessary here. For efficiency, it is possible to avoid the zeroing operation (and therefore subplot-3) by only computing the simplicity waveform within the boundaries of the peaks acquired from peak peeling. The only reason this is not implemented here is to illustrate the non-ideal behavior of the simplicity waveform outside of the sound segments.

\subsubsection{Piecewise Constant Approximation}

After zeroing the silent segments' simplicity, the simplicity waveform must be converted to an ideal PWC function. For segments that only contain one sound, the average simplicity is sufficient for determining the simplicity level; but for segments that contain multiple sounds, a PWC approximation is required to determine the optimal jump locations and levels for each sound. Since this is a computationally intensive operation, and the non-sound segments have zero-value levels, a separate PWC approximation is performed on each segment rather than the entire waveform (subplot-5 of Figure 4-10). 
The L2 Potts minimization function from the MATLAB Pottslab toolbox is used to determine the optimal PWC approximation:

$$
P W C=\text { minL2Potts(signal, gamma) }
$$

The gamma constant here is proportional to the output's coarseness, where a small gamma closely tracks the input signal, and a large gamma ignores small deviations. The simplicity waveform's PWC approximation can be seen in subplot-5 of Figure 4-10, where the S1, S2, and the holosystolic murmurs that were merged in a single segment even after peak peeling (subplot-2) can now be individually segmented using the levels and jump locations in the PWC approximation. The steps required for transforming the raw simplicity waveform into an ideal PWC function are summarized below:

1. Calculate the PCG's fractal dimension

2. Peak peel the fractal dimension

3. Calculate the PCG's simplicity

4. Zero the simplicity in the silent segments

5. PWC approximate the simplicity within each segment 

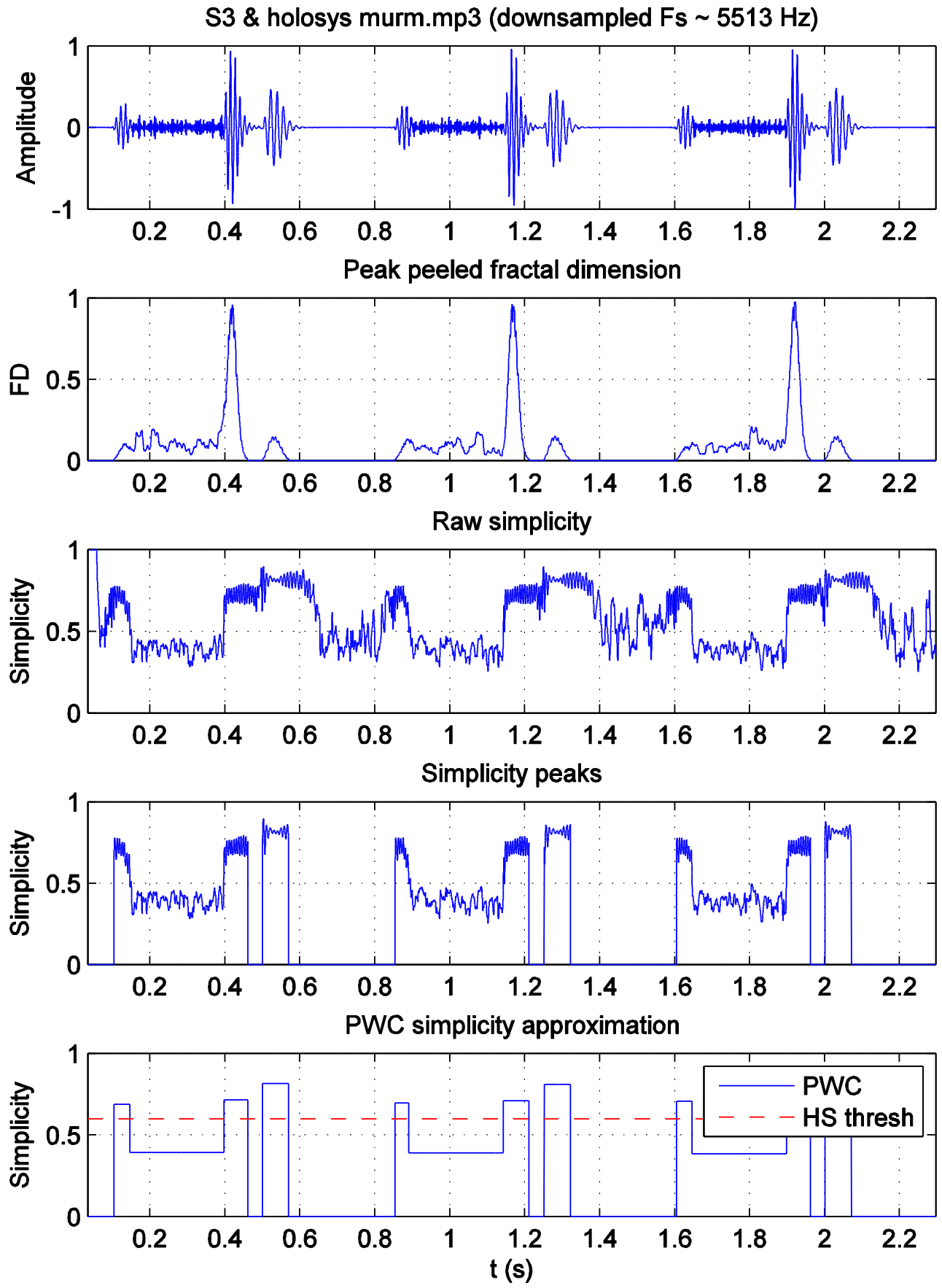

Figure 4-10: The peak peeled fractal dimension extracts the sound peaks from the background noise (subplot-2), which are used to zero the non-sound segments in the raw simplicity waveform (subplot-4) [chp4_seg.m]. 


\subsubsection{Heart Sound and Murmur Segmentation}

After obtaining the simplicity waveform's optimal PWC representation, the function:

$$
\text { seg = levels2seg(PWC) }
$$

generates a segment for each constant level situated between two jump locations, so that normal heart sounds, extra heart sounds, and murmurs can be identified by simple thresholding. The first threshold separates murmurs and heart sounds because murmurs have lower simplicity due to their complex and irregular shape, whereas heart sounds have higher simplicity due to their sinusoidal shape. Thus, unlike wavelet-based segmentation, heart sounds and murmurs are segmented here with a single waveform; and since the jump locations are the optimal segment boundaries, trim_HS() is not needed for removing murmur pieces from heart sounds. The second threshold separates normal and extra heart sounds, which is typically difficult through auscultation alone because both of these sound types are similar. Furthermore, extra heart sounds are often soft enough to be inaudible. As a result, the simplicity allows for the extra heart sounds S3, S4, and summation gallops to be properly identified when their simplicity levels are greater than those of normal heart sounds (including split sounds). In contrast, wavelet-based segmentation is incapable of distinguishing normal and extra heart sounds. Both thresholding operations can be seen in subplot-2 of Figure 4-11. 


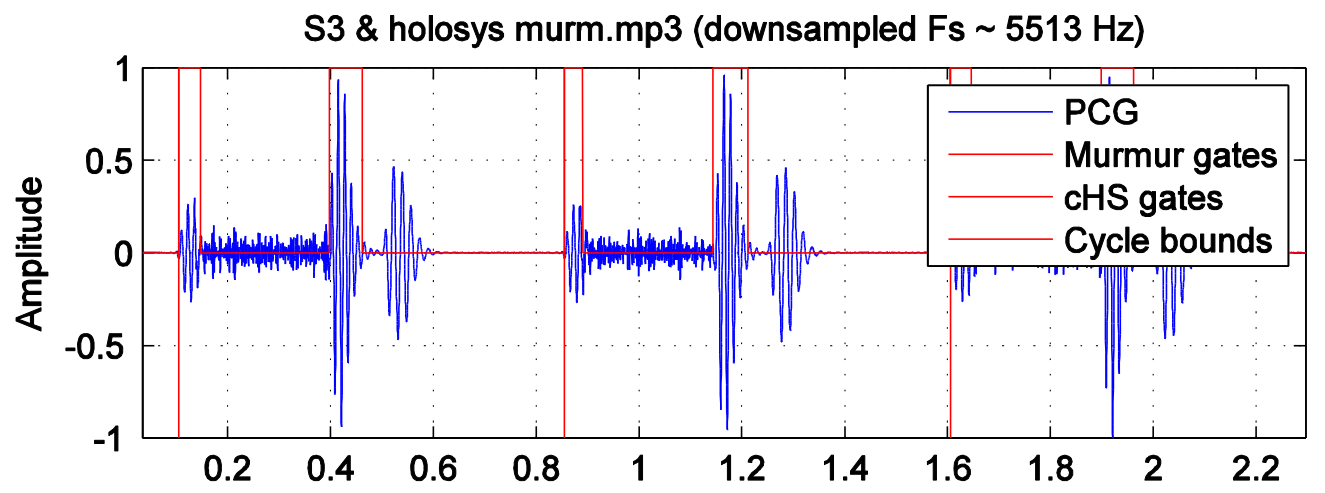

Threshold segments

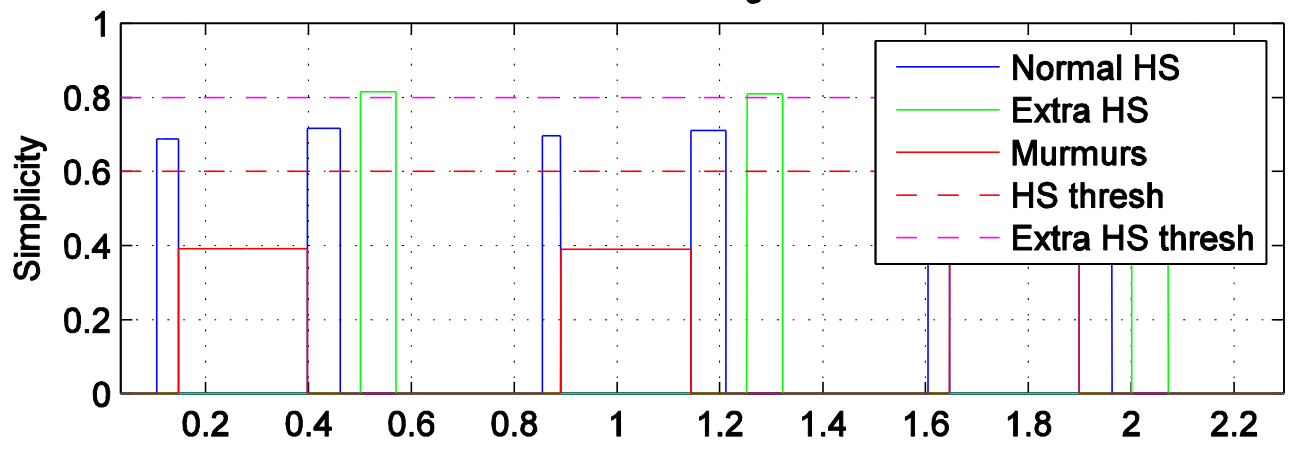

Separate split heart sounds

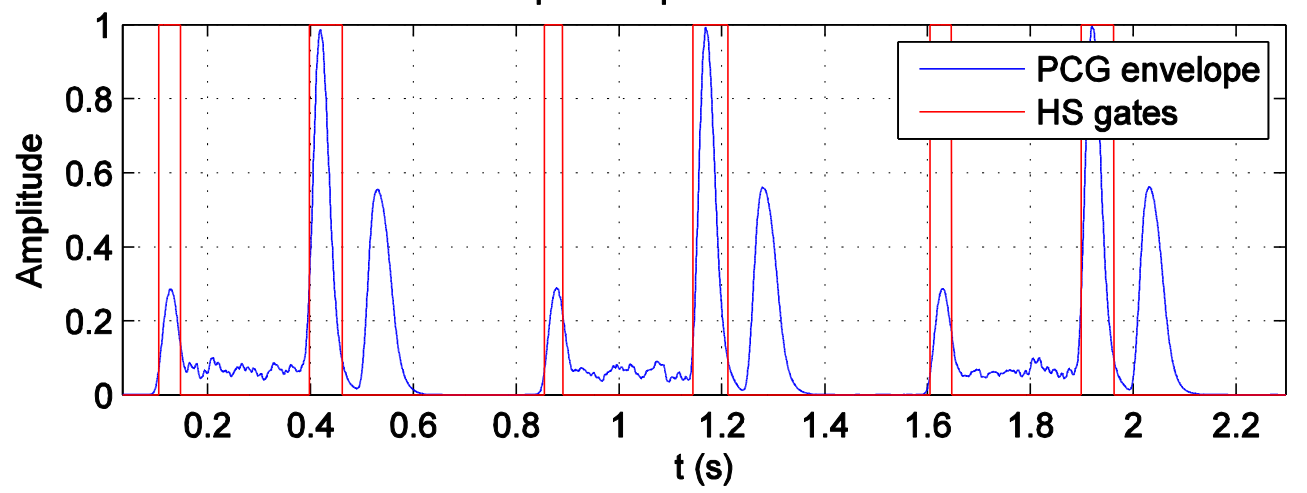

Figure 4-11: Threshold the normal heart sounds, extra heart sounds, and murmur segments by their simplicity levels (subplot-2) [chp4_seg.m].

\subsubsection{Split Sound Detection, Heart Cycle Segmentation, and Sound Segment Classification}

The methods used to segment normal heart sounds, extra heart sounds, and murmurs are unique to each segmentation function, but the remaining steps required to 
fully classify the segments are nearly identical regardless of the chosen technique. The first of these is separating heart sound segments that contain split sounds by applying split_HS() to the PCG's envelope. The heart cycle boundaries are then obtained with find_heart_cycles(), and the normal heart sound segments (excluding split sounds) are restricted to two segments per cycle with limit_HS(). Finally, the normal heart sounds, extra heart sounds, and murmurs are classified and stored in segments arrays corresponding to specific sound types with Ibl_sounds(). 


\section{Segmentation Results}

\subsection{Introduction}

\subsubsection{Sound File Datasets}

The sound files used for testing the segmentation functions are taken from two common auscultation training datasets: the Littmann [33] and the University of Michigan [34] heart sound libraries.

\subsubsection{Segmentation Errors and Detection Rates}

The primary purpose of PCG segmentation is to detect murmurs because their presence indicates an unhealthy heart. Normal heart sounds must also be detected because they are used to locate the heart cycle boundaries and to classify the sound segment types within each heart cycle. Thus, faulty segmentation is primarily caused by the incorrect detection of normal heart sounds and murmurs. In particular, this can occur when heart sounds are misidentified as murmurs and vice versa, or when heart sounds and murmurs are not detected at all. The detection of split heart sounds and extra heart sounds is also desirable but not an absolute requirement for diagnosing heart health, as it is often challenging even through auscultation to distinguish extra sounds from split sound components. Therefore, successful segmentation here only requires the proper detection of normal heart sounds and murmurs.

The performance of the wavelet and simplicity-based segmentation methods, as it relates to medical diagnosis, is evaluated with the false negative detection rate (FNDR) and the false positive detection rate (FPDR). A false negative occurs when no murmurs are detected in a murmur-containing heart cycle, which is typically caused by murmurs being misidentified as heart sounds (false heart sounds). Conversely, a false positive occurs when a murmur is detected in a murmur-free heart cycle, which is caused by a 
heart sound being misidentified as a murmur (false murmur). The false negative and false positive detection rates are defined below:

FNDR = \# of murmur-containing heart cycles without detected murmurs / total \# of murmur-containing heart cycles

FPDR $=\#$ of murmur-free heart cycles with detected false murmurs / total \# of murmur-free heart cycles

These two metrics indicate the effectiveness of murmur detection by the two segmentation methods when considering a complete heart sound cycle (composed of one systole and one diastole segment) as the fundamental unit in which murmurs should be detected if present. This is similar to when a medical clinician evaluates heart function during auscultation, as the primary focus is on determining whether or not a murmur is detected in each heart cycle.

The FNDR and FPDR are sufficient statistics for clinicians, but the true murmur detection rate (TMDR) and false murmur detection rate (FMDR) are also necessary to fully evaluate the performance of the two methods. These rates are defined below:

TMDR = \# of detected true murmurs / total \# of murmurs

FMDR = \# of heart cycles with detected false murmurs / total \# of heart cycles

For the TMDR, the murmurs are defined as either murmur-containing systole or diastole segments, so even multiple murmur segments within a systole or diastole segment are counted as a single murmur. Therefore, the true murmurs are defined as the number of properly detected and classified murmur-containing systole or diastole segments rather than individual murmur segments. For the FMDR, the heart cycles include both the 
murmur-free and murmur-containing heart cycles, so it is a more general rate than the FPDR, which only considers the murmur-free heart cycles.

Even though the TMDR and FNDR have different evaluative purposes, they are inversely related. This is because an increase in the number of detected true murmurs tends to decrease the number of false negative heart cycles. For example, if each heart cycle contains a systolic murmur, then each additional detected systolic murmur increases the TMDR and decreases the FNDR.

The underlying causes for the false positives, false negatives, and false murmurs are discussed and illustrated with examples in sections 5.2 and 5.3.

\subsubsection{False Negative and False Positive Detection Rates}

The dataset sound files are separated into those that do and do not contain murmurs. Since false negatives can only occur in murmur-containing cycles, and false positives can only occur in murmur-free cycles, the FNDR only applies to the sound files that contain murmurs, and the FPDR only applies to the sound files that do not contain murmurs.

For both wavelet and simplicity-based segmentation, the false negatives are enumerated in Table 5-1 (Michigan) and Table 5-3 (Littmann), and the false positives are enumerated in Table 5-2 (Michigan) and Table 5-4 (Littmann). All heart cycles in the false negative tables contain murmurs, while none of the heart cycles in the false positive tables contain murmurs. The false negative and false positive data is then used to determine the FNDR in Table 5-5 and the FPDR in Table 5-6, and both of these detection rates are compared in Table 5-7. 
Table 5-1: False negatives (Michigan).

\begin{tabular}{|l|c|c|c|}
\hline \multirow{2}{*}{ Filename } & $\begin{array}{c}\text { Murmur-containing } \\
\text { cycles }\end{array}$ & \multicolumn{2}{|c|}{$\begin{array}{c}\text { Murmur-containing cycles } \\
\text { without detected murmurs }\end{array}$} \\
\cline { 2 - 4 } & 5 & Wavelet & Simplicity \\
\hline early dias murm & 4 & 2 & 5 \\
\hline $\begin{array}{l}\text { ejection click \& syst } \\
\text { eject murm \& single S2 }\end{array}$ & 5 & 0 & 0 \\
\hline mid sys click & 5 & 0 & 0 \\
\hline OS \& dias murm & 6 & 0 & 1 \\
\hline S3 \& holosys murm & 5 & 1 & 0 \\
\hline S4 \& mid sys murm & 5 & 0 & 0 \\
\hline $\begin{array}{l}\text { sys click \& late sys } \\
\text { murm }\end{array}$ & 6 & 0 & 0 \\
\hline sys murm \& absent S2 & 6 & 0 & 0 \\
\hline sys \& dias murm & 6 & 0 & 0 \\
\hline $\begin{array}{l}\text { sys eject murm \& split } \\
\text { S2 trans }\end{array}$ & 5 & 0 & 0 \\
\hline $\begin{array}{l}\text { sys eject murm \& split } \\
\text { S2 pers }\end{array}$ & 58 & 0 & 0 \\
\hline Total & & 3 & 6 \\
\hline
\end{tabular}

Table 5-2: False positives (Michigan).

\begin{tabular}{|l|c|c|c|}
\hline \multirow{2}{*}{ Filename } & \multirow{2}{*}{ Murmur-free cycles } & \multicolumn{2}{|c|}{$\begin{array}{c}\text { Murmur-free cycles with detected } \\
\text { false murmurs }\end{array}$} \\
\cline { 3 - 4 } & & Wavelet & Simplicity \\
\cline { 3 - 4 } & & 0 & 0 \\
\hline normal 1 & 5 & 4 & 0 \\
\hline normal 2 & 5 & 5 & 0 \\
\hline S3 & 5 & 5 & 0 \\
\hline S4 & 5 & 0 & 0 \\
\hline single S2 & 5 & 0 & 0 \\
\hline split S1 pers & 6 & 0 & 0 \\
\hline split S2 pers & 6 & 0 & 0 \\
\hline split S2 trans & 6 & 14 & 0 \\
\hline Total & 43 & & \\
\hline
\end{tabular}


Table 5-3: False negatives (Littmann).

\begin{tabular}{|l|c|c|c|}
\hline \multirow{2}{*}{ File Name } & $\begin{array}{c}\text { Murmur-containing } \\
\text { cycles }\end{array}$ & \multicolumn{2}{c|}{$\begin{array}{c}\text { Murmur-containing cycles } \\
\text { without detected murmurs }\end{array}$} \\
\cline { 2 - 4 } & & Wavelet & Simplicity \\
\hline AP & 0 & 0 & 0 \\
\hline$A R$ & 4 & 0 & 0 \\
\hline AS & 3 & 0 & 0 \\
\hline ASD & 2 & 0 & 0 \\
\hline COA & 2 & 0 & 0 \\
\hline EA & 3 & 0 & 0 \\
\hline eject click & 2 & 0 & 0 \\
\hline eject click \& AS & 2 & 0 & 0 \\
moderate \& AR mild & 2 & 0 & 0 \\
\hline innocent murmur & 4 & 0 & 0 \\
\hline late sys click & 3 & 0 & 0 \\
\hline mid sys click & 2 & 0 & 0 \\
\hline MR severe & 3 & 1 & 3 \\
\hline MS moderate & 4 & 0 & 0 \\
\hline MVP & 2 & 0 & 0 \\
\hline OS & 2 & 0 & 0 \\
\hline PDA & 2 & 0 & 0 \\
\hline S4 \& AS severe & 2 & 0 & 0 \\
\hline TR severe & 2 & 0 & 0 \\
\hline VSD & 46 & 1 & 3 \\
\hline Total & & & \\
\hline & & 0 & 0 \\
\hline
\end{tabular}

Table 5-4: False positives (Littmann).

\begin{tabular}{|l|c|c|c|}
\hline \multirow{2}{*}{ Filename } & \multirow{2}{*}{ Murmur-free cycles } & \multicolumn{2}{c|}{$\begin{array}{c}\text { Murmur-free cycles with } \\
\text { detected false murmurs }\end{array}$} \\
\cline { 3 - 4 } & & Wavelet & Simplicity \\
\hline normal & 2 & 0 & 0 \\
\hline S3 \& S4 & 3 & 0 & 0 \\
\hline S3 abnormal & 3 & 0 & 0 \\
\hline S3 physio & 2 & 0 & 0 \\
\hline S4 & 2 & 0 & 0 \\
\hline sum gallop & 6 & 2 & 0 \\
\hline split S2 fixed & 4 & 0 & 0 \\
\hline split S2 physio & 4 & 0 & 0 \\
\hline split S1 & 4 & 0 & 0 \\
\hline Total & 30 & 2 & 0 \\
\hline
\end{tabular}


Table 5-5: False negative detection rates (FNDR).

\begin{tabular}{|l|c|c|c|c|c|}
\hline \multirow{2}{*}{ Dataset } & \multirow{2}{*}{$\begin{array}{c}\text { Murmur- } \\
\text { containing } \\
\text { cycles }\end{array}$} & $\begin{array}{c}\text { Murmur-containing } \\
\text { cycles without } \\
\text { detected murmurs }\end{array}$ & \multicolumn{2}{|c|}{ FNDR } \\
\cline { 3 - 6 } & & Wavelet & Simplicity & Wavelet & Simplicity \\
\hline Michigan & 58 & 3 & 6 & $5 \%$ & $10 \%$ \\
\hline Littmann & 46 & 1 & 3 & $2 \%$ & $7 \%$ \\
\hline Total & 104 & 4 & 9 & $4 \%$ & $9 \%$ \\
\hline
\end{tabular}

Table 5-6: False positive detection rates (FPDR).

\begin{tabular}{|l|c|c|c|c|c|}
\hline \multirow{2}{*}{ Dataset } & \multirow{2}{*}{$\begin{array}{c}\text { Murmur-free } \\
\text { Cycles }\end{array}$} & $\begin{array}{c}\text { Murmur-free cycles } \\
\text { with detected false } \\
\text { murmurs }\end{array}$ & \multicolumn{2}{|c|}{ FPDR } \\
\cline { 3 - 6 } & & Wavelet & Simplicity & Wavelet & Simplicity \\
\hline Michigan & 43 & 14 & 0 & $33 \%$ & $0 \%$ \\
\hline Littmann & 30 & 2 & 0 & $7 \%$ & $0 \%$ \\
\hline Total & 73 & 16 & 0 & $22 \%$ & $0 \%$ \\
\hline
\end{tabular}

Table 5-7: FNDR and FPDR comparison for wavelet and simplicity-based segmentation.

\begin{tabular}{|l|c|c|}
\hline Technique & FNDR & FPDR \\
\hline Wavelet & $4 \%$ & $22 \%$ \\
\hline Simplicity & $9 \%$ & $0 \%$ \\
\hline
\end{tabular}

\subsection{Wavelet-Based Segmentation}

\subsubsection{Wavelet Constants}

The constants for wavelet-based segmentation, which consist of window lengths, peak peeling stopping conditions, thresholds, and wavelet filter-specific values, are listed in Table 5-8. All constants can be changed from their defaults using name-value pair arguments passed to dwt_segment() (except for $I V I$, which is an optional positional argument). Also, the HS_thresh constant has two values listed for each dataset: 0.1 for the Michigan dataset (default value) and 0.05 for the Littmann dataset. 
Table 5-8: Wavelet constants.

\begin{tabular}{|c|c|c|}
\hline Constant & Value & Description \\
\hline$|V|$ & 5 & $\begin{array}{l}\text { Wavelet decomposition level for attenuating murmurs in } \\
\text { the PCG }\end{array}$ \\
\hline wavef & 'db6’ & Wavelet function \\
\hline w & $20 \mathrm{~ms}$ & Fractal dimension and energy waveform window lengths \\
\hline STCW & $10^{-4}$ & $\begin{array}{l}\text { Peak peeling stopping condition for the wavelet-filtered } \\
\text { PCG }\end{array}$ \\
\hline HS_thresh & $\begin{array}{l}0.1 \\
0.05\end{array}$ & Minimum allowable energy for heart sound segments \\
\hline WS & $20 \mathrm{~ms}$ & PCG smoothing window length \\
\hline max_tr & 0.5 & $\begin{array}{l}\text { rel_thresh argument for trim_HS() that specifies the } \\
\text { segment thresholds as a fraction of their maximum } \\
\text { amplitudes }\end{array}$ \\
\hline min_pk & 0.2 & $\begin{array}{l}\text { min height argument for split_HS() that specifies the } \\
\text { minimum peak height for a split sound component }\end{array}$ \\
\hline STCF & $10^{-4}$ & $\begin{array}{l}\text { Peak peeling stopping condition for the fractal dimension } \\
\text { waveform }\end{array}$ \\
\hline
\end{tabular}

\subsubsection{Wavelet Errors}

The most common wavelet-based segmentation errors occur while thresholding the energy waveform of the wavelet-filtered PCG. Heart sound segments, including normal, extra, and split sounds, are mistaken for murmurs when their maximum energies are less than the energy threshold, while murmur segments are mistaken for heart sounds when their maximum energies are greater than the energy threshold.

Figure 5-1 and Figure 5-2 illustrate how a normal heart sound can be misidentified as a murmur when its maximum energy is less than the energy threshold, occurring in a sound file with an extra S3 sound. As can be seen in subplot-3 of Figure 5-1, the first S1 segment's maximum energy is less than the threshold, whereas the second S1 segment's maximum energy is slightly greater than the threshold. Thus, the first $\mathrm{S} 1$ is misidentified 
as a murmur, but the second $S 1$ is properly identified (subplot-4 of Figure 5-2). As a result, the first heart cycle's start boundary is placed on the start index of the S2 instead of the S1 segment, which shortens the heart cycle's duration, removes the systolic murmur from the heart cycle, and causes S2 to be erroneously classified as S1 (subplot-1 of Figure 5-2). In addition, the S2 and S3 segments in both cycles are misidentified as split components due to their close proximities and the lack of extra heart sound discrimination for wavelet-based segmentation. In particular, the S2 and S3 segments in the first cycle are considered split S1 components, but the S2 and S3 segments in the second cycle are more appropriately considered split S2 components. Classifying extra heart sounds as split components is not considered an error per se because the extra sounds resemble split sounds and are typically located near S1 or S2. Therefore, only a split S2 is detected in the first cycle as opposed to the S1, systolic murmur, and split S2 detected in the second cycle. 

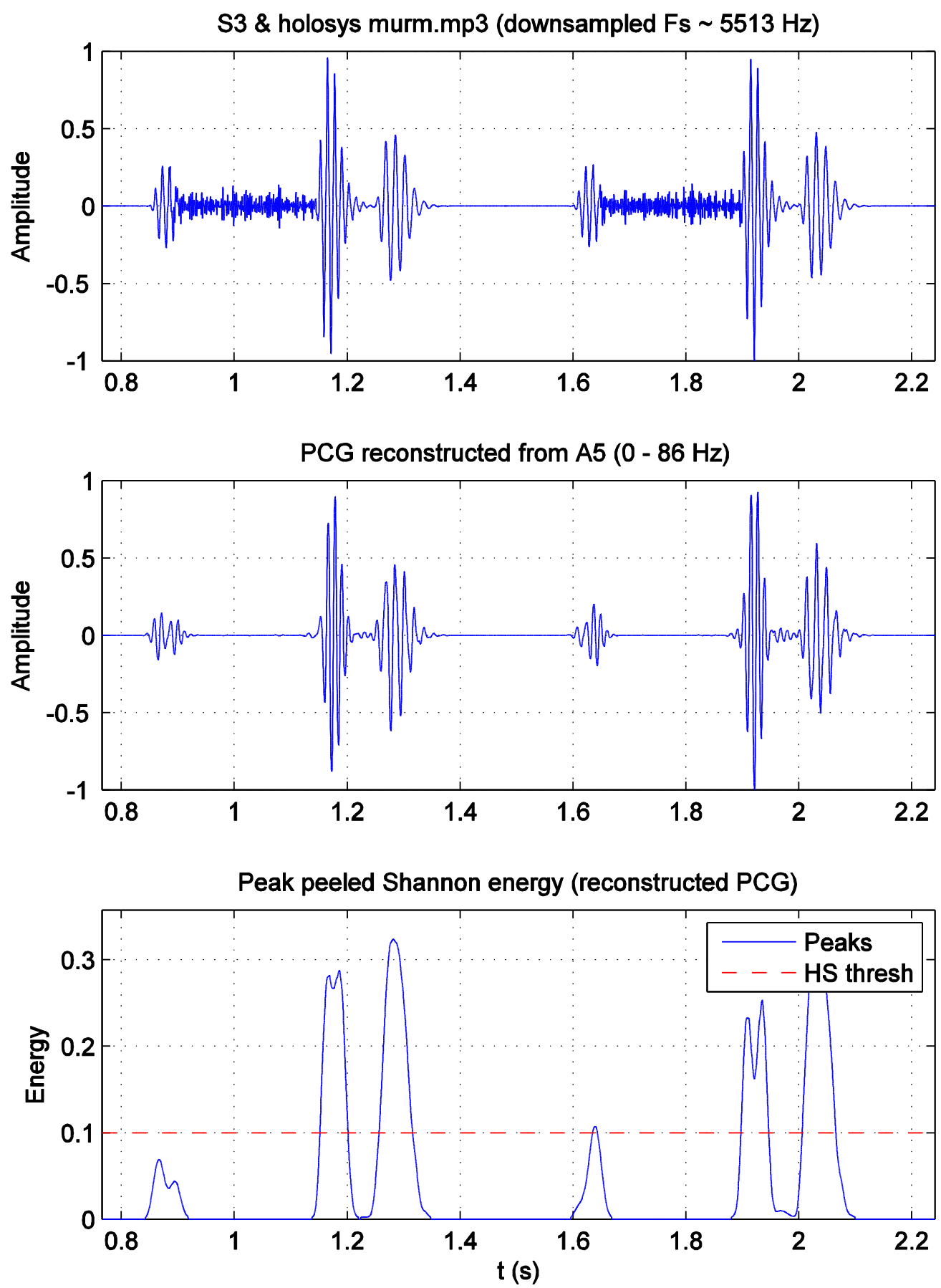

Figure 5-1: The first $\mathrm{S} 1$ is mistaken for a murmur because its maximum energy is less than the energy threshold (subplot-3) [dwt_michigan.m]. 


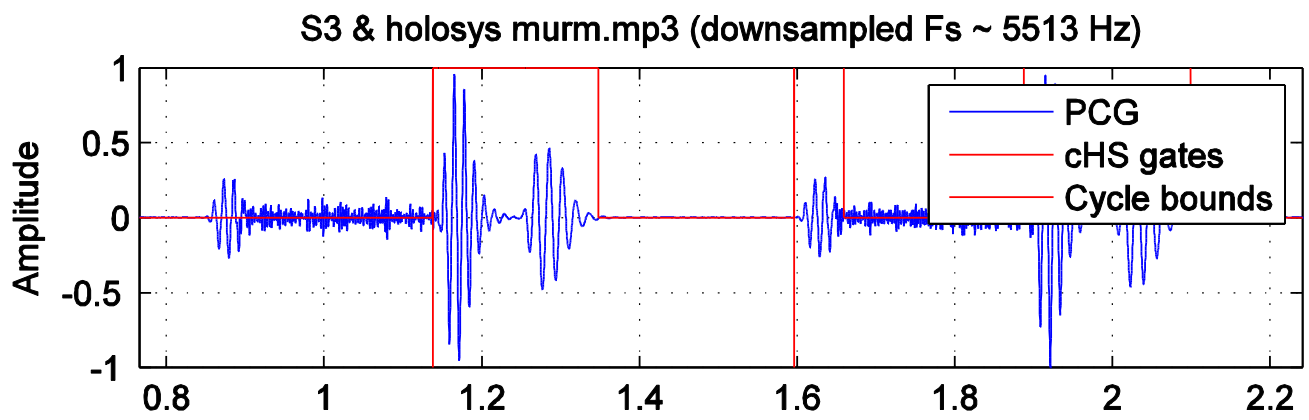

Trim segments

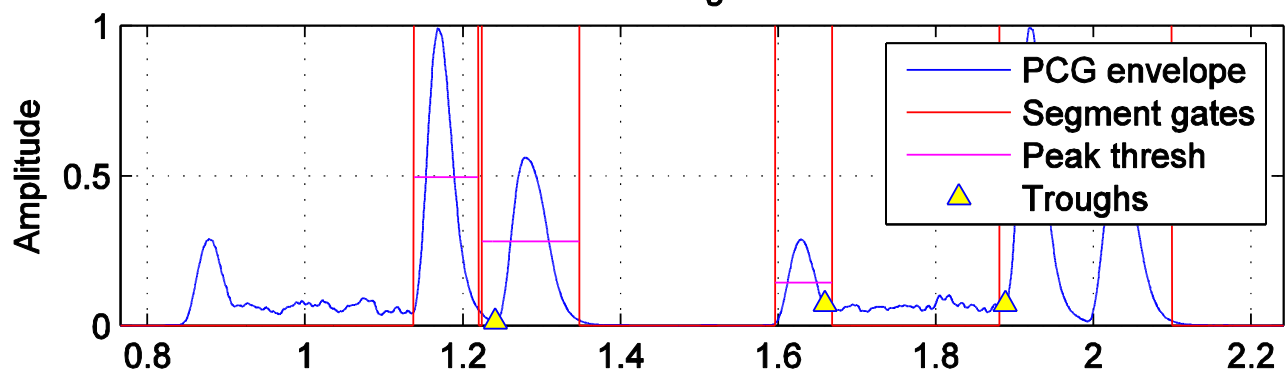

Separate split heart sounds
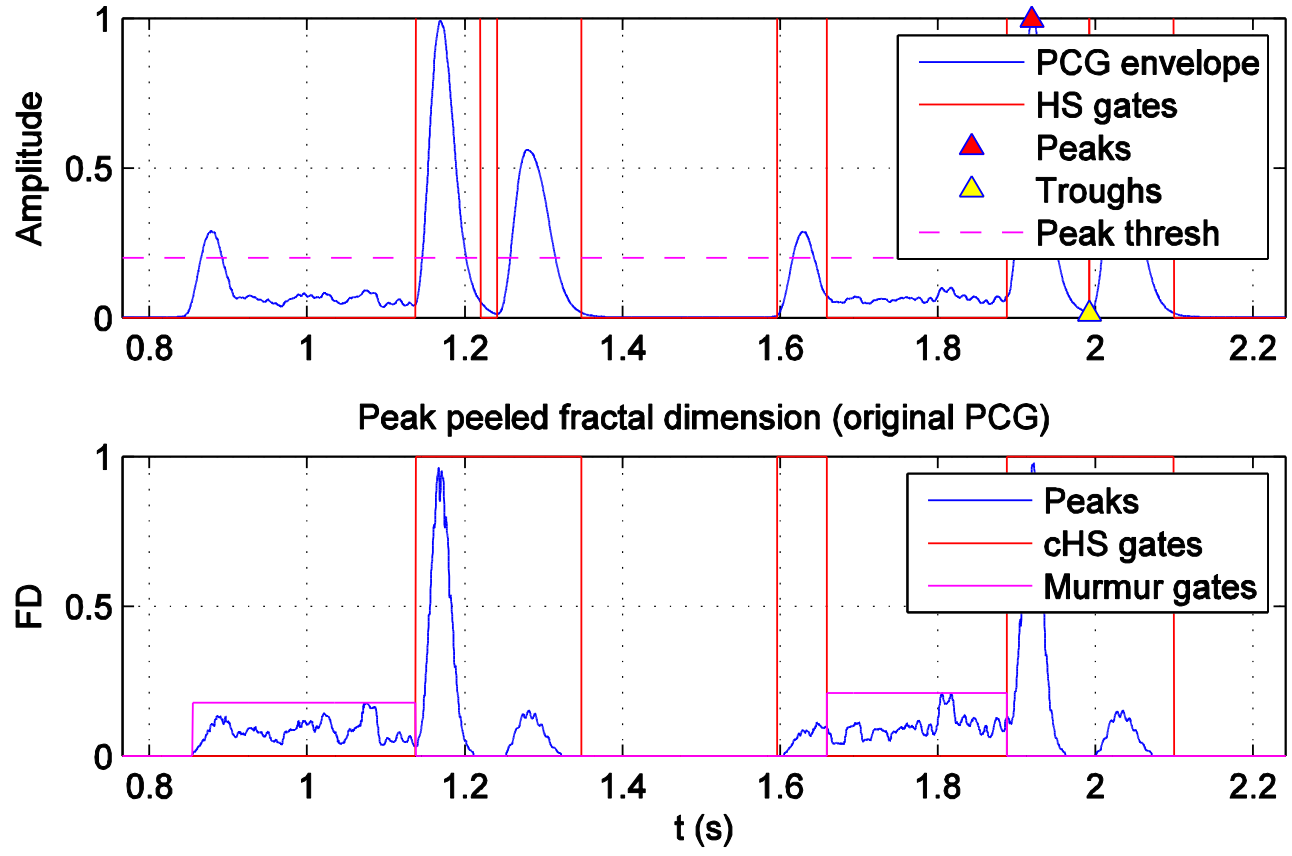

Figure 5-2: The first $\mathrm{S} 1$ segment is misidentified as a murmur, but the second $\mathrm{S} 1$ segment is properly identified (subplot-4). As a result, the first heart cycle's start boundary is moved from S1 to the nearest S2 (subplot-1) [dwt_michigan.m]. 
Figure 5-3 and Figure 5-4 illustrate how an S4 segment can be misidentified as a murmur when its maximum energy is less than the heart sound threshold. As can be seen in subplot-3 of Figure 5-3, similar to the previous example, the first S4 segment's maximum energy is less than the threshold, while the second S4 segment's maximum energy is greater than the threshold. As a result, the first $\mathrm{S4}$ is misidentified as a murmur, but the second S4 is acceptably classified as a split S1 component give its proximity to S1 (subplot-4 of Figure 5-4). However, unlike the previous example, the cycle boundaries here are correctly located since neither S1 nor S2 is removed during segmentation (subplot-1 of Figure 5-4). 

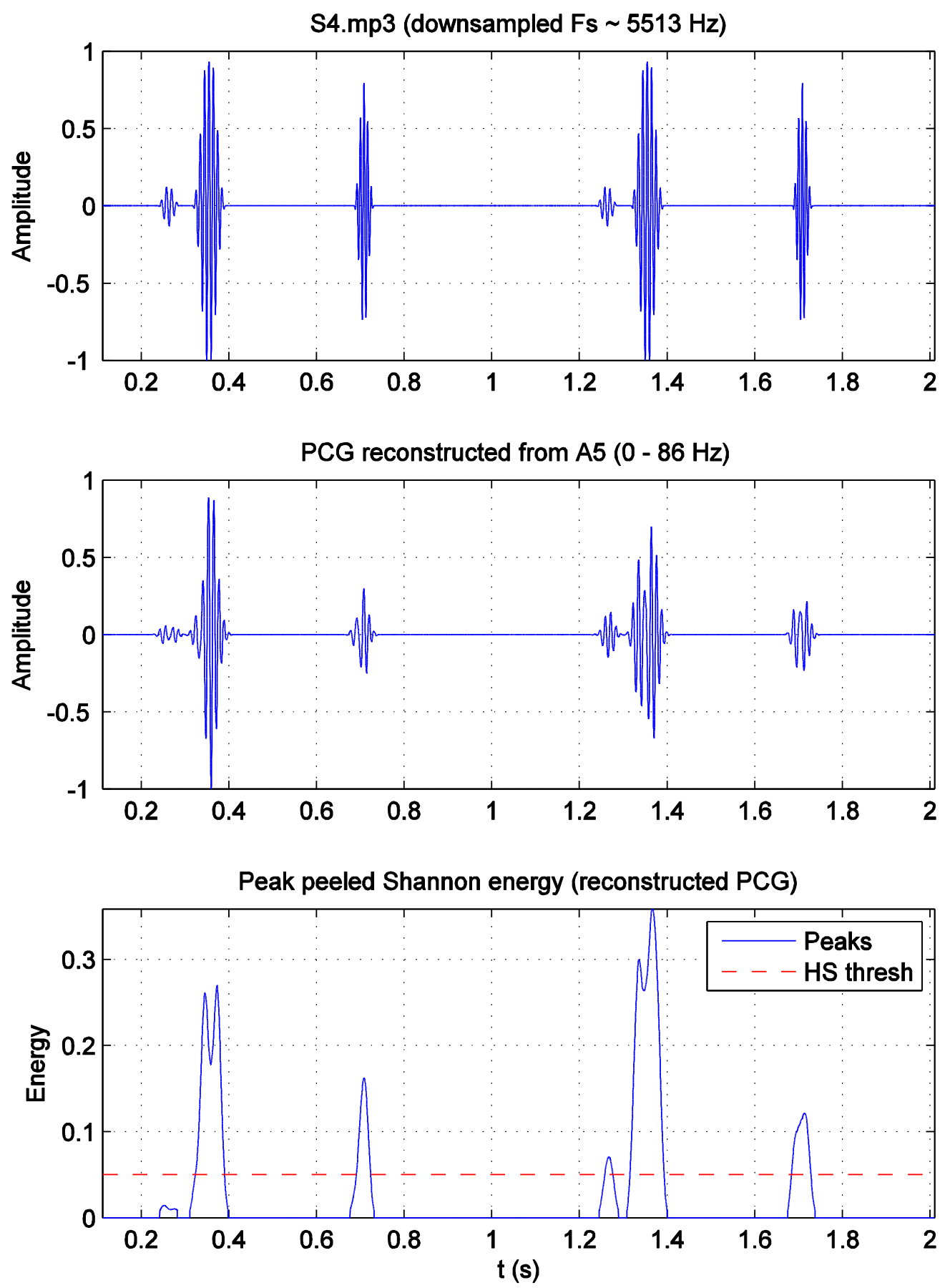

Figure 5-3: The first S4 is misidentified as a murmur because its maximum energy is less than the energy threshold (subplot-3) [dwt_littmann.m]. 
S4.mp3 (downsampled Fs $5513 \mathrm{~Hz}$ )

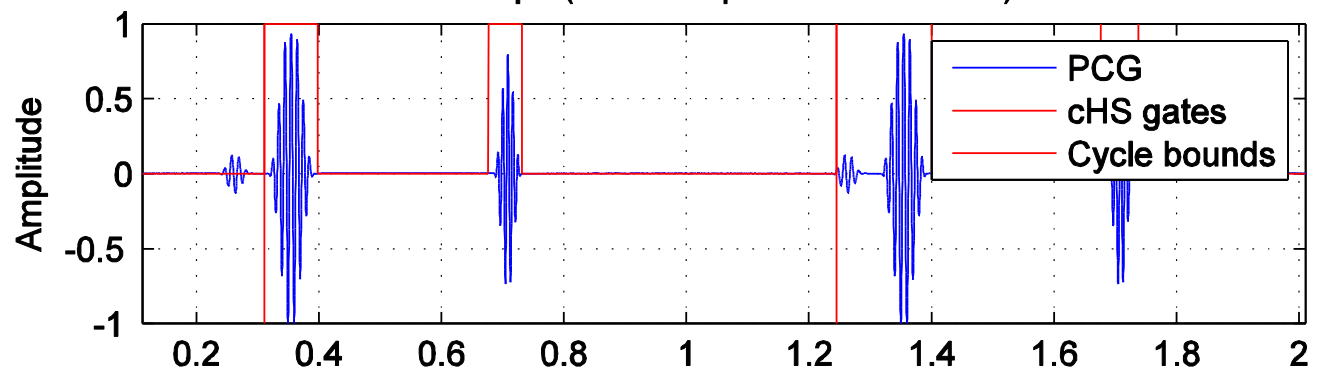

Trim segments

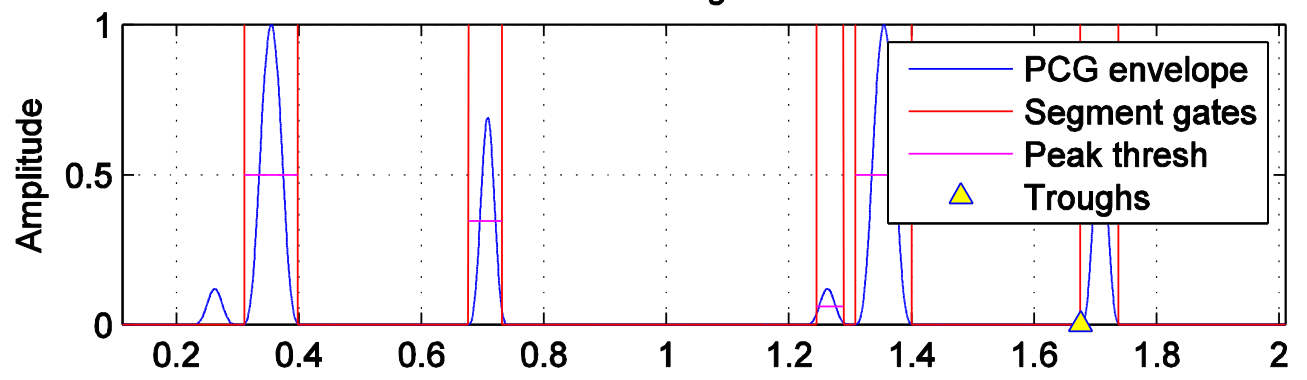

Separate split heart sounds

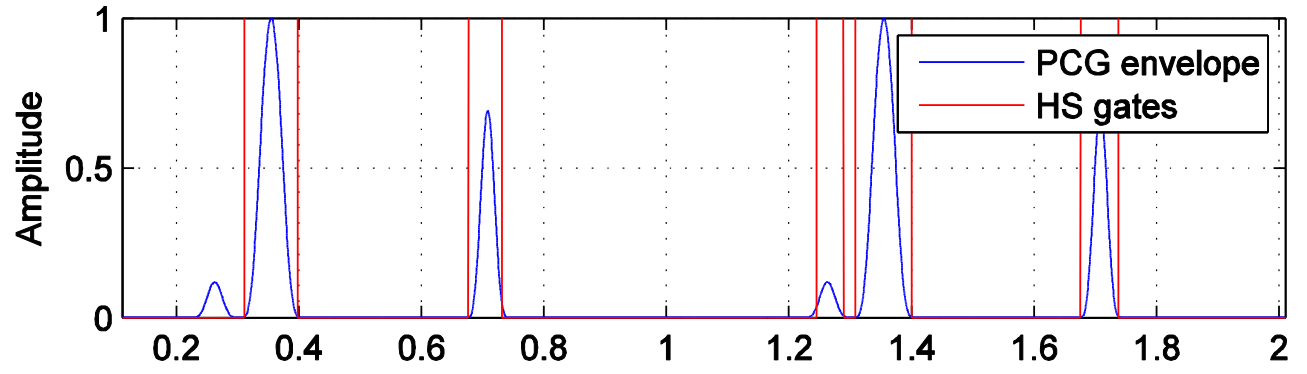

Peak peeled fractal dimension (original PCG)

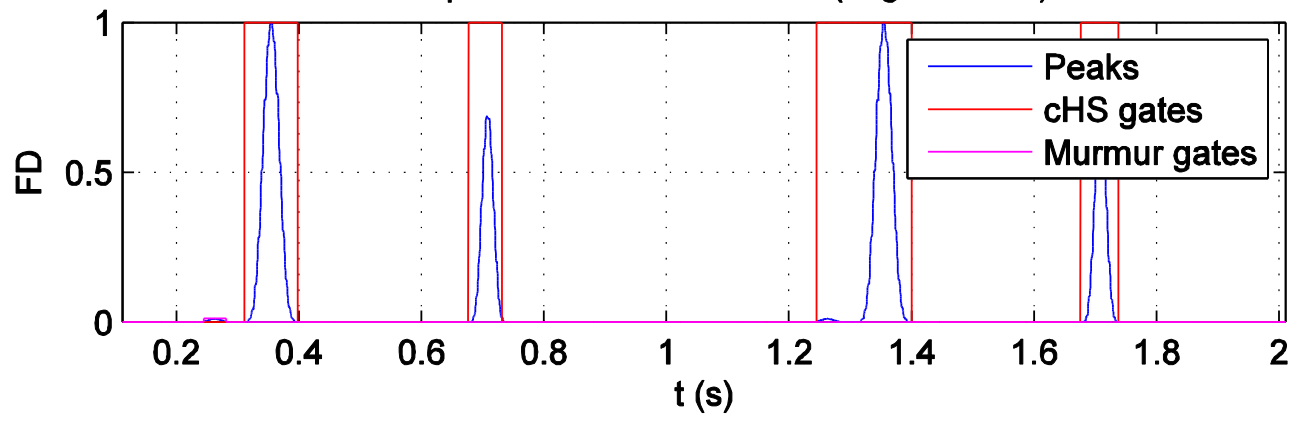

Figure 5-4: The first S4 is misidentified as a murmur, but the second S4 is acceptably misidentified as a split sound component (subplot-4). The cycle boundary locations are correct because S1 and S2 are properly identified (subplot-1) [dwt_littmann.m]. 
Figure 5-5 illustrates how a murmur can be misidentified as a heart sound when its maximum energy is greater than the heart sound threshold. The first heart cycle in this example contains both a systolic and a diastolic murmur (subplot-1). The high-frequency systolic murmurs in the first and second cycles are attenuated sufficiently after wavelet filtering, but the diastolic murmur in the first cycle is largely unaffected by filtering due to its low frequency content and its resemblance of a heart sound (subplot-2). As a result, the diastolic murmur is misidentified as a heart sound because its maximum energy is greater than the threshold (subplot-3). 

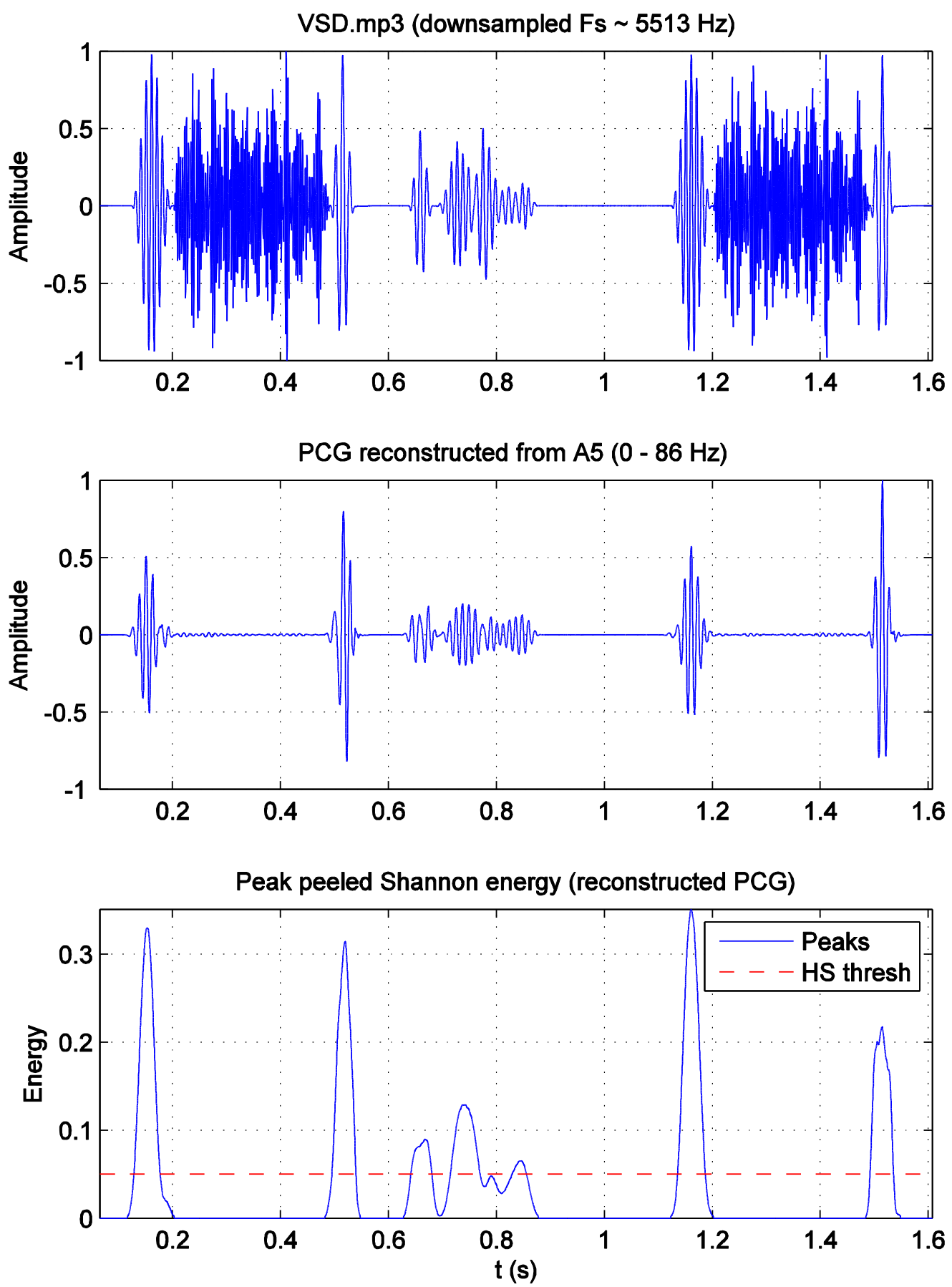

Figure 5-5: The diastolic murmur is misidentified as a heart sound because its maximum energy is greater than the threshold (subplot-3) [dwt_littmann.m]. 
The function trim_HS() is a common source of errors for wavelet-based segmentation due to its reliance on thresholding the PCG's envelope to remove murmur samples from the heart sound segments. Erroneous results are caused by three situations in particular. The first error occurs when two heart sounds reside in a single segment, and trim_HS() repositions the segment boundaries to remove one of the sounds because its peak is below the segment's threshold. As a result, the heart sound is misidentified as a murmur. The second error occurs when a murmur and a heart sound reside in the same segment, but the murmur is not removed because its peak is above the segment's threshold. As a result, the murmur is misidentified as a split sound component. Finally, the third error occurs when a heart sound segment's boundaries are repositioned by trim_HS() despite the lack of murmurs in the segment. This is typically caused by a disturbance in the heart sound that manifests as a small trough in the PCG's envelope, which is mistaken for a junction between a heart sound and a murmur.

Figure 5-6 illustrates the first type of error caused by trim_HS(). Here, each S4 and S1 pair resides in a single heart sound segment because they were not separated during peak peeling. Since the S4 peaks are below their respective segment thresholds, trim_HS() removes S4 from the heart sound segments (subplot-2). After the segment boundaries are repositioned, the $\mathrm{S} 4$ are misidentified as murmurs (subplot-4). 


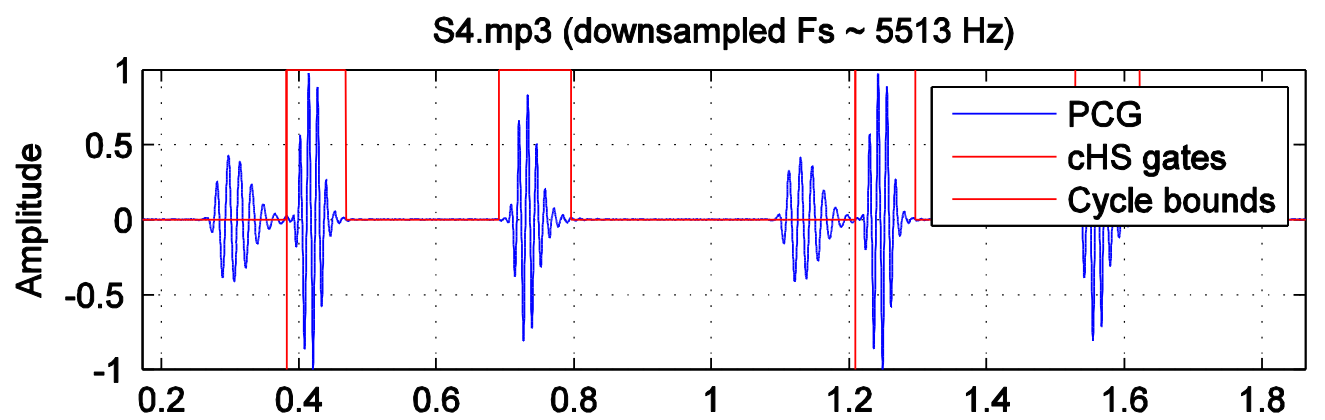

Trim segments
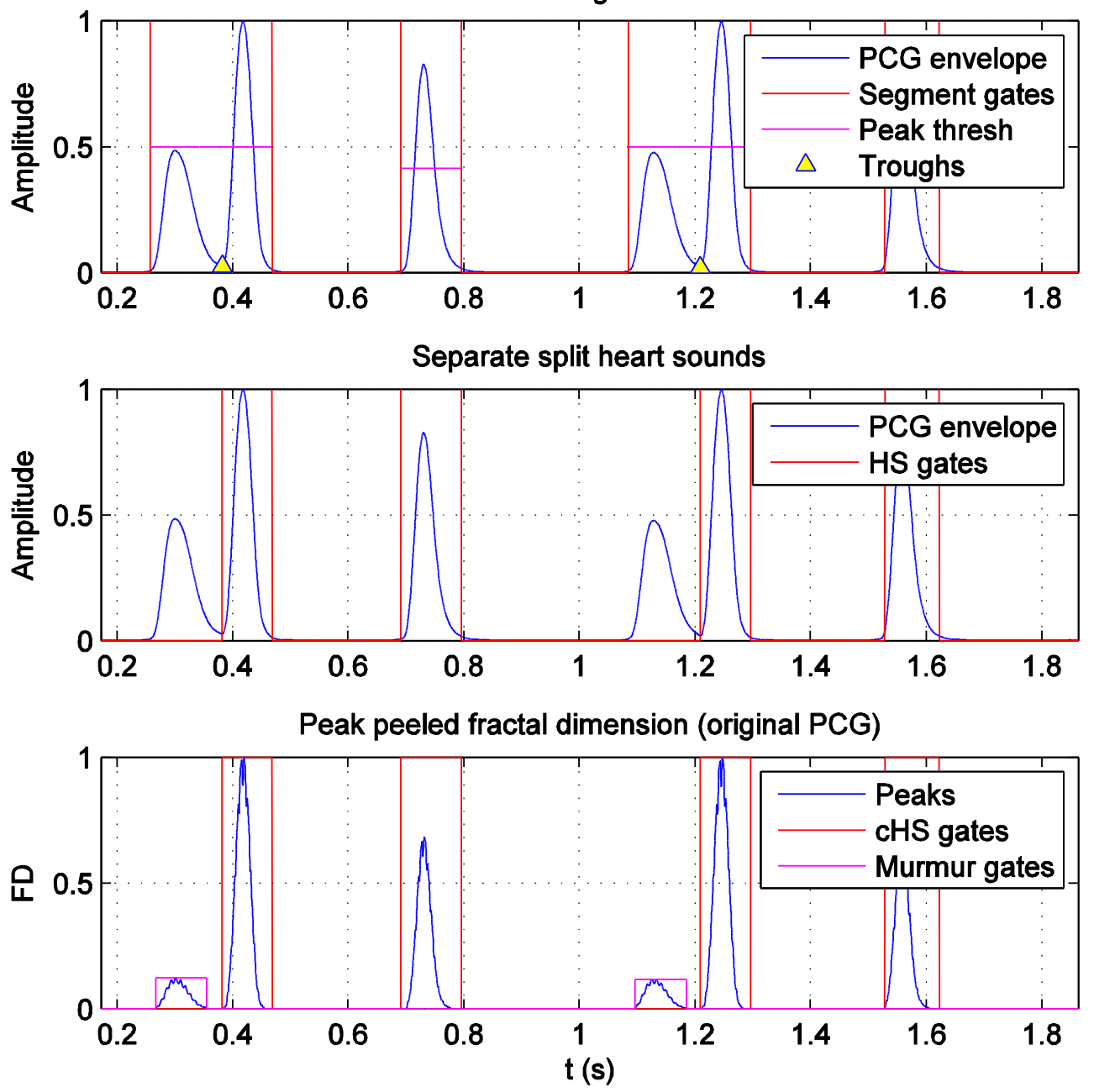

Figure 5-6: The S4 peaks are below the segment thresholds (subplot-2) and are therefore misidentified as murmurs (subplot-4) [dwt_michigan.m]. 
Figure 5-7 illustrates the second and third type of error caused by trim_HS(). In the first segment of the first heart cycle, which contains an S2 and an opening snap murmur, the murmur is exempt from removal because its peak is above the threshold (subplot-2). As a result, the murmur is misidentified as a split $\mathrm{S} 2$ component (subplot-3). In the second segment of the first cycle, which contains an S1 and a late diastolic murmur, the murmur is successfully removed because trim_HS() repositions the segment's left boundary (subplot-2). However, the segment's right boundary is also repositioned even though a systolic murmur does not exist within that segment. As a result, the portion of $\mathrm{S} 1$ that is removed is misidentified as a murmur (subplot-4). 


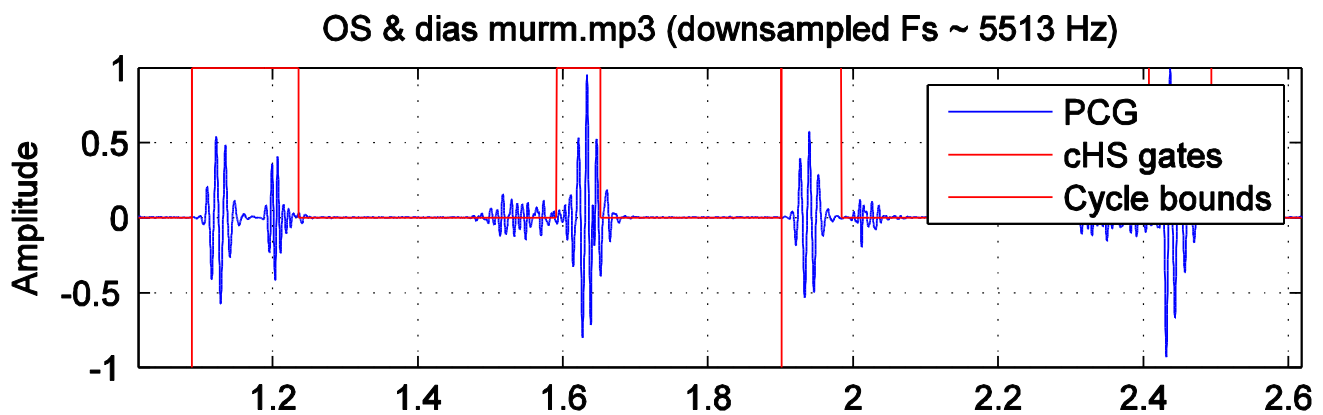

Trim segments

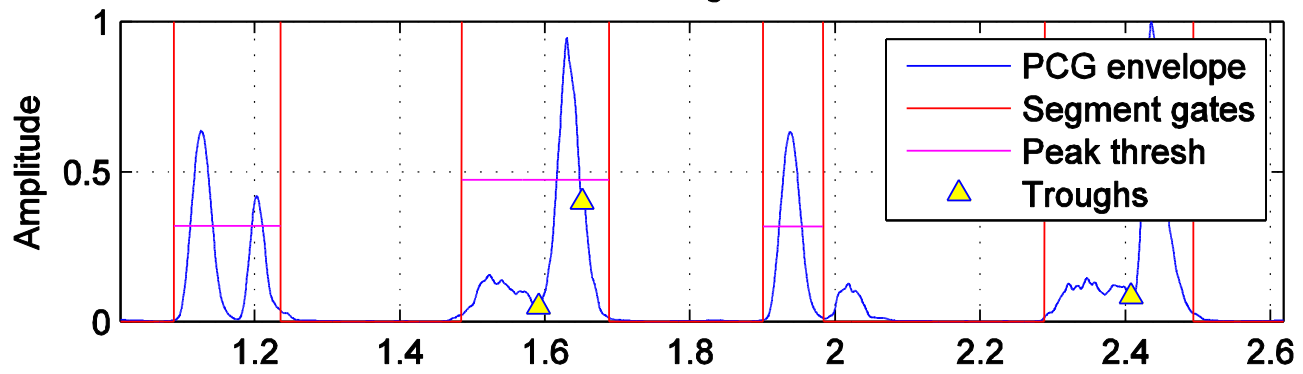

Separate split heart sounds

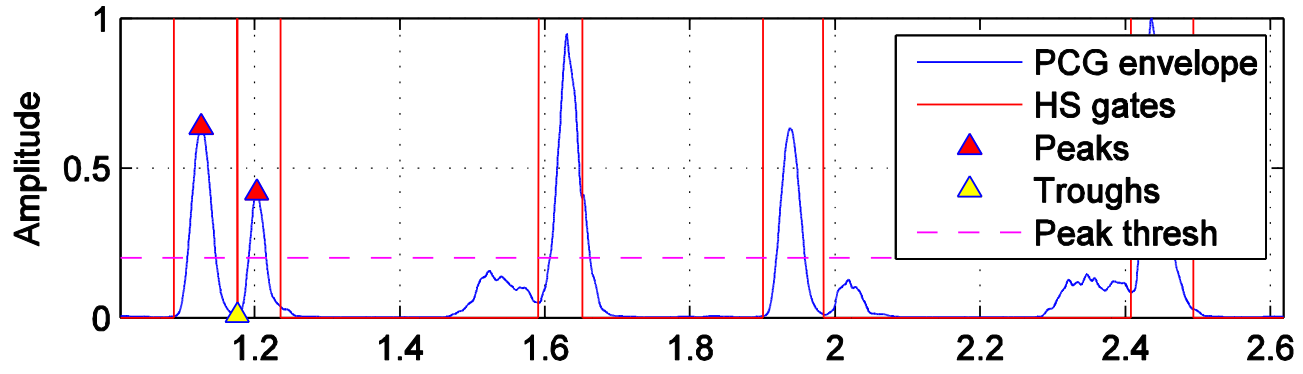

Peak peeled fractal dimension (original PCG)

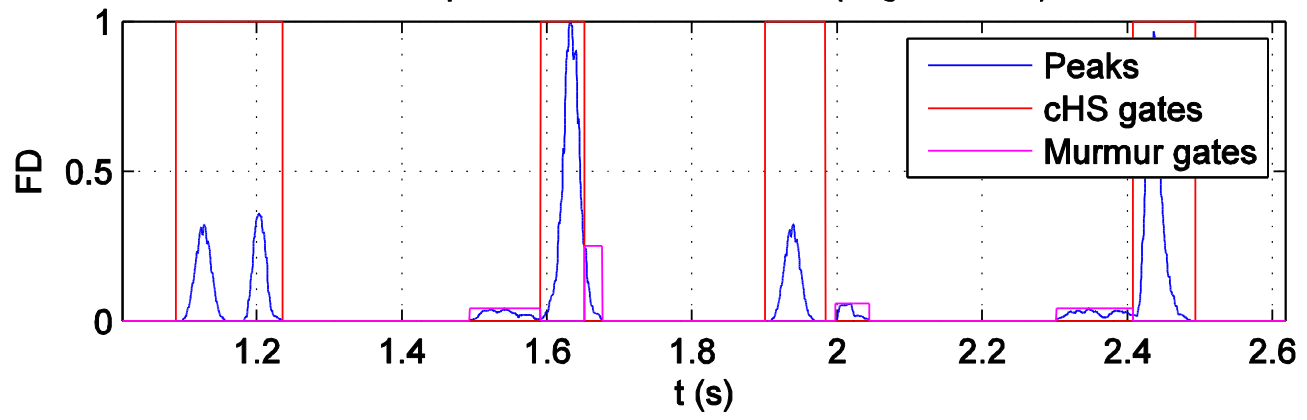

Figure 5-7: The opening snap murmur is misidentified as a split S2 component because the murmur's peak is above the threshold (subplot-2). Also, the right boundary of S1 is repositioned despite the lack of a systolic murmur, and the remaining piece is misidentified as a murmur [dwt_michigan.m]. 
Figure 5-8 illustrates an error that is unrelated to either energy thresholding or the trim_HS() function. Here, find_heart_cycles() determines an incorrect distance between cycle boundaries (subplot-1). This is because the PCG has a large amount of activity, so the autocorrelation waveform is jagged, and a peak is detected near zero lag. This peak's magnitude is greater than the first spike located at a lag of one second, so it is misidentified as the first heart cycle boundary. Segmentation therefore fails because the cycle durations are too short.
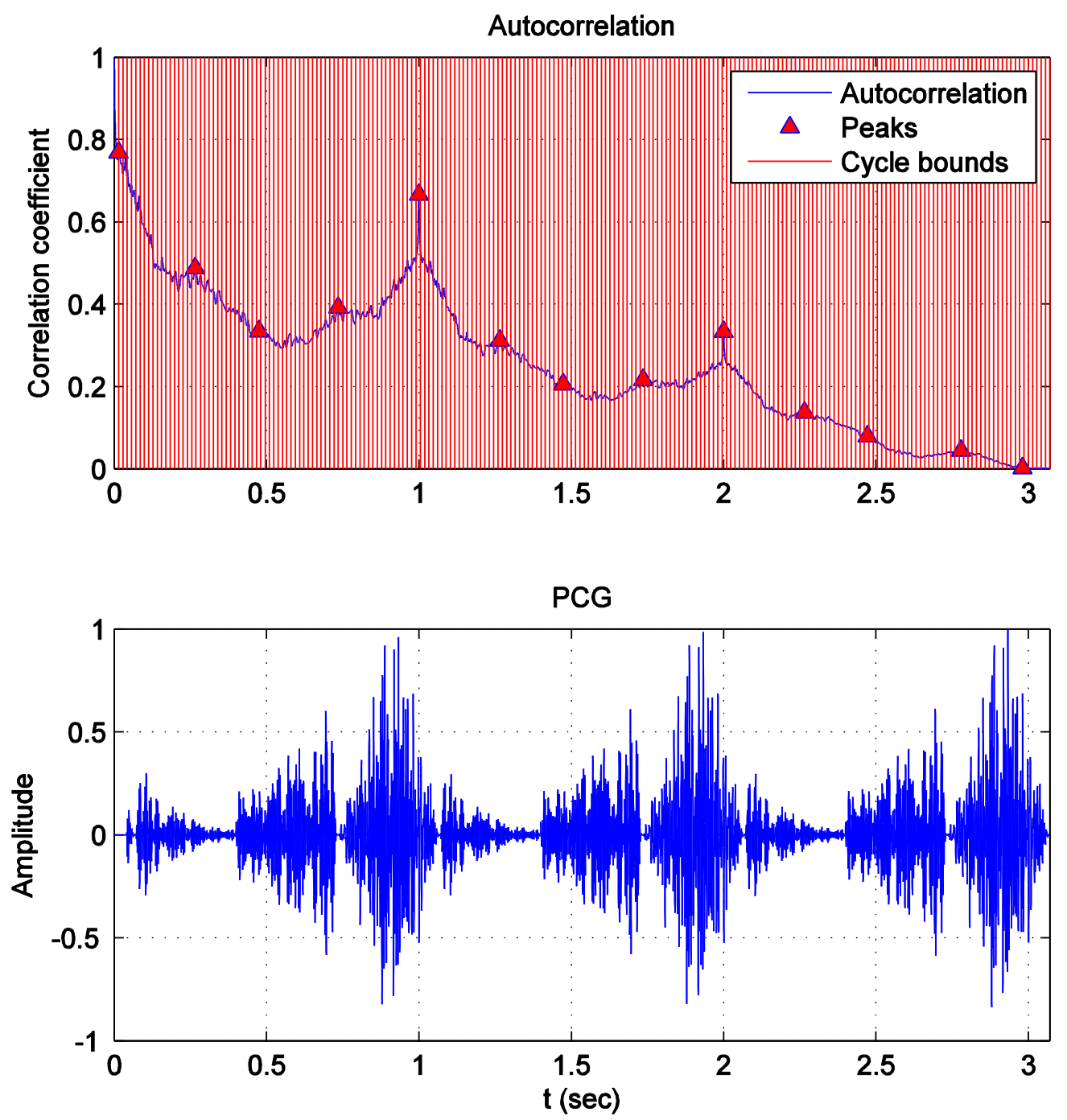

Figure 5-8: The cycle durations are too short because a peak near zero lag is misidentified as a heart cycle boundary [“AP.mp3", dwt_littmann.m]. 


\subsubsection{Wavelet Results}

The wavelet-based segmentation errors described in the previous section are labeled and summarized in Table 5-9. All of the murmur-containing sound files, as well as the murmur-free sound files with detected errors, are listed in Table 5-10 (Michigan) and Table 5-11 (Littmann). For each sound file, the number of actual murmurs, detected true murmurs, and heart cycles with detected false murmurs are listed; and the sound files are labeled with their detected errors. This data is used to determine the TMDR in Table 5-12 and the FMDR in Table 5-13. Most of the sound files with detected errors either lower the TMDR or increase the FMDR, or both, but certain sound files with detected errors do not actually result in undetected true murmurs or detected false murmurs and therefore do not affect those rates.

Table 5-9: Wavelet-based segmentation error labels and descriptions.

\begin{tabular}{|c|c|c|}
\hline Error & Process & Description \\
\hline E1-A & \multirow{3}{*}{ Energy thresholding } & $\mathrm{S} 1 / \mathrm{S} 2<\mathrm{HS}$ threshold \\
\hline E1-B & & S3/S4 < HS threshold \\
\hline E1-C & & Murmur > HS threshold \\
\hline$E 2-A$ & \multirow{3}{*}{ trim_HS() } & HS peak < segment threshold \\
\hline E2-B & & Murmur peak > segment threshold \\
\hline E2-C & & $\begin{array}{l}\text { Trough is identified as a junction } \\
\text { between HS and murmur }\end{array}$ \\
\hline E3 & find_heart_cycles() & $\begin{array}{l}\text { Incorrect peak detected in the } \\
\text { autocorrelation signal }\end{array}$ \\
\hline
\end{tabular}


Table 5-10: Wavelet-based segmentation results (Michigan).

\begin{tabular}{|c|c|c|c|c|}
\hline Filename & Error & $\begin{array}{c}\text { Total } \\
\text { Murmurs }\end{array}$ & $\begin{array}{l}\text { Detected true } \\
\text { murmurs }\end{array}$ & $\begin{array}{c}\text { Heart cycles with } \\
\text { detected false } \\
\text { murmurs }\end{array}$ \\
\hline early dias murm & $E 1-A$ & 5 & 0 & 3 \\
\hline $\begin{array}{l}\text { ejection click \& syst } \\
\text { eject murm \& single s2 }\end{array}$ & & 4 & 4 & 0 \\
\hline mid sys click & & 5 & 5 & 0 \\
\hline OS \& dias murm & $\begin{array}{l}\text { E2-B } \\
\text { E2-C }\end{array}$ & 5 & 5 & 1 \\
\hline S3 \& holosys murm & E1-A & 6 & 5 & 2 \\
\hline S4 \& mid sys murm & & 5 & 5 & 0 \\
\hline $\begin{array}{l}\text { sys click \& late sys } \\
\text { murm }\end{array}$ & & 5 & 5 & 0 \\
\hline sys murm \& absent S2 & & 6 & 6 & 0 \\
\hline sys \& dias murm & & 6 & 6 & 0 \\
\hline $\begin{array}{l}\text { sys eject murm \& split } \\
\text { S2 trans }\end{array}$ & E1-B & 6 & 6 & 1 \\
\hline $\begin{array}{l}\text { sys eject murm \& split } \\
\text { S2 pers }\end{array}$ & & 5 & 5 & 0 \\
\hline normal 2 & E1-A & 0 & 0 & 4 \\
\hline S3 & E2-A & 0 & 0 & 5 \\
\hline S4 & E2-A & 0 & 0 & 5 \\
\hline \multicolumn{2}{|l|}{ Total } & 58 & 52 & 21 \\
\hline
\end{tabular}


Table 5-11: Wavelet-based segmentation results (Littmann).

\begin{tabular}{|c|c|c|c|c|}
\hline Filename & Errors & $\begin{array}{c}\text { Total } \\
\text { Murmurs }\end{array}$ & $\begin{array}{l}\text { Detected true } \\
\text { murmurs }\end{array}$ & $\begin{array}{l}\text { Heart cycles } \\
\text { with detected } \\
\text { false murmurs }\end{array}$ \\
\hline$A P$ & E3 & 4 & 0 & 0 \\
\hline$A R$ & & 4 & 4 & 0 \\
\hline$A S$ & & 3 & 3 & 0 \\
\hline$A S D$ & E1-C & 4 & 2 & 0 \\
\hline COA & & 4 & 4 & 0 \\
\hline$E A$ & & 6 & 6 & 0 \\
\hline eject click & & 2 & 2 & 0 \\
\hline $\begin{array}{l}\text { eject click \& AS } \\
\text { moderate \& } A R \text { mild }\end{array}$ & E2-B & 4 & 4 & 0 \\
\hline innocent murmur & & 2 & 2 & 0 \\
\hline late sys click & & 4 & 4 & 0 \\
\hline mid sys click & & 3 & 3 & 0 \\
\hline MR severe & E1-C & 4 & 0 & 0 \\
\hline MS moderate & E1-C & 3 & 2 & 0 \\
\hline MVP & & 4 & 4 & 0 \\
\hline OS & & 2 & 2 & 0 \\
\hline$P D A$ & E1-A & 4 & 2 & 0 \\
\hline S4 \& AS severe & & 2 & 2 & 0 \\
\hline TR severe & & 4 & 4 & 0 \\
\hline$V S D$ & $\mathrm{E} 1-\mathrm{C}$ & 4 & 2 & 0 \\
\hline S4 & $\mathrm{E} 1-\mathrm{B}$ & 0 & 0 & 0 \\
\hline sum gallop & E1-B & 0 & 0 & 2 \\
\hline \multicolumn{2}{|l|}{ Total } & 67 & 52 & 2 \\
\hline
\end{tabular}

Table 5-12: Wavelet-based segmentation true murmur detection rate (TMDR).

\begin{tabular}{|l|c|c|c|}
\hline Dataset & Murmurs & Detected true murmurs & TMDR \\
\hline Michigan & 58 & 52 & $90 \%$ \\
\hline Littmann & 67 & 52 & $78 \%$ \\
\hline Total & 125 & 104 & $83 \%$ \\
\hline
\end{tabular}

Table 5-13: Wavelet-based segmentation false murmur detection rate (FMDR).

\begin{tabular}{|l|c|c|c|}
\hline Dataset & $\begin{array}{c}\text { Heart } \\
\text { Cycles }\end{array}$ & $\begin{array}{c}\text { Heart cycles with } \\
\text { detected false murmurs }\end{array}$ & FMDR \\
\hline Michigan & 101 & 21 & $21 \%$ \\
\hline Littmann & 76 & 2 & $3 \%$ \\
\hline Total & 177 & 23 & $13 \%$ \\
\hline
\end{tabular}




\subsection{Simplicity-Based Segmentation}

\subsubsection{Simplicity Constants}

The constants for simplicity-based segmentation, which consist of window lengths, the peak peeling stopping condition, thresholds, and simplicity-specific values, are listed in Table 5-14. All constants can be changed from their defaults using name-value pair arguments passed to simpl_segment().

Table 5-14: Simplicity-based segmentation constants.

\begin{tabular}{|l|l|l|}
\hline Constant & Value & Description \\
\hline STC & $20 \mathrm{~ms}$ & $\begin{array}{l}\text { Fractal dimension and energy waveform window } \\
\text { lengths }\end{array}$ \\
\hline$N$ & $10^{-4}$ & Peak peeling stopping condition \\
\hline$m$ & $10 \mathrm{~ms}$ & Simplicity window length \\
\hline gamma & $2 \mathrm{~ms}$ & Simplicity delay vector length \\
\hline HS_thresh & 0.8 & Coarseness of the PWC simplicity waveform \\
\hline extra_HS_thresh & 0.6 & Simplicity heart sound threshold \\
\hline WS & $20 \mathrm{~ms}$ & Simplicity extra heart sound threshold \\
\hline min_pk & 0.2 & $\begin{array}{l}\text { PCG smoothing window length } \\
\text { minimum peak height for a split sound component }\end{array}$ \\
\hline
\end{tabular}

\subsubsection{Simplicity Errors}

Simplicity thresholding for simplicity-based segmentation is the functional equivalent of energy thresholding for wavelet-based segmentation, except that the heart sound and murmur segments are identified by their levels simplicity rather than their maximum energies. As such, heart sound segments are misidentified as murmurs when their levels 
are less than the heart sound threshold, and murmur segments are misidentified as heart sounds when their levels are greater than the heart sound threshold.

Figure 5-9 illustrates how murmur segments can be misidentified as heart sounds when their simplicity levels are greater than the normal heart sound threshold. This is the same example used in Figure 5-5 for wavelet-based segmentation, so like before, the two high-frequency systolic murmurs resemble typical murmurs, whereas the diastolic murmur has low frequency content and resembles a heart sound (subplot-1). As a result, both of the systolic murmurs are properly identified because their levels are less than the threshold, but the diastolic murmur pieces are misidentified as heart sounds because their levels are greater than the threshold (subplot-2). 


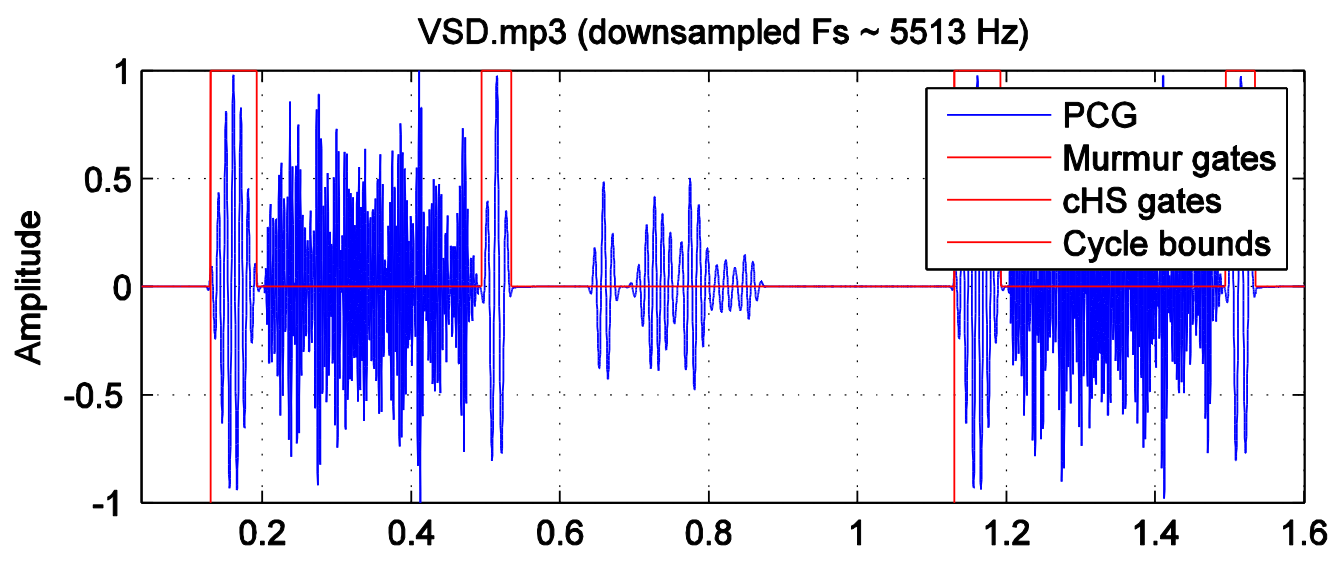

Threshold segments
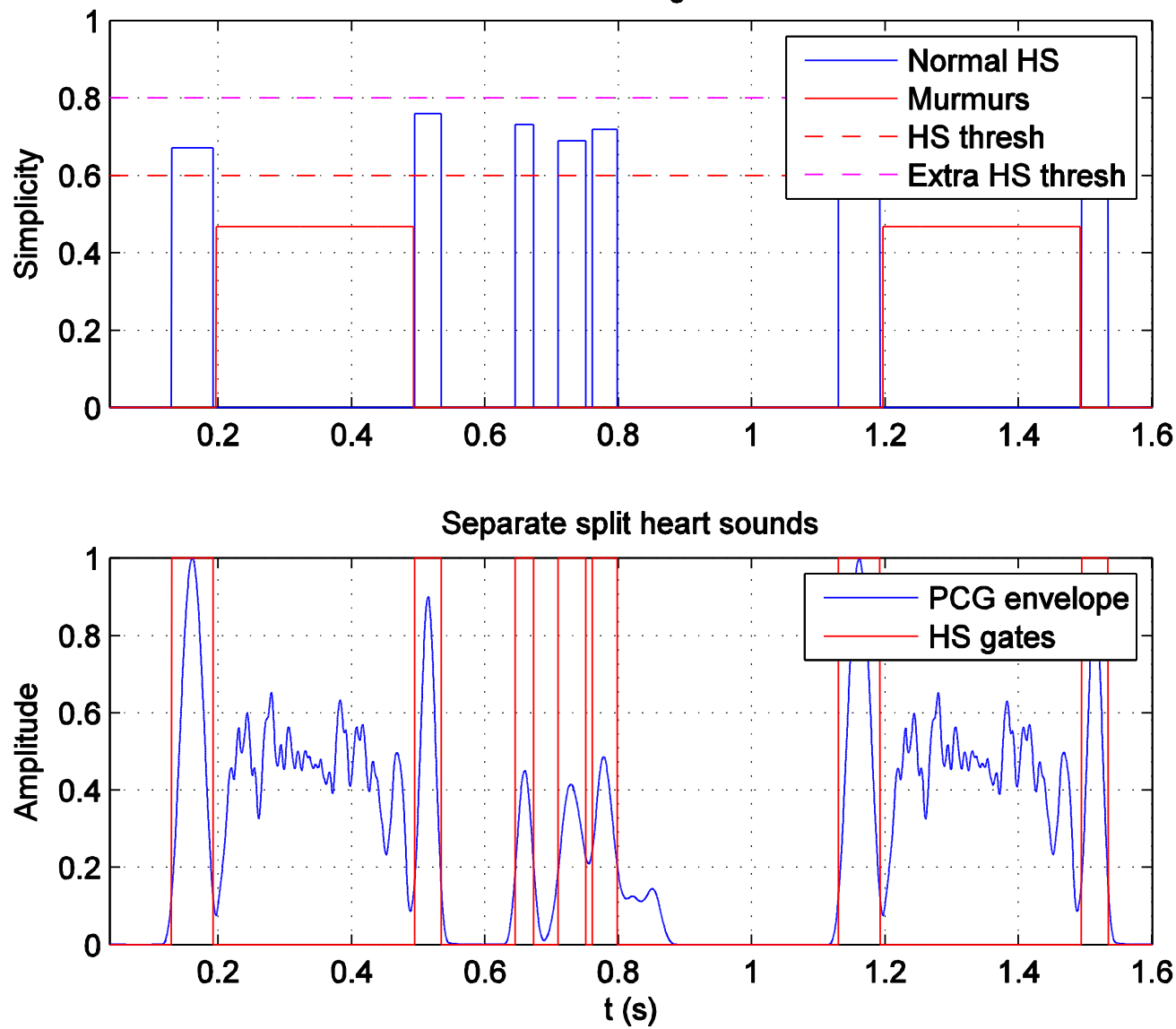

Figure 5-9: The diastolic murmur is misidentified as a heart sound because its simplicity levels are greater than the HS threshold (subplot-2) [simpl_littmann.m]. 
Figure 5-10 illustrates how at least one heart sound segment is required for segmentation. Since all the segments' levels are less than the normal heart sound threshold, no heart sounds are detected (subplot-5). This is the same example used in Figure 5-8 for wavelet-based segmentation, except that the error here occurs prior to find_heart_cycles(), as heart cycle segmentation requires at least one heart sound segment. Therefore, even though the segment levels are less than the threshold, the heart sounds are not misidentified as murmurs because segmentation fails before sound classification can occur. 

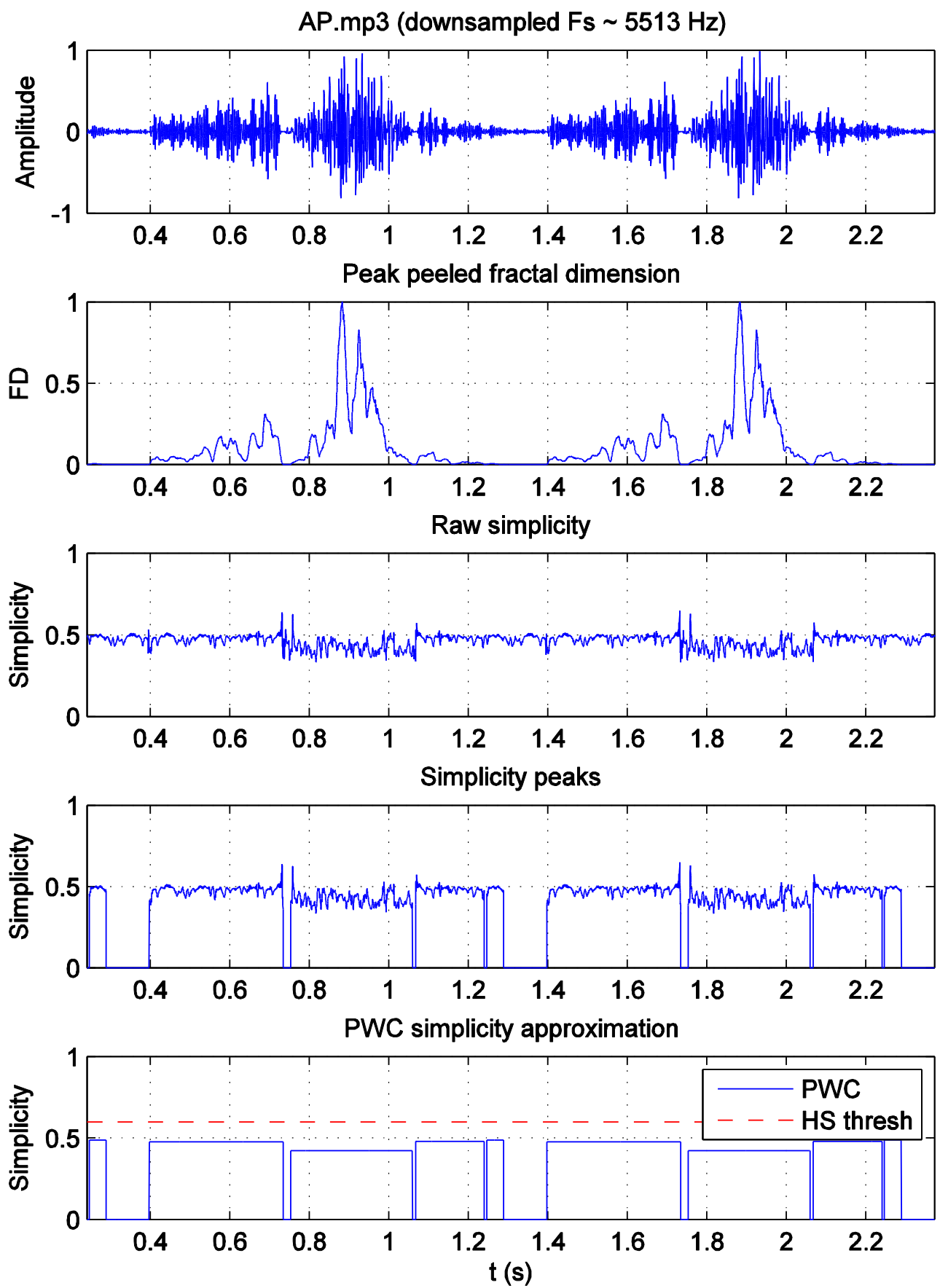

Figure 5-10: No heart sounds are detected because all segment levels are less than the HS threshold (subplot-5) [simpl_littmann.m]. 
The purpose of peak peeling for segmentation is to zero the low-amplitude noise within the silent segments, so that the heart sounds and murmurs can be segmented. Unfortunately, low amplitude murmurs may be inadvertently zeroed as well. In subplot-1 of Figure 5-11, a very low intensity diastolic murmur is not visible on the plot but can be heard as a soft "wooshing" sound with headphones. Further proof of the murmur's existence can be seen in the simplicity waveform in subplot-3. The high simplicity values in systole are typical of the attenuated and smoothed noise in the silent segments, but the moderate simplicity values in diastole, which are lower than those in S1 and S2, are characteristic of murmurs. Since the murmurs here are so soft, their time-domain values are not transformed into fractal values of sufficient amplitude, so they are zeroed during peak peeling (subplot-2). The zero-valued samples in the peak peeled fractal dimension are then used to zero the corresponding samples in the simplicity waveform (subplot-4). As a result, the murmurs cannot be segmented because their simplicity levels are set to zero. This is a rare error because most heart sounds are loud enough to be transformed into a suitable fractal value (and to also be visible on the plot) and are therefore not removed during peak peeling. Nonetheless, this example demonstrates how the amplitude-invariant simplicity transform can be limited by the fractal dimension. 

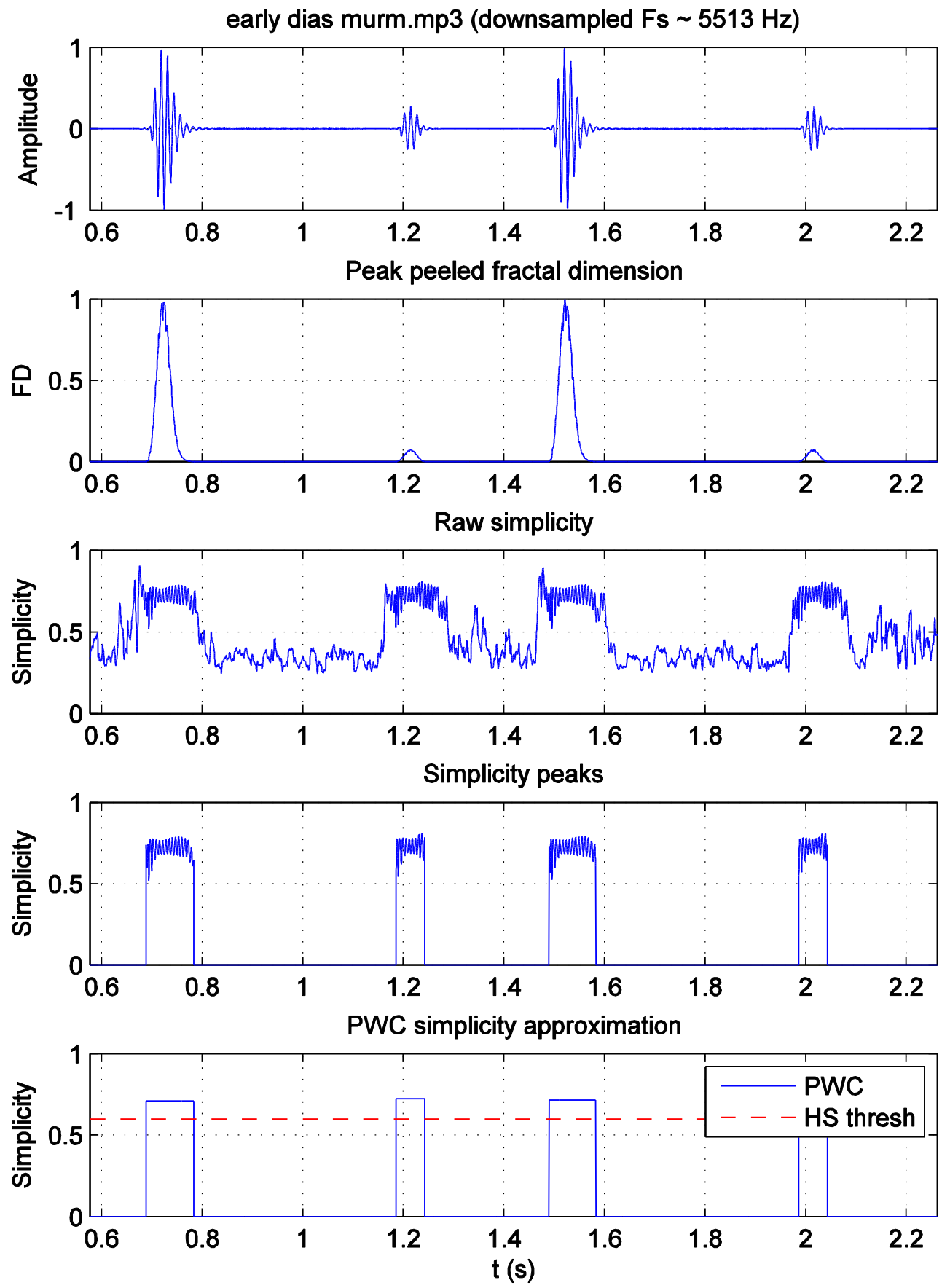

Figure 5-11: The low amplitude diastolic murmurs (not visible) are undetected because they were zeroed while peak peeling the fractal dimension (subplot-2). The corresponding simplicity values are zeroed (subplot-4), so the murmurs are not segmented (subplot5) [simpl_michigan.m]. 
Even though distinguishing extra heart sounds from split sound components is not a requirement for successful segmentation, misidentifying extra heart sounds can affect the segmentation of normal heart sounds in certain scenarios. This is illustrated in Figure 5-12, where the simplicity levels of the summation gallops are greater than the S1 and S2 levels but are nonetheless less than the extra heart sound threshold. As a result, the summation gallops are misidentified as normal heart sounds (subplot-2), and since S2 and the summation gallop are separated by less than sscope.min_syst_dur samples, they are further misidentified as split sound components. Unfortunately, the distance separating the "split" sounds from S1 is less than the distance separating $S 1$ from $S 2$, so systole and diastole, and therefore S1 and S2, are switched. 


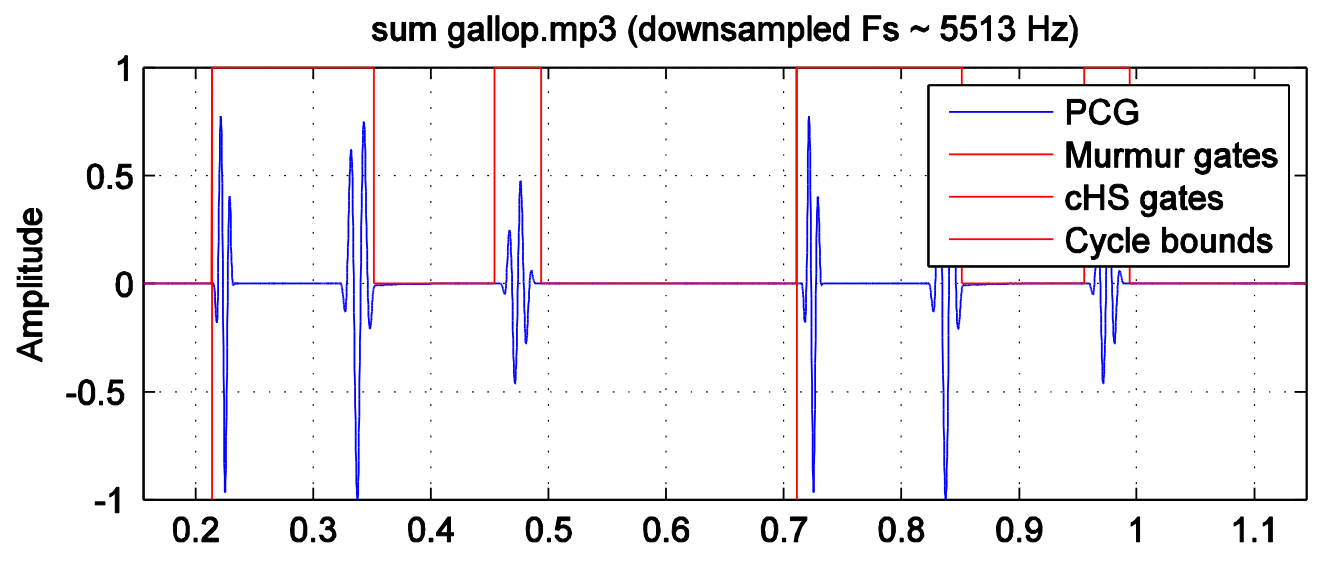

Threshold segments
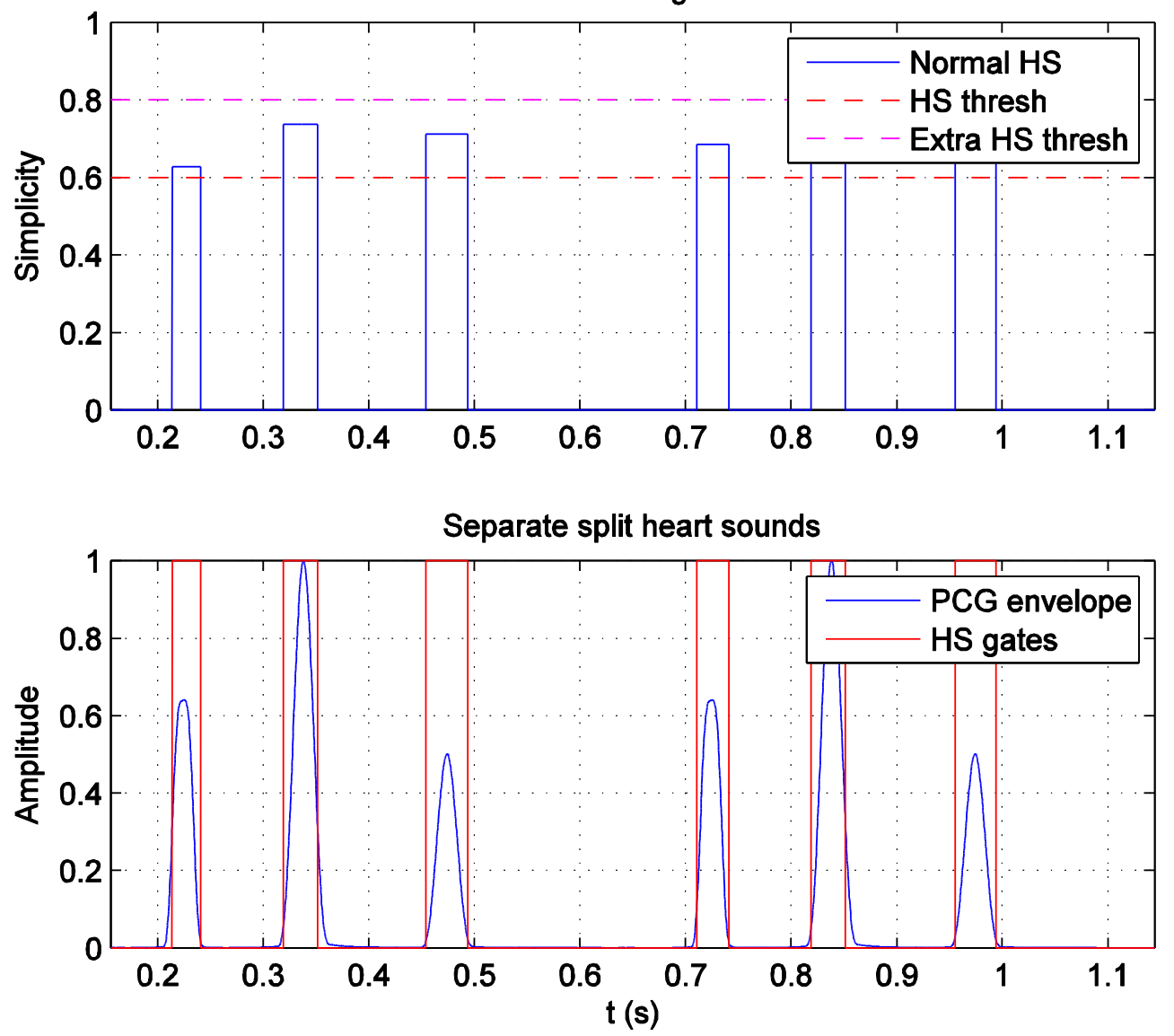

Figure 5-12: The summation gallops are misidentified as split sound components because their simplicity levels are less than the extra HS threshold (subplot-2). This causes systole and diastole, and therefore S1 and S2, to be switched (subplot-1) [simpl_littmann.m]. 


\subsubsection{Simplicity Error Tables}

The simplicity-based segmentation errors described in the previous section are labeled and summarized in Table 5-15. All the murmur-containing sound files, as well as the murmur-free sound files with detected errors, are listed in Table 5-16 (Michigan) and Table 5-17 (Littmann). For each sound file, the number of actual murmurs, detected true murmurs, and cycles with detected false murmurs are listed; and the sound files are labeled with their detected errors. This data is used to determine the TMDR in Table 5-18 and the FMDR in Table 5-19. Most sound files with detected errors either lower the TMDR or increase the FMDR, or both, but certain sound files with detected errors do not actually cause undetected true murmurs or detected false murmurs and therefore do not affect those rates at all.

Table 5-15: Simplicity-based segmentation error labels and descriptions.

\begin{tabular}{|l|c|l|}
\hline Error & Process & Description \\
\hline E1-A & & $\mathrm{S} 1 / \mathrm{S} 2<$ normal HS threshold \\
\cline { 1 - 1 } E1-B & Simplicity thresholding & Murmur $>$ normal HS threshold \\
E1-C & & S3/S4 $<$ extra HS threshold \\
\hline E2 & Peak peeling & Soft murmurs are removed \\
\hline
\end{tabular}


Table 5-16: Simplicity-based segmentation results (Michigan).

\begin{tabular}{|l|c|c|c|c|}
\hline Filename & Errors & Murmurs & $\begin{array}{c}\text { Detected true } \\
\text { murmurs }\end{array}$ & $\begin{array}{c}\text { Cycles with } \\
\text { detected false } \\
\text { murmurs }\end{array}$ \\
\hline early dias murm & E2 & 5 & 0 & 0 \\
\hline $\begin{array}{l}\text { ejection click \& syst } \\
\text { eject murm \& single } \\
\text { S2 }\end{array}$ & & 4 & 4 & 0 \\
\hline mid sys click & E1-B & 5 & 5 & 0 \\
\hline OS \& dias murm & 6 & 4 & 0 \\
\hline $\begin{array}{l}\text { S3 \& holosys murm } \\
\text { S4 \& mid sys murm }\end{array}$ & 5 & 5 & 0 \\
\hline $\begin{array}{l}\text { sys click \& late sys } \\
\text { murm }\end{array}$ & 5 & 5 & 0 \\
\hline $\begin{array}{l}\text { sys murm \& absent } \\
\text { S2 }\end{array}$ & 6 & 6 & 6 & 0 \\
\hline sys \& dias murm & 6 & 6 & 6 & 0 \\
\hline $\begin{array}{l}\text { sys eject murm \& } \\
\text { split S2 trans }\end{array}$ & & 58 & 52 & 0 \\
\hline $\begin{array}{l}\text { sys eject murm \& } \\
\text { split S2 pers }\end{array}$ & & & & 0 \\
\hline \multicolumn{1}{|c|}{ Total } & & 5 & 6 & 0 \\
\hline
\end{tabular}


Table 5-17: Simplicity-based segmentation results (Littmann).

\begin{tabular}{|c|c|c|c|c|}
\hline Filename & Errors & Murmurs & $\begin{array}{l}\text { Detected } \\
\text { true } \\
\text { murmurs }\end{array}$ & $\begin{array}{l}\text { Cycles with } \\
\text { detected false } \\
\text { murmurs }\end{array}$ \\
\hline$A P$ & E1-A & 4 & 0 & 0 \\
\hline$A R$ & & 4 & 4 & 0 \\
\hline$A S$ & & 3 & 3 & 0 \\
\hline$A S D$ & E1-B & 4 & 2 & 0 \\
\hline COA & & 4 & 4 & 0 \\
\hline$E A$ & & 6 & 6 & 0 \\
\hline eject click & & 2 & 2 & 0 \\
\hline $\begin{array}{l}\text { eject click \& AS } \\
\text { moderate \& } A R \text { mild }\end{array}$ & & 4 & 4 & 0 \\
\hline innocent murmur & & 2 & 2 & 0 \\
\hline late sys click & & 4 & 4 & 0 \\
\hline mid sys click & & 3 & 3 & 0 \\
\hline MR severe & E1-B & 4 & 2 & 0 \\
\hline MS moderate & E1-B & 3 & 0 & 0 \\
\hline MVP & & 4 & 4 & 0 \\
\hline OS & & 2 & 2 & 0 \\
\hline$P D A$ & E1-A & 4 & 2 & 0 \\
\hline S4 \& AS severe & & 2 & 2 & 0 \\
\hline TR severe & & 4 & 4 & 0 \\
\hline$V S D$ & E1-B & 4 & 2 & 0 \\
\hline sum gallop & E1-C & 0 & 0 & 0 \\
\hline \multicolumn{2}{|l|}{ Total } & 67 & 52 & 0 \\
\hline
\end{tabular}

Table 5-18: Simplicity true murmur detection rate (TMDR).

\begin{tabular}{|l|c|c|c|}
\hline Dataset & Murmurs & $\begin{array}{c}\text { Detected true } \\
\text { murmurs }\end{array}$ & TMDR \\
\hline Michigan & 58 & 52 & $90 \%$ \\
\hline Littmann & 67 & 52 & $78 \%$ \\
\hline Total & 125 & 104 & $83 \%$ \\
\hline
\end{tabular}

Table 5-19: Simplicity false murmur detection rate (FMDR).

\begin{tabular}{|l|c|c|c|}
\hline Dataset & Cycles & $\begin{array}{c}\text { Cycles with detected } \\
\text { false murmurs }\end{array}$ & FMDR \\
\hline Michigan & 101 & 0 & $0 \%$ \\
\hline Littmann & 76 & 0 & $0 \%$ \\
\hline Total & 177 & 0 & $0 \%$ \\
\hline
\end{tabular}




\subsection{Comparison of Segmentation Error Performance for the Two Methods}

The various detection rates for wavelet and simplicity-based segmentation are compared in Table 5-20 and discussed below. In addition, the errors types described in Table 5-9 (wavelet-based segmentation) and Table 5-15 (simplicity-based segmentation) are compared among sounds files in Table 5-21 (Michigan datest) and Table 5-22 (Littmann dataset).

Table 5-20: Wavelet and simplicity-based segmentation performance comparison.

\begin{tabular}{|l|c|c|c|c|}
\hline Method & FNDR & FPDR & TMDR & FMDR \\
\hline Wavelet & $4 \%$ & $22 \%$ & $83 \%$ & $13 \%$ \\
\hline Simplicity & $9 \%$ & $0 \%$ & $83 \%$ & $0 \%$ \\
\hline
\end{tabular}

The TMDR is the same for both wavelet and simplicity-based segmentation because the incidence of detecting true murmurs is lowered by the detection of false heart sounds (murmurs misclassified as heart sounds). For wavelet-based segmentation, murmurs are typically loud enough to be greater than the energy threshold and are misclassified as heart sounds when the wavelet filter is incapable of attenuating the murmurs. For simplicity-based segmentation, the same murmurs that cannot be attenuated by the wavelet filter tend to also have high simplicity and are likewise misclassified as murmurs. This common susceptibility for errors is due to the simplicity and wavelet transforms' dependence on waveform morphology. In fact, most of the sound files with detected false heart sounds are common to both wavelet-based segmentation (E1-C) and simplicitybased segmentation (E1-B).

The FNDR is low for both segmentation methods due to its inverse relationship with the TMDR. However, the primary reason the FNDR for wavelet-based segmentation is less than the FNDR for simplicity-based segmentation is that the FMDR is non-zero for wavelet-based segmentation. This is because detecting false murmurs in murmurcontaining cycles that lack detected true murmurs will artificially decrease the number of 
false negative cycles. This is aptly demonstrated in the sound file early dias murm from the Michigan dataset. For simplicity-based segmentation, all five cycles are false negatives because the soft murmurs were zeroed through peak peeling (E2). For waveletbased segmentation, the same murmurs were also zeroed through peak peeling; however, only two of the cycles are false negatives because false murmurs were detected in the other three cycles. Therefore, the FNDR is suitable for clinical evaluations, but a full performance evaluation of the two methods can only be achieved by comparing the TMDR and FMDR. As a result, simplicity-based segmentation, despite having the same TMDR as wavelet-based segmentation, is superior in this regard because its FMDR is zero.

The FMDR is non-zero for wavelet-based segmentation because heart sounds are often soft enough to be less than the heart sound energy threshold and therefore misclassified as murmurs (E1-A and E1-B). Furthermore, trim_HS() is prone to splitting heart sound segments and creating unnecessary murmurs (E2-A and E2-C). In contrast, the FMDR is zero for simplicity-based segmentation because the simplicity waveform is amplitude invariant. Since heart sounds have relatively high simplicity, it is rare for them to be misclassified as murmurs or noise (low simplicity). In addition, the gamma constant for the L2 Potts minimization function is the equivalent of trim_HS() for simplicity-based segmentation because both are used to determine the optimal boundaries between merged heart sounds and murmurs. Nonetheless, the two are hardly equivalent in outcome because locating the segment boundaries using the piecewise constant approximation function on the simplicity waveform is a far more accurate and less error prone method than is using energy thresholding and trim_HS().

The FPDR, as a subset of the FMDR, is likewise non-zero for wavelet-based segmentation but zero for simplicity-based segmentation. The only reason the FPDR is 
greater than the FMDR, at least for these datasets, is that a higher proportion of false murmurs are detected in the murmur-free cycles than are detected in all cycles.

Table 5-21: Wavelet and simplicity-based segmentation error comparisons (Michigan).

\begin{tabular}{|l|c|c|}
\hline Filename & Wavelet & Simplicity \\
\hline early dias murm & E1-A & E2 \\
\hline $\begin{array}{l}\text { ejection click \& syst eject } \\
\text { murm \& single S2 }\end{array}$ & & \\
\hline mid sys click & & E1-B \\
\hline OS \& dias murm & E2-B & \\
\hline S3 \& holosys murm & E2-C & \\
\hline S4 \& mid sys murm & & E2 \\
\hline sys click \& late sys murm & & \\
\hline sys murm \& absent S2 & & \\
\hline sys \& dias murm & & \\
\hline sys eject murm \& split & E1-B & \\
S2 trans & & \\
\hline sys eject murm \& split & & \\
S2 pers & & \\
\hline normal 1 & E1-A & \\
\hline normal 2 & E2-A & \\
\hline S3 & & \\
\hline S4 & & \\
\hline single S2 & & \\
\hline split S1 pers & & \\
\hline split S2 pers & & \\
\hline split S2 trans & & \\
\hline
\end{tabular}


Table 5-22: Wavelet and simplicity-based segmentation error comparisons (Littmann)

\begin{tabular}{|l|c|c|}
\hline File Name & Wavelet & Simplicity \\
\hline$A P$ & E3 & E1-A \\
\hline$A R$ & & E1-B \\
\hline$A S$ & E1-C & \\
\hline$A S D$ & & \\
\hline COA & & \\
\hline EA & & \\
\hline eject click & E2-B & \\
\hline $\begin{array}{l}\text { eject click \& AS } \\
\text { moderate \& } \text { AR mild }\end{array}$ & & E1-B \\
\hline innocent murmur & & \\
\hline late sys click & & \\
\hline mid sys click & E1-C \\
\hline MR severe & E1-C & \\
\hline MS moderate & & E1-A \\
\hline MVP & & \\
\hline OS & E1-A & \\
\hline PDA & & E1-B \\
\hline S4 \& AS severe & & \\
\hline TR severe & & \\
\hline VSD & & \\
\hline normal & & \\
\hline S3 \& S4 & & \\
\hline S3 abnormal & & \\
\hline S3 physio & & \\
\hline S4 & & \\
\hline sum gallop & & \\
\hline split S2 fixed & & \\
\hline split S2 physio & & \\
\hline split S1 & & \\
\hline
\end{tabular}




\section{Conclusions and Future Work}

The results from Chapter 5 demonstrate how simplicity-based segmentation is superior to wavelet-based segmentation. This is because the simplicity transform is amplitude invariant and, in combination with Pottslab's piecewise constant denoising algorithm, is able to determine each sound segment's optimal boundaries, assign a single simplicity value to each segment, and then classify the sound segments according to their simplicity levels. This is in contrast to wavelet-based segmentation, which must attenuate the murmurs to segment the heart sounds, remove non-attenuated or partially attenuated murmur segments by energy thresholding, and use the PCG waveform to remove any murmurs merged with heart sounds. As a result, the incidence of false murmurs, and therefore false positives, is significant. Despite the comparable false negative and true murmur detection rates for both methods, simplicity-based segmentation is ultimately preferable due to its zero false positive and false murmur detection rates.

Even though both segmentation methods are adequate for detecting and classifying murmurs, certain aspects of the system could be improved. One improvement could be applied to the heart cycle segmentation function, find_heart_cycles(). This function determines the heart cycle boundaries by locating the largest peak in the autocorrelation waveform, which should resemble a spike and be located on the first heart cycle's stop boundary, and then places the remaining heart cycle boundaries at integer multiples of the first peak's location. Unfortunately, this function is currently incapable of verifying whether or not the largest peak is a prominent spike rather than a small fluctuation. This could be improved by setting a minimum acceptable peak prominence, or height relative to surrounding troughs, by using the 'MinPeakProminence' option for findpeaks(), which is only available in MATLAB 2015. 
Another suggested improvement would replace the simple thresholds used during simplicity-based segmentation with a clustering algorithm. Rather than manually choosing an optimal threshold, sound segment features such as pitch, location, shape, and duration could be placed in a feature vector, and the algorithm would use these features to classify each sound segment as either a normal heart sound, extra heart sound, or murmur. Finally, even though the purpose of this system is to detect and classify murmurs as either systolic or diastolic, it is also desirable to further categorize the murmurs by their specific types, such as aortic stenosis, mitral regurgitation, atrial septal defect, etc. This could be achieved by reusing the feature vector from the clustering operation as an input to an artificial neural network. This would require training the network with a select dataset of PCG's, so that murmur segments from clinical PCG's could be completely and accurately classified. 


\section{REFERENCES}

[1] J. Doohan, "The Circulatory System," Santa Barbara City College, 17 September 1999. [Online]. Available: http://www.biosbcc.net/doohan/sample/htm/heart.htm. [Accessed 18 February 2016].

[2] Wapcaplet, Diagram of the Human Heart (Cropped), Wikimedia Commons.

[3] R. Klabunde, "Cardiac Cycle," 2 January 2011. [Online]. Available: http://www.cvphysiology.com/Heart\%20Disease/HD002.htm. [Accessed 19 February 2016].

[4] M. R. Villarreal, Human Heart during Systole and Diastole, Wikimedia Commons, 2008.

[5] P. Kubin, "The Stethoscope and How to Use It," 4 November 2012. [Online]. Available: http://www.mypatraining.com/stethoscope-and-how-to-use-it. [Accessed 20 April 2015].

[6] S. Lome, "Heart Murmurs," Healio, [Online]. Available: http://www.healio.com/cardiology/learn-the-heart/cardiology-review/heart-murmurs. [Accessed 20 April 2015].

[7] "Phonocardiography," Encyclopaedia Britannica., 2016. [Online]. Available: http://www.britannica.com/science/phonocardiography. [Accessed 27 February 2016].

[8] S. Lome, "Heart Sounds," [Online]. Available: http://www.learntheheart.com/cardiology-review/heart-sounds/. [Accessed 20 April 2015]. 
[9] A. Kumar and T. V. Ananthapadmanabha, "Heart Rate Variability using Shannon Energy," SASTech, vol. V, no. 2, pp. 23-26, 2006.

[10] L. J. Hadjileontiadis and I. T. Rekanos, "Detection of Explosive Lung and Bowel Sounds by Means of Fractal Dimension," IEEE Signal Processing Letters, vol. 10, no. 10, pp. 311-314, 2003.

[11] L. J. Hadjileontiadis, "Wavelet-Based Enhancement of Lung and Bowel Sounds Using Fractal Dimension Thresholding-Part I: Methodology," IEEE Transactions on Biomedical Engineering, vol. 52, no. 6, pp. 1143-1148, 2005.

[12] N. A. a. T. Ning, "Isolation of Systolic Heart Murmurs Using Wavelet Transform and Energy Index," in Congress on Image and Signal Processing, 2008.

[13] V. Nigam and R. Priemer, "Accessing heart dynamics to estimate durations of heart sounds," Institute of Physics Publishing, vol. 26, pp. 1005-1018, 2005.

[14] D. Kumar, P. Carvalho, M. Antunes, J. Henriques, M. Maldonado, R. Schmidt and J. Habetha, "Wavelet Transform and Simplicity Based Heart Murmur Segmentation," Computers in Cardiology, vol. 33, pp. 173-176, 2006.

[15] M. A. Little and N. S. Jones, "Generalized Methods and Solvers for Noise Removal from Piecewise Constant Signals. I. Background Theory.," Proceedings of the Royal Society, vol. 471, no. 2177, 8 June 2011.

[16] A. Weinmann, M. Storath and L. Demaret, "The L1-Potts Functional for Robust Jump-Sparse Reconstruction," arXiv:1207.4642, 2012.

[17] M. Storath, A. Weinmann and L. Demaret, "Jump-Sparse and Sparse Recovery Using Potts Functionals," IEEE Transactions on Signal Processing, vol. 62, no. 14, pp. 3654-3666, 2014. 
[18] M. Storath and A. Weinmann, "Fast Partitioning of Vector-Valued Images," SIAM Journal on Imaging Sciences, vol. 7, no. 3, pp. 1826-1852, 2014.

[19] Assessment Technologies Institute, "Cardiac Examination," Assessment Technologies Institute, [Online]. Available:

http://atitesting.com/ati_next_gen/skillsmodules/content/physical-assessmentadult/equipment/cardiac.html. [Accessed 28 February 2016].

[20] S. G. Wong, "Design, Characterization, and Application of a Multiple Input Stethoscope Apparatus," California Polytechnic State University, San Luis Obispo, 2014.

[21] I. McCowan, Microphone Arrays: A Tutorial, 2001.

[22] J. Scampini, "Overview of Ultrasound Imaging Systems and the Electrical Components Required for Main Subfunctions," Maxim Integrated, 10 May 2010. [Online]. Available: https://www.maximintegrated.com/en/appnotes/index.mvp/id/4696. [Accessed 14 Feb 2016].

[23] Andeggs, Artist, 3D spherical coordinates. [Art]. Wikimedia Commons, 2009.

[24] D. Blanford and J. Parr, Introduction to Digital Signal Processing, Upper Saddle River: Pearson, 2013.

[25] A. Ambardar, Analog and Digital Signal Processing, Pacific Grove: Brooks/Cole, 1999.

[26] B. Stern, "The Basic Concepts of Diagnostic Ultrasound1," Yale-New Haven Teachers Institute, [Online]. Available: http://www.yale.edu/ynhti/curriculum/units/1983/7/83.07.05.x.html. [Accessed 157 2016]. 
[27] J. Meiss, "Dynamical Systems," Scholarpedia, 2007. [Online]. Available: http://www.scholarpedia.org/article/Dynamical_system. [Accessed 8 May 2016].

[28] "The Ptolemaic Model," lowa State University, 2001. [Online]. Available: http://www.polaris.iastate.edu/EveningStar/Unit2/unit2_sub1.htm. [Accessed 6 May 2016].

[29] T. D. Sauer, "Attractor Reconstruction," Scholarpedia, 2006. [Online]. Available: http://www.scholarpedia.org/article/Attractor_reconstruction. [Accessed 8 May 2016].

[30] A. Leon-Garcia, Probability, Statistics, and Random Processes for Electrical Engineering, Upper Saddle River: Pearson Prentice Hall, 2008.

[31] J. Rossel, Artist, Functional approximatin of square wave using 5 harmonics. [Art]. Wikimedia Commons, 2010.

[32] A. W. Martin Storath, "Pottslab," [Online]. Available: http://pottslab.de/. [Accessed 15 July 2016].

[33] "Heart and Lung Sounds," 3M, [Online]. Available: http://www.3m.com/healthcare/littmann/mmm-library.html. [Accessed 167 2016].

[34] M. Rajesh S. Mangrulkar and M. Richard D. Judge, "Heart Sound and Murmur Library," University of Michigan Medical School, Ann Arbor. [Online]. 
APPENDICES 


\section{A. Scripts}

\section{A.1 Examples}

\section{A.1.1 chp4_seg.m}

clear; clc; close all

$\mid \mathrm{vl}=2$

$|\mathrm{vl}| 2=5$

$\% \%$ Split S2 (persistent)

close all

stethoscope('michigan', 'split S2 pers.mp3', Ivl1, 'show_filt', true);

$\% \%$ Systolic ejection murmur \& split S2 (persistent)

close all

sscope = stethoscope('michigan', 'sys eject murm \& split S2 pers.mp3', Ivl1);

sscope = dwt_segment(sscope, lvl2, 'show', 'seg');

plot(sscope)

$\% \%$ Systolic murmur \& absent S2

close all

sscope = stethoscope('michigan', 'sys murm \& absent S2.mp3', Ivl1);

dwt_segment(sscope, Ivl2, 'show', \{

'seg'\%, ...

$\% \quad$ 'peak_peel', ...

$\%$ 'find_cyc'

\}$)$

$\% \%$ Split S2 (fixed)

close all

sscope = stethoscope('littmann', 'split S2 fixed.mp3', Ivl1);

dwt_segment(sscope, Ivl2, 'show', 'seg');

$\% \%$ S3 \& holosystolic murmur

close all

sscope = stethoscope('michigan', 'S3 \& holosys murm.mp3', Ivl1);

simpl_segment(sscope, 'show', 'seg');

\section{A.1.2 energy_functions.m}

$\%$ Shannon energy vs squared energy curves

clear; close all; clc

$x=$ linspace $(0,1,101)$

shannon $=x .{ }^{\wedge} 2 .{ }^{*} \log \left(x .{ }^{\wedge} 2\right)$;

squared $=x .^{\wedge} 2$

figure('color','w')

hold on

plot(x, squared)

plot(x, -shannon, 'm')

grid on 


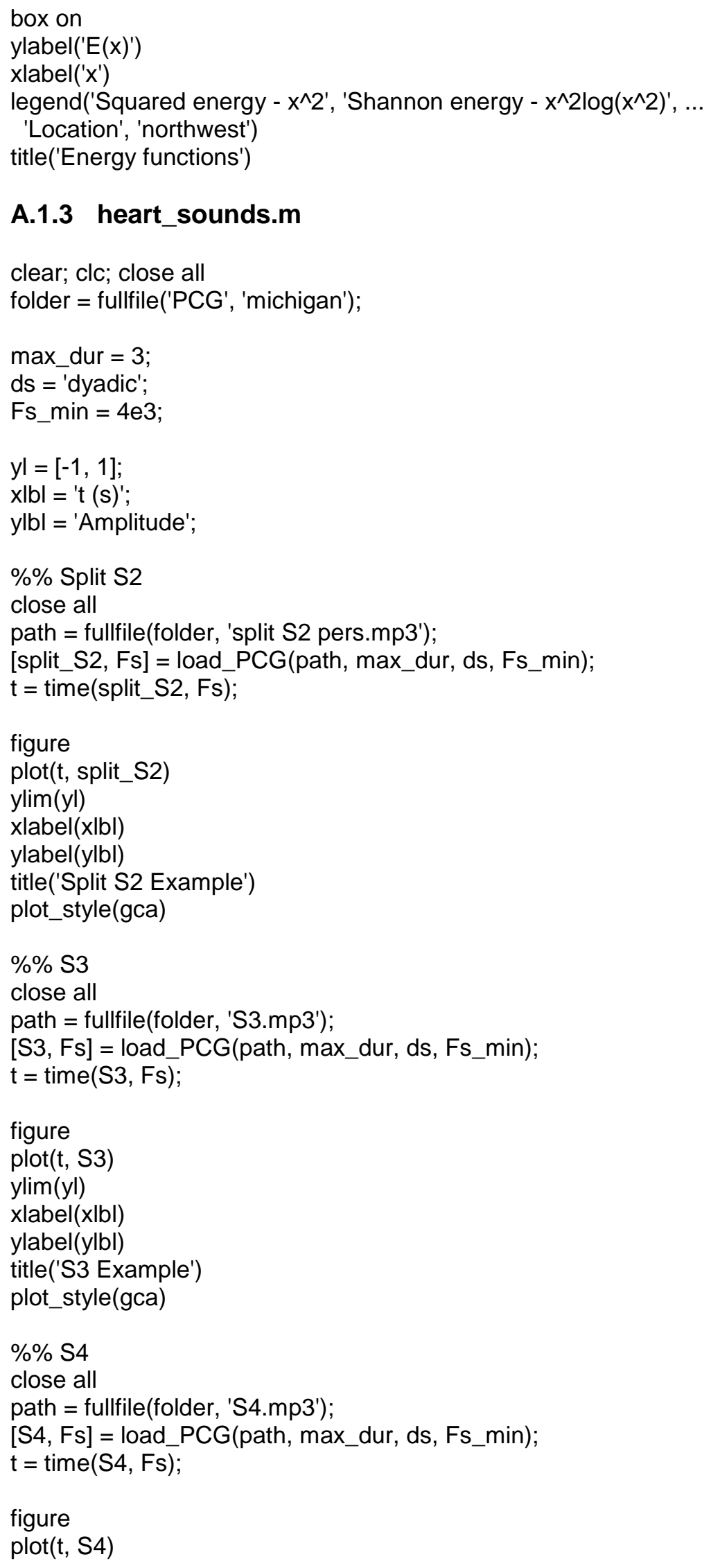

\section{A.1.3 heart_sounds.m}




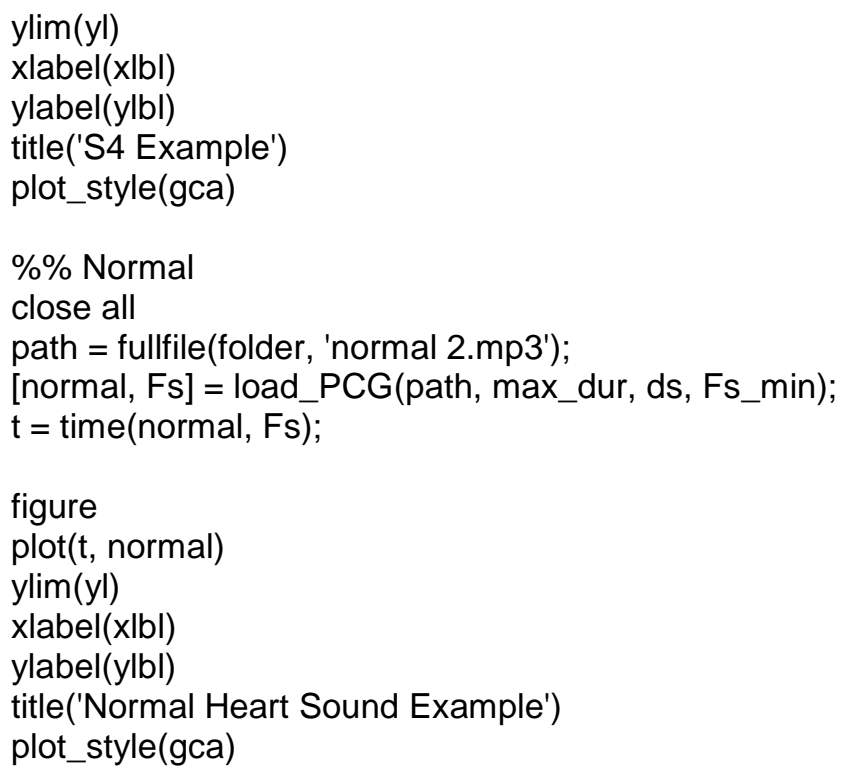

\section{A.1.4 PCG_FFT.m}

$\%$ Compare the spectra of S1, S2, and murmur.

clc; close all; clear

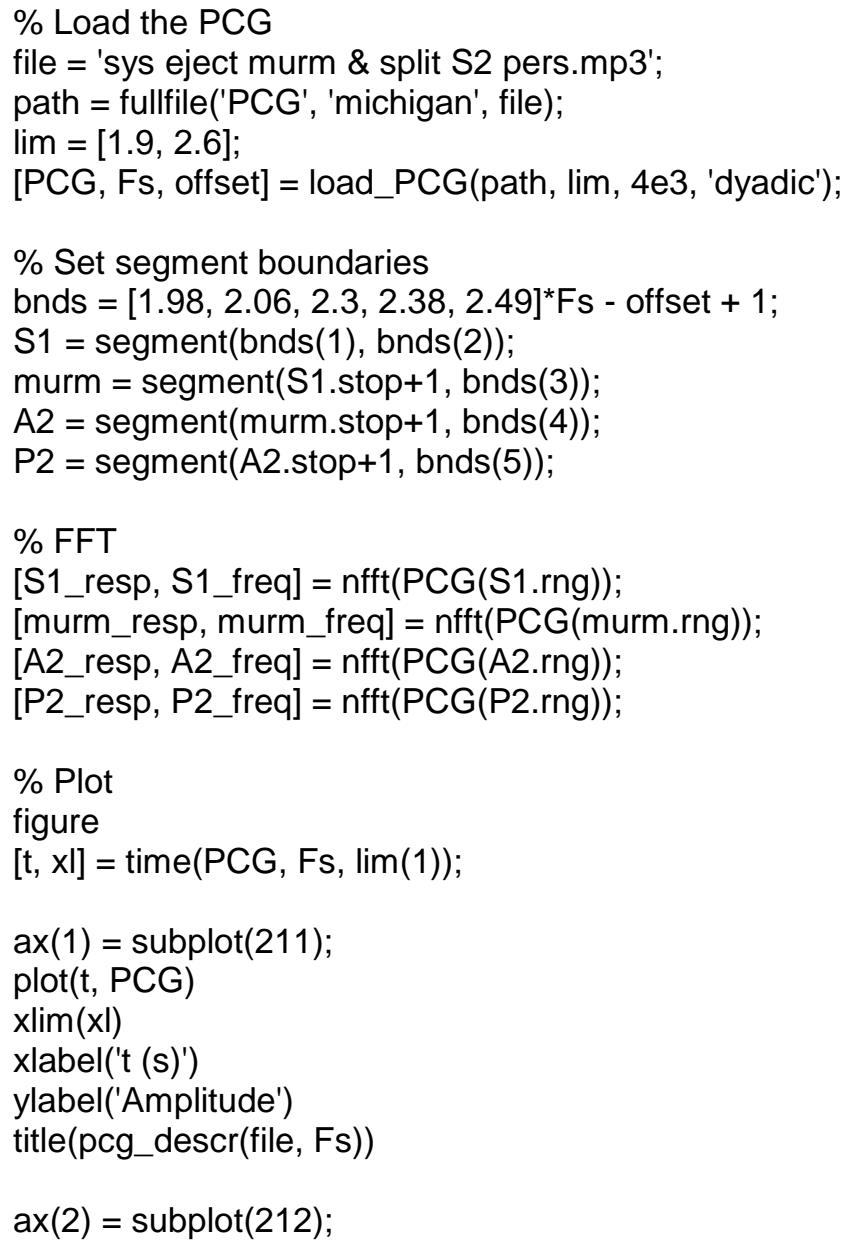




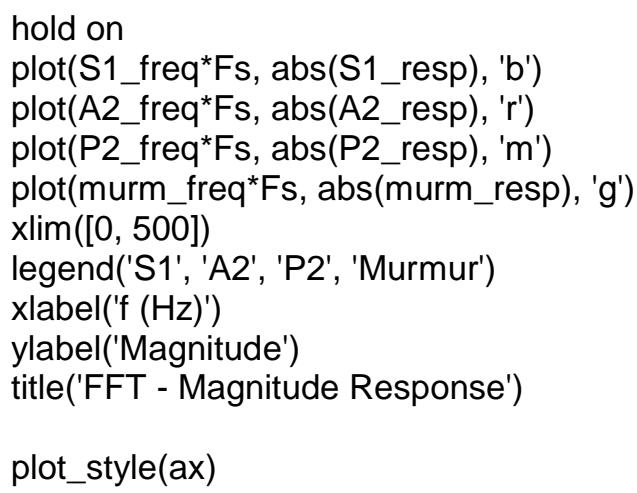

\section{A.1.5 PCG_simpl.m}

\% Demonstrate the simplicity transform of a PCG.

clear; clc; close all

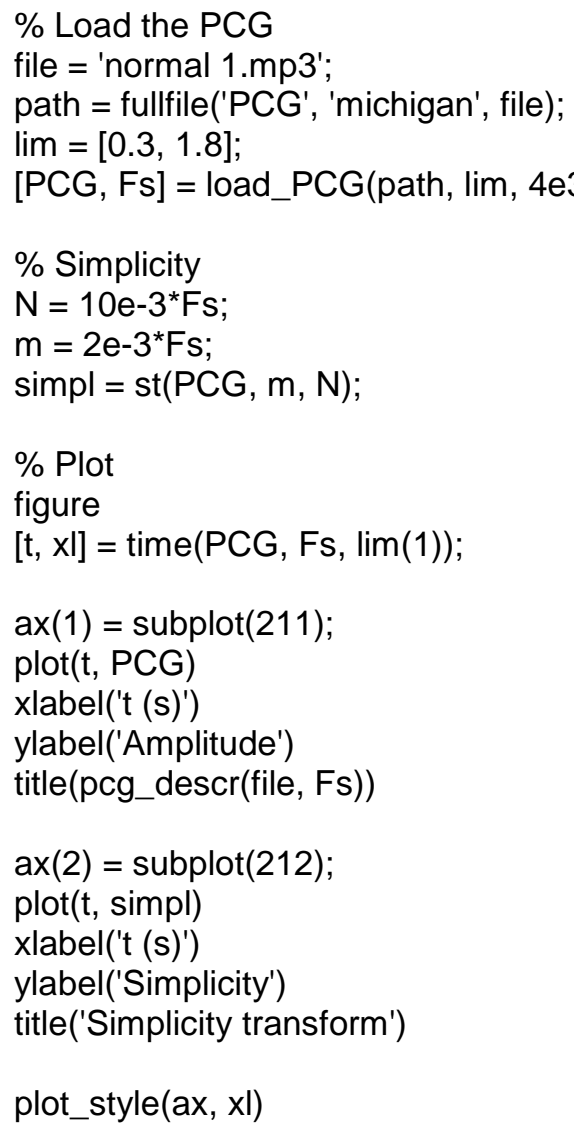

\section{A.1.6 rect_sinc.m}

$\%$ Compare rect $(\mathrm{t})$ and its Fourier transform $\operatorname{sinc}(\mathrm{f})$. clc; close all; clear

npts $=1000$

$\%$ time axis

$\mathrm{t} 1=-1$ 


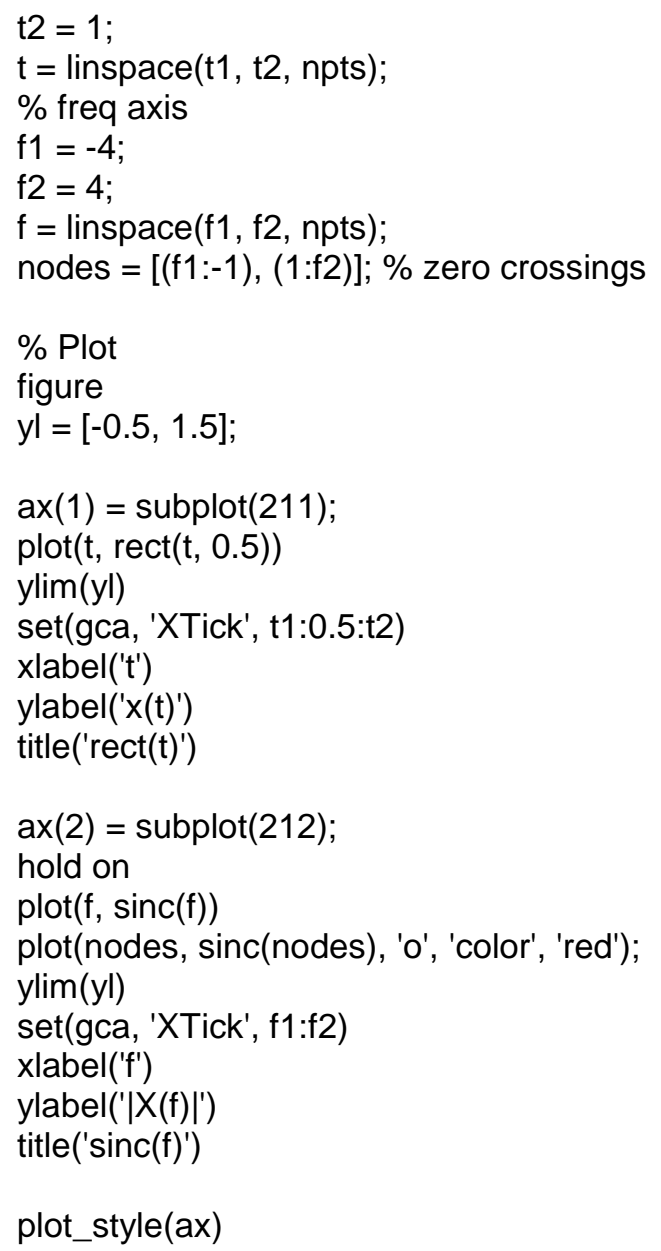

\section{A.1.7 singular_spectra.m}

$\%$ Compare the singular spectra of sinc and white gaussian noise.

clc; close all; clear

npts $=1000$

$\mathrm{t}=$ linspace(-10, 10, npts).';

$\%$ noise = "complex"

pwr = 0;

load $=50$;

noise $=$ wgn (npts, 1 , pwr, load, 'dBm');

$\%$ sinc = "simple"

$\sin \mathrm{c}=\sin \mathrm{c}(\mathrm{t})$;

$\%$ singular spectra

$\mathrm{m}=10$;

$\left[\sim, D \_\right.$noise $]=$st $($noise, $\mathrm{m})$;

$\left[\sim, D \_\right.$sinc $]=\operatorname{st}(\operatorname{sinc}, \mathrm{m})$;

$\%$ Plot

figure

$\%$ Signals

$a x(1)=\operatorname{subplot}(221)$; 


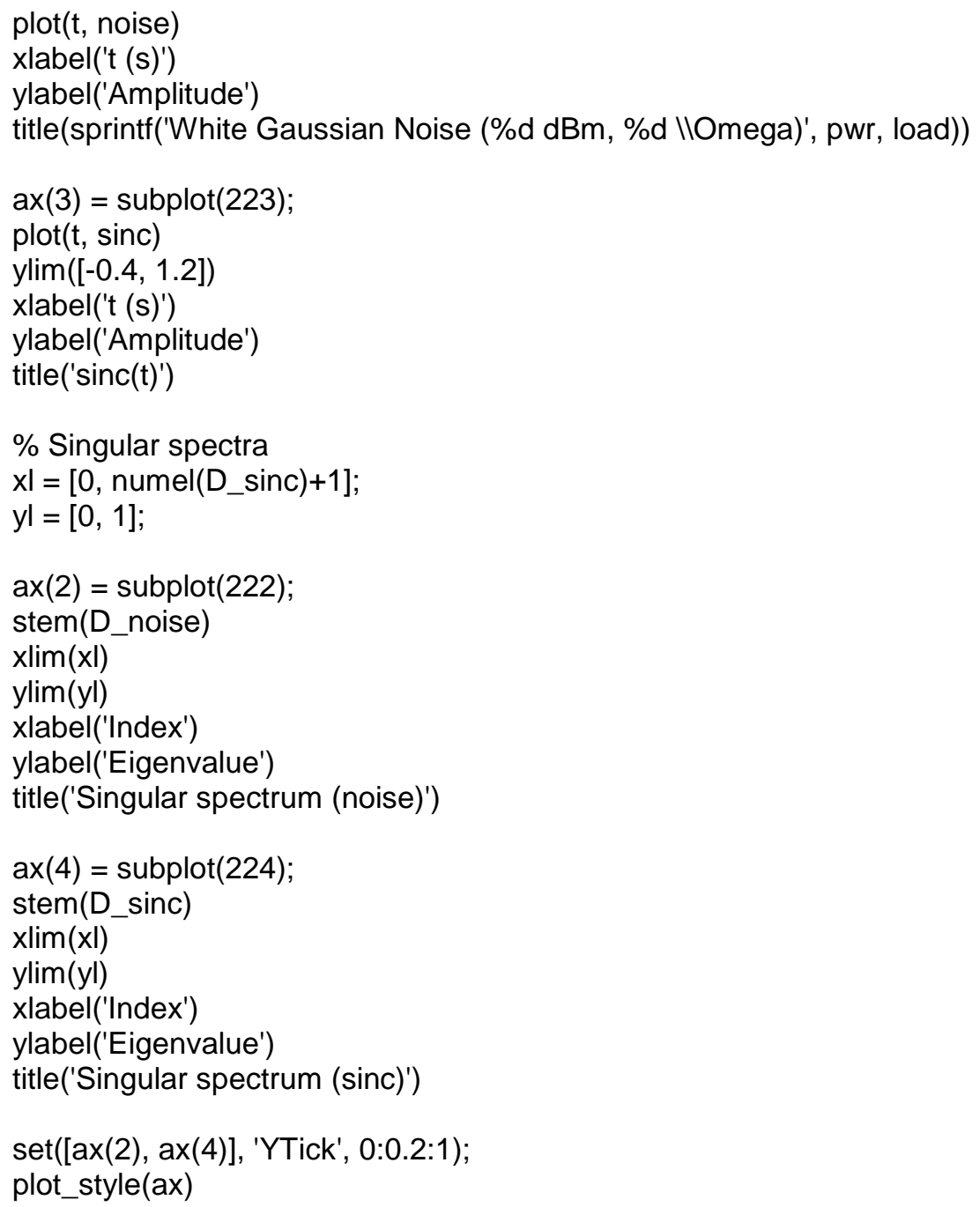

\section{A.2 Results}

\section{A.2.1 batch.m}

$\%$ Batch segment all sound files clear; close all; clc

root = 'C:IUsers $\backslash$ Josh $\backslash$ Google Drivelthesis $\backslash$ matlab';

Ivl1 = 2;

$|\mathrm{v}| 2=5$

$\% \%$ DWT

$\%$ Michigan

dwt =@(s) dwt_segment(s, Ivl2);

batch_segment(dwt, root, 'michigan', Ivl1)

\% Littmann

$\mathrm{dwt}=@(\mathrm{~s})$ dwt_segment(s, Ivl2, 'HS_thresh', 0.05);

batch_segment(dwt, root, 'littmann', Ivl1)

$\% \%$ Simplicity 
$\%$ Michigan

simpl =@(s) simpl_segment(s);

batch_segment(simpl, root, 'michigan', Ivl1)

$\%$ Littmann

simpl = @(s) simpl_segment(s);

batch_segment(simpl, root, 'littmann', Ivl1)

\section{A.2.2 beamforming.m}

$\%$ Plot the directivity pattern of a non-uniformly spaced planar stethoscope $\%$ array after beamforming.

clear; clc; close all

dir = 'C:IUsers $\backslash J o s h \backslash G o o g l e ~ D r i v e l t h e s i s \backslash m a t l a b|f i g u r e s \backslash c h p 2| ;$

$\mathrm{fmt}=$ 'epsc';

store $=$ false;

$\% \%$ Specify stethoscope locations

$\%$ Relative to the origin (inches)

$x=[-2.11,-2.03,1.91,2.25,0]$;

$y=[-0.938,1.29,0.887,-1.23,0]$;

$\mathrm{z}=\mathrm{zeros}(\operatorname{size}(\mathrm{y}))$;

$\%$ Convert to meters

$r=[x ; y ; z]^{*} 0.0254$;

$\% \%$ Plot stethoscope locations

fig = figure('color', 'white');

$\operatorname{plot}(x, y$, 'o', 'MarkerSize', 10, 'MarkerFaceColor', 'b')

xlabel(' $x$ ')

ylabel('y')

title('Relative stethoscope positions (meters)')

grid on

box on

$\%$ Save

if isempty(dir)

set(fig, 'PaperUnits', 'inches', 'PaperPosition', [0, 0, 6, 4])

saveas(gcf, fullfile(dir, 'stethoscope_locations'), fmt)

end

$\% \%$ Beamform

$\%$ Average speed of sound in tissue $(\mathrm{m} / \mathrm{s})$

$\mathrm{v}=1540$;

$\%$ Frequencies of interest

freq $(1)=500 ; \%$ innside heart murmur range

freq $(2)=7 e 3 ; \%$ outside of heart murmur range (no spatial aliasing)

freq $(3)=20 \mathrm{e} 3 ; \%$ outside of heart murmur range (spatial aliasing)

$\%$ Target angles

theta_t $=\mathrm{pi} / 2$;

phi_t $=(0: 1 / 6: 1){ }^{*}$ pi;

$\%$ Resolution

npts $=500$;

$\%$ Sweep

for $f=$ freq

fprintf('Frequency $=\% .0 \mathrm{e} \mathrm{Hz} \backslash n$ ', f)

isAliased(r, f, v, ' $m$ ', 3); \% check for spatial aliasing 


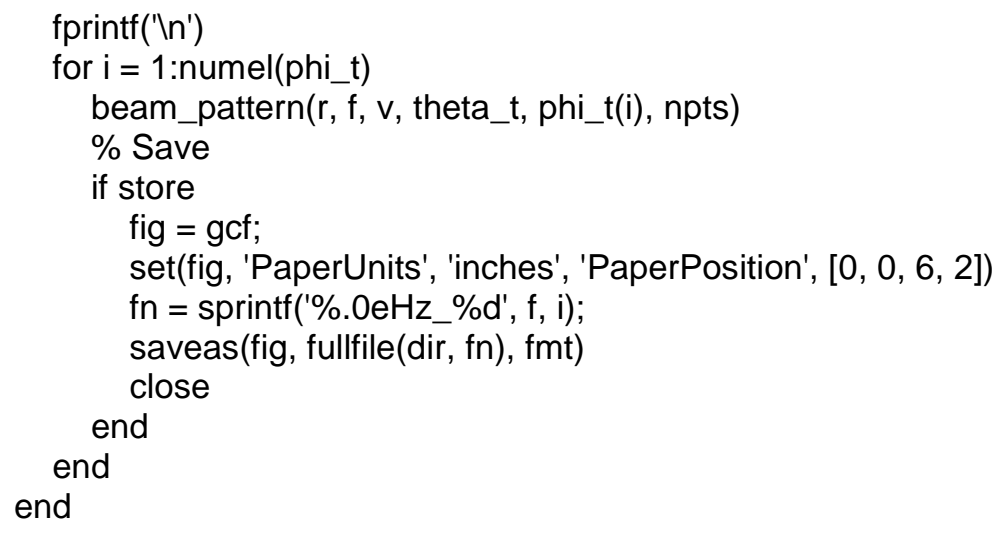

\section{A.2.3 dwt_michigan.m}

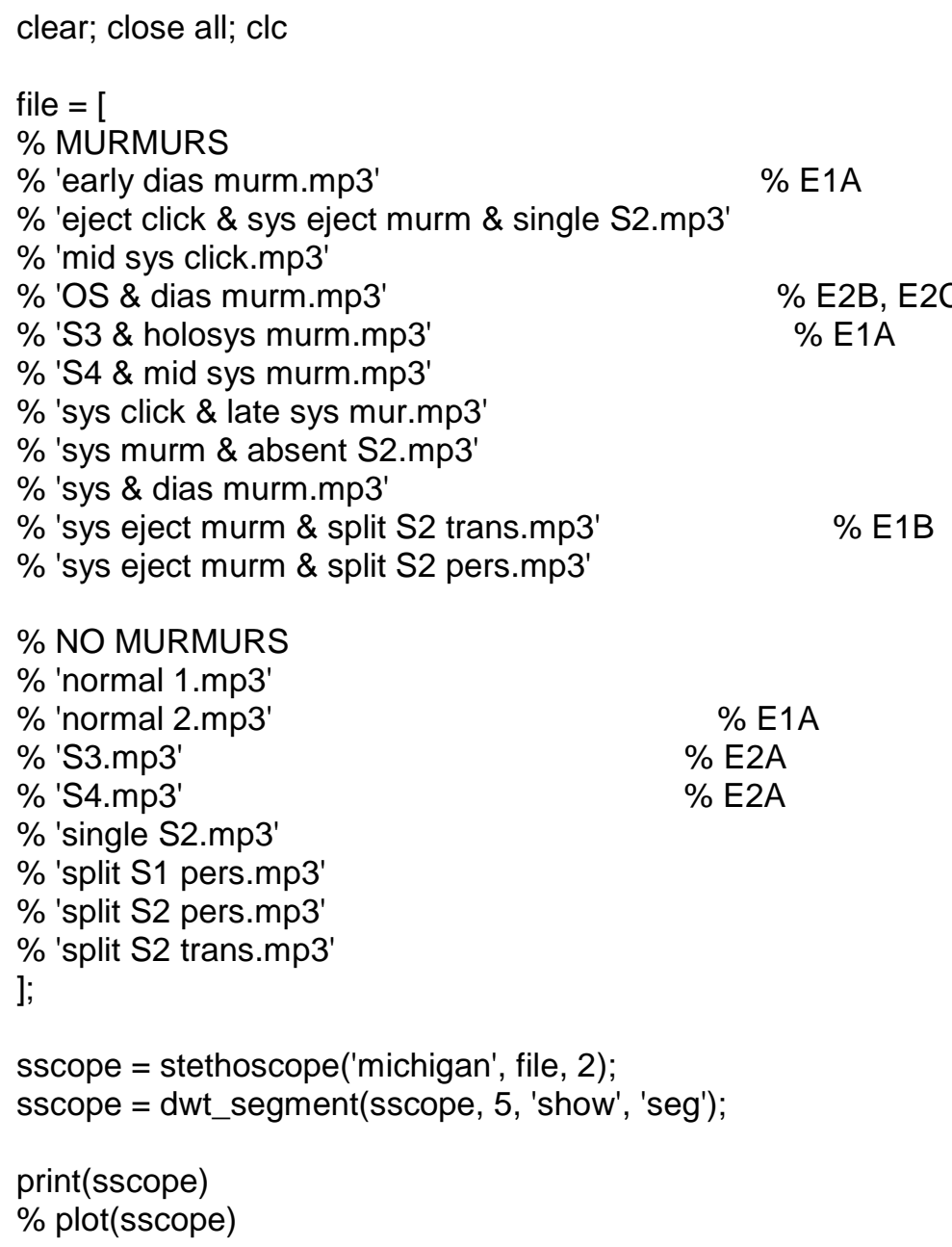

\section{A.2.4 dwt_littmann.m}

clear; close all; clc

file $=[$

$\%$ MURMURS 


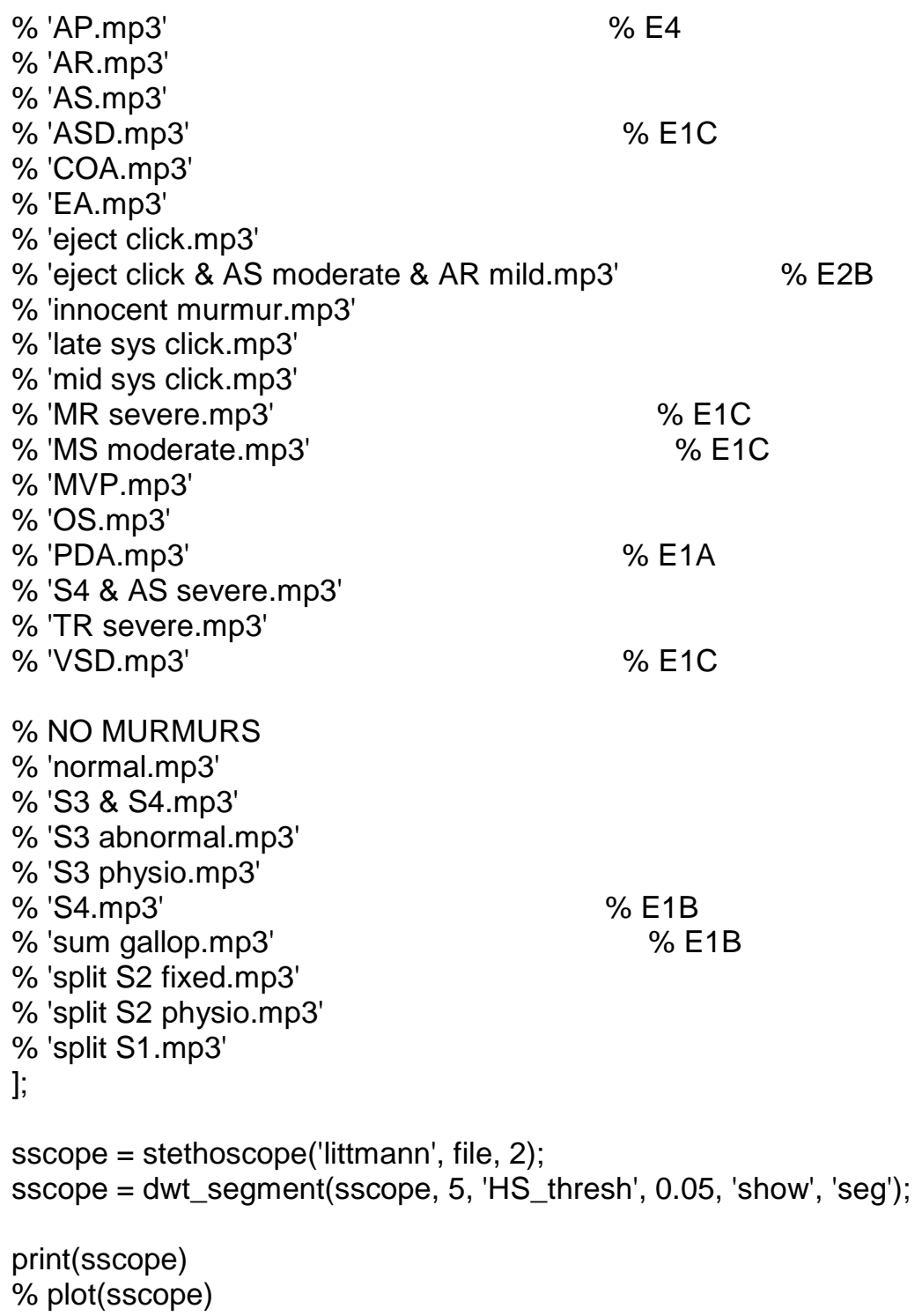

$\% \mathrm{E} 1 \mathrm{~B}$

$\%$ E1B

\section{A.2.5 simpl_michigan.m}

clear; close all; clc

file $=[$

$\%$ MURMURS

$\%$ 'early dias murm.mp3'

$\%$ 'eject click \& sys eject murm \& single S2.mp3'

$\%$ 'mid sys click.mp3'

$\%$ 'OS \& dias murm.mp3'

$\%$ 'S3 \& holosys murm.mp3'

$\%$ 'S4 \& mid sys murm.mp3'

$\%$ 'sys click \& late sys mur.mp3'

$\%$ 'sys murm \& absent S2.mp3'

$\%$ 'sys \& dias murm.mp3'

$\%$ 'sys eject murm \& split S2 trans.mp3'

$\%$ 'sys eject murm \& split S2 pers.mp3' 


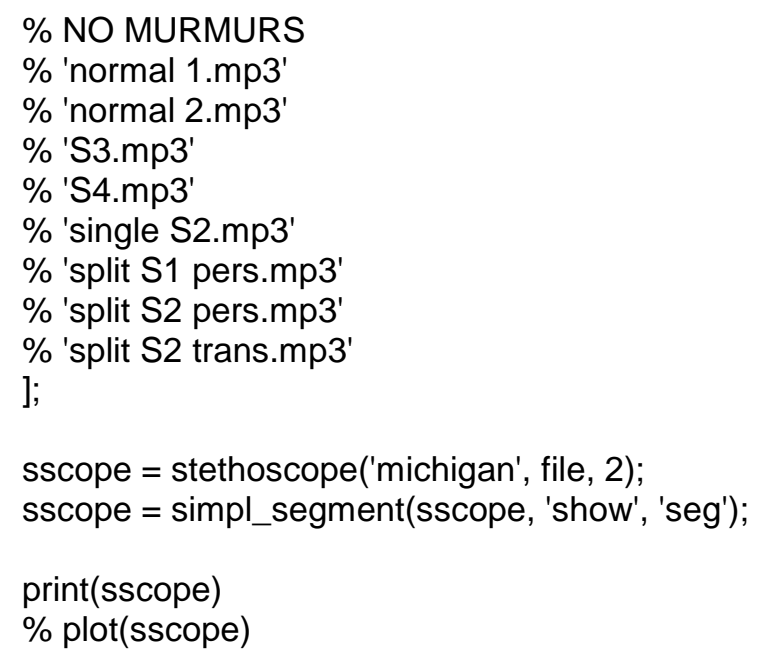

\section{A.2.6 simpl_littmann.m}

clear; close all; clc

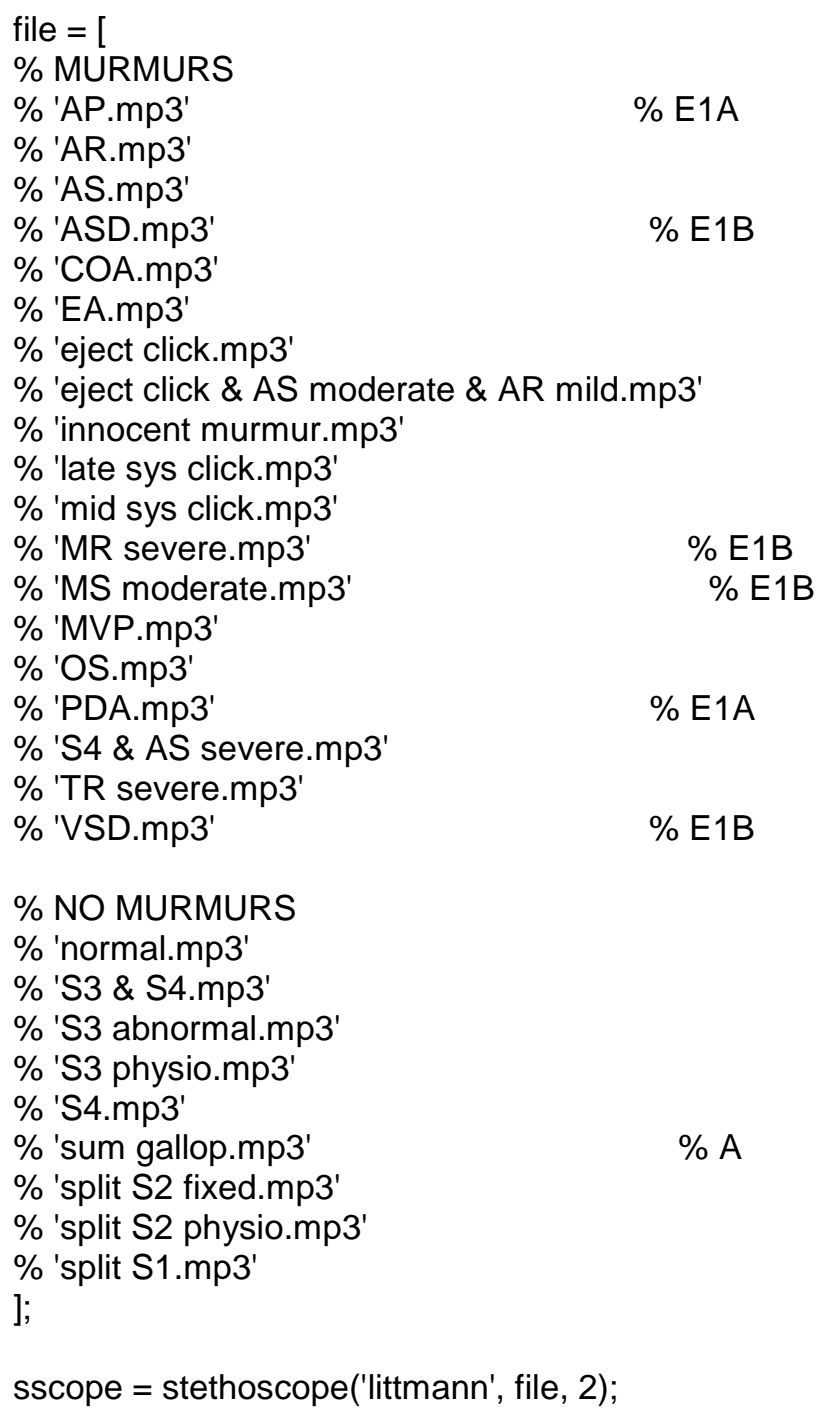


sscope = simpl_segment(sscope, 'show', 'seg');

print(sscope)

$\%$ plot(sscope) 


\section{B. Functions}

\section{B.1 Beamforming}

\section{B.1.1 beam_pattern.m}

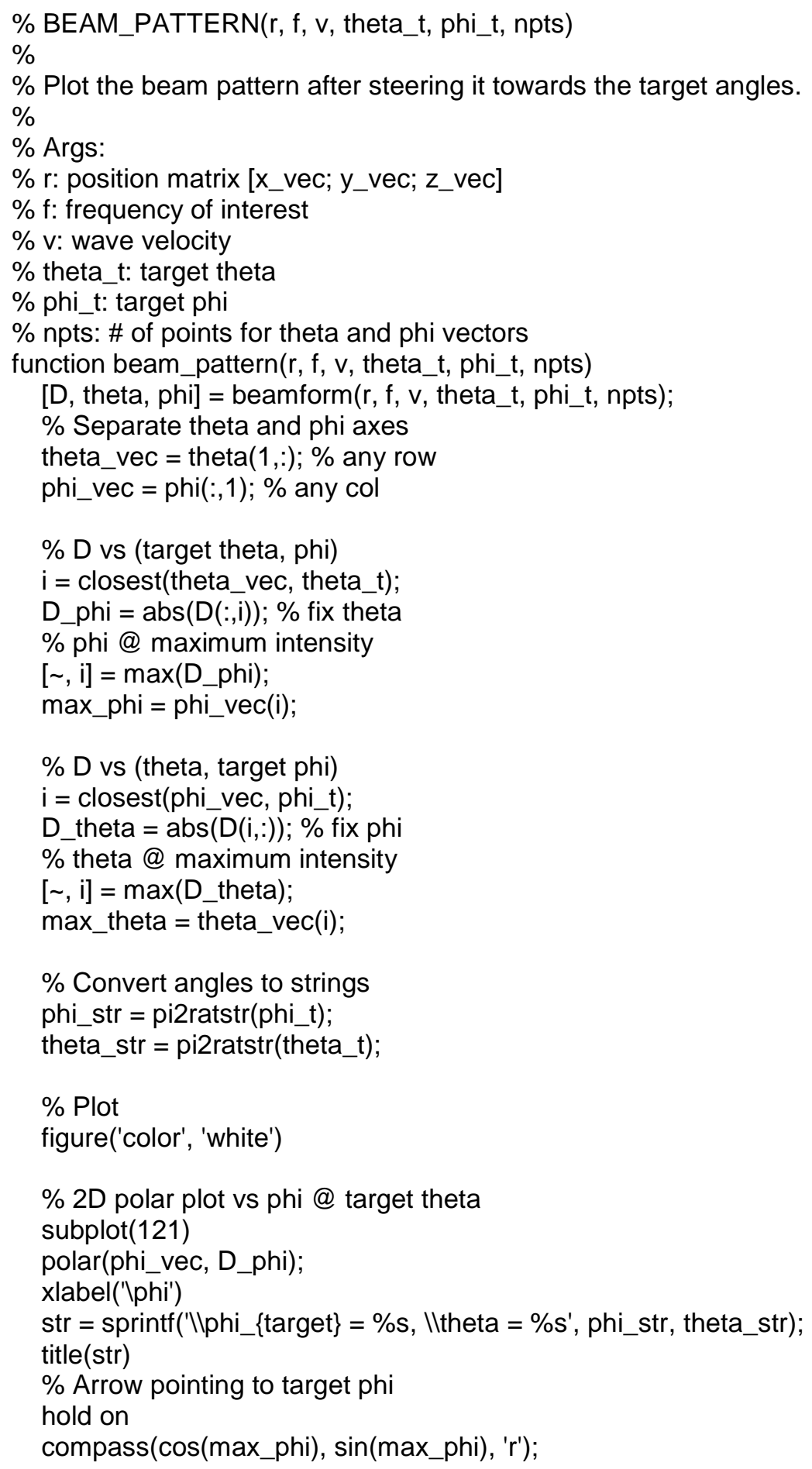




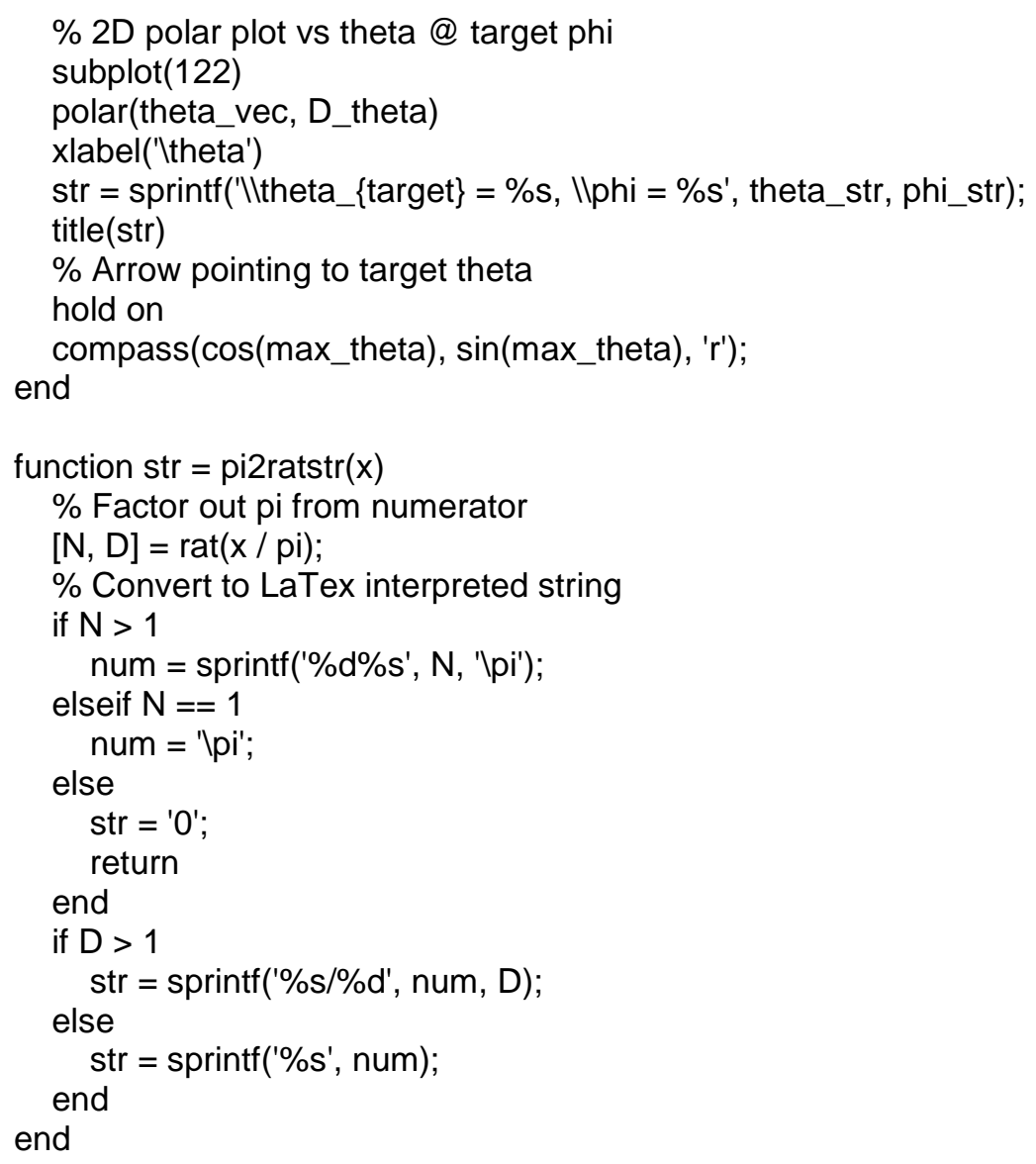

\section{B.1.2 beamform.m}

$\%[D$, theta, phi $]=$ BEAMFORM $(r, f, v$, theta_t, phi_t $t$ npts $)$

$\%$

$\%$ Steer the beam pattern towards the target angles by applying an angular

$\%$ dependent phase weight to each sensor.

$\%$

$\%$ Args:

$\%$ r: position matrix [x_vec; y_vec; z_vec]

$\%$ f: frequency of interest

$\%$ v: wave velocity

$\%$ theta_t: target theta

$\%$ phi t: target phi

$\%$ npts: \# of points for theta and phi vectors

$\%$

$\%$ Returns:

$\%$ D: directivity pattern (matrix)

$\%$ theta: polar angles (matrix)

$\%$ phi: azimuthal angles (matrix)

function $[D$, theta, phi] $=$ beamform $(r, f, v$, theta_t $t$ phi_t, npts $)$

$\mathrm{f}=\operatorname{abs}(\mathrm{f})$;

$\mathrm{v}=\operatorname{abs}(\mathrm{v})$;

$\%$ theta $=$ row, phi $=$ column

gv = linspace $\left(0,2^{*} \mathrm{pi}\right.$, npts $)$;

[theta, phi] $=$ meshgrid $(\mathrm{gv})$; 


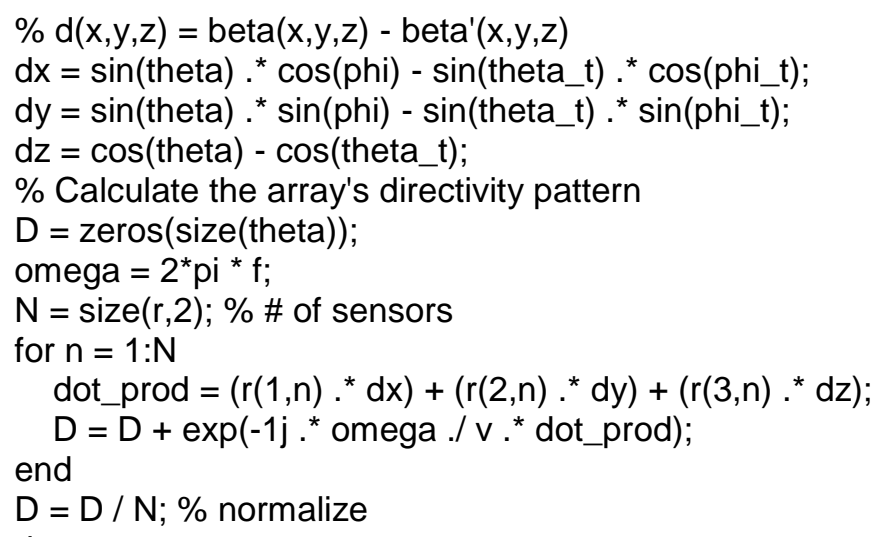

\section{B.1.3 dist_mat.m}

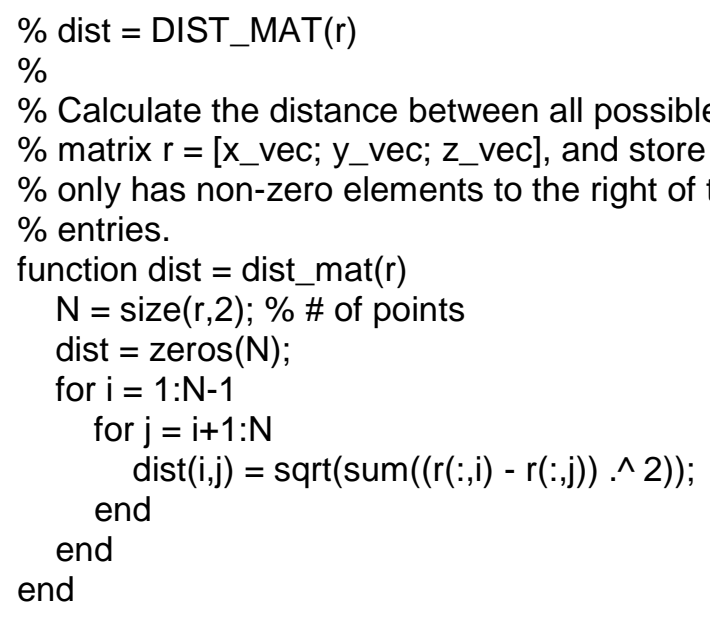

\section{B.1.4 isAliased.m}

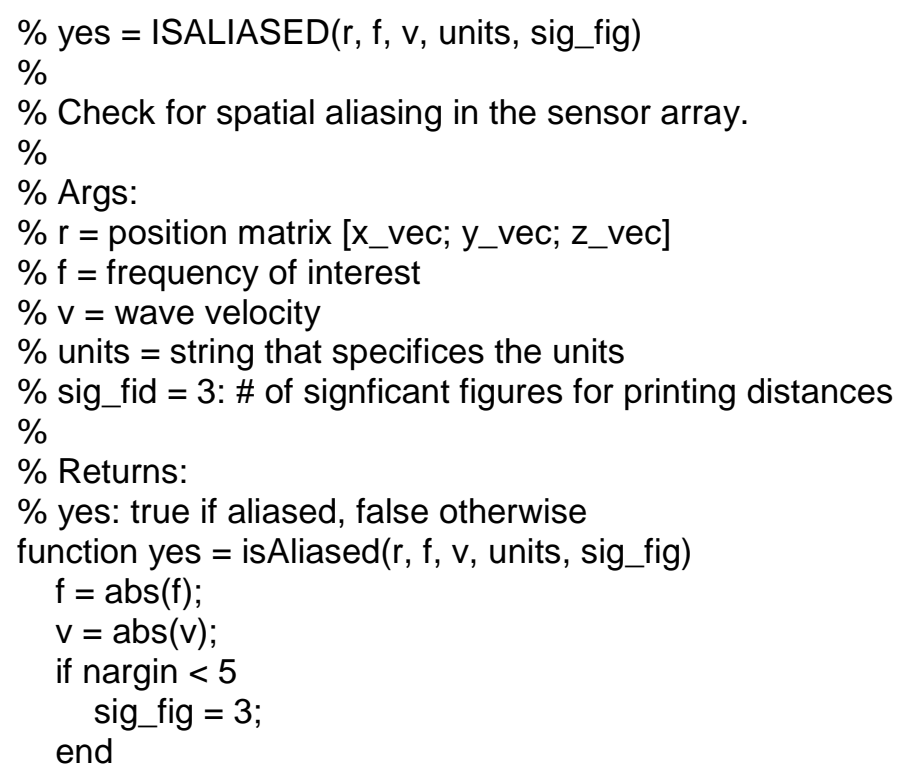




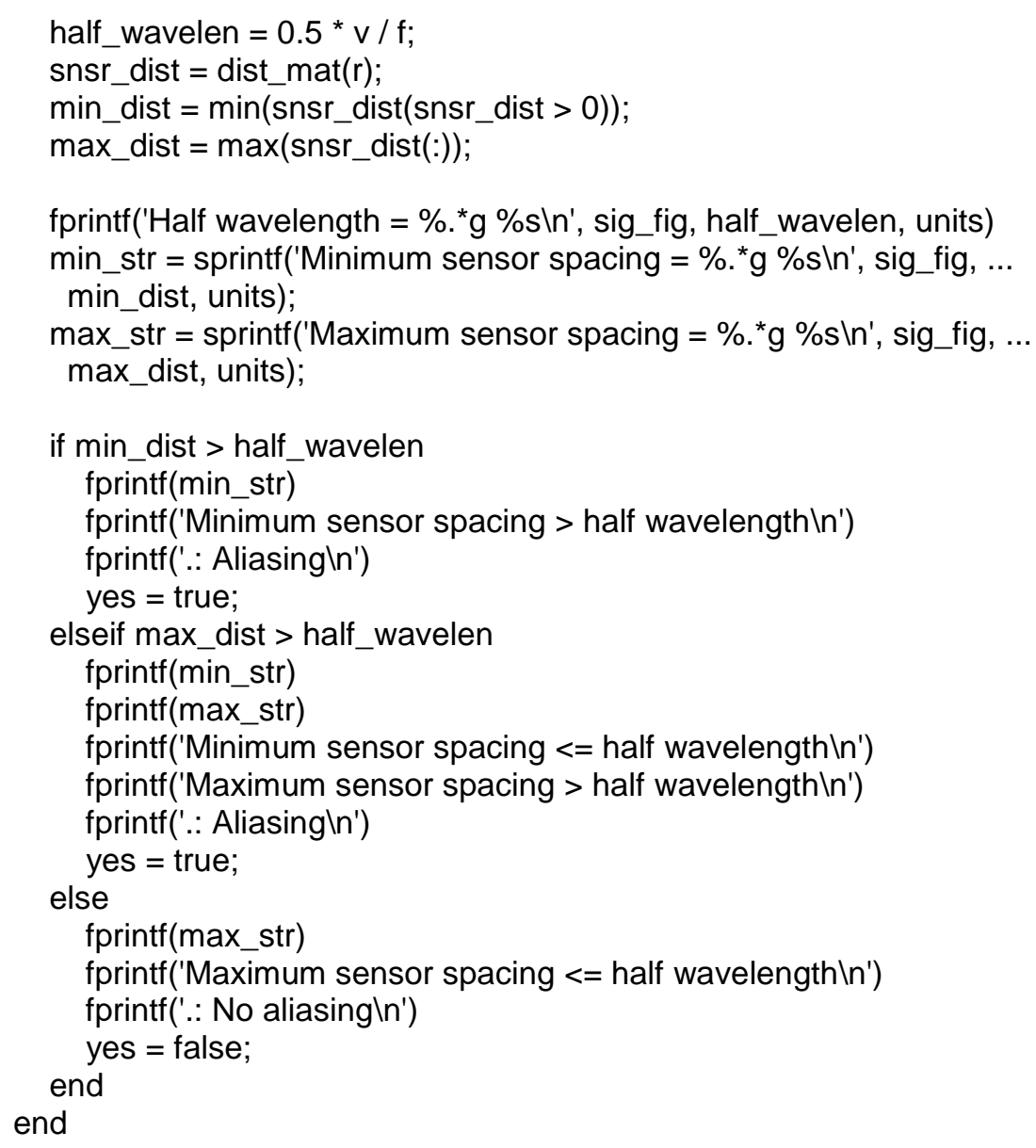

\section{B.2 DWT}

\section{B.2.1 coef_plot.m}

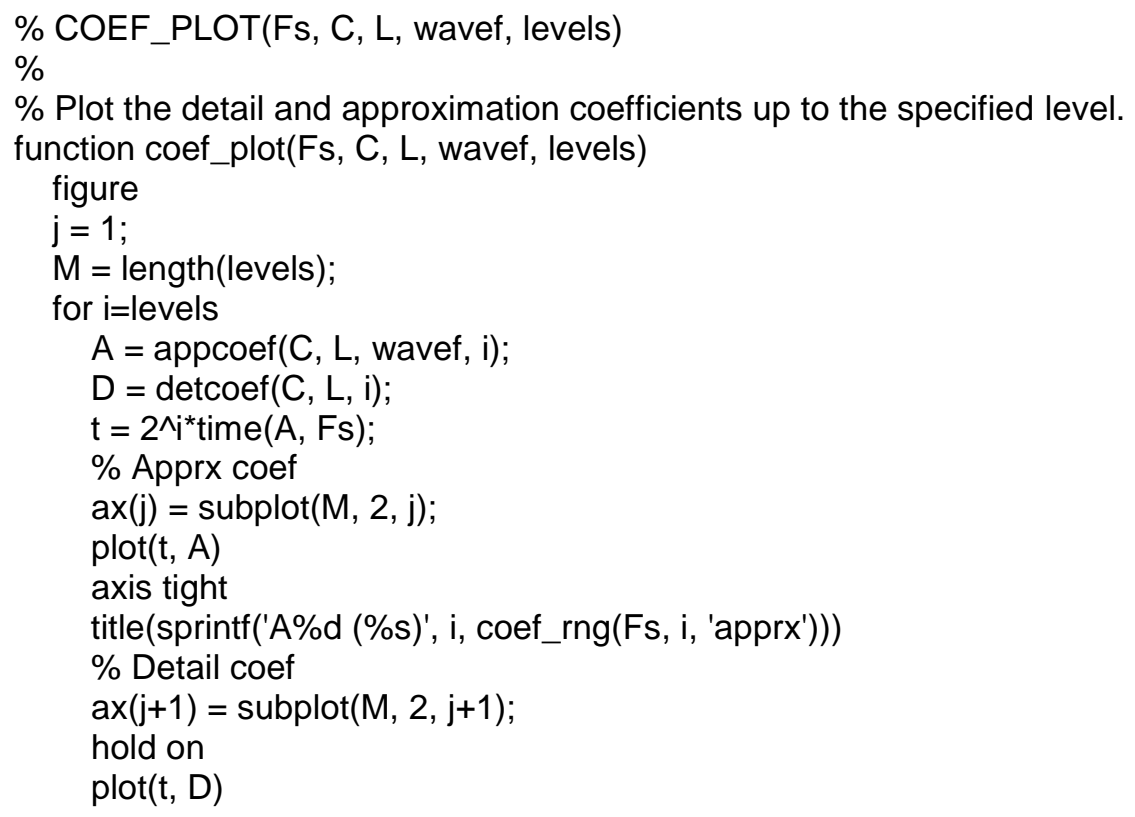




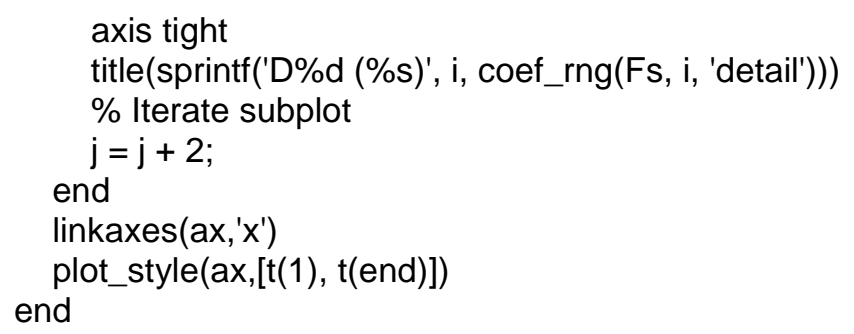

\section{B.2.2 coef_rng.m}

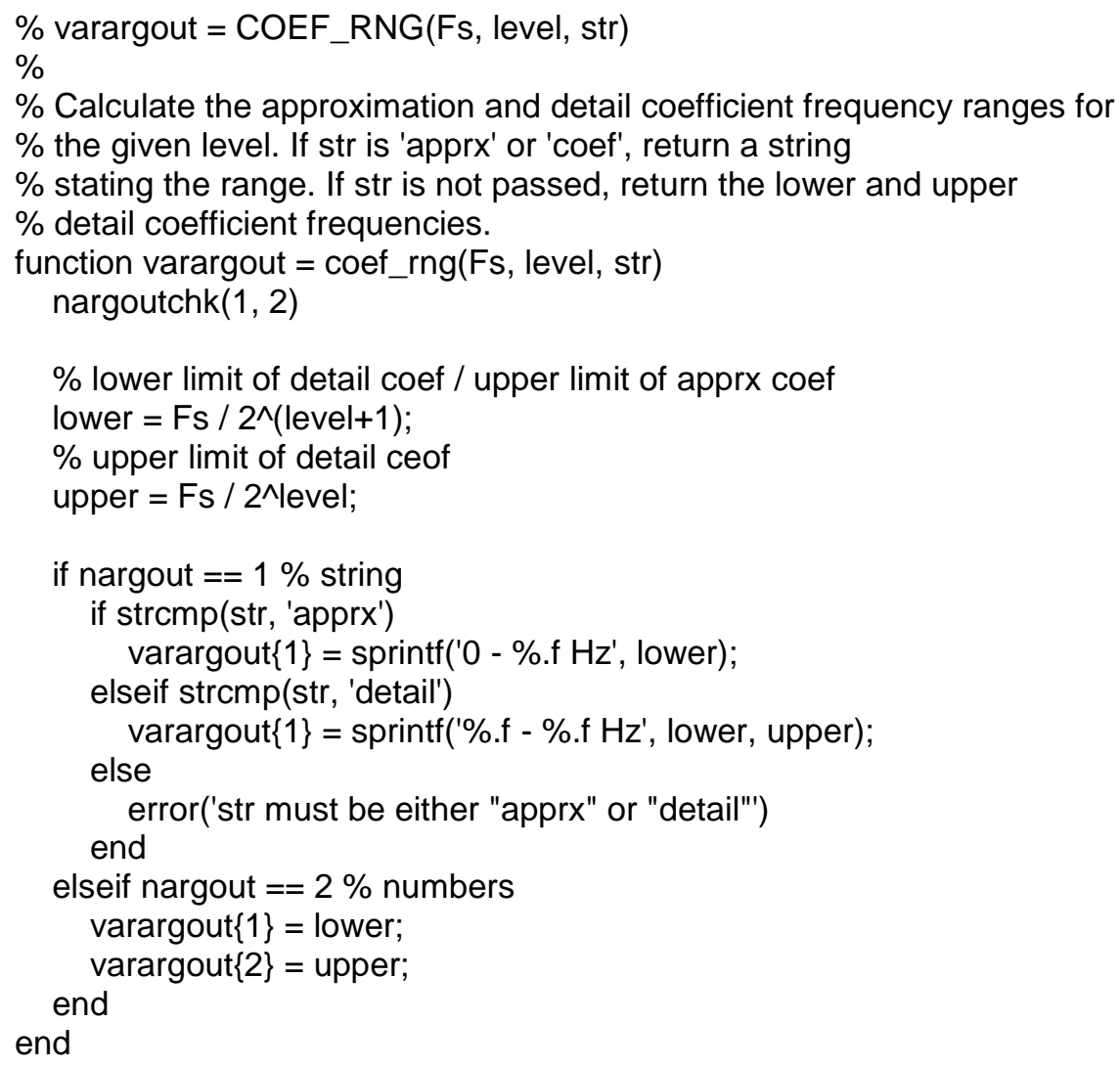

\section{B.3 Main}

\section{B.3.1 batch_segment.m}

$\%$ BATCH_SEGMENT(seg_func, root, folder, varargin)

$\%$

$\%$ Segment all sound files in /root/PCG/folder with the segmentation function

$\%$ seg_func. The varargins are inputs to the @stethoscope constructor.

function batch_segment(seg_func, root, folder, varargin)

pdir = fullfile(root, 'PCG', folder);

list $=\operatorname{dir}($ pdir);

files $=\{$ list $(3:$ end $)$. name $\}$;

for $\mathrm{i}=1$ :numel(files)

file $=$ files $\{$ i $;$

sscope $=$ stethoscope (folder, file, varargin $\{:\})$; 


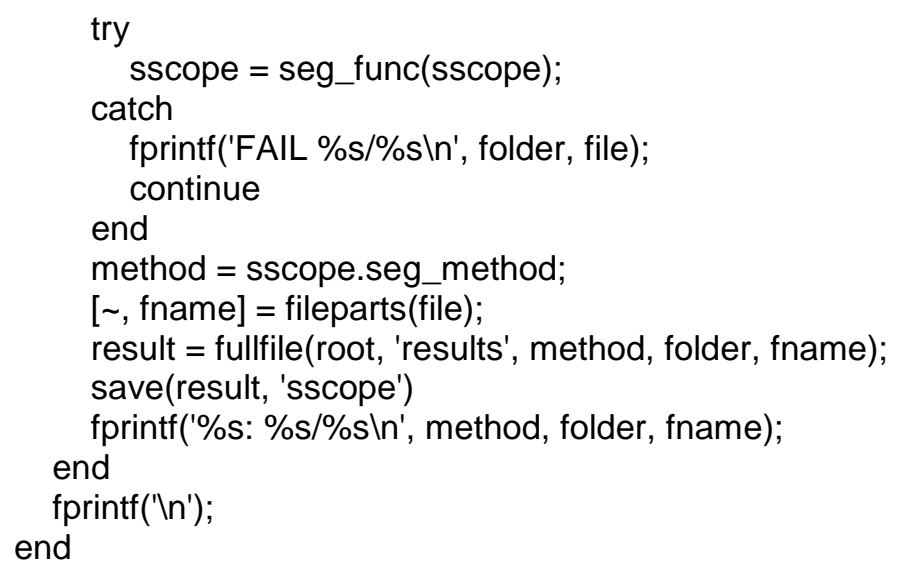

\section{B.3.2 find_heart_cycles.m}

$\%$ cyc_bnds = FIND_HEART_CYCLES(HS, PCG, min_dist, show, Fs)

$\%$

\% Locate the heart cycle boundaries from peaks in the PCG envelope's

$\%$ autocorrelation waveform.

$\%$

$\%$ Args:

$\%$ * HS: heart sound segments

$\%$ *PCG

$\%$ * min_dist: minimum distance between heart cycles, which is typically twice

$\%$ the minimum systole duration

$\%{ }^{*}$ show = false: show the plots if true

$\%{ }^{*} \mathrm{Fs}=1$ : sampling rate for plotting

function cyc_bnds = find_heart_cycles(HS, PCG, min_dist, show, Fs)

if isempty( $\mathrm{HS})$

error('At least one heart sound segment is required.')

end

min_dist $=$ abs (min_dist);

if nargin $<4$

show $=$ false;

end

if nargin $<5$

$$
\mathrm{Fs}=1
$$

else

$\mathrm{Fs}=\mathrm{abs}(\mathrm{Fs})$;

end

$\%$ Retrieve relative cycle bounds ( $\mathrm{N}$ bounds $=\mathrm{N}$ cycles here)

[cyc_bnds, ax] = find_cycles(PCG, min_dist, show, Fs);

if isempty(cyc_bnds)

error('No cycle boundaries detected.') end

$\%$ Move the bounds near HS.strt

$\%$ Add another bound at the 1st HS and shift the other bounds accordingly offset $=\mathrm{HS}(1)$.strt;

cyc_bnds = [offset, offset + cyc_bnds];

$\%$ Remove any bounds that now exceed the PCG duration

cyc_bnds(cyc_bnds > length(PCG) $=$ [];

if length(cyc_bnds) $<2$ 


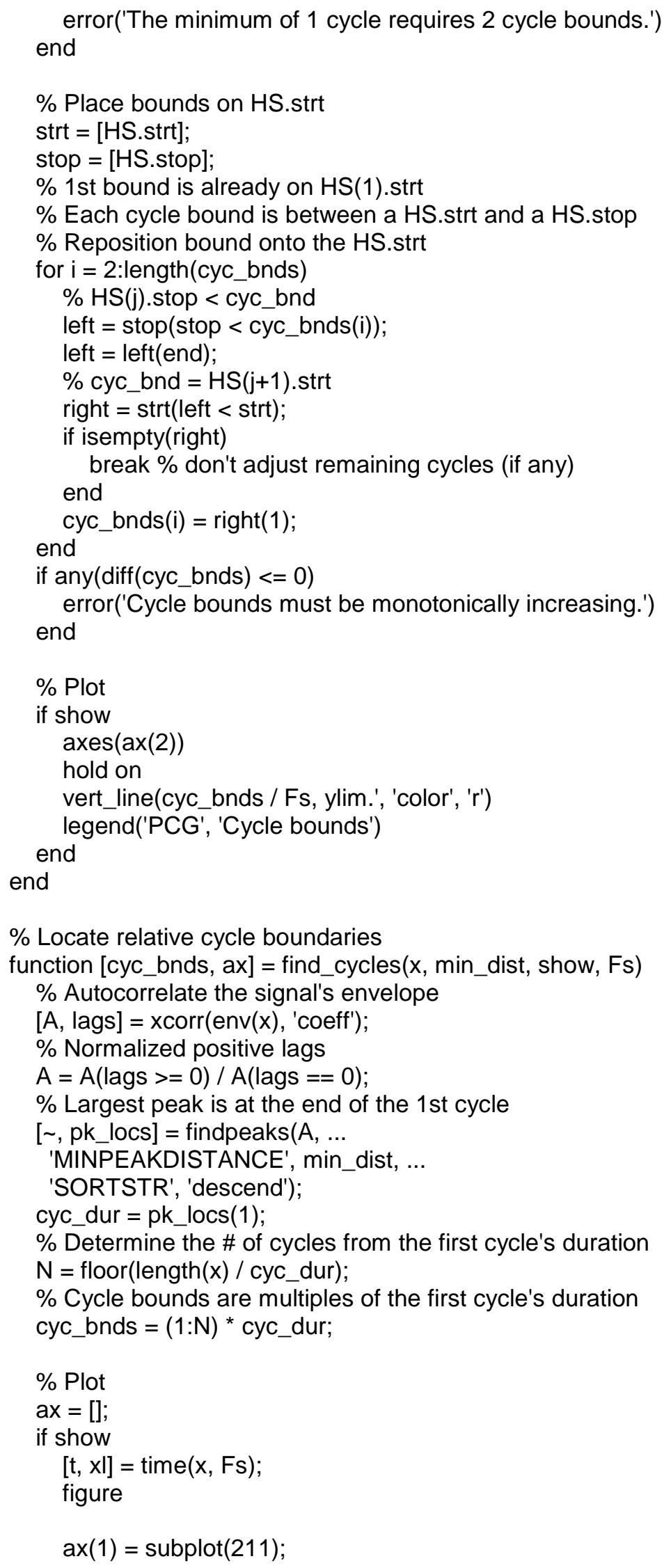




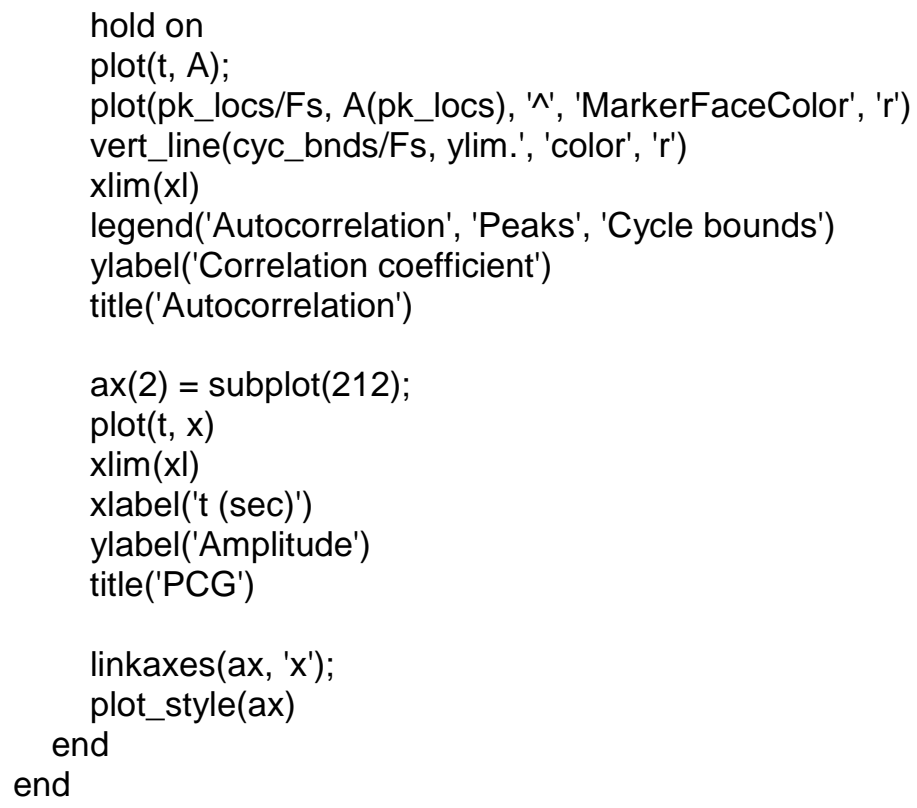

\section{B.3.3 katz_fd.m}

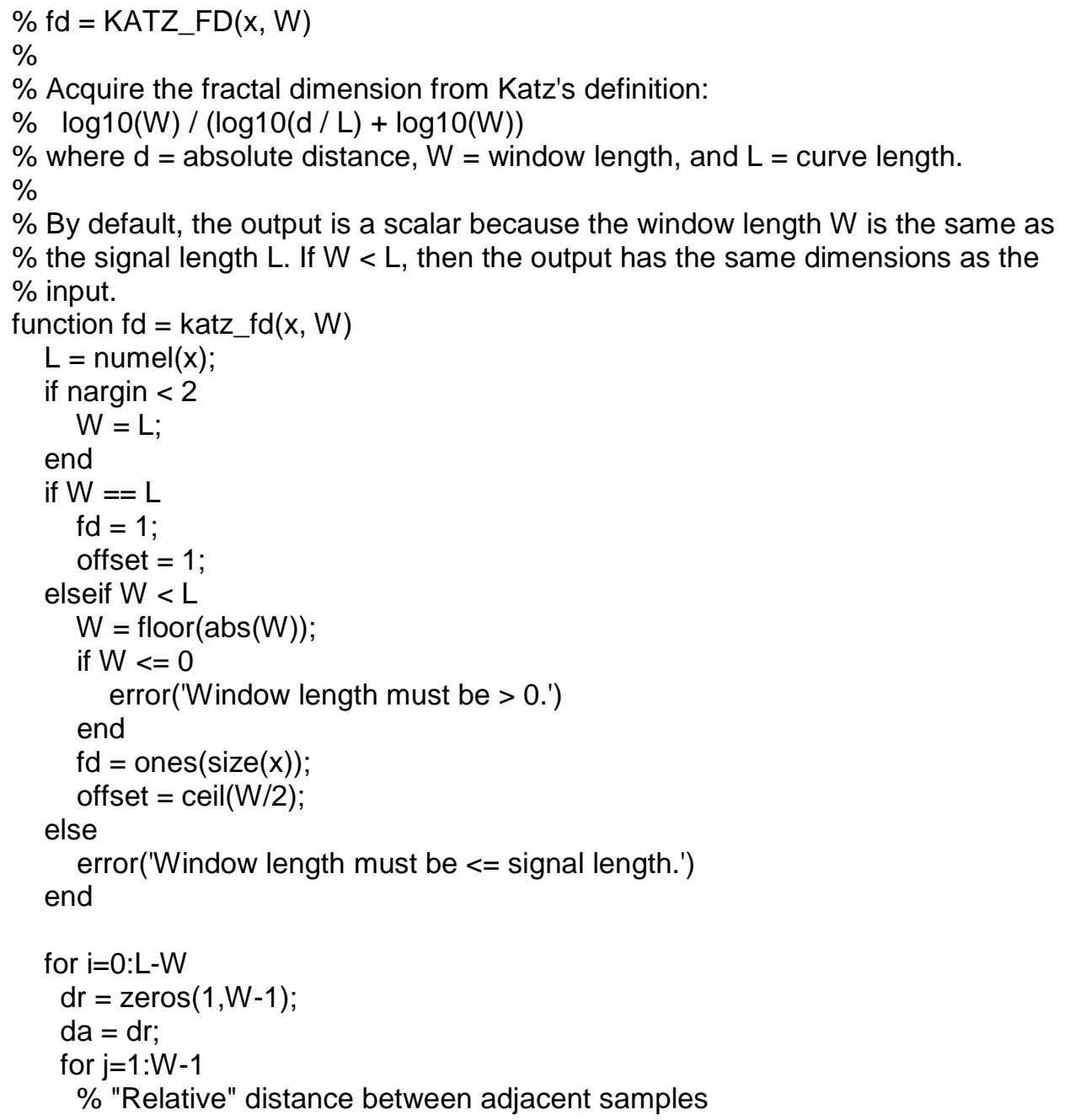




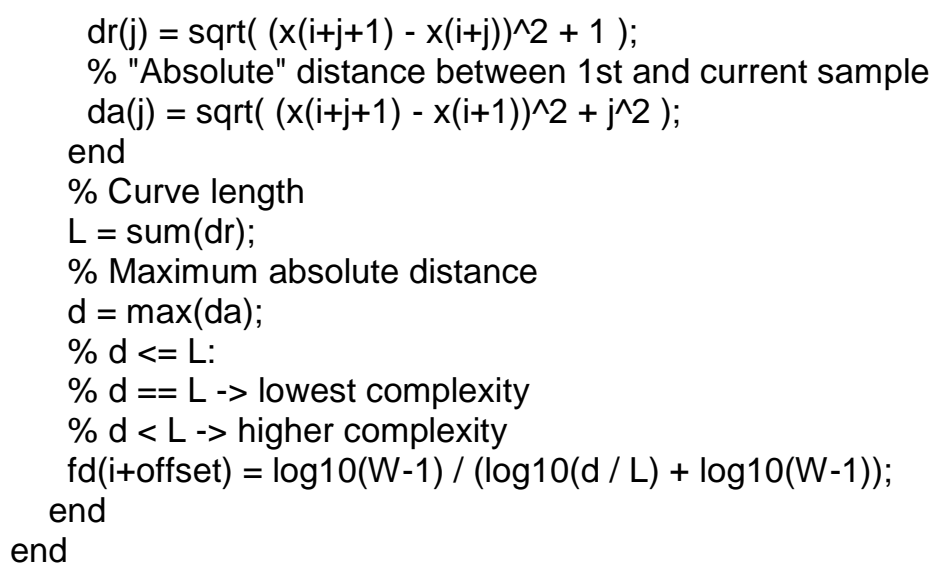

\section{B.3.4 lbl_sounds.m}

$\%[S 1, \mathrm{M} 1, \mathrm{~T} 1, \ldots$

$\% \mathrm{~S} 2, \mathrm{~A} 2, \mathrm{P} 2, \ldots$

$\%$ S3, S4, sum_gallop,...

$\%$ syst_murm, diast_murm] = LBL_SOUNDS(HS, cHS, cyc_bnds, extra_HS, murm)

$\%$

$\%$ Classify the normal heart sounds, extra heart sounds, and murmurs as specific

$\%$ sound types.

$\%$

$\%$ The systole and diastole segments are identified by their relative durations

$\%$ within each cycle (systole is shorter than diastole), which makes it possible

$\%$ to classify the normal heart sounds as S1 or S2. Then, S1 and S2 are searched

$\%$ for split sound segments, systole and diastole are searched for murmurs, and

$\%$ diastole is also searched for extra heart sounds. Finally, each segment is

$\%$ stored in an array that corresponds to its specific sound type, namely: S1 or

$\%$ S2 for normal heart sounds; M1 or T1 for split S1 components; A2 or P2 for

$\%$ split S2 components; S3, S4, or sum_gallop for extra heart sounds; and

$\%$ syst_murm for systolic murmurs and diast_murm for diastolic murmurs.

$\%$

$\%$ Args:

$\%{ }^{*} \mathrm{HS}$ : normal heart sound segments

$\%{ }^{*} \mathrm{cHS}$ : normal heart sound segments except split sound segments are combined

$\%$ * cyc_bnds: heart cycle boundaries

$\%$ * extra_HS = segment.empty: S3, S4, or summation gallop segments

$\%{ }^{*}$ murm = segment.empty: murmur segments

$\%$

$\%$ Returns:

$\%$ * $S 1$... sum_gallop: segment array [1, \# heart cycles]

$\%$ * syst_murm, diast_murm: cell array [1, \# heart cycles], where each cell

$\%$ contains a variable length segment array since there can be multiple murmur

$\%$ segments in systole or diastole

function [S1, M1, T1, S2, A2, P2, S3, S4, sum_gallop, syst_murm, diast_murm] = Ibl_sounds(...

HS, cHS, cyc_bnds, extra_HS, murm)

cyc_bnds $=\overline{\text { floor}}($ abs $($ cyc_bnds $))$;

if nargin $<4$

end

extra_HS = segment.empty;

if nargin $<5$ end

murm = segment.empty; 


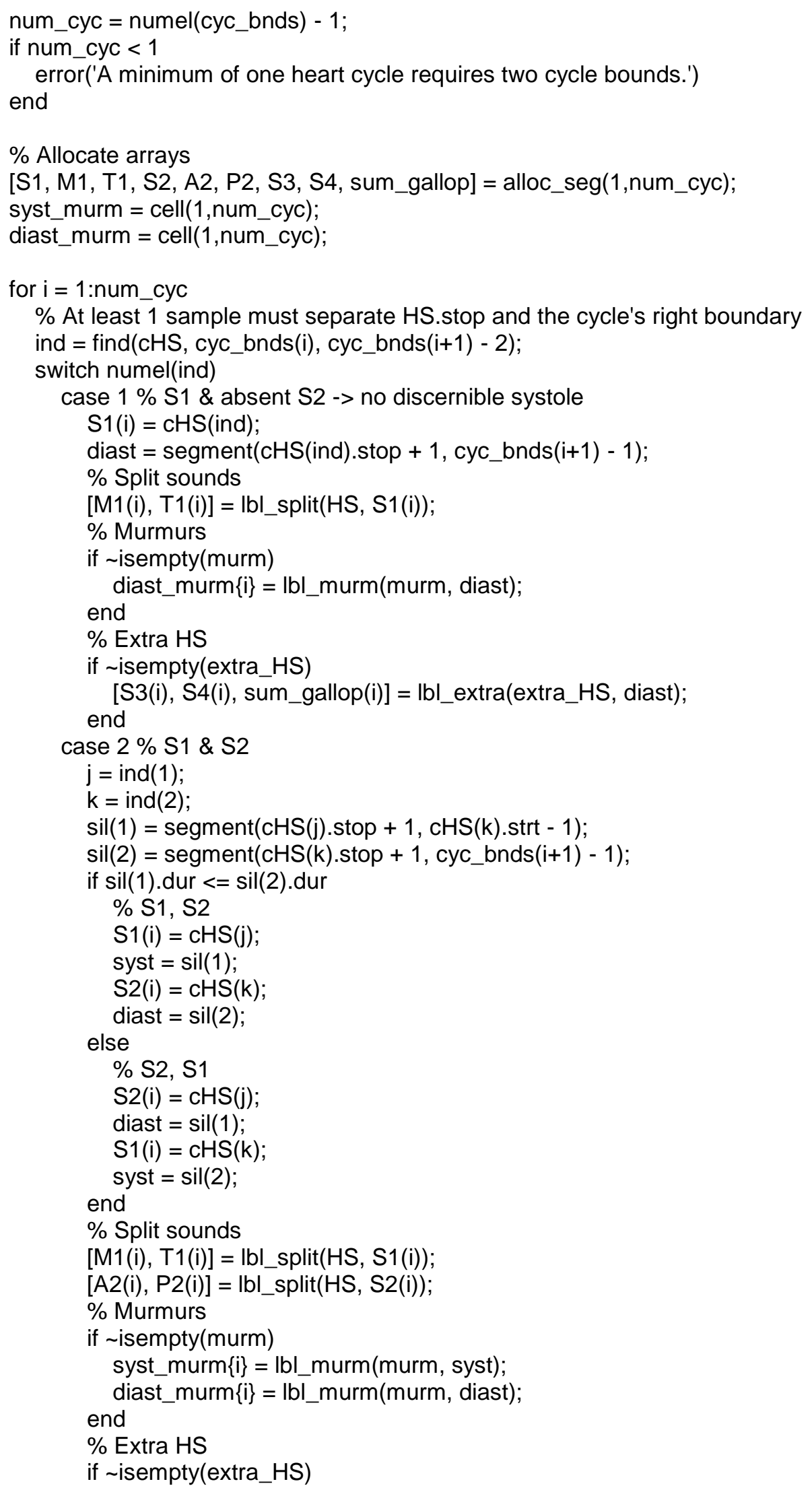




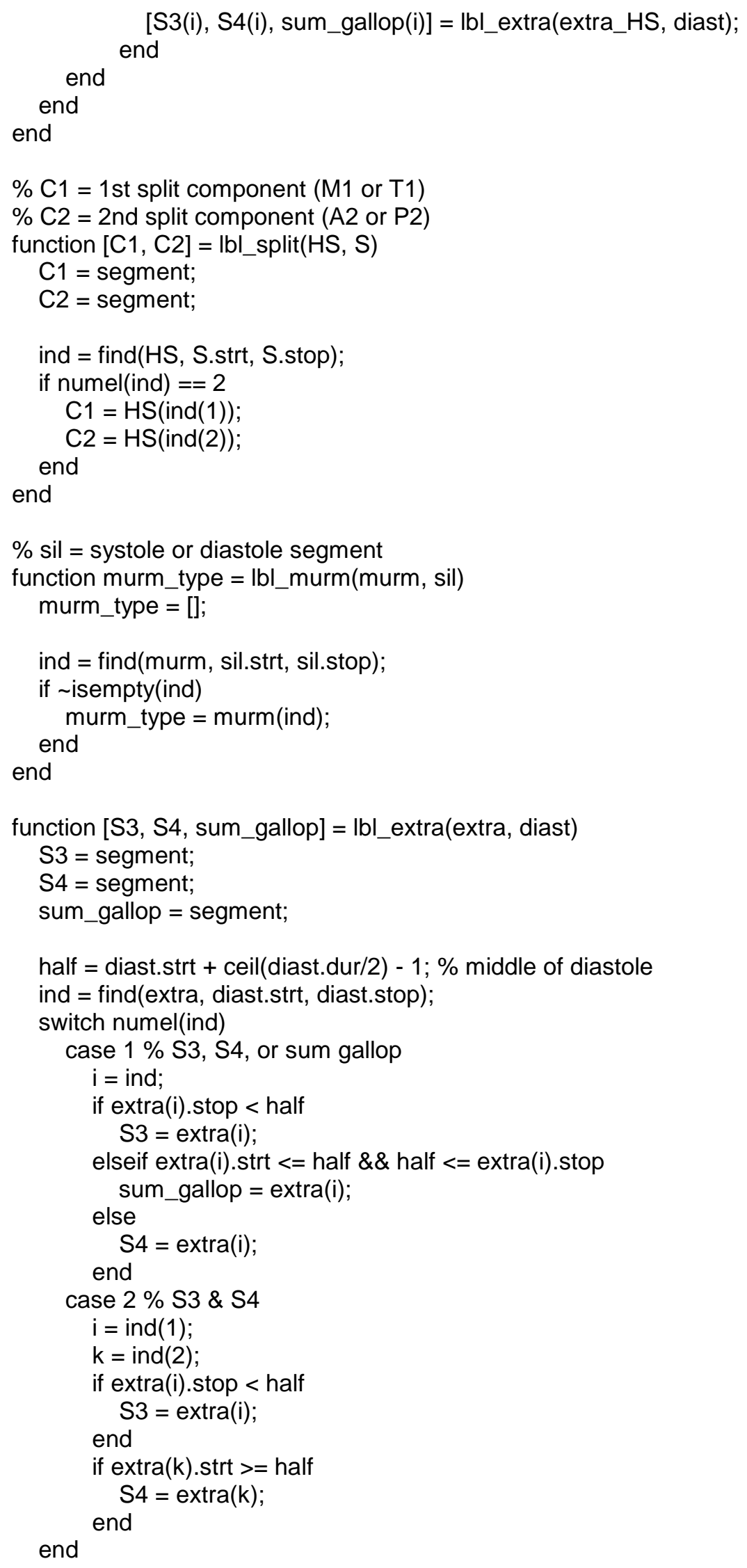




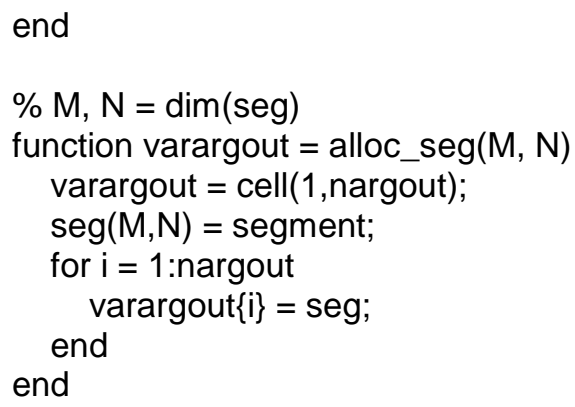

\section{B.3.5 levels2seg.m}

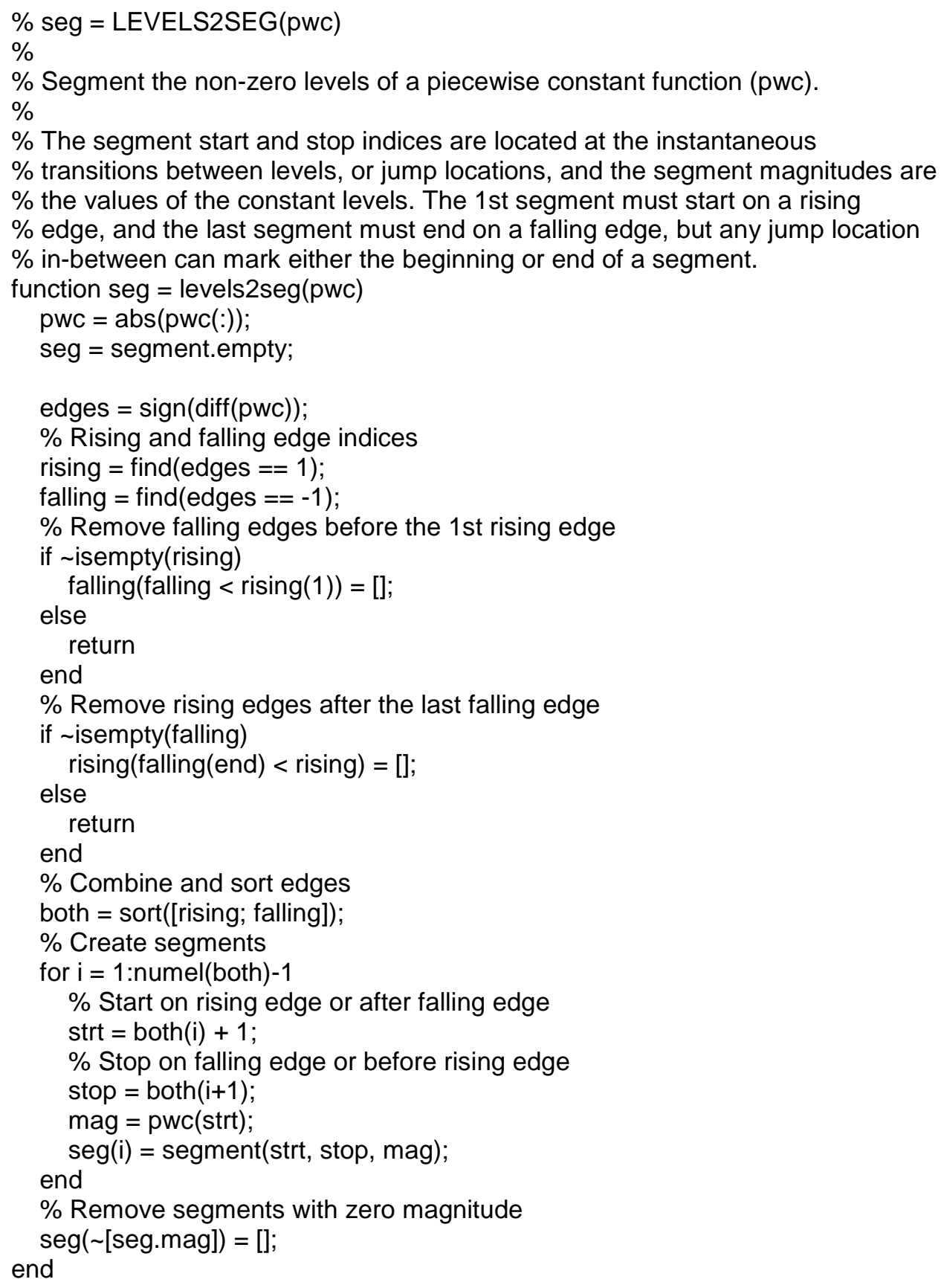




\section{B.3.6 limit_HS.m}

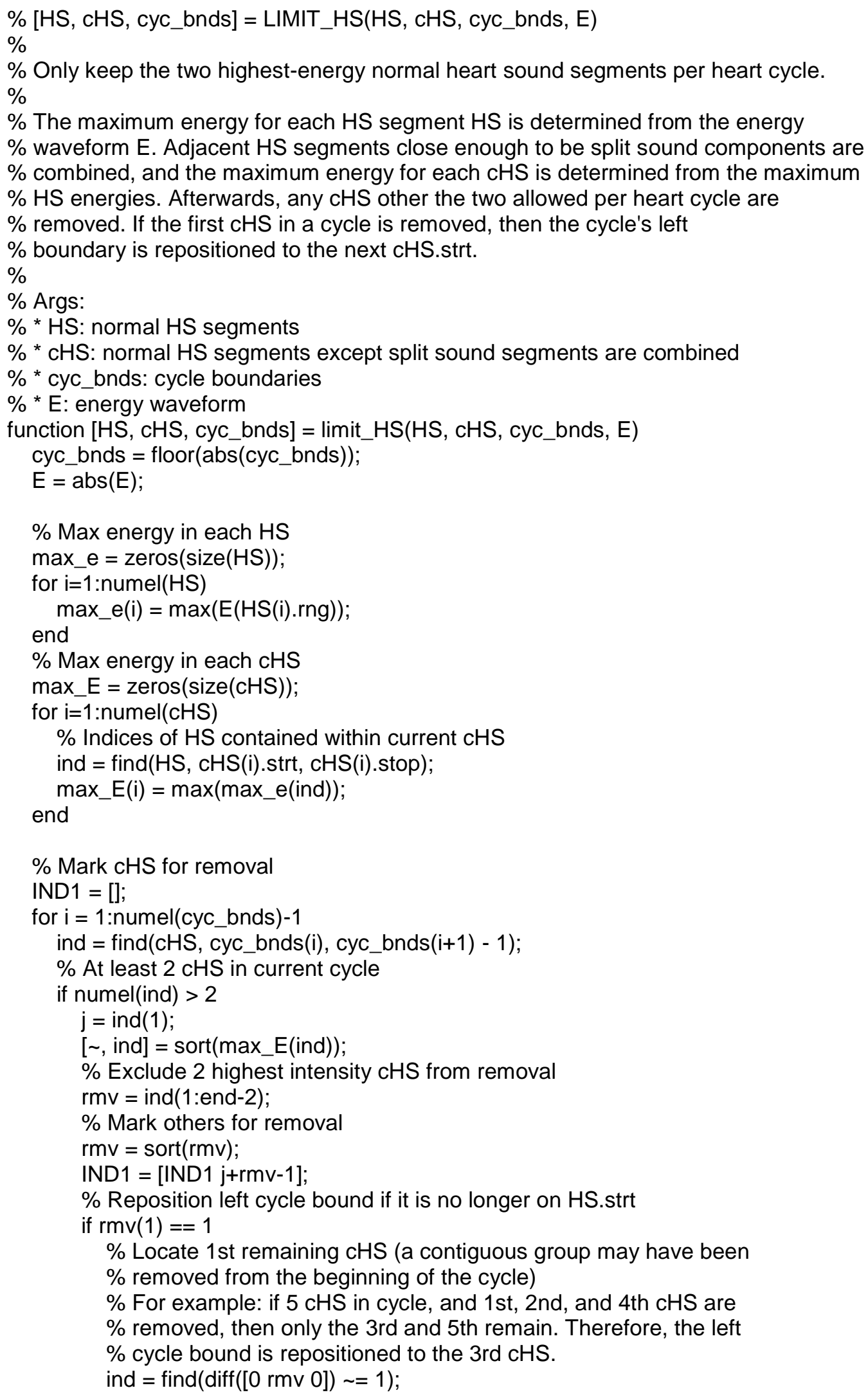




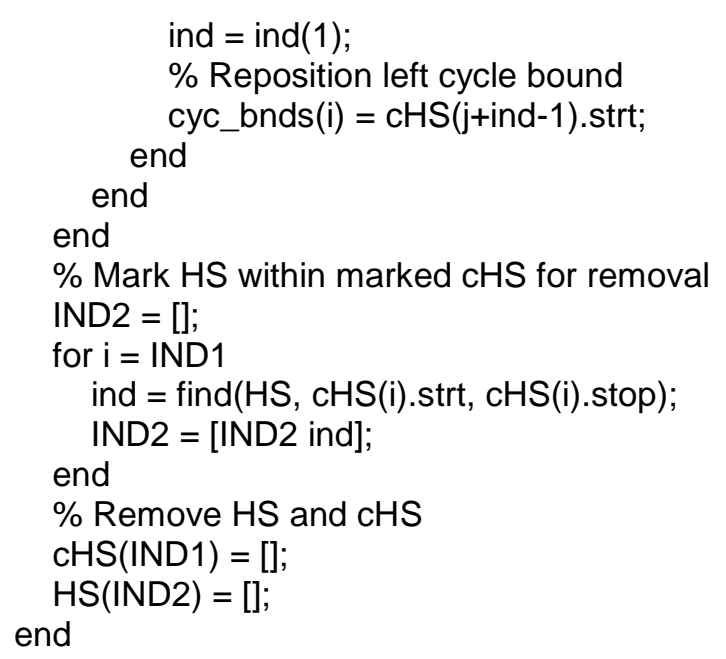

\section{B.3.7 load_PCG.m}

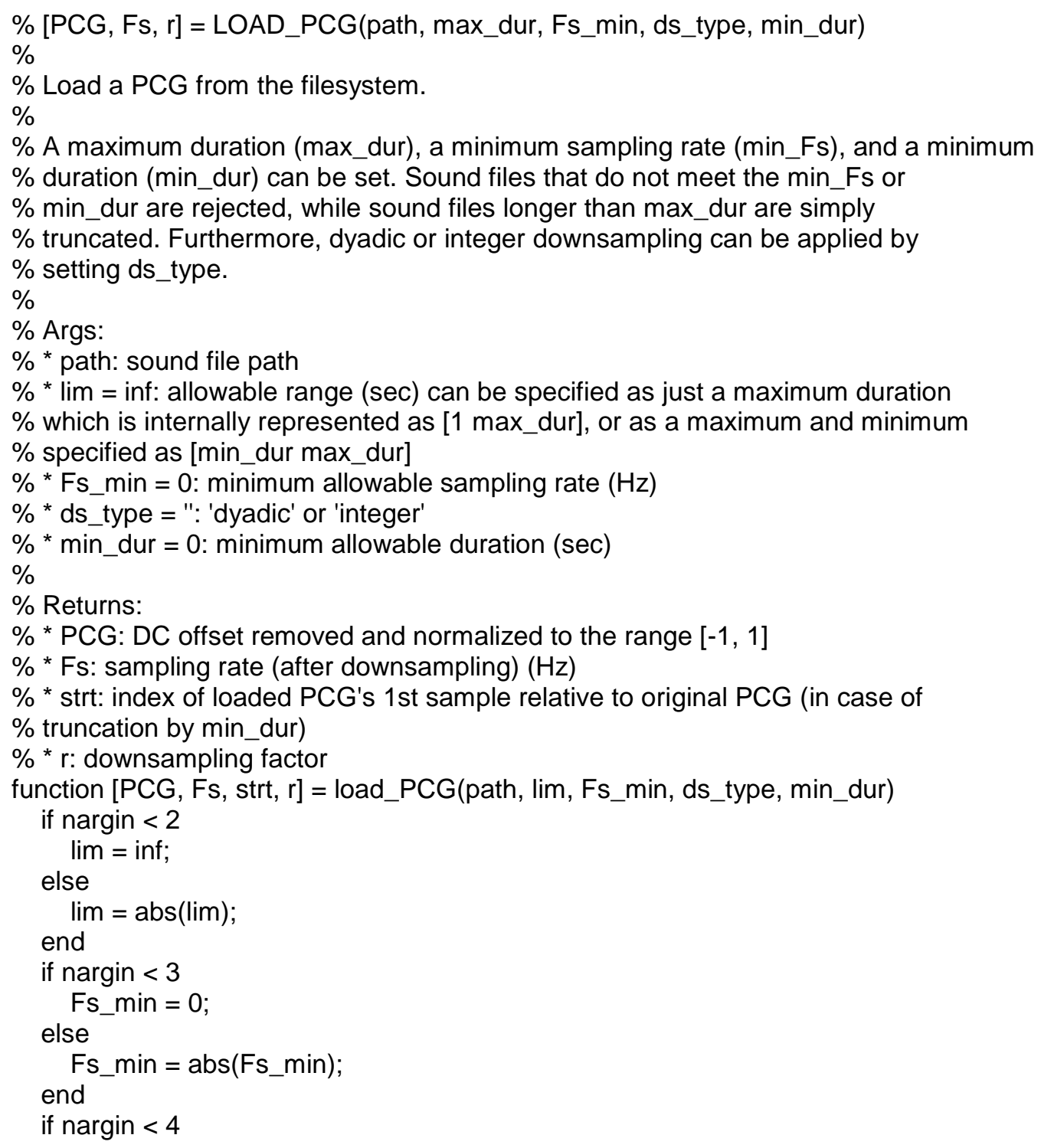




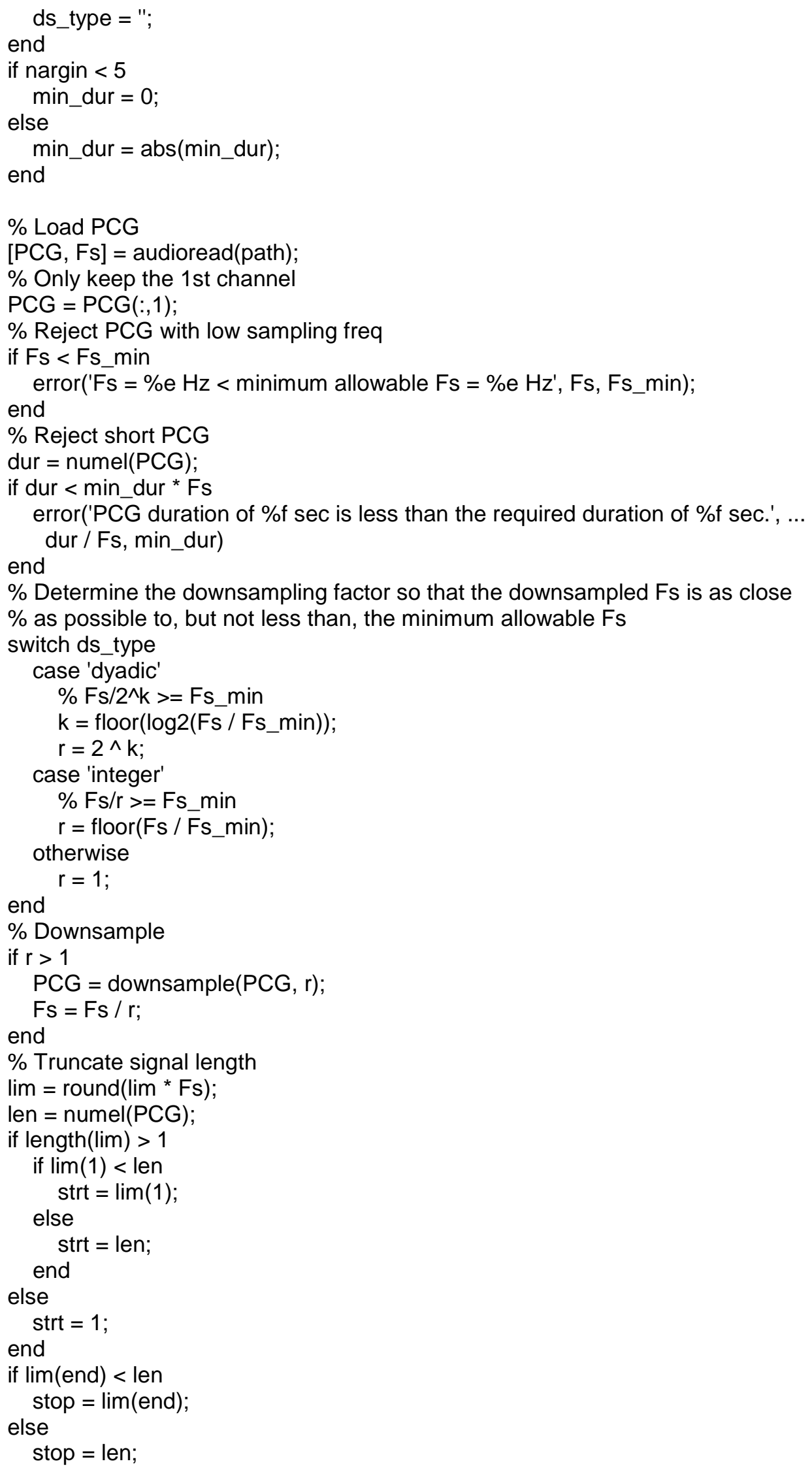




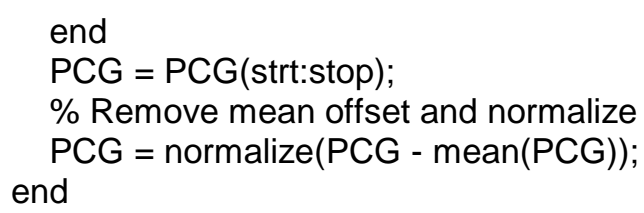

\section{B.3.8 peak_peel.m}

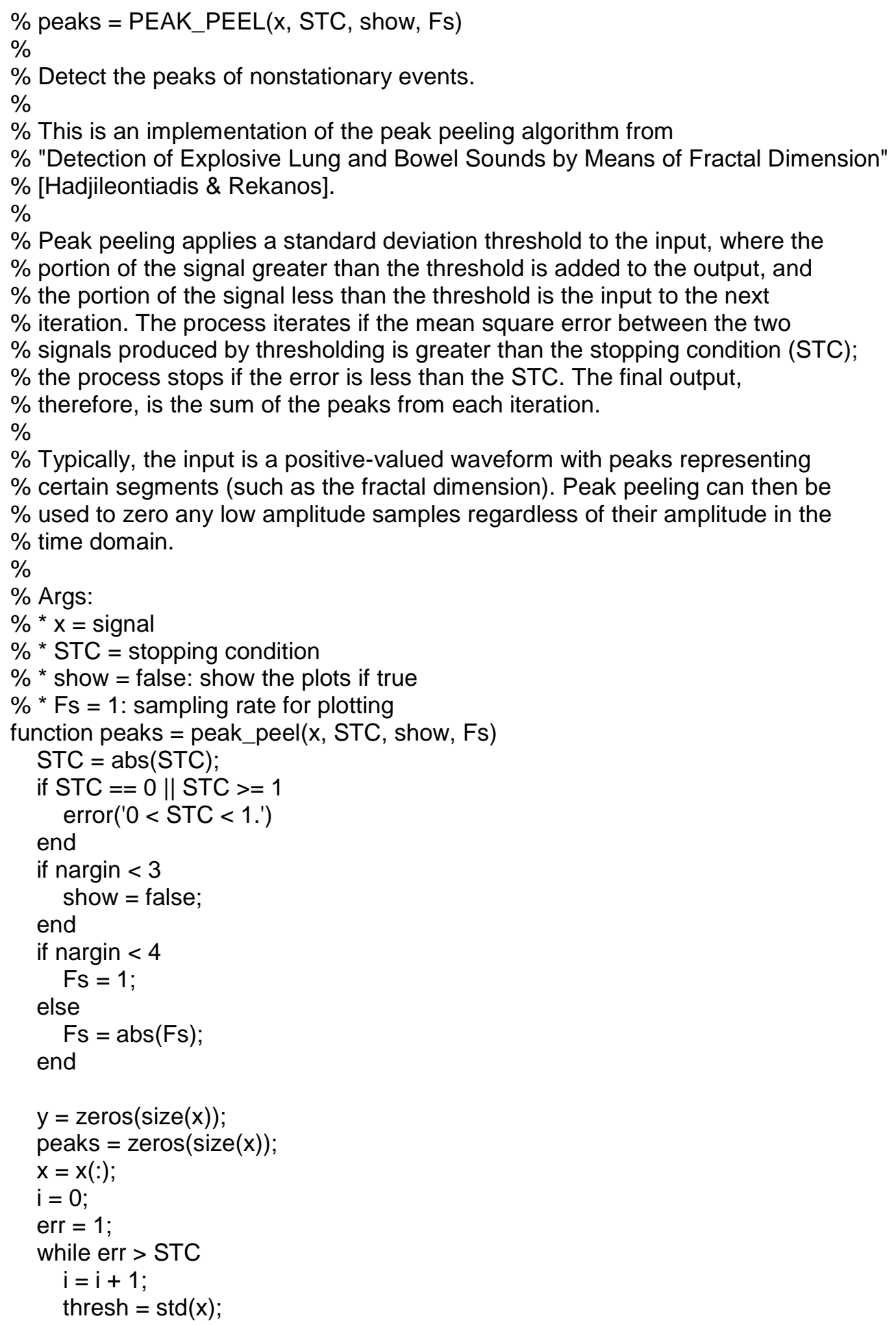




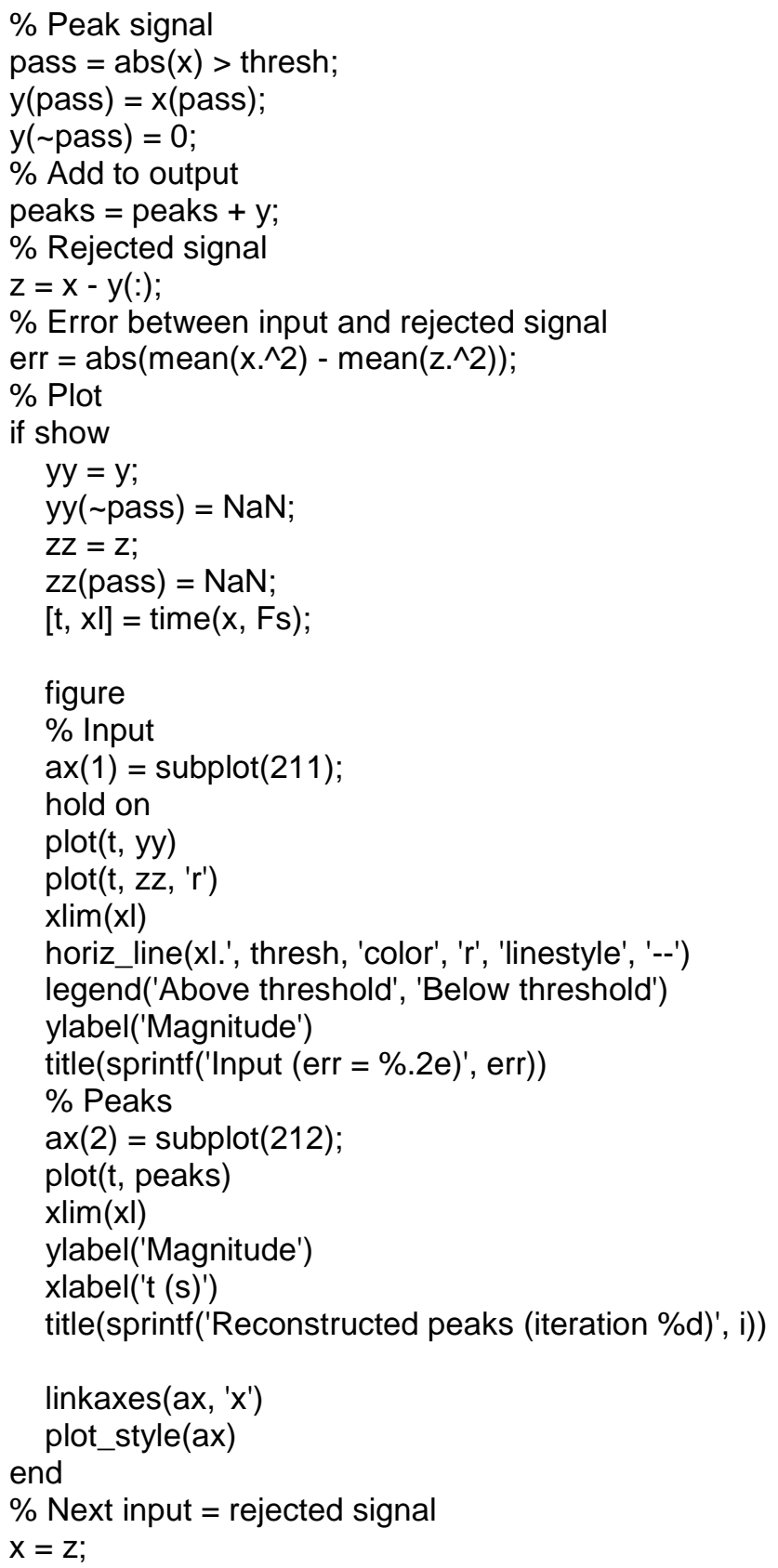

\section{B.3.9 split_HS.m}

$\%\left[H S, T R \_L O C, P K \_L O C\right]=S P L I T \_H S(H S$, env, min_dist, min_height) $\%$

$\%$ Separate heart sound segments that contain split sound components.

$\%$

$\%$ The PCG's envelope is used to find the peaks above a certain threshold in each $\%$ segment. Multiple peaks in a segment indicate split sounds, so the segments $\%$ are separated at the deepest trough between the two tallest peaks.

$\%$

$\%$ Args: 


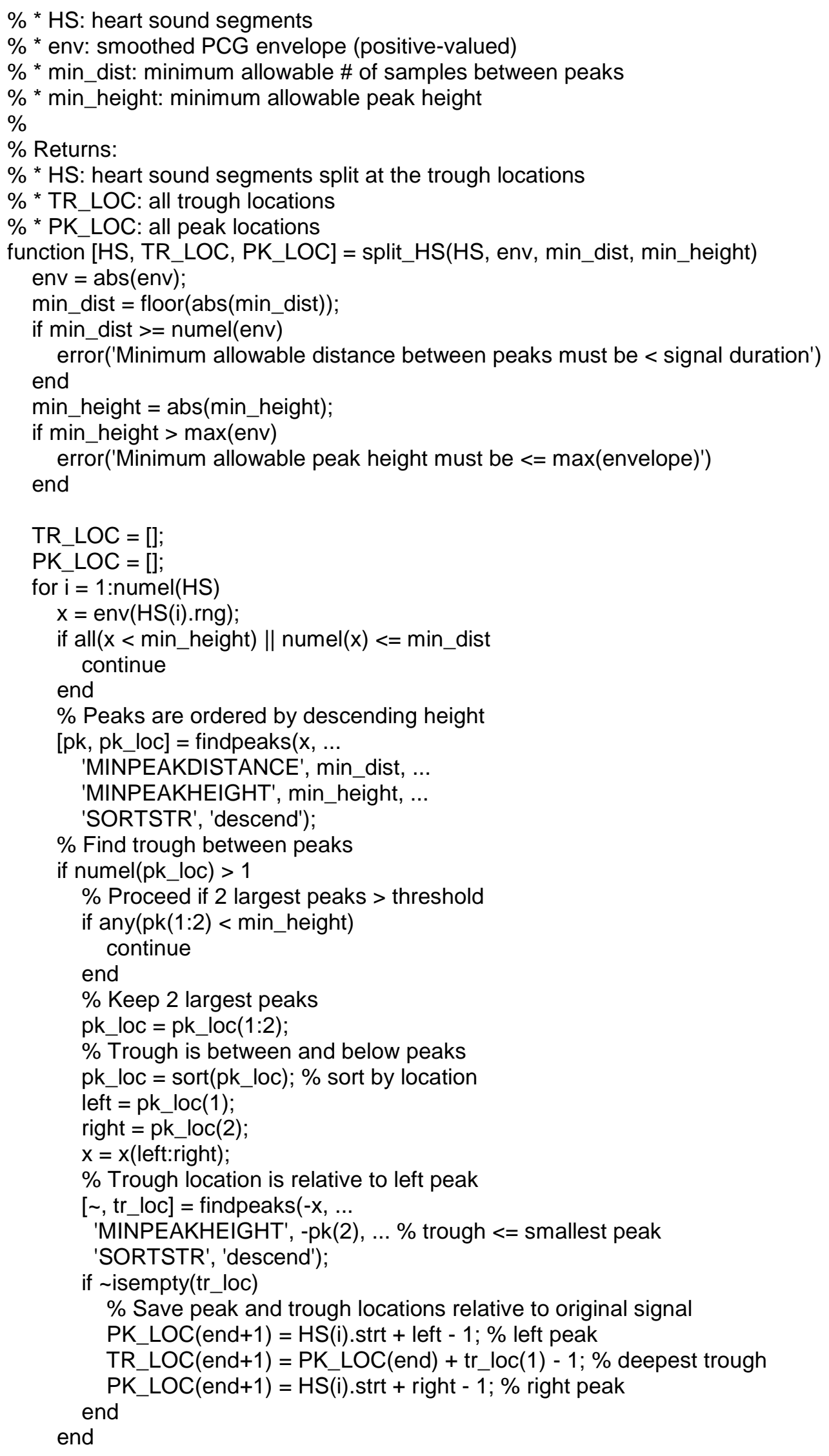


end

\% Split the segments @ the trough locations

HS = split(HS, TR_LOC);

end

\section{B.3.10 st.m}

$\%[$ simpl, D, H] $=\mathrm{ST}(\mathrm{x}, \mathrm{m}, \mathrm{W})$

$\%$

$\%$ Generate a signal that represents time domain complexity.

$\%$

$\%$ This is an implementation of the simplicity transform described in "Accessing

$\%$ Heart Dynamics to Estimate Durations of Heart Sounds" [Nigam \& Priemer].

$\%$

$\%$ The goal is to estimate the system's state space dimension, which is

$\%$ proportional to system complexity. The initial step is the "method of delays"

$\%$ adapted from Taken's embedding theorem, in which a trajectory matrix composed

$\%$ of delay vectors is constructed. A delay vector is a window of length $m$ that

$\%$ contains a contiguous groups of samples from the time domain signal. The delay

$\%$ vector is shifted one sample at a time and stored in the rows of the

$\%$ trajectory matrix until the vector reaches the end of the signal. The

$\%$ trajectory matrix is then cross correlated with itself to form the correlation

$\%$ matrix. Next, the eigenvalues of the correlation matrix, or the singular

$\%$ spectrum, are arranged in descending order. Complex signals tend to have many

$\%$ small-magnitude, eenly distributied eigenvalues, while simple signals tend to

$\%$ have only one or two large-magnitude eigenvalues. This is analogous to the

$\%$ relationship between entropy and the probability mass function (pmf) of a

$\%$ random experiment. Uncertain outcomes tend to have high entropy and evenly

$\%$ distributed pmf's, while certain outcomes tend to have low entropy and skewed

$\%$ pmf's. Since the entropy is an estimate of the number of bits required to

$\%$ represent an outcome, the entropy is likewise an estimate of the number of

$\%$ dimensions required to represent the system in state space. Therefore, the

$\%$ entropy of the singular spectrum is proportional to signal complexity, and

$\%$ simplicity is just the inverse of complexity.

$\%$

$\%$ By default, the output is a scalar because the window length $W$ is the same as

$\%$ the signal length $\mathrm{L}$. If $\mathrm{W}<\mathrm{L}$, then the output has the same dimensions as the

$\%$ input.

$\%$

$\%$ Args:

$\%{ }^{*} \mathrm{~m}=$ delay vector length

$\%{ }^{*} \mathrm{~W}=$ window length

$\%$

$\%$ Returns:

$\%$ * simpl: simplicity

$\%$ * D: each column is the singular spectrum for a window (eigenvalues are

$\%$ arranged in descending order)

$\%{ }^{*} \mathrm{H}$ : entropy

function [simpl, D, H] $=\mathrm{st}(\mathrm{x}, \mathrm{m}, \mathrm{W})$

$\mathrm{L}=\operatorname{numel}(\mathrm{x})$;

if nargin $<3$

$\mathrm{W}=\mathrm{L}$;

end

if $\mathrm{W}==\mathrm{L}$

simpl $=0$;

offset $=1$; 


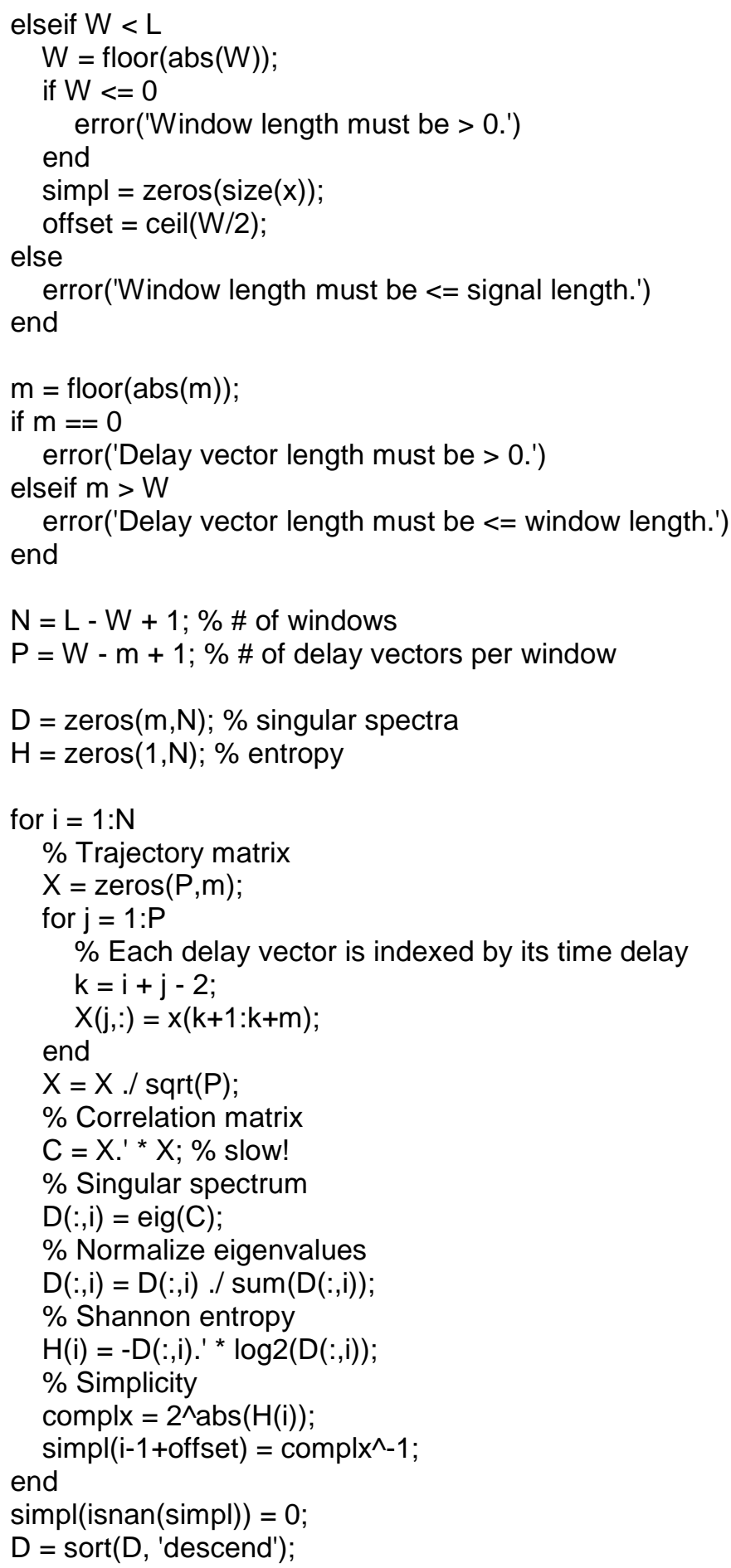

\section{B.4 Miscellaneous}

\section{B.4.1 closest.m}

$\% \mathrm{i}=$ CLOSEST(vals, val)

$\%$ 
$\%$ Locate the index $\mathrm{i}$ of the element from the array vals closest in value to the $\%$ desired value val.

function [i, cval] $=$ closest $($ vals, val $)$

diff $=$ abs(vals(:) - val);

end

$[\sim, \mathrm{i}]=\min ($ diff);

\section{B.4.2 energy.m}

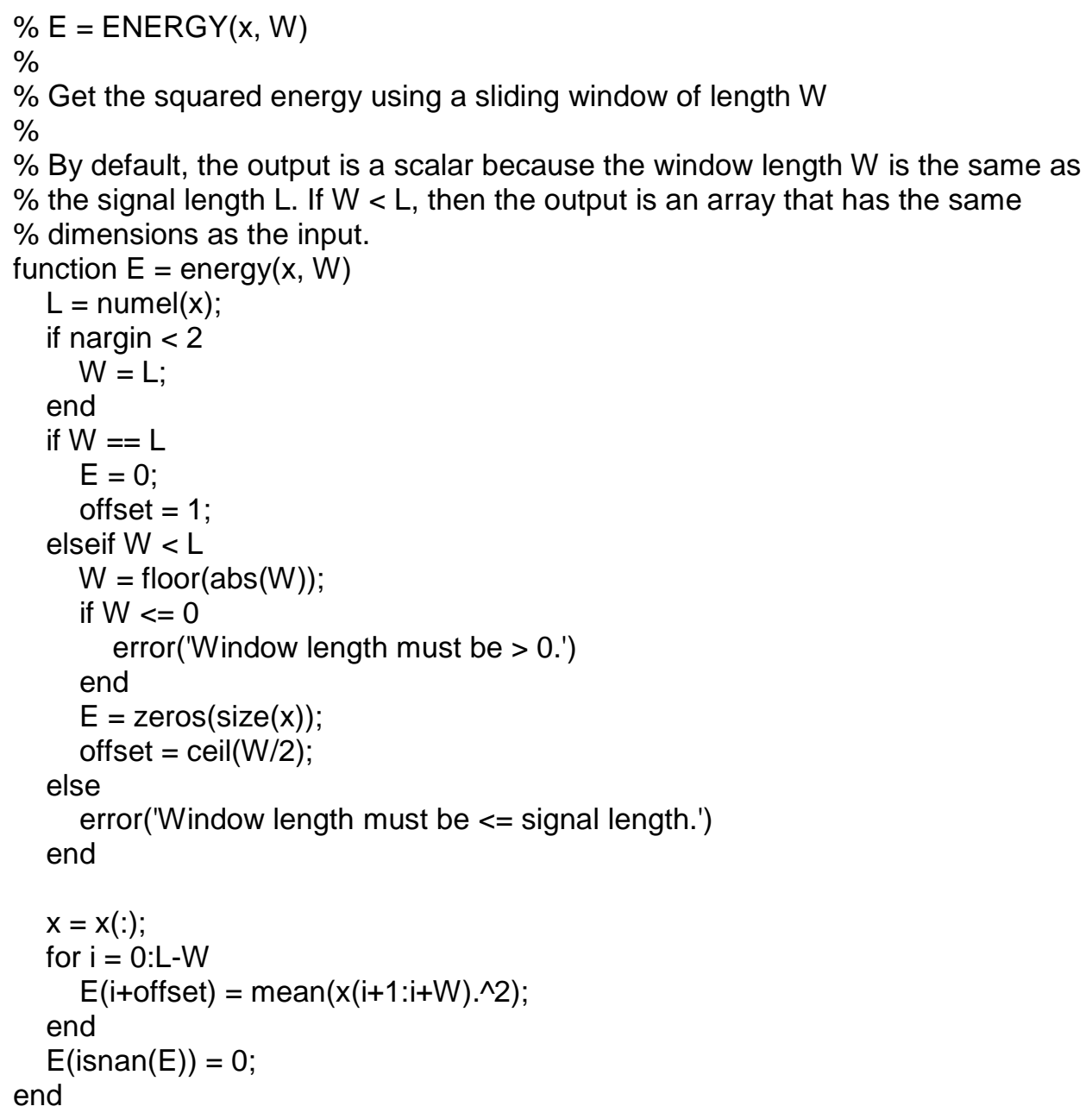

\section{B.4.3 env.m}

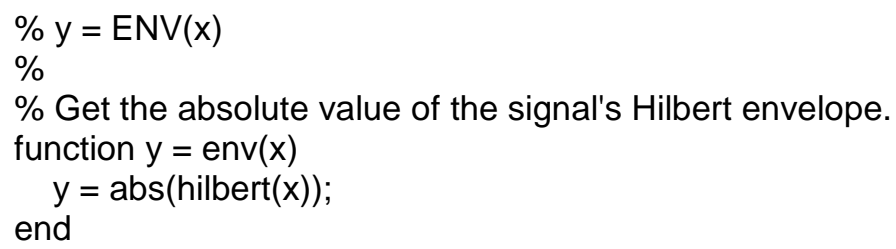

\section{B.4.4 nfft.m}

$\%[Y, f]=\operatorname{NFFT}(x)$

$\%$ 


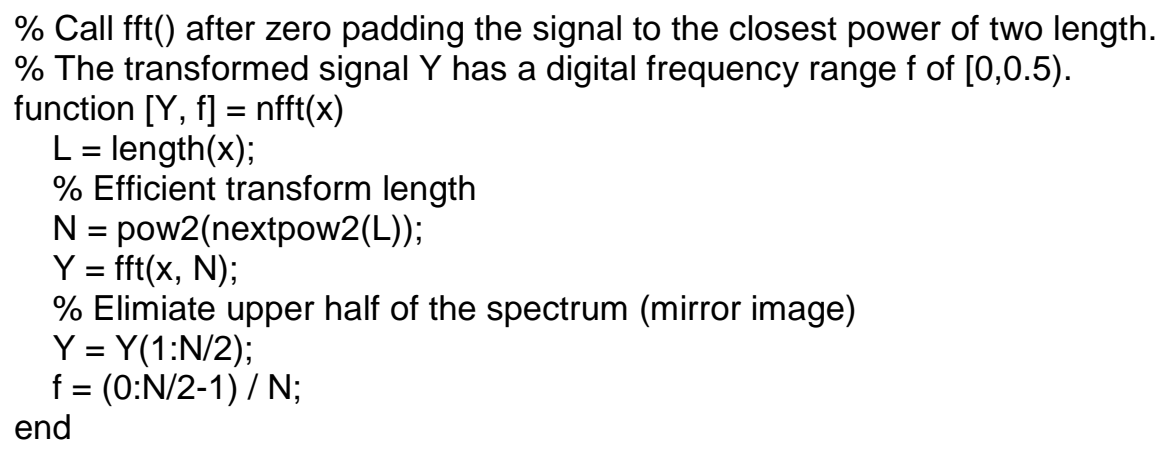

\section{B.4.5 normalize.m}

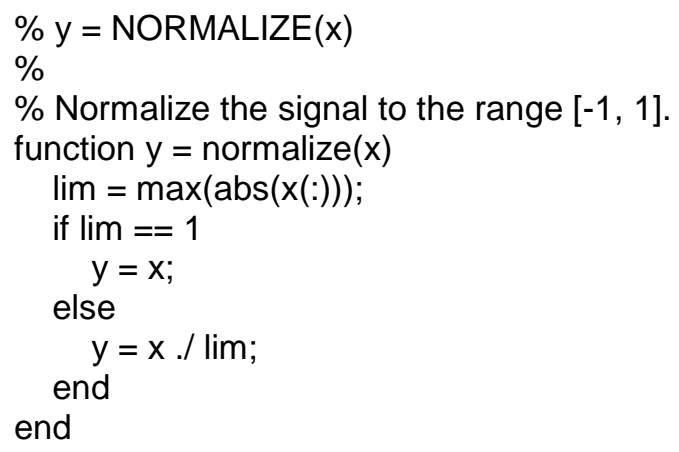

\section{B.4.6 pcg_descr.m}

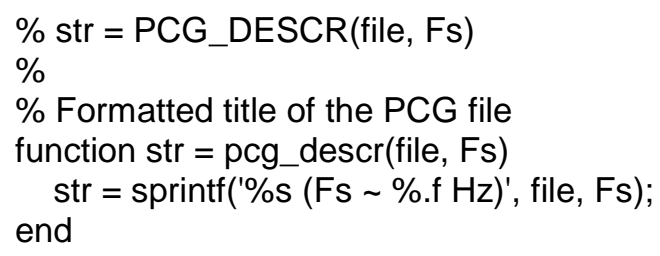

\section{B.4.7 rect.m}

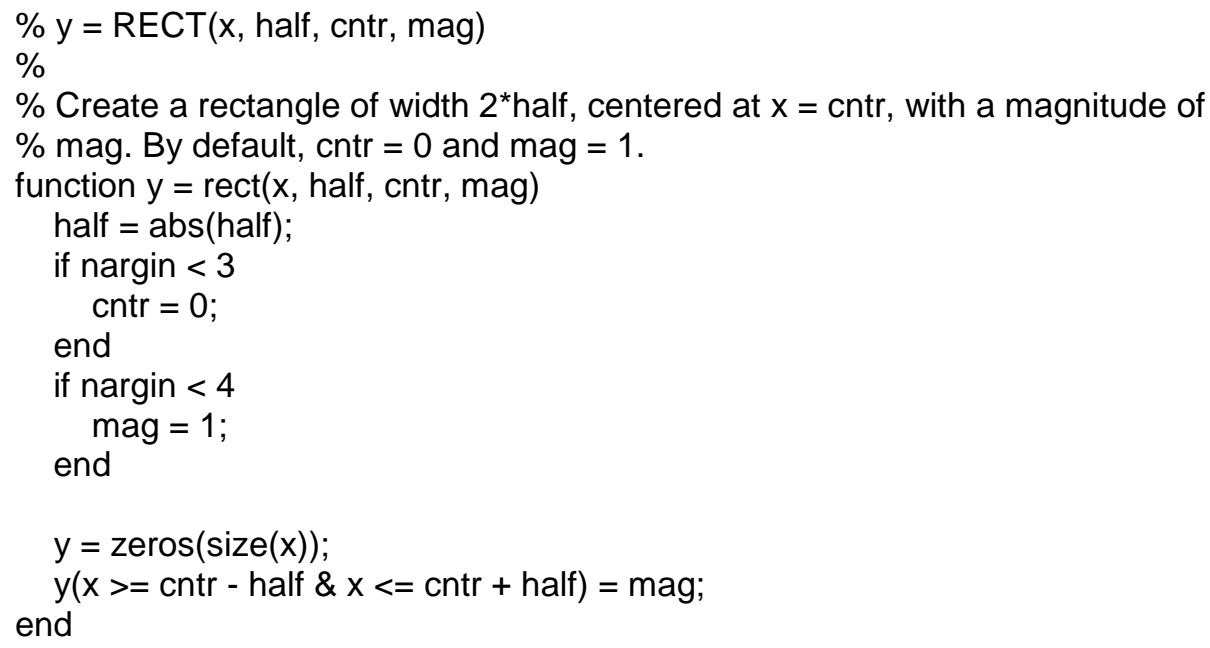

\section{B.4.8 shannon_energy.m}




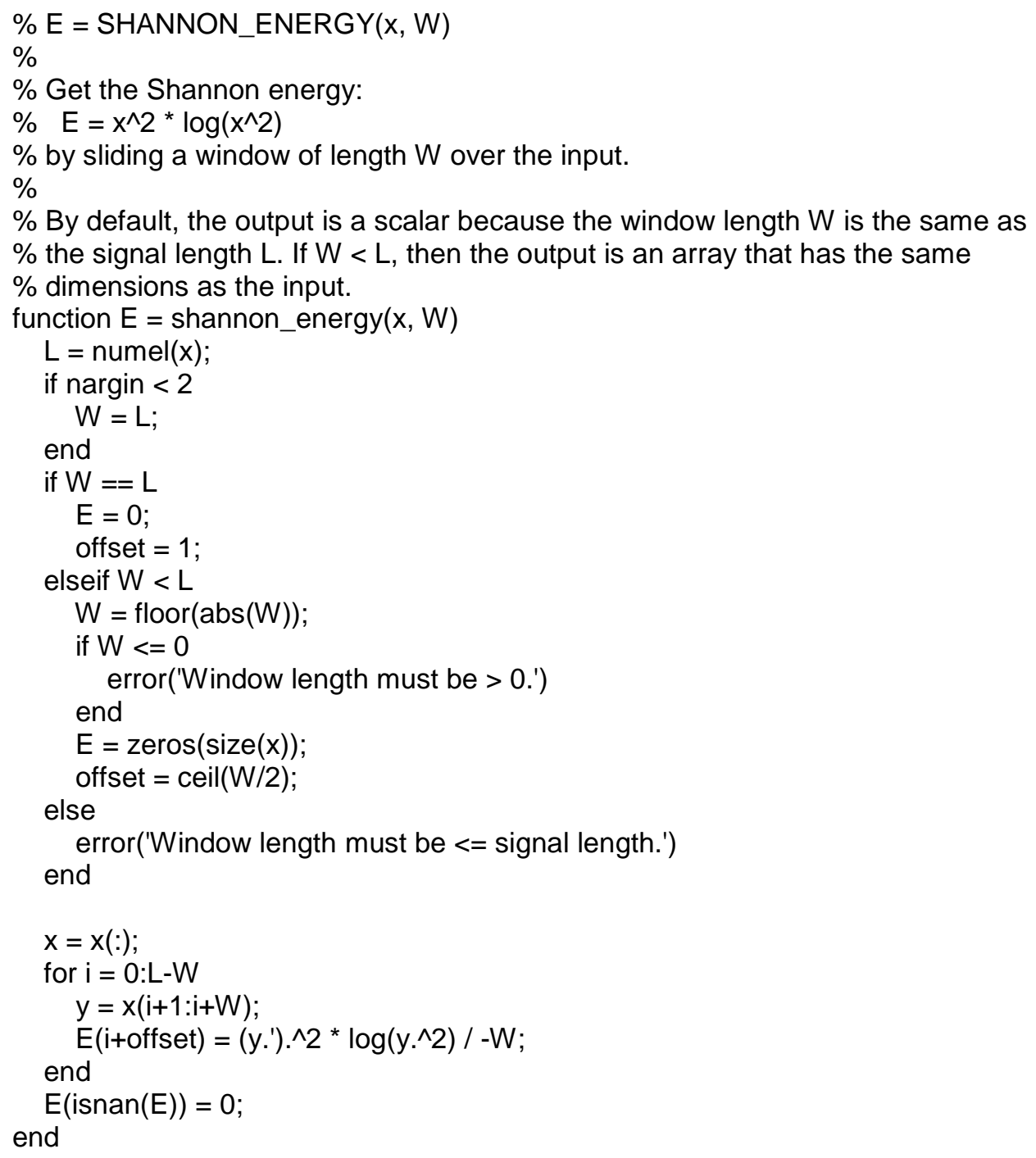

\section{B.4.9 smooth.m}

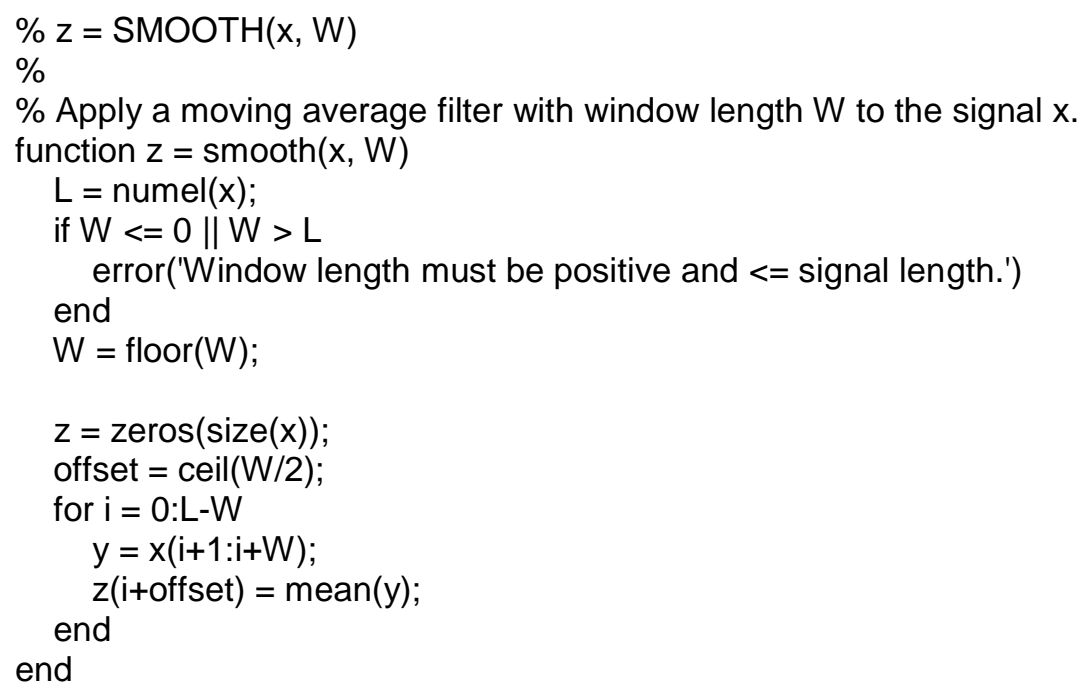




\section{B.4.10 time.m}

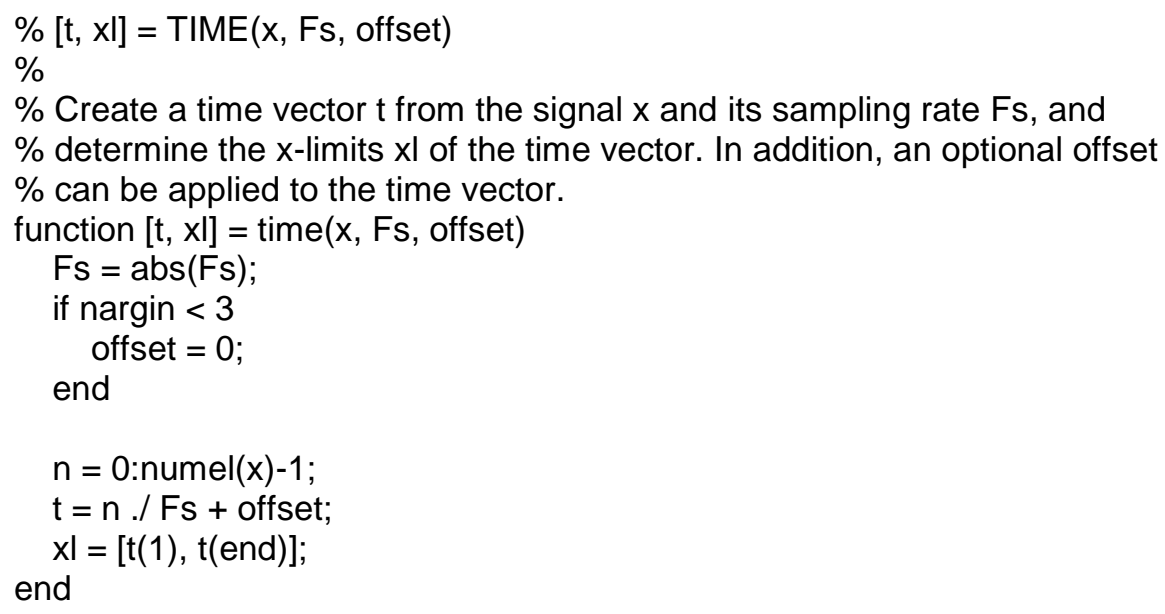

\section{B.5 Plotting}

\section{B.5.1 fmt_line_arg.m}

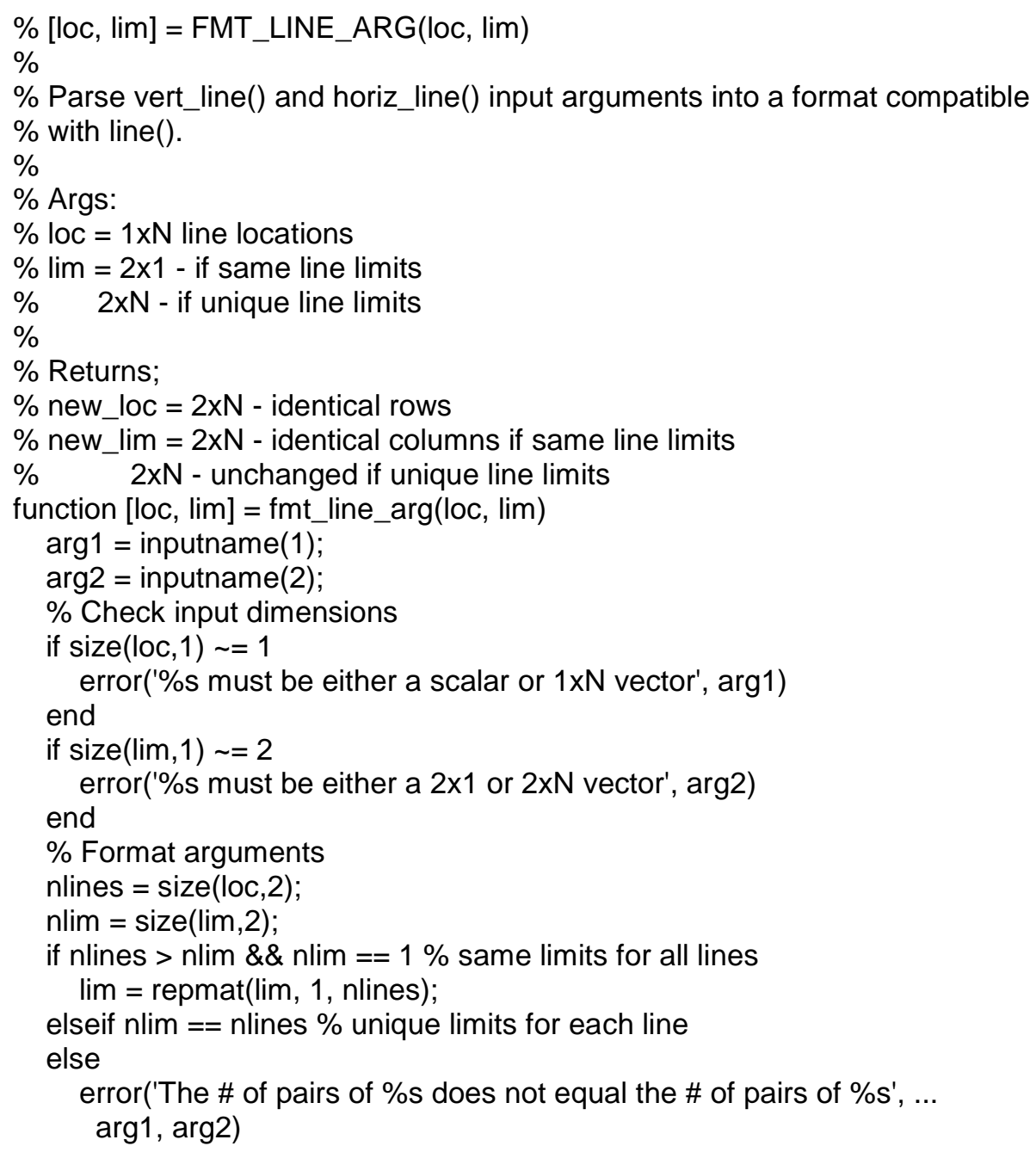




$$
\begin{aligned}
& \text { end } \\
& \text { loc = [loc; loc }] \text {; } \\
& \text { end }
\end{aligned}
$$

\section{B.5.2 horiz_line.m}

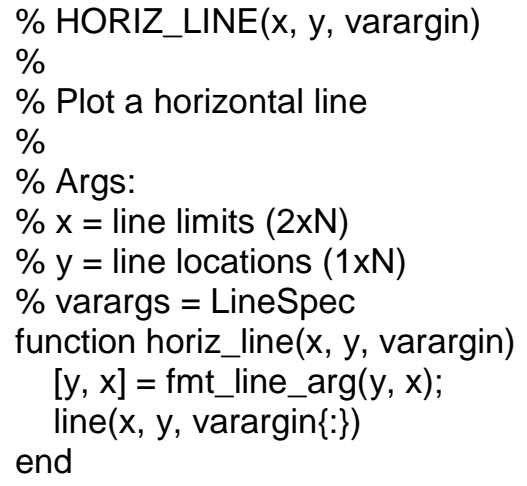

\section{B.5.3 plot_style.m}

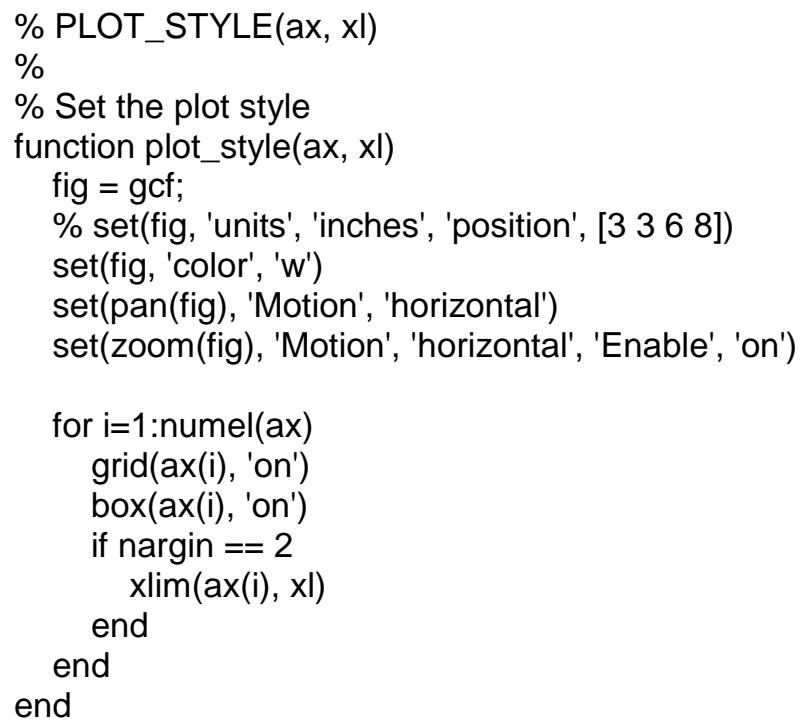

\section{B.5.4 vert_line.m}

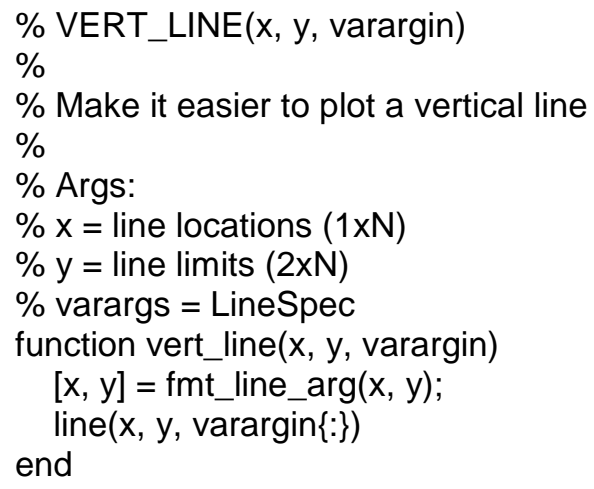




\section{Class Definitions and Methods}

\section{C.1@segment}

\section{C.1.1 combine.m}

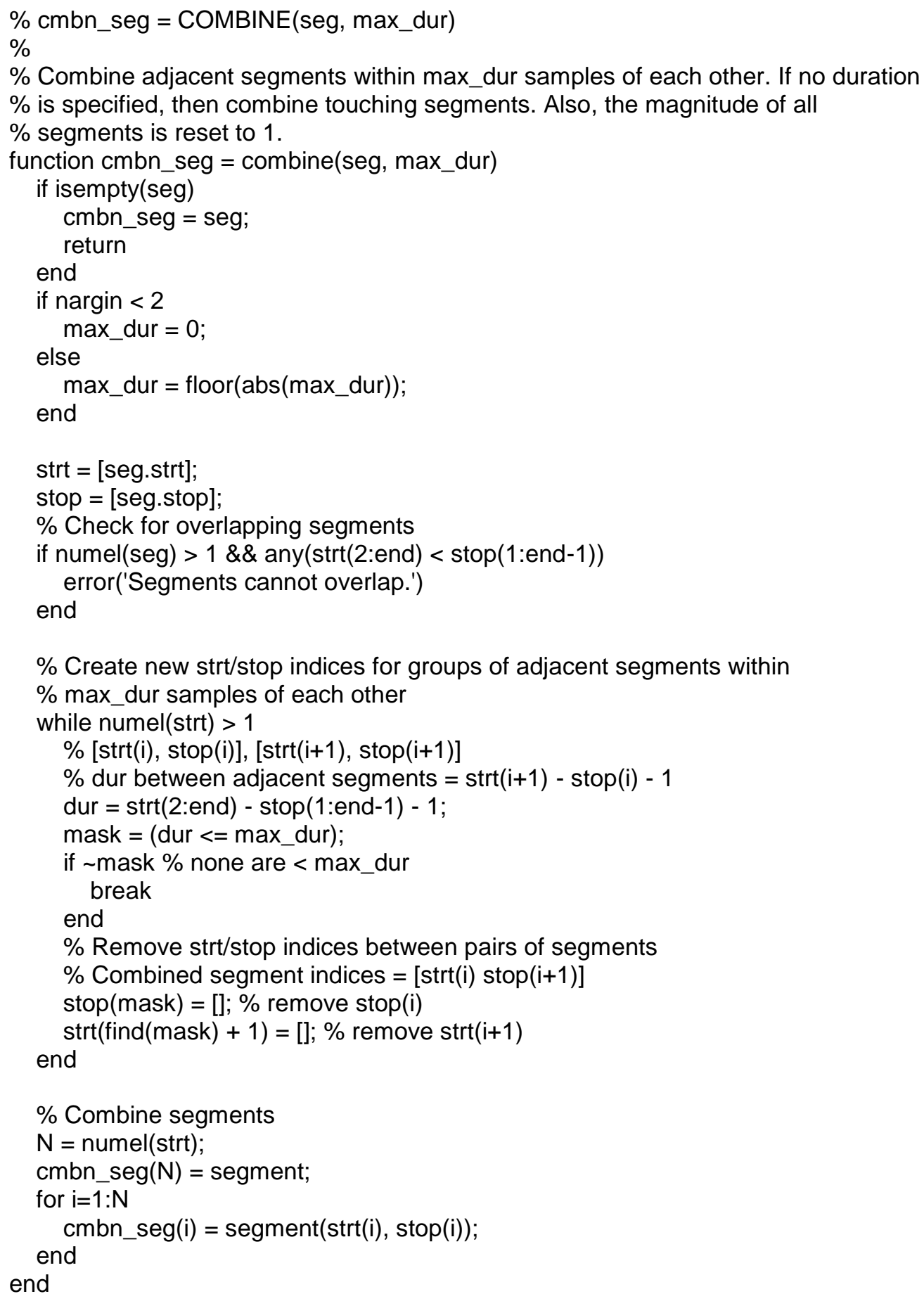

\section{C.1.2 find.m}




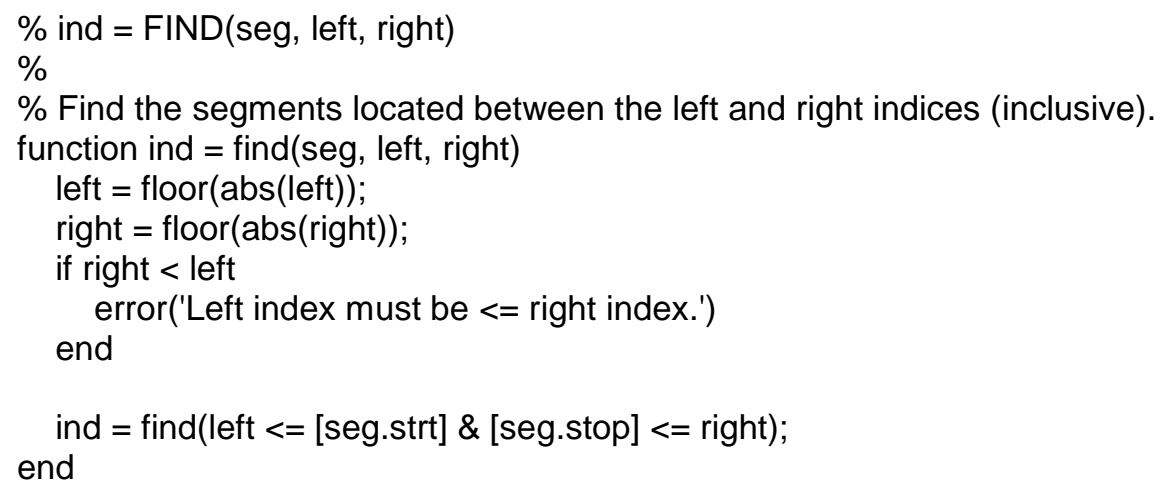

\title{
C.1.3 levels.m
}

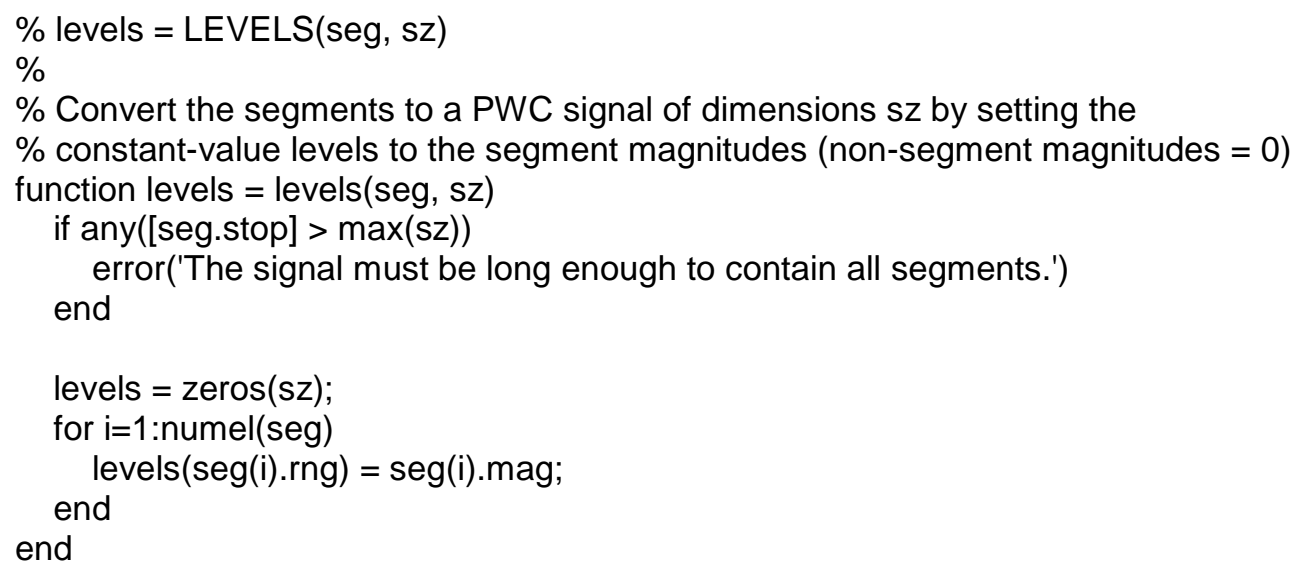

\section{C.1.4 mask.m}

$\%$ function mask = MASK(seg, sz, lvl)

$\%$

$\%$ Convert the segments to a binary signal of dimensions sz, where the "on"

$\%$ magnitude is specified by lvl (default $=1)$.

function mask $=$ mask (seg, sz, lvl)

if any ([seg.stop] $>\max (\mathrm{sz}))$ error('The signal must be long enough to contain all segments.') end

if nargin $<3$

$$
\mid \mathrm{vl}=1
$$

end

mask $=$ zeros(sz);

for $\mathrm{i}=1$ :numel(seg)

$\operatorname{mask}(\operatorname{seg}(\mathrm{i}) \cdot \mathrm{rng})=\mid \mathrm{vl}$;

$$
\text { end }
$$

end

\section{C.1.5 segment.m}

\author{
classdef segment \\ properties \\ mag
}




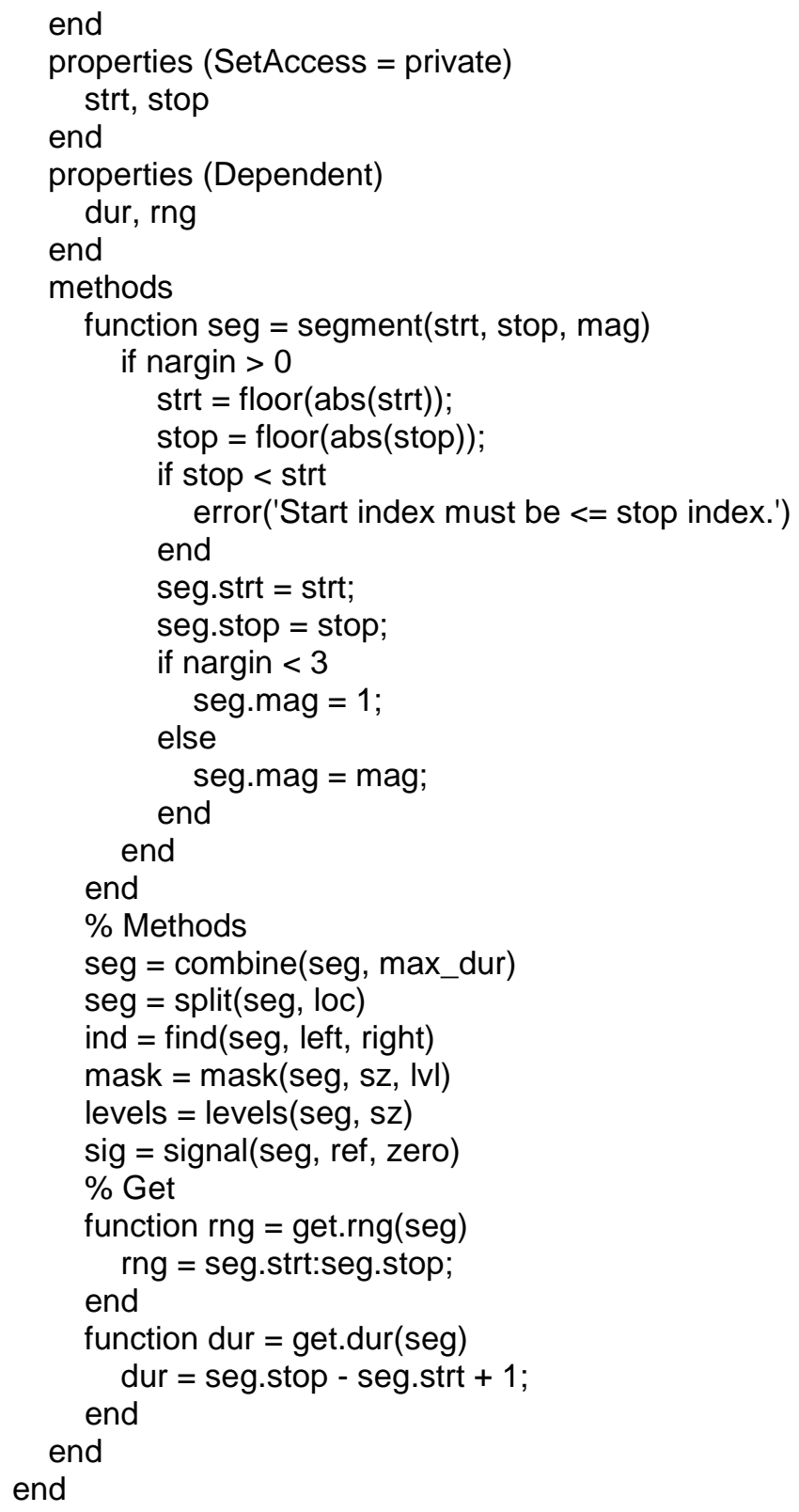

\section{C.1.6 signal.m}

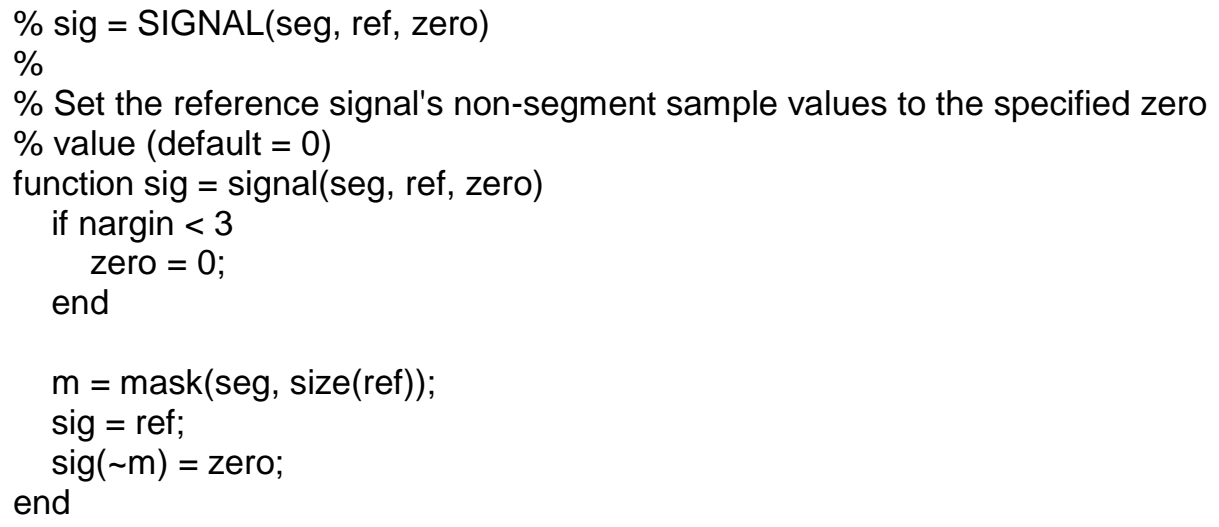




\section{C.1.7 split.m}

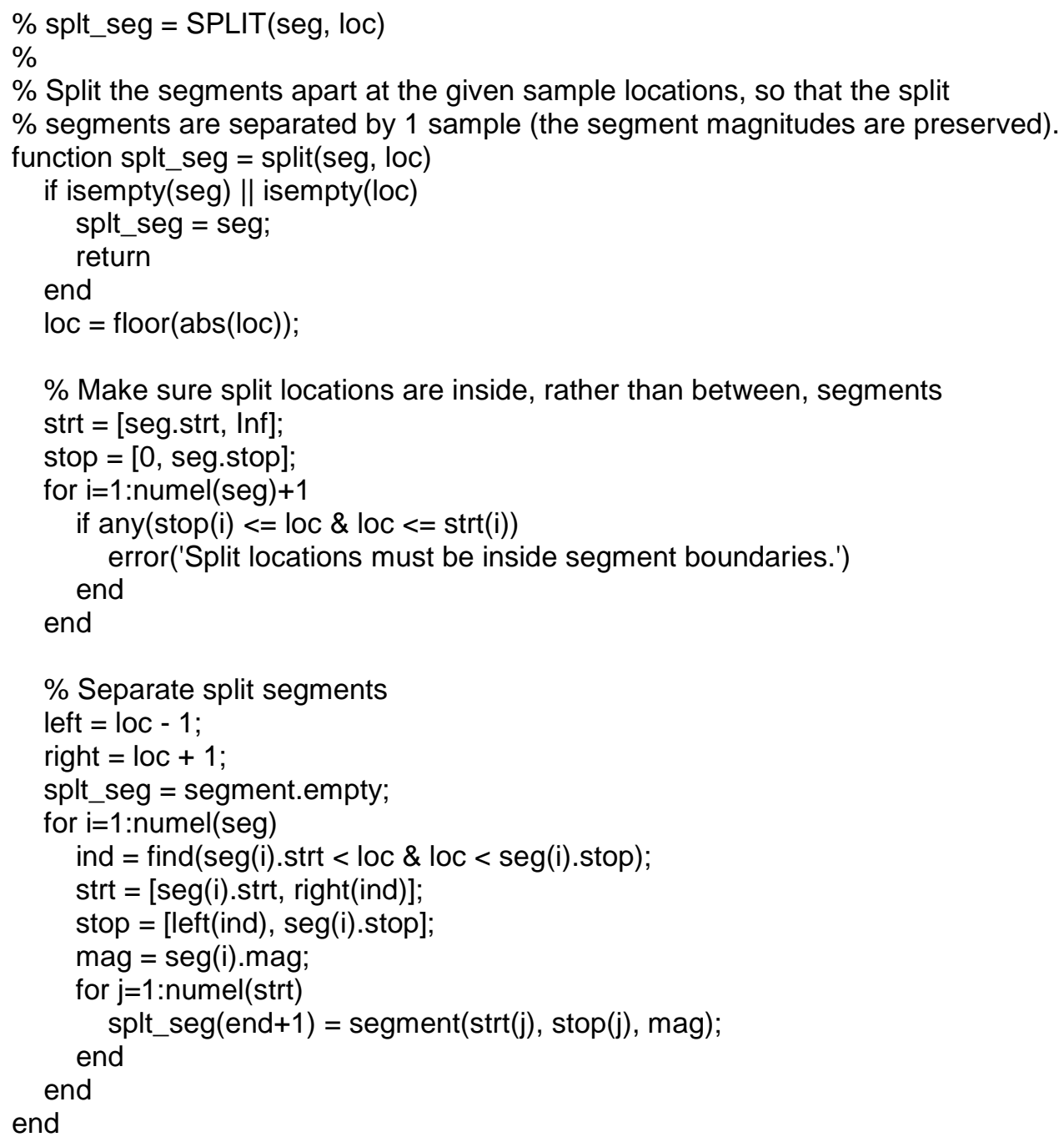

\section{C.2@stethoscope}

\section{C.2.1 cmp_PCG.m}

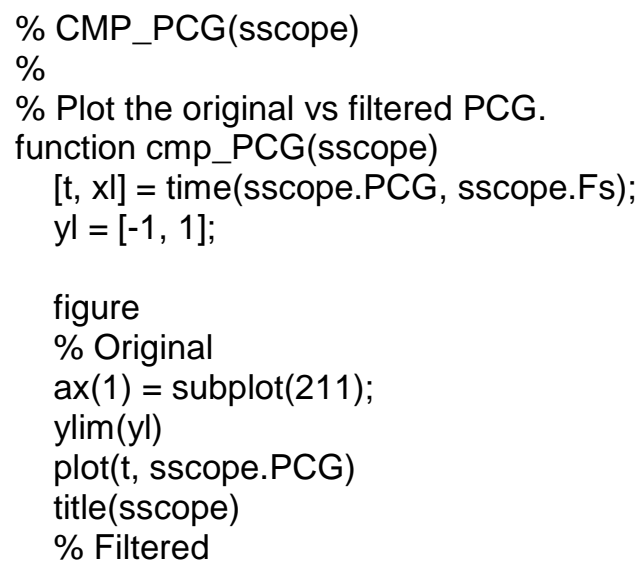




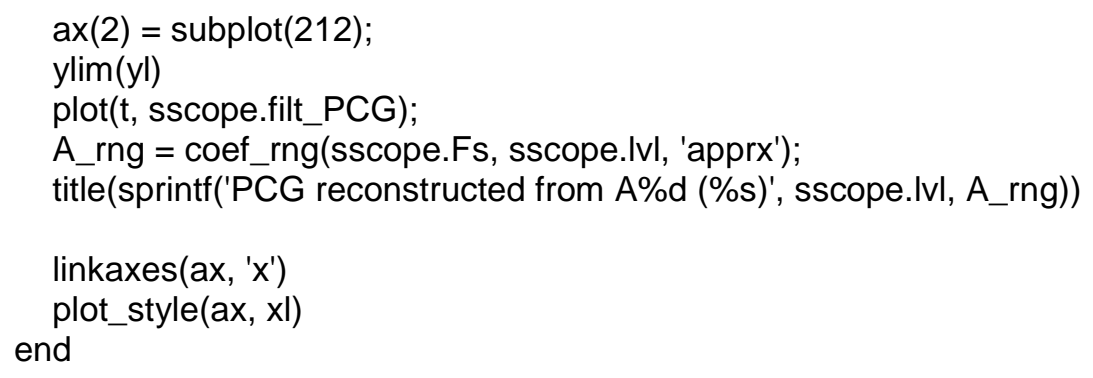

\section{C.2.2 dwt_filt.m}

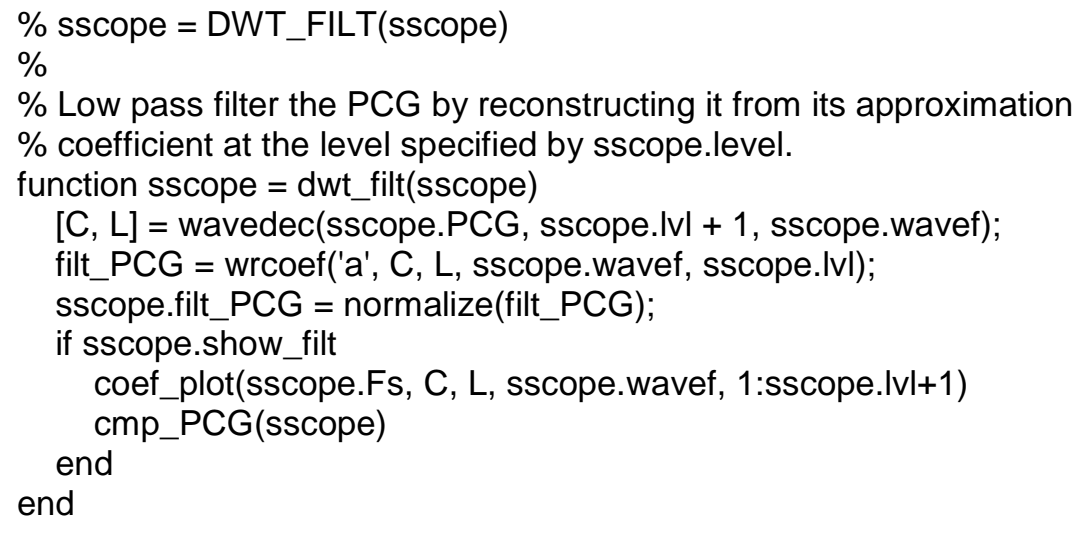

\section{C.2.3 dwt_segment.m}

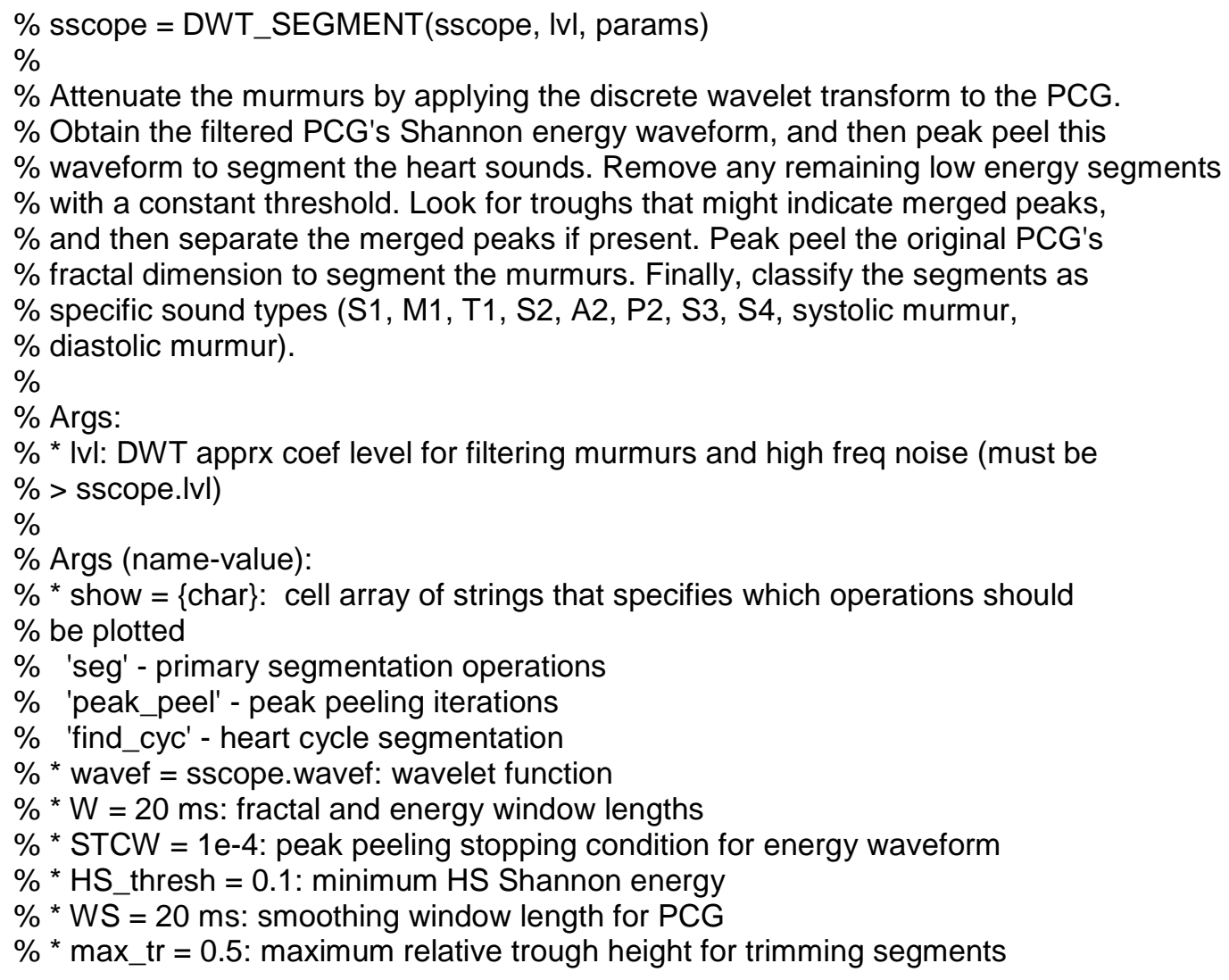




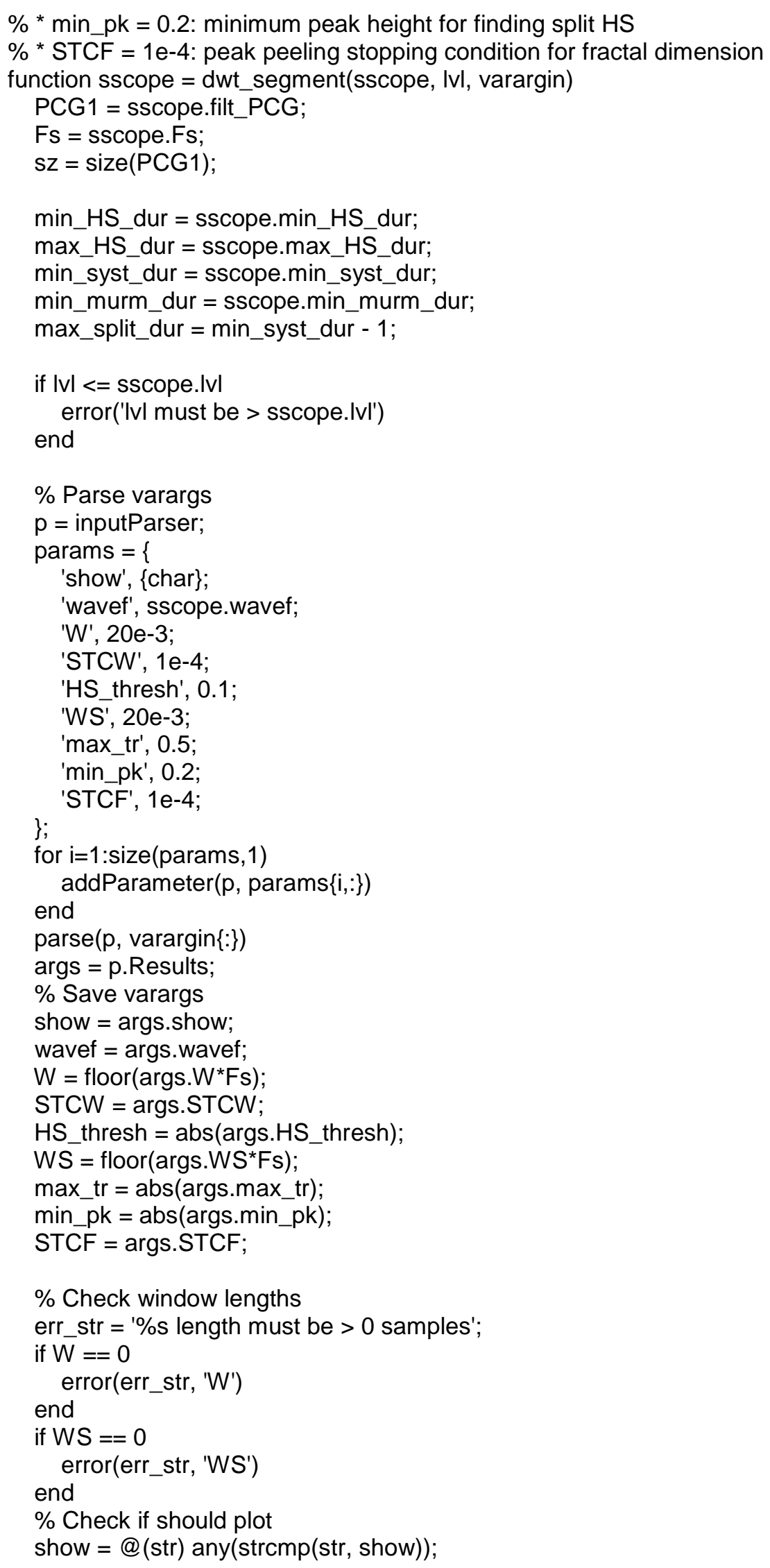




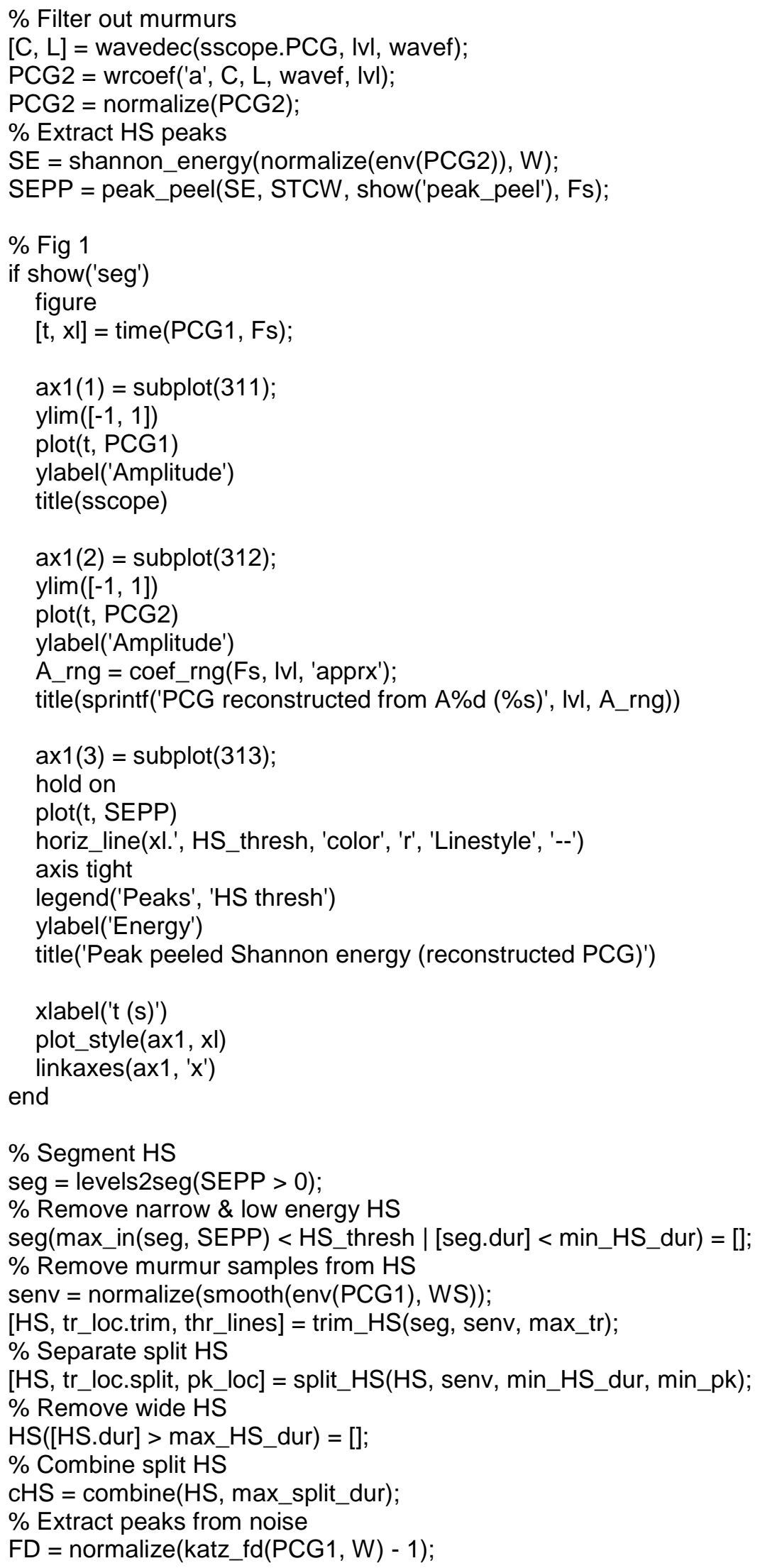




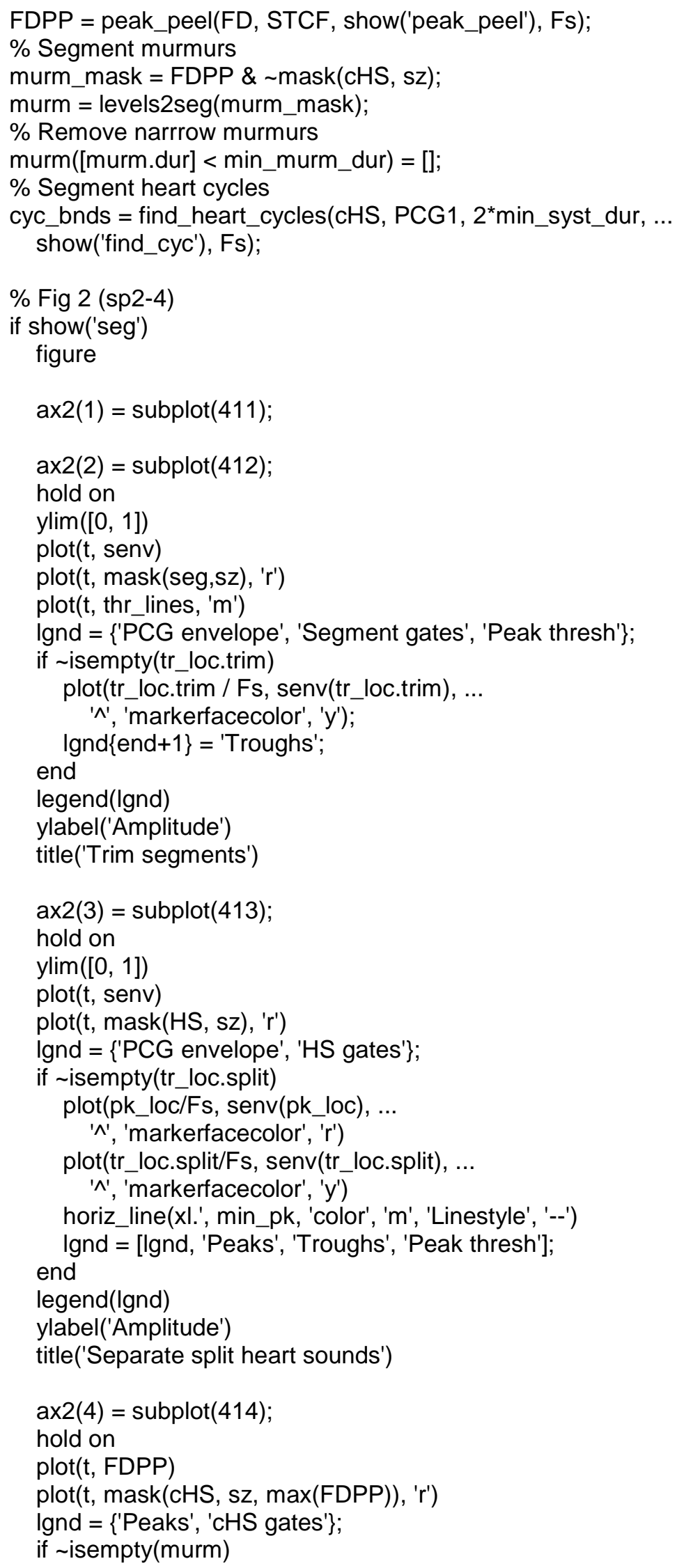




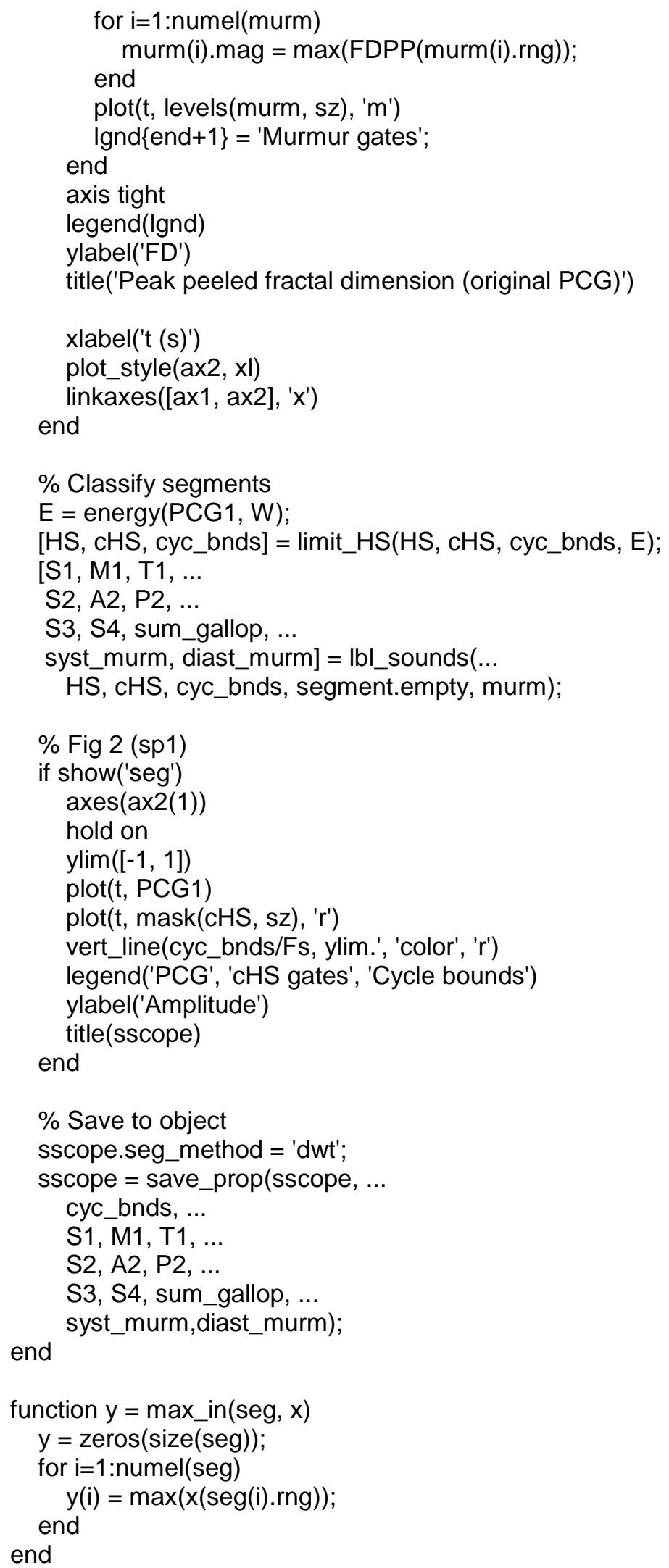




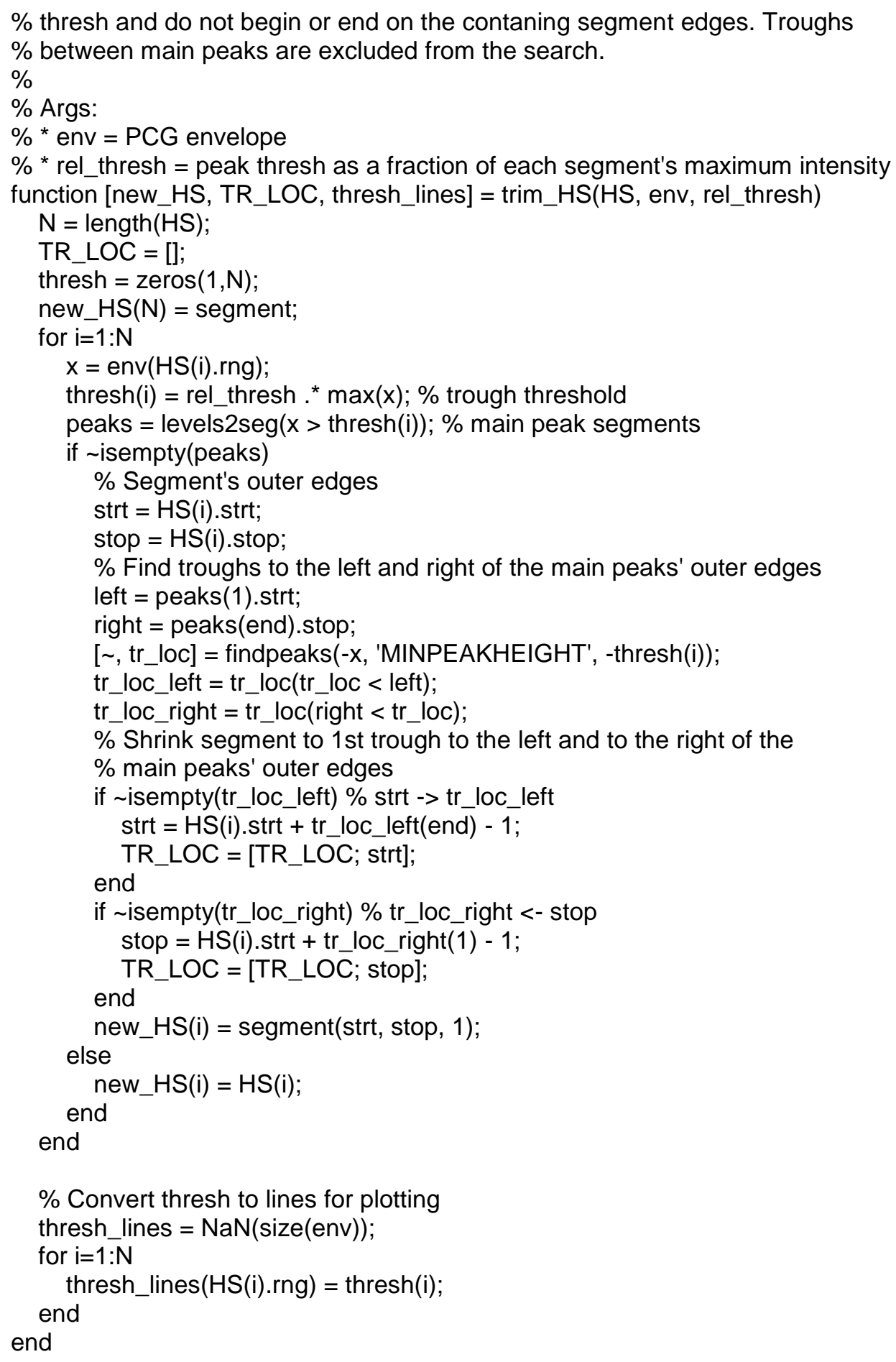

\section{C.2.4 plot.m}

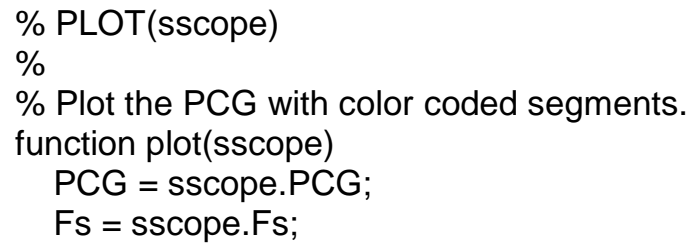




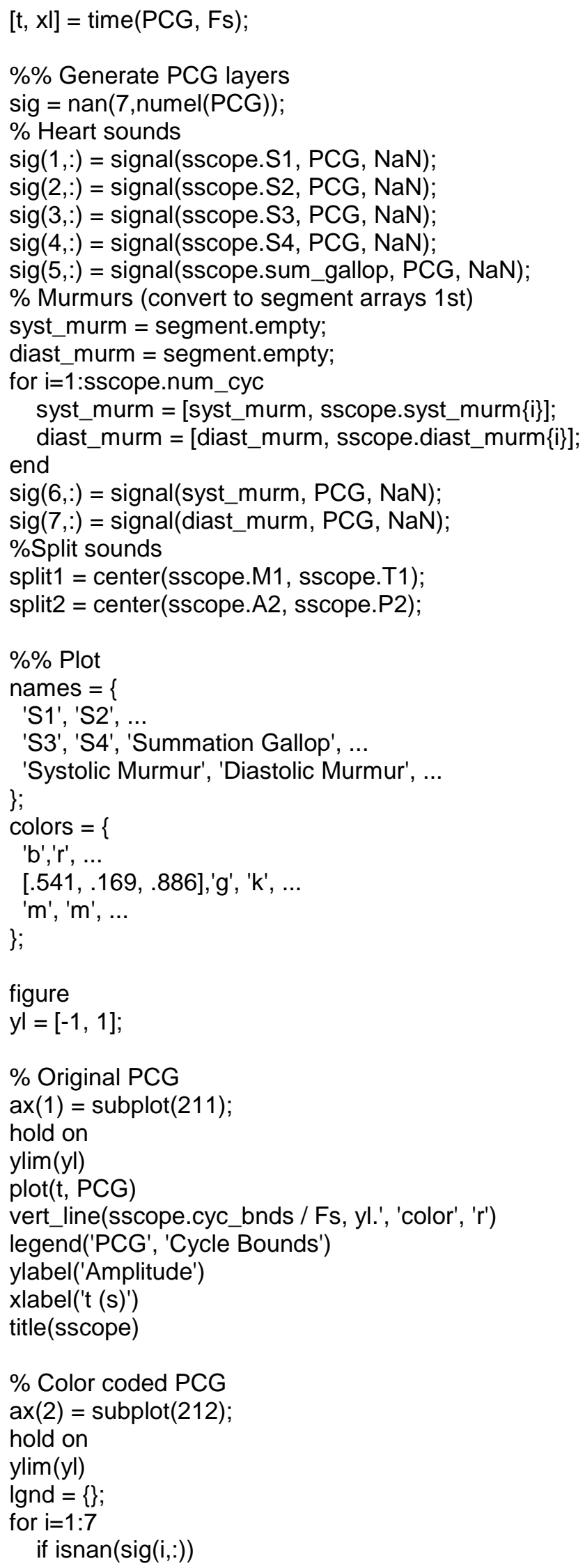




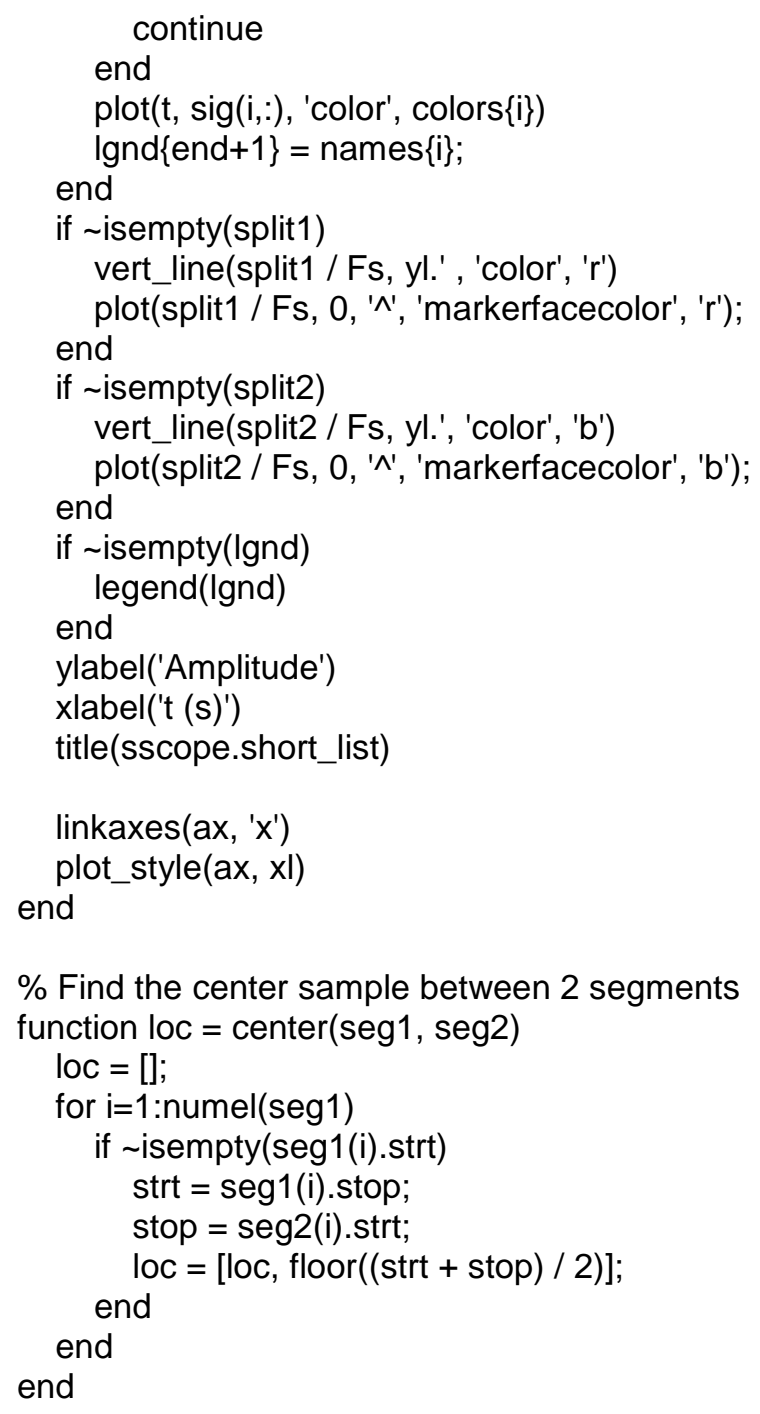

\section{C.2.5 print.m}

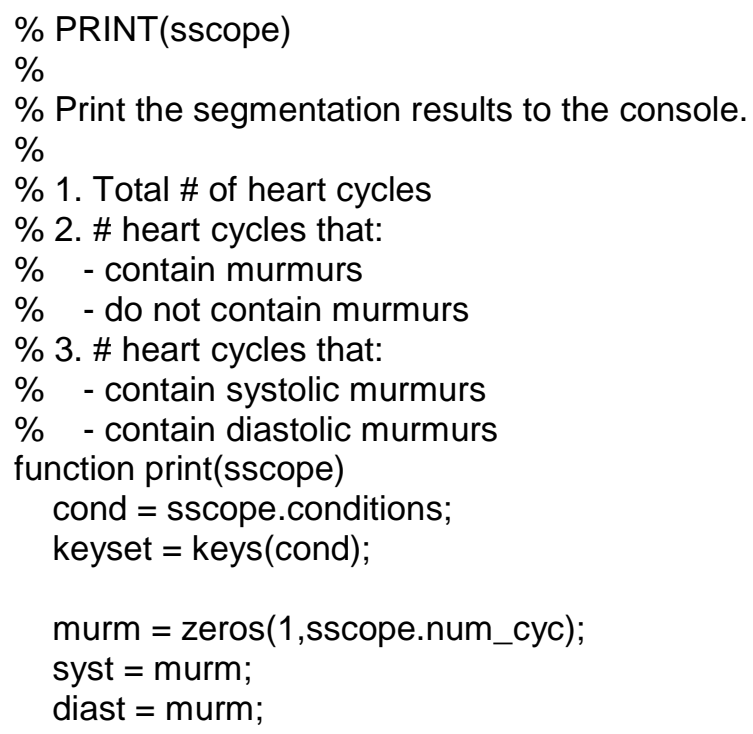




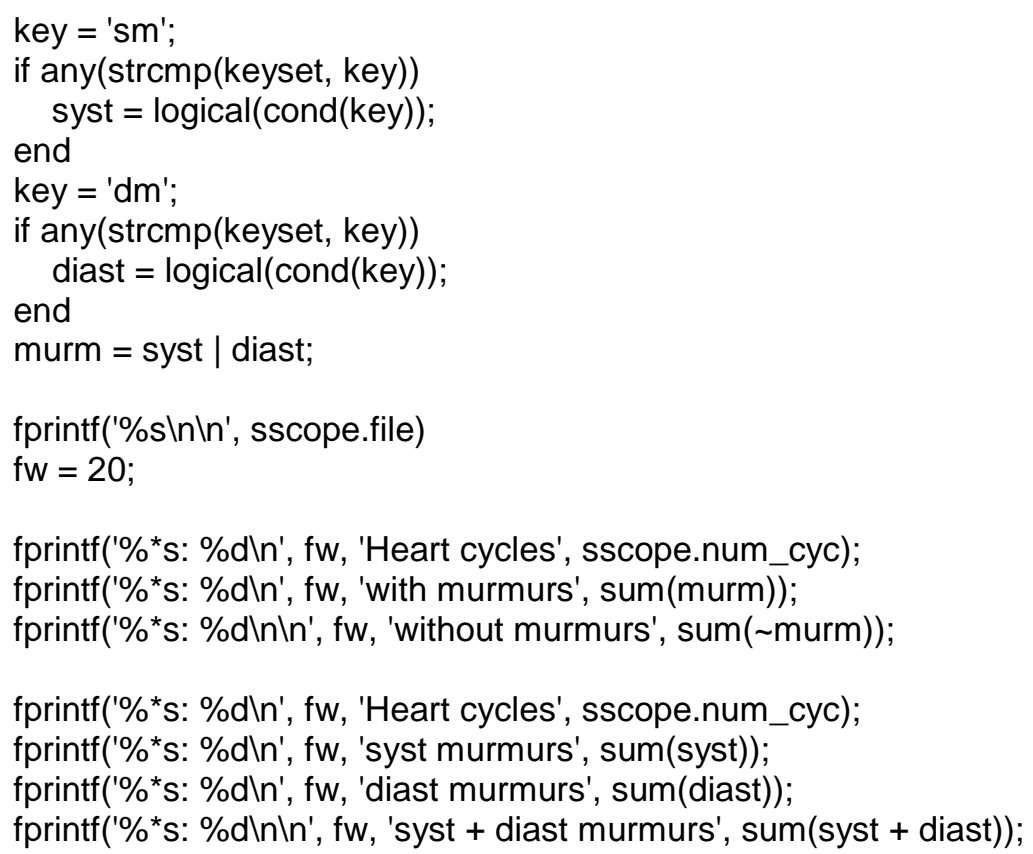

\section{C.2.6 simpl_segment.m}

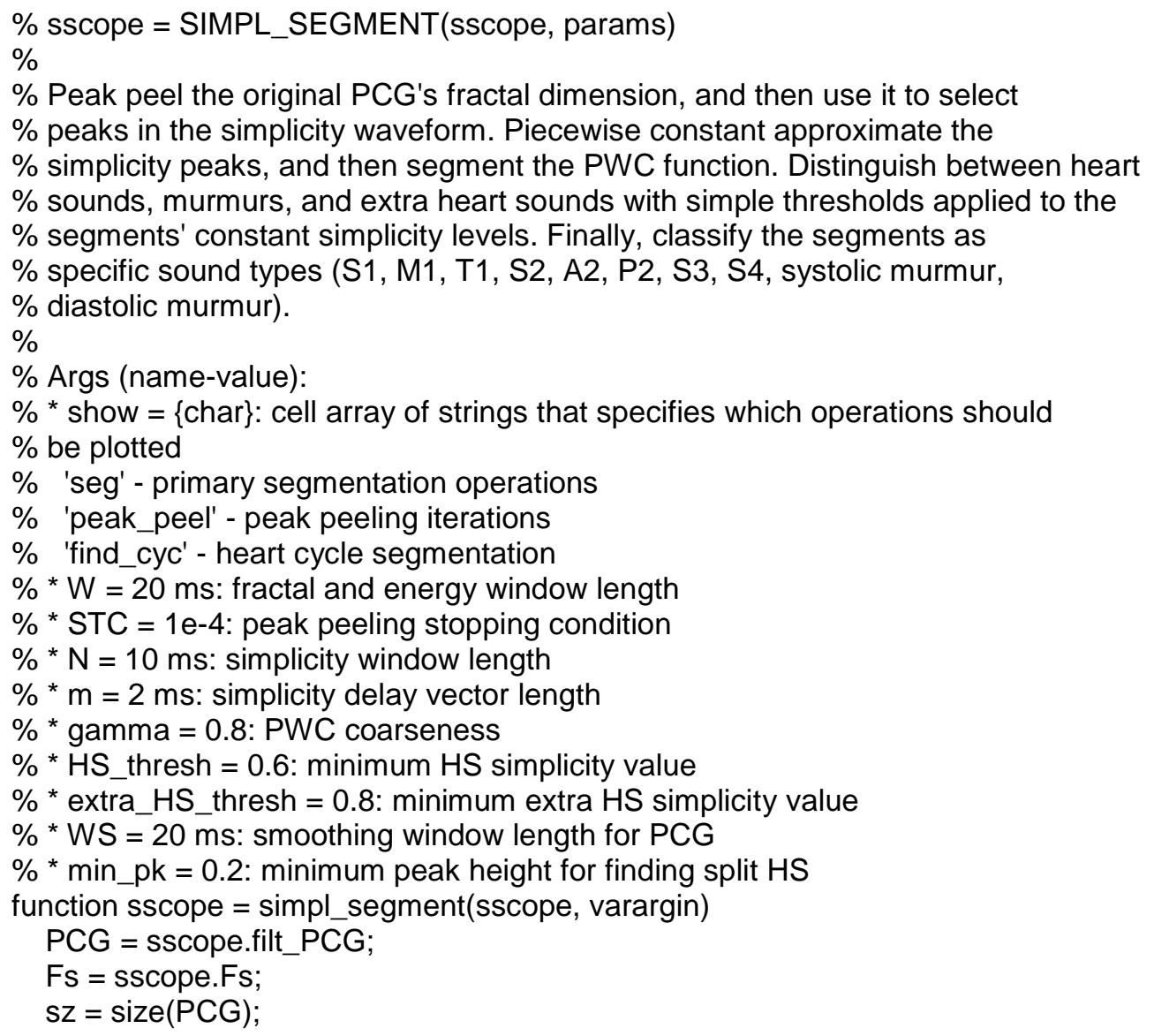




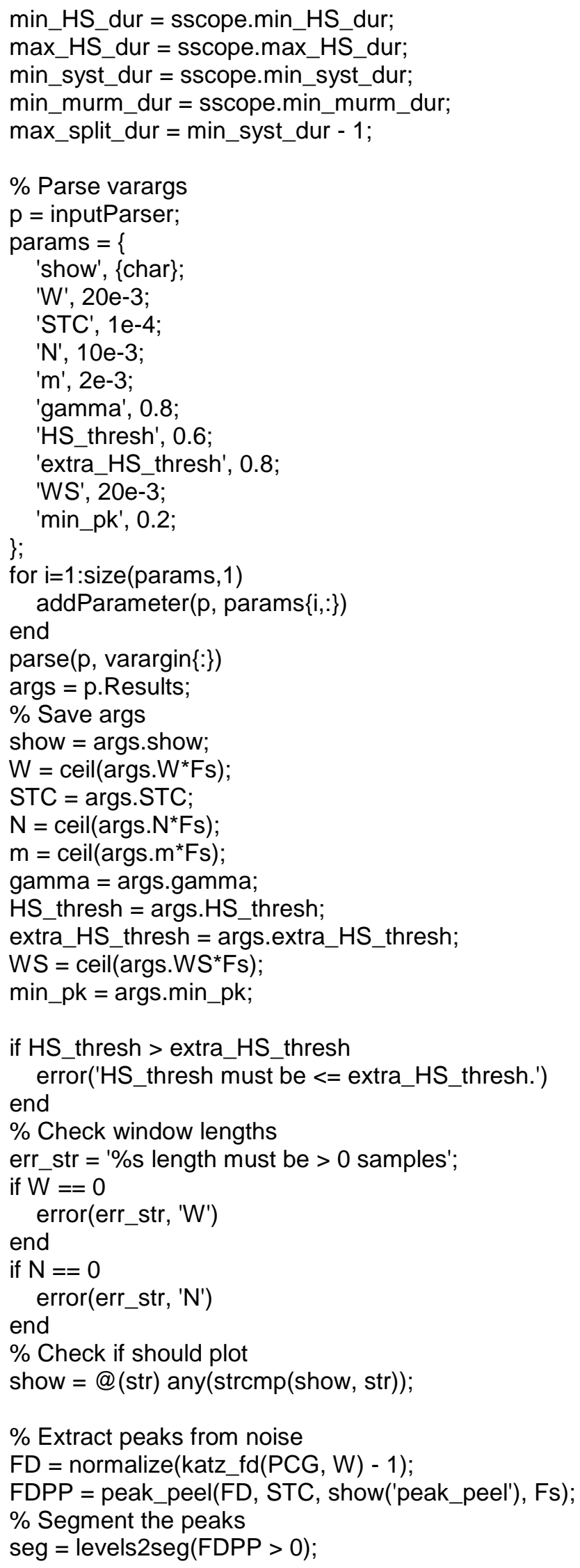




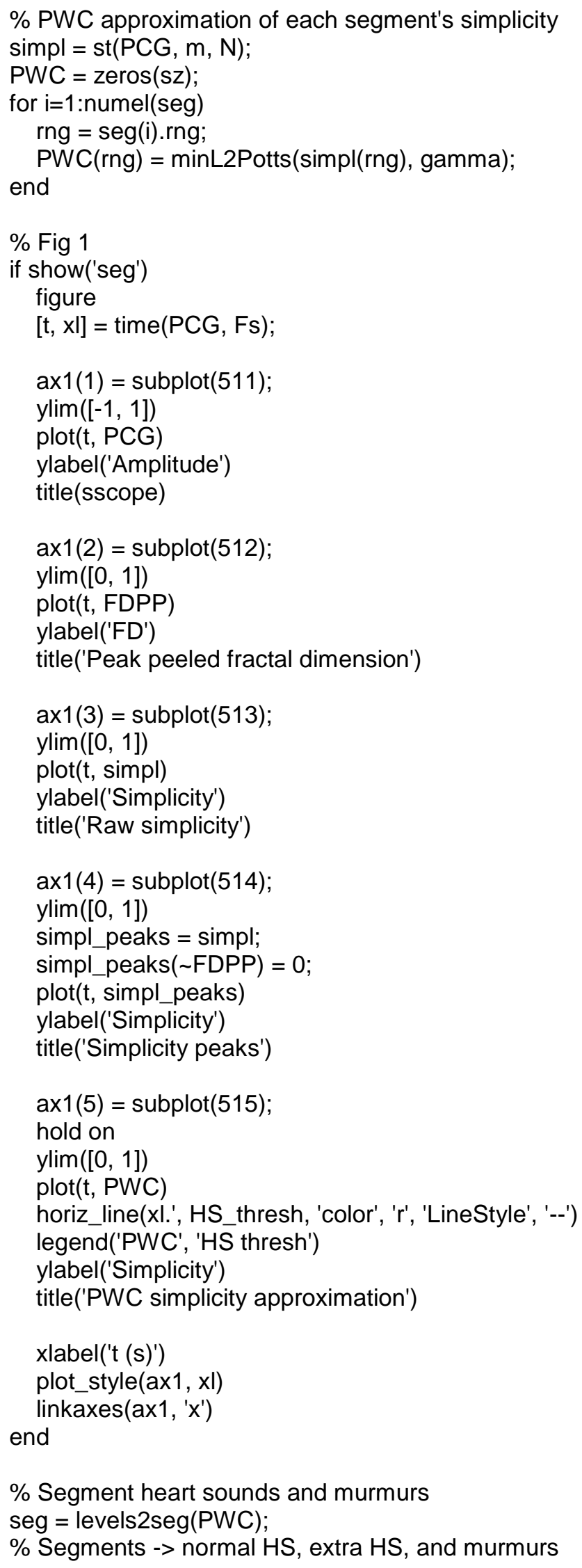




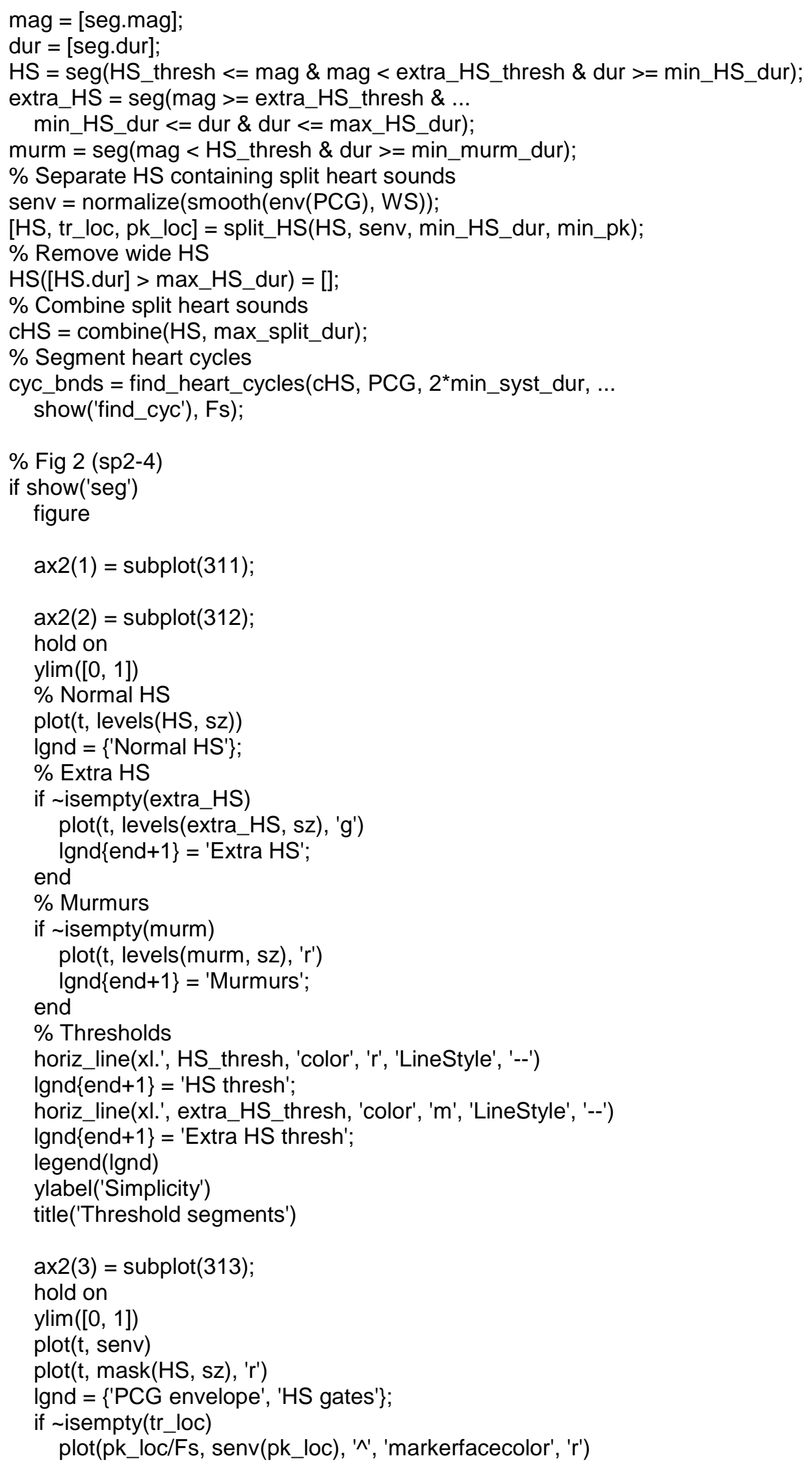




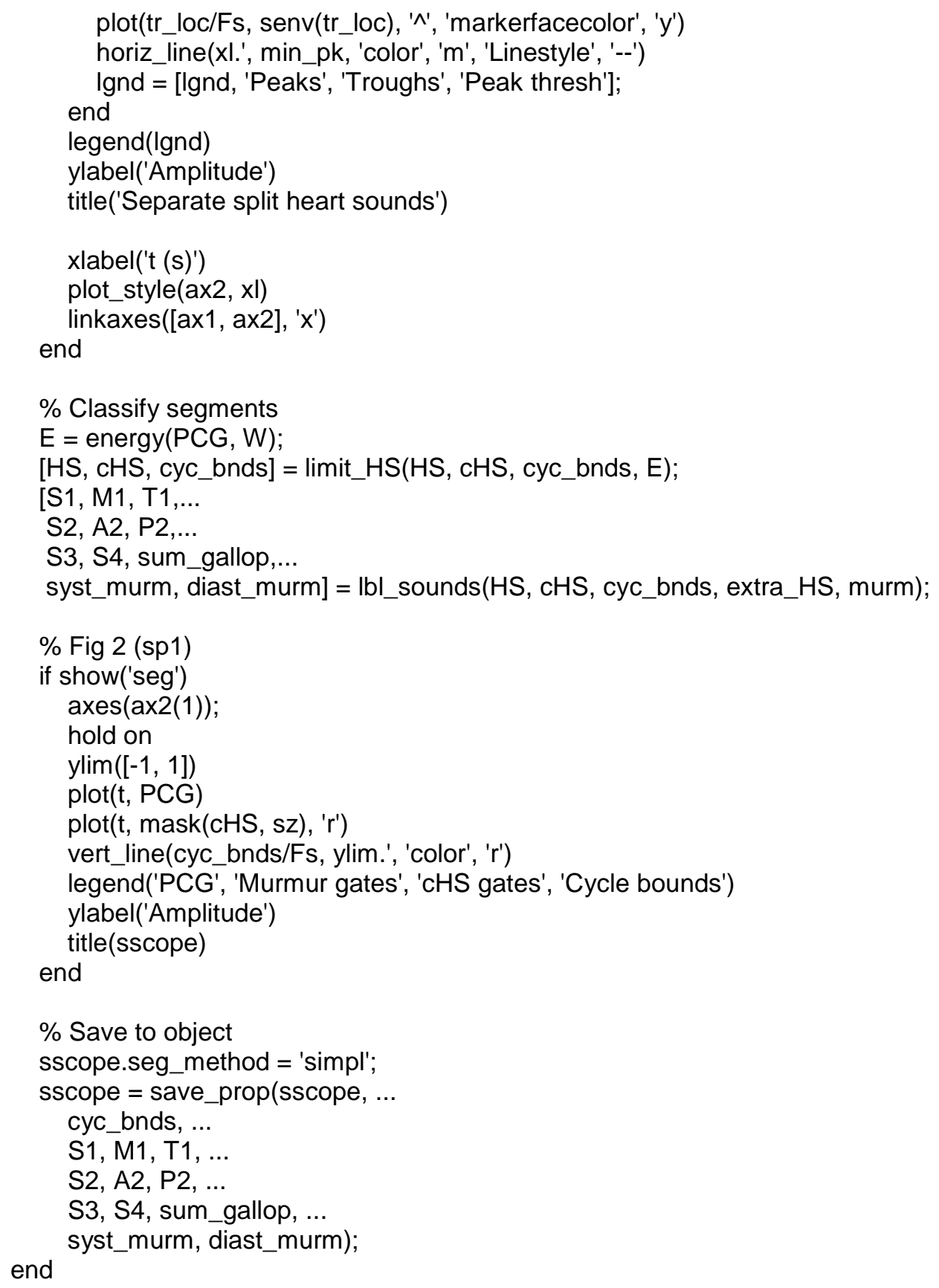

\section{C.2.7 stethoscope.m}

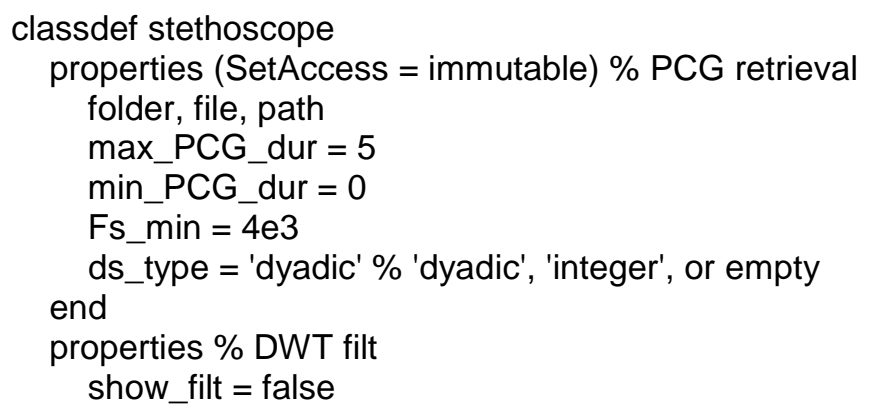




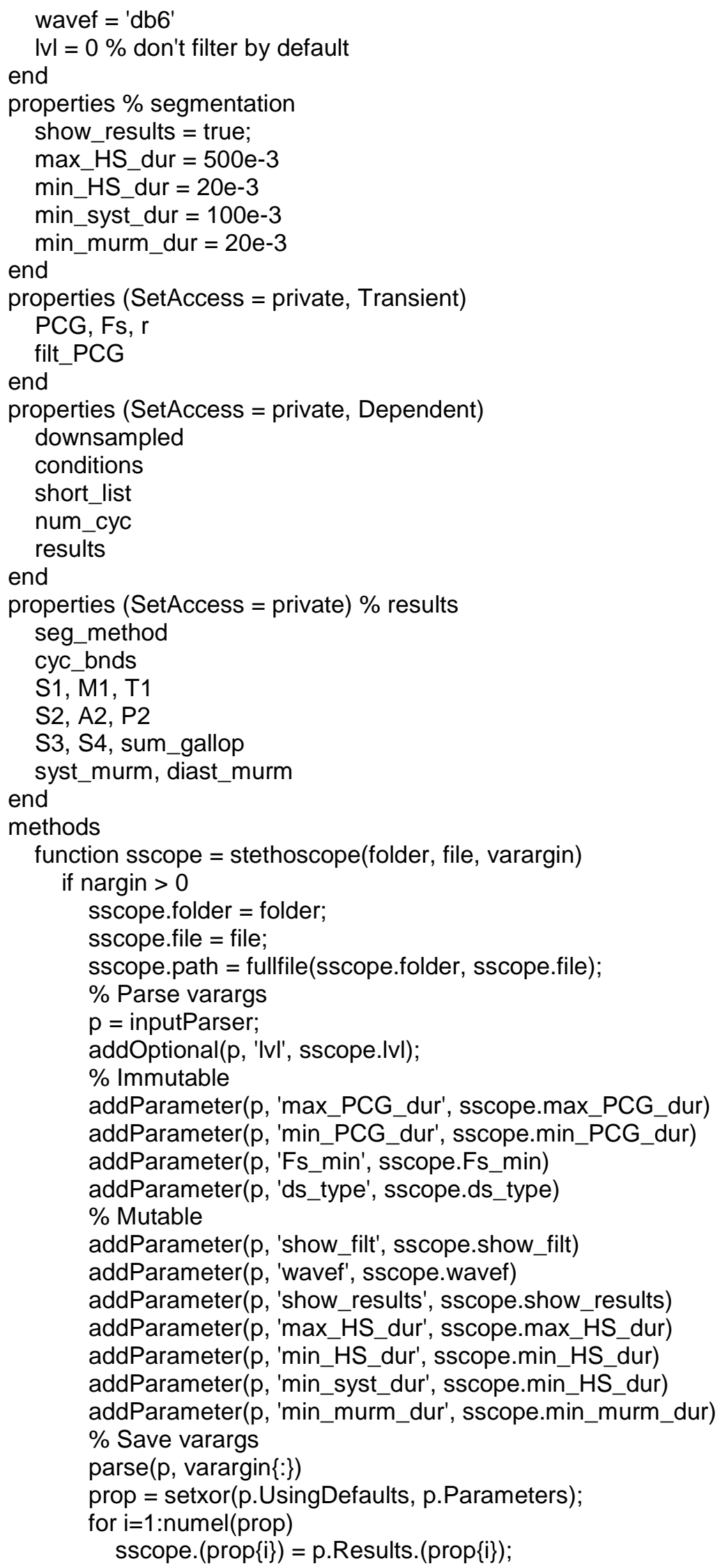




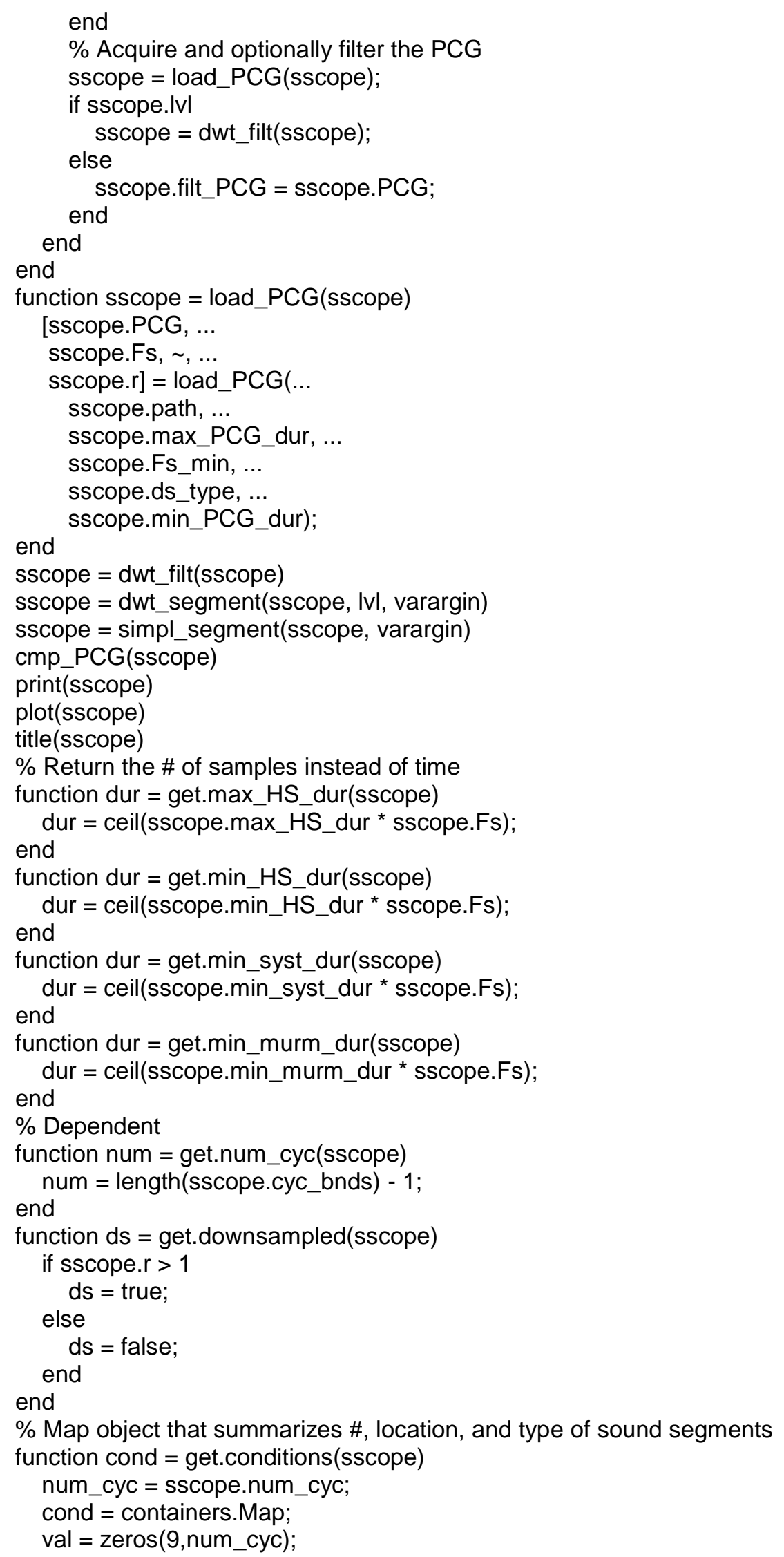




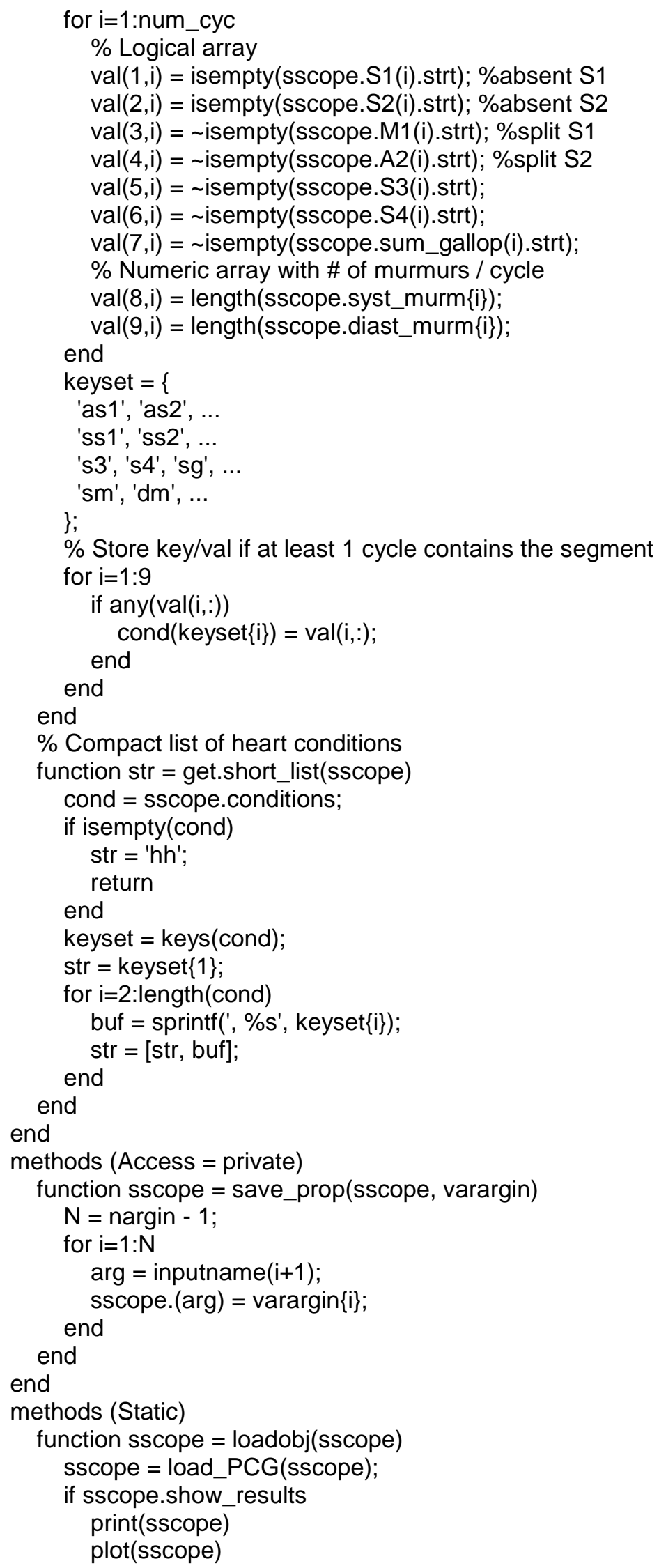




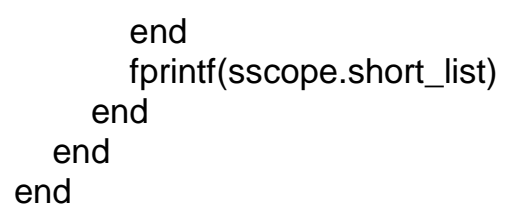

\section{C.2.8 title.m}

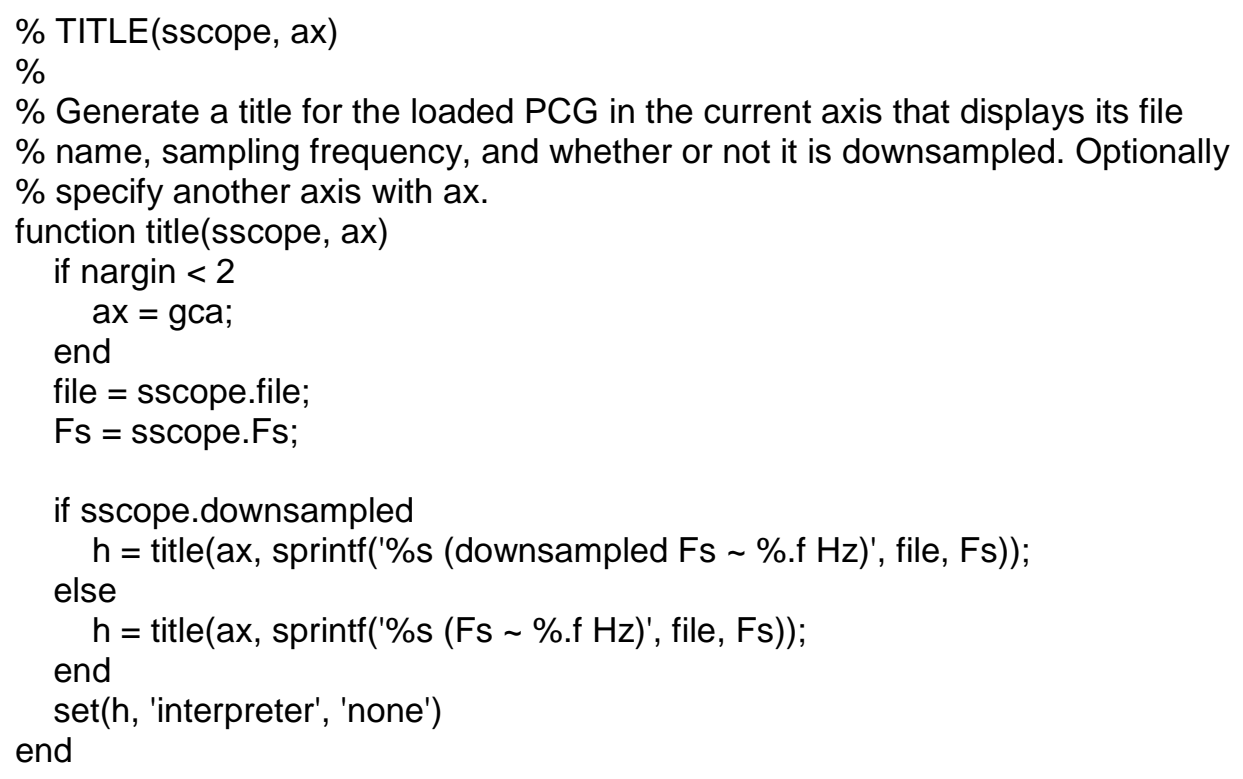

